\title{
Study of the Inclusive Beauty Production at CMS and \\ Construction and Commissioning of the CMS Pixel Barrel Detector
}

\author{
A dissertation submitted to \\ ETH ZURICH \\ for the degree of \\ DOCTOR OF SCIENCES \\ presented by \\ Lea Michaela CAminada \\ Dipl. Phys. ETH \\ born on December 30, 1981 \\ citizen of Zürich (ZH) und Vrin (GR)
}

accepted on the recommendation of

Prof. Dr. Felicitas Pauss (examiner),

Prof. Dr. Ralph Eichler (co-examiner) and

Prof. Dr. Roland Horisberger (co-examiner) 



\begin{abstract}
Beauty quarks are produced with a large cross section at a yet unreached center-of-mass energy at the Large Hadron Collider (LHC), enabling precision measurements to improve our understanding of heavy flavor physics. Within this thesis a study of the inclusive $b$-quark production at the CMS experiment is presented. As a result an analysis strategy is proposed based on the reconstruction of muons produced in the semileptonic decay of $b$ quarks. The semileptonic decays are exploited in the kinematic range of muon transverse momentum $p_{T}>6 \mathrm{GeV}$ and muon pseudorapidity $-2.1<$ $\eta<2.1$.

The analysis is applied to data recorded by the CMS detector during the first months of high-energy collision data-taking in April and May 2010, corresponding to an integrated luminosity of $\mathcal{L}=8.1 \mathrm{nb}^{-1}$. For the first time, the total $b$-quark production cross-section has been measured at a center-of-mass energy of $\sqrt{s}=7 \mathrm{TeV}$. The differential $b$-quark production cross-section as a function of muon transverse momentum and pseudorapidity is determined and compared to leading-order and next-to-leading-order QCD predictions.
\end{abstract}

The second part of the thesis focusses on the construction and commissioning of the CMS pixel barrel detector, the central part of the CMS detector with about 48 million readout channels. The CMS pixel detector allows to measure secondary vertices with high precision and thus plays a key role in the analysis of events with $b$-quarks.

The integration of the CMS pixel barrel detector has been accomplished within about two years before it was installed into the CMS detector in Summer 2008. The large effort in commissioning and calibration resulted in the successful and stable operation of the CMS pixel detector during cosmic and proton-proton collision data taking. 



\section{Zusammenfassung}

Beauty Quarks werden am Large Hadron Collider (LHC) mit einem grossen Wirkungsquerschnitt und bei einer zuvor unerreichten Schwerpunktsenergie erzeugt. Dadurch werden Präzisionsmessungen möglich, die unser Wissen über die Physik der schweren Quarks erweitern werden.

Diese Doktorarbeit befasst sich mit der Untersuchung der inklusiven $b$ Quark Produktion am CMS Experiment. Die vorgestellte Analyse basiert auf der Rekonstruktion von Muonen aus dem semileptonischen Zerfall von $b$-Quarks. Der kinematische Bereich der Muonen ist definiert durch den Transversalimpuls $p_{T}>6 \mathrm{GeV}$ und die Pseudorapidität $-2.1<\eta<2.1$.

Die Analyse wird anhand von Daten durchgeführt, die vom CMS Detektor im April und Mai 2010 während der ersten Datennahme von hochenergetischen Proton-Proton Kollisionen aufgezeichnet wurden. Der Datensatz entspricht einer integrierten Luminosität von $\mathcal{L}=8.1 \mathrm{nb}^{-1}$. Der totale inklusive Wirkungsquerschnitt der $b$-Quark Produktion bei einer Kollisionsenergie von $\sqrt{s}=7 \mathrm{TeV}$ wird zum ersten Mal gemessen. Der differentielle Wirkungsquerschnitt in Abhängigkeit des Transversalimpulses und der Pseudorapidität der Muonen wird ebenfalls bestimmt und mit leading-order und next-to-leading-oder QCD Vorhersagen verglichen.

Im zweiten Teil dieser Arbeit wird der Bau und die Inbetriebnahme des CMS Pixel Barrel Detektors vorgestellt. Der CMS Pixel Barrel Detektor bildet den innersten Teil des CMS Detektors mit etwa 48 Millionen Auslesekanälen. Der Pixel Detektor ermöglicht eine äusserst genaue Messung von sekundären Vertices aus dem Zerfall von schweren Teilchen und ist daher von entscheidender Bedeutung in der Analyse von Ereignissen mit $b$-Quarks.

Die Fertigstellung des CMS Pixel Barrel Detektors nahm etwa zwei Jahre in Anspruch und der Detektor konnte im Sommer 2008 in den CMS Detektor installiert werden. Die Anstrengungen, die unternommen wurden um den Detektor in Betrieb zu nehmen und zu kalibrieren, bilden die Grundlage für die zuverlässige und äusserst erfolgreiche Datennahme bei der Aufzeichnung von Spuren von kosmischen Teilchen und Teilchen, die in Proton-Proton Kollisionen erzeugt wurden. 



\section{Contents}

1 Introduction 11

2 The CMS Experiment at the LHC 15

2.1 The Large Hadron Collider . . . . . . . . . . . . . . . . . . . 15

2.2 The CMS Detector . . . . . . . . . . . . . . . . . 16

2.2 .1 Coordinate Conventions . . . . . . . . . . . . . . . 18

2.2 .2 Solenoid . . . . . . . . . . . . . . . . . . . 18

2.2 .3 Tracking Detectors . . . . . . . . . . . . . . . . . . 18

2.2 .4 Track Reconstruction . . . . . . . . . . . . . . . . . . 19

2.2.5 Electromagnetic Calorimeter . . . . . . . . . . . . . . . 21

2.2 .6 Hadronic Calorimeter . . . . . . . . . . . . . . . . . . 23

2.2 .7 Muon System . . . . . . . . . . . . . . . . . . . . . . 24

2.2 .8 Muon Reconstruction . . . . . . . . . . . . . . . . 26

2.2 .9 Trigger System . . . . . . . . . . . . . . . . . . 26

I Study of the Inclusive $b$ Quark Production at CMS 29

3 Heavy Flavor Physics 31

3.1 Quantum Chromodynamics ........................ 31

3.2 Hadronic Collisions . . . . . . . . . . . . . . . . . . . . . . . 32

3.2 .1 Event Kinematics . . . . . . . . . . . . . . . . . . . . . . 33

3.2 .2 Factorization . . . . . . . . . . . . . . . 33

3.2.3 Evolution of Parton Distribution Functions . . . . . . . . . . . . 35

3.3 Heavy Quark Production . . . . . . . . . . . . . . . . . 36

3.4 The Fragmentation of Heavy Quarks . . . . . . . . . . . . . . . . . . 37

3.5 Semileptonic Decays of Heavy Quarks . . . . . . . . . . . . . . . . . . 39

3.6 Monte Carlo Event Generators . . . . . . . . . . . . . . . . . . . . . 41

4 Study of the Inclusive Beauty Production 45

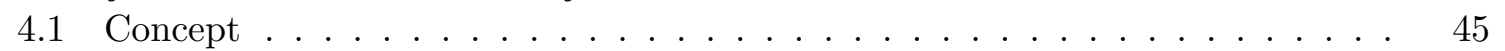

4.2 Event Simulation . . . . . . . . . . . . . . . . . . . . . . . 46

4.3 Trigger . . . . . . . . . . . . . . . . . . . . . . . . . 48

4.4 Jet Reconstruction . . . . . . . . . . . . . . . . . . . . . . . . . . . . . . . . . . . . . . . . . . . . .

4.5 Event Selection . . . . . . . . . . . . . . . . . . 54

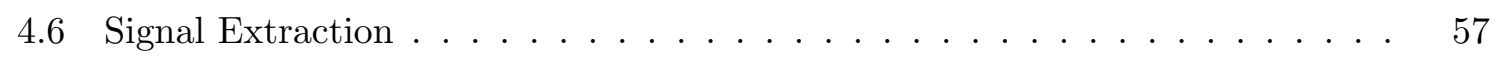

4.6 .1 Fitting Procedure . . . . . . . . . . . . 57 
4.6 .2 Performance of the Fit . . . . . . . . . . . . . . . 59

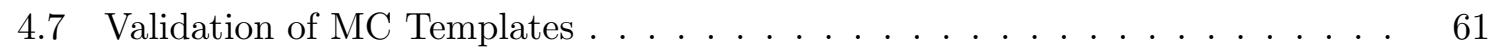

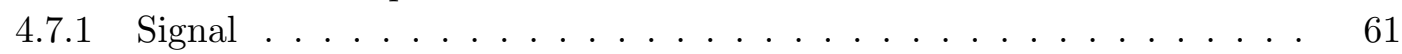

4.7 .2 Background . . . . . . . . . . . . . . . . . . . . . 62

4.8 Cross Section Measurement . . . . . . . . . . . . . . . . . 65

4.8 .1 Inclusive Cross Section . . . . . . . . . . . . . . . . 65

4.8.2 Differential Cross Section . . . . . . . . . . . . . 65

4.9 Systematic Uncertainties . . . . . . . . . . . . . . . . . . . . . . . . . . . . . . . . . . . . . 67

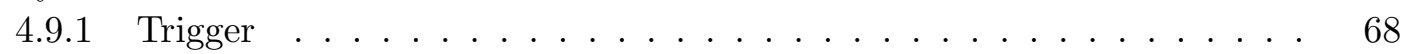

4.9.2 Tracking Efficiency and Misalignment . . . . . . . . . . . 68

4.9.3 Background Composition . . . . . . . . . . . . . . . 68

4.9 .4 Fragmentation and Decay . . . . . . . . . . . . . . . 69

4.9.5 Production Mechanism . . . . . . . . . . . . . . . 70

4.9.6 Description of the Underlying Event . . . . . . . . . . . . . . 71

4.9 .7 Monte Carlo Statistics . . . . . . . . . . . . . . . . . . . . . . . . . . 71

4.9 .8 Luminosity . . . . . . . . . . . . . . . . . . . . . . . . . . . . . . . . . . . . .

4.9 .9 Summary . . . . . . . . . . . . . . . . . . . . . . . . . . . . 72

4.10 Results . . . . . . . . . . . . . . . . . . 72

$5 \quad$ Results of First Collisions at $\sqrt{s}=900 \mathrm{GeV}$ and $\sqrt{s}=2.36 \mathrm{TeV} \quad \mathbf{7 7}$

5.1 Event Selection . . . . . . . . . . . . . . . . . . . . 77

5.2 Event Simulation . . . . . . . . . . . . . . . . . . . . . . . 78

5.3 Muon Distributions . . . . . . . . . . . . . . . . . . . . . . . 78

5.4 Track Distributions . . . . . . . . . . . . . . . . . . . 80

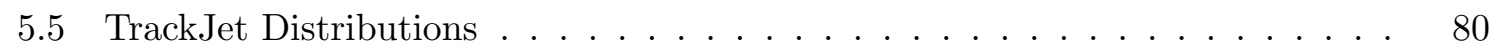

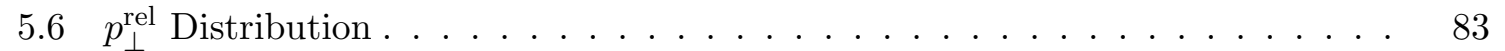

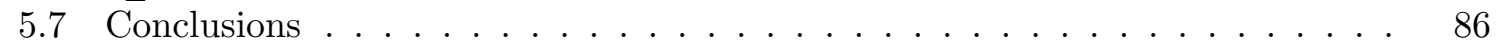

$6 \quad$ Preliminary Results of First Collisions at $\sqrt{s}=7 \mathrm{TeV} \quad \mathbf{8 7}$

6.1 Event Simulation . . . . . . . . . . . . . . . . . 87

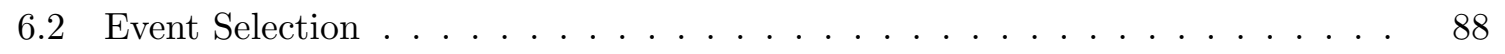

6.2.1 Run Selection . . . . . . . . . . . . . . . 88

6.2 .2 Trigger Selection . . . . . . . . . . . . . . . . . . 89

6.2 .3 Offline Selection . . . . . . . . . . . . . . . . . . 89

6.3 Signal Extraction . . . . . . . . . . . . . . . . . . . . 90

6.3.1 Data-driven Determination of Light Quark Background . . . . . . . . 90

6.3.2 Data-driven Validation of $p_{\perp}^{\text {rel }}$ Templates in Signal Events . . . . . . . 92

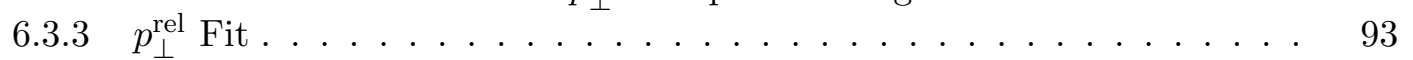

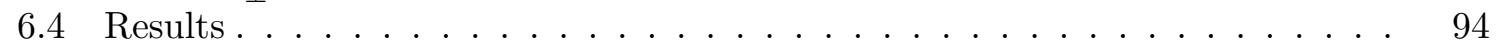

6.5 Systematic Uncertainties . . . . . . . . . . . . . . . . . . . . . 99

6.6 Conclusions . . . . . . . . . . . . . . . . . . . . 100

\section{Construction and Commissioning of the CMS Pixel Barrel Detector 103}

7 The CMS Pixel Barrel Detector $\quad 105$

7.1 Design of the CMS Pixel Barrel Detector . . . . . . . . . . . . 105 
7.2 Detector Modules . . . . . . . . . . . . . . . . . . 106

7.2 .1 Sensor . . . . . . . . . . . . . . . . . . 106

7.2 .2 Readout Chip . . . . . . . . . . . . . . . . . . . . . 107

7.2 .3 Token Bit Manager . . . . . . . . . . . . . . . . . . . . . 108

7.3 Readout and Control System _. . . . . . . . . . . . . . . . . 110

7.3 .1 Analog Chain . . . . . . . . . . . . . . . . . 111

7.3 .2 Front End Driver . . . . . . . . . . . . . . . . . . . . 112

7.3 .3 Supply Tube . . . . . . . . . . . . . . . . . . . . . . . 112

7.3.4 Communication and Control Unit . . . . . . . . . . . . . . . . . 112

7.3.5 Front End Controller . . . . . . . . . . . . . . . . . . . . . 113

8 Construction and Commissioning of the CMS Pixel Barrel Detector 115

8.1 Low Level Hardware Testing Procedure . . . . . . . . . . . . . . . . . . . . . 115

8.1 .1 Test Setup . . . . . . . . . . . . . . . . 116

8.1 .2 Software Architecture . . . . . . . . . . . . . . . . 116

8.1 .3 Testing Sequence . . . . . . . . . . . . . . . . . . 116

8.2 Performance Tests and Calibrations _ . . . . . . . . . . . . . . . . 121

8.3 Construction . . . . . . . . . . . . . . . . . . . . 124

8.3 .1 Module Mounting . . . . . . . . . . . . . . . . . . . . . . . . 124

8.3 .2 Supply Tube Assembly . . . . . . . . . . . . . . . . . . . . . . . . . . . . . . . . . 125

8.3.3 Integration of the Complete System . . . . . . . . . . . . . 128

8.4 Installation into CMS . . . . . . . . . . . . . . . . . . . 129

8.5 Commissioning and Performance . . . . . . . . . . . . . . . . 130

8.5.1 Performance of the Optical Links . . . . . . . . . . . . . . . . 131

8.5.2 Detector Module Functionality . . . . . . . . . . . . . . . . . . 133

8.5.3 Results of the Detector Calibration . . . . . . . . . . . . . . 133

8.5.4 Results of the Cosmic Run . . . . . . . . . . . . . . . . . 133

8.6 Summary . . . . . . . . . . . . . . . . . . 135

9 Conclusion and Outlook $\quad 137$

A Maximum Likelihood Fits 139

A.1 Bins in Muon Transverse Momentum . . . . . . . . . . . . . . . . . . . 140

A.1.1 Fit Result . . . . . . . . . . . . . . . . . . . . . . . . . 140

A.1.2 Fit Deviation in the Approximate Method . . . . . . . . . . . . . . . 141

A.1.3 Pull Distributions of the Approximate Method . . . . . . . . . . . . . 142

A.1.4 Fit Deviation in the Full Treatment . . . . . . . . . . . . . . . . . . 143

A.1.5 Pull Distributions of the Full Treatment . . . . . . . . . . . . . . . . . 144

A.2 Bins in Muon Pseudorapidity . . . . . . . . . . . . . . . . 145

A.2.1 Fit Result . . . . . . . . . . . . . . . . . . . . 145

A.2.2 Fit Deviation in the Approximate Method . . . . . . . . . . . . . 146

A.2.3 Pull Distributions of the Approximate Method . . . . . . . . . . . . 147

A.2.4 Fit Deviation in the Full Treatment . . . . . . . . . . . . . . 148

A.2.5 Pull Distributions of the Full Treatment . . . . . . . . . . . . . . 149

B Systematic Uncertainties $\quad 151$

B.1 Limited MC Statistics . . . . . . . . . . . . . . . . . . . . 152 
C Preliminary Results of First Collisions at $\sqrt{s}=7 \mathrm{TeV} \quad 153$

C.1 $p_{\perp}^{\text {rel }}$ Distribution in Bins of Muon Transverse Momentum . . . . . . . . . . . . 154

C.2 $p_{\perp}^{\text {rel }}$ Distribution in Bins of Muon Pseudorapidity . . . . . . . . . . . . . 155 


\section{Chapter 1}

\section{Introduction}

The Standard Model (SM) of elementary particle physics provides a remarkably accurate picture of the fundamental structure of matter. Since its introduction in the early 1970's by Sheldon Glashow [1], Steven Weinberg [2] and Abdus Salam [3], it has successfully explained a wide range of experimental results and precisely predicted a large variety of phenomena from the elementary world to the universe.

The SM is the theory describing the fundamental particles and their interactions based on relativistic quantum field theory. It consists of 12 spin- $\frac{1}{2}$ fermions and 4 spin- 1 gauge bosons which act as carrier of the fundamental forces. Three of the four fundamental forces, namely electromagnetism, weak interaction and strong interaction, are included in the SM. The gravitational force which is dominant at macroscopic distances is omitted since its effects are small on microscopic scales. The 12 fermions are divided into 6 quarks $(u, d, s, c, b, t)$ and 6 leptons $\left(e, \mu, \tau, \nu_{e}, \nu_{\mu}, \nu_{\tau}\right)$, all of which possess charge conjugate states, called anti-particles. Moreover, the quarks and leptons are classified into three generations with very different mass scales ranging in case of the quarks from a few $\mathrm{MeV}$ for up and down quarks to about $171 \mathrm{GeV}$ for the top quark.

All quarks and leptons are subject to the weak force, while only quarks and charged leptons $(e, \mu, \tau)$ undergo electromagnetic interactions. A major achievement of the SM is the unification of the electromagnetic and the weak force into an electroweak force embedded in the theory of Quantum Electrodynamics (QED). Therein the photon is the gauge boson of the electromagnetism and the $W$ - and $Z$-bosons are the mediators of the weak interaction. The strong force is responsible for the interaction between color charged quarks and gluons where the latter are the force carriers in the theory of Quantum Chromodynamics (QCD).

It should be emphasized that seven $\left(c, b, t, \nu_{\tau}, W / Z\right.$, gluon) out of the 16 particles have been predicted by the SM before they have been observed experimentally. The SM is completed by the introduction of an additional particle called the Higgs boson. The Higgs boson plays an important role in the SM as it provides an explanation for the masses of the elementary particles and gives rise to the phenomenon of electroweak symmetry breaking. Despite the large effort, the experimental verification of the existence of the Higgs boson has not been crowned with success so far.

The search for the elusive Higgs particle has been one of the main motivations for the construction of the Large Hadron Collider (LHC) at CERN. The LHC is a proton-proton collider which has been designed to operate at a center-of-mass energy of $\sqrt{s}=14 \mathrm{TeV}$. LHC operation started on March 30, 2010 and the first collisions happened at a center-of-mass 
energy of $\sqrt{s}=7 \mathrm{TeV}$. It is foreseen to collect a significant amount of data at this lower energy before the collision energy will be increased to the design value in 2013. The energy of $\sqrt{s}=14 \mathrm{TeV}$ is high enough to produce the Higgs boson and access the yet unexplored mass range up to about $1 \mathrm{TeV}$. The experiments at the LHC are supposed to finally answer one of the most urgent questions of modern particle physics and to either detect the Higgs boson and confirm the SM prediction or to exclude its existence and falsify this part of the SM.

In spite of the outstanding achievement of the SM in describing various aspects of the physics within its domain, it is nevertheless an incomplete theory. First of all, the theoretical framework is known to be no longer self-consistent when approaching energies of a few $\mathrm{TeV}$. Furthermore, the SM leaves many fundamental questions unanswered, concerning for instance the nature of dark matter or the origin of the asymmetry between matter and antimatter in our universe. In an endeavor to provide explanations for these questions, many promising theories extending the SM have been developed. Supersymmetric theories or theories with extra-dimensions are the most prominent examples. Many of these theories predict physics effects which should be accessible at the energy scale available at the LHC. Hopes are high for discovering new phenomena at the LHC which would challenge the SM and possibly give crucial information about physics beyond the SM.

Owing to the need of good statistics high quality data for the search of the Higgs boson, the potential discovery will take its time. The short term goal of the CMS physics program is therefore the experimental confirmation of the SM at the higher energy scale the LHC is capable of providing.

Within the thesis presented here the production of beauty quarks at the LHC is studied at the CMS experiment. Due to the large b-quark production cross-section at the LHC, high statistics data samples are available soon after the LHC startup which renders the CMS experiment an excellent facility for the study of heavy flavor physics.

The production of heavy quarks is expected to be accurately described by perturbative QCD. The investigation of events containing beauty quarks is interesting as it allows to probe the predictions of the theory of strong interactions at the energy scale provided by the LHC. Furthermore, the production of $b$ quarks is a major source of background for many searches to be performed at the LHC and therefore has to be well understood.

A measurement of the beauty quark production cross-section based on the semileptonic decay of $b$ quarks into muons is performed in this thesis. Because of the large mass of the $b$ quark, muons from semileptonic $b$-decays have larger transverse momenta with respect to the quark direction than muons from the decay of lighter quarks. In the experiment, the quark direction is approximated by the axis of the fragmentation jet and the transverse momentum of the muon relative to that axis $\left(p_{\perp}^{\mathrm{rel}}\right)$ is measured in data. The contribution of $b$-events to the measured distribution is determined by performing a fit based on simulated template distributions for signal and background events.

The method of discriminating $b$-events from background events by means of the $p_{\perp}^{\text {rel }}$ variable has been used for the first time by the UA1 experiment at the CERN SPS collider [4]. Since then it has become a well-established technique for the identification of beauty quarks: the $p_{\perp}^{\text {rel }}$ method has been further applied for measuring the $b$-quark production cross-section at electron-positron [5, 6], electron-proton [7] and hadron-hadron colliders [8, 9].

Measurements of the $b$-quark production cross-section are available from collider experiments at different center-of-mass energies. A lot of progress has been made in understanding the $b$-quark production process [10], so that the measurements are meanwhile in reasonable 
agreement with the theoretical predictions in most regions of the phase space. Nonetheless, there is a great interest in verifying these results at the higher center-of-mass energy of the LHC, since the theoretical uncertainties are still sizeable. A first measurement of the $b$-quark production cross-section using the very early data from the LHC is presented within this work.

Even though the CMS detector is primarily designed for high transverse momentum physics, it is very well suited for heavy flavor physics thanks to the muon system with the potential to identify low transverse momentum muons and the excellent tracking detectors. In particular, CMS features a novel three-layer silicon pixel detector which allows for a precise and efficient reconstruction of secondary vertices from heavy flavor decays.

The barrel part of the CMS pixel detector was developed, designed and built at PSI in cooperation with ETH Zürich and the University of Zürich. In the framework of this thesis important contributions to the construction and commissioning of the pixel detector were made. This includes the assembly of the complete system together with the control and data acquisition system at PSI, the construction of the final system and the development of software algorithms needed for testing and calibration. The CMS pixel detector was installed into the CMS detector in summer 2008 and after a period of commissioning and calibration successfully operated during both cosmic and collision data taking.

The thesis is organized as follows: In the first chapter an overview of the experimental facility at the LHC and the CMS detector is presented. The theoretical framework important for the physics analysis described herein is introduced in chapter 3. This includes a review of the main ideas of Quantum Chromodynamics and its application to hadronic collisions, an introduction to the physics of heavy quarks and an overview of the Monte Carlo event generators used in the analysis. Chapter 4 is devoted to the study of the inclusive $b$-quark production cross-section at CMS. The prospects for the measurement were determined based on simulated data. Furthermore, a detailed study of the main systematic effects of the measurement is accomplished. In December 2009, a first commissioning run producing proton-proton collisions at center-of-mass energies of $\sqrt{s}=900 \mathrm{GeV}$ and $\sqrt{s}=2.36 \mathrm{TeV}$ took place at the LHC. The data collected during this run was analyzed within this work and the results relevant for the study of the beauty production are summarized in chapter 5 . In chapter 6 the preliminary results of the measurement of the inclusive $b$-quark production cross-section at a center-ofmass energy of $\sqrt{s}=7 \mathrm{TeV}$ are presented. The measurement is based on the collision data recorded by the CMS experiment during the first months of high-energy collision data-taking in April and May 2010.

The second part of the thesis concentrates on the hardware related work performed within the CMS pixel detector group at PSI. In chapter 7 the main aspects of the design and the functionality of the CMS pixel barrel detector are addressed, while chapter 8 focusses on how this work contributed to the construction and commissioning of the detector.

Throughout this thesis, natural units are used in which $c=\hbar=1$. 



\section{Chapter 2}

\section{The CMS Experiment at the LHC}

The Large Hadron Collider (LHC) [11] is designed to collide proton beams at a center-of-mass energy of $\sqrt{s}=14 \mathrm{TeV}$ and a nominal instantaneous luminosity of $\mathcal{L}=10^{34} \mathrm{~cm}^{-2} \mathrm{~s}^{-1}$. This represents a seven-fold increase in energy and a hundred-fold increase in integrated luminosity over the previous hadron collider experiments. The beam energy and the design luminosity have been chosen in order to study physics at the TeV energy scale. The main motivation of the LHC is to reveal the nature of electroweak symmetry breaking and to investigate potential manifestations of new physics phenomena beyond the SM.

The unprecedented high center-of-mass energy and luminosity at the LHC lead to a number of substantial experimental challenges. In this chapter an introduction of the experimental facility is presented. An overview of the basic aspects of the LHC is followed by a detailed description of the CMS experiment and its main subdetectors.

\subsection{The Large Hadron Collider}

The LHC accelerator is located at CERN near Geneva in the already existing LEP tunnel which has a length of $26.7 \mathrm{~km}$. Four detectors are installed in the experimental caverns around the collision points: two general purpose experiments, ATLAS [12] and CMS [13, 14], the LHCb [15] experiment dedicated to $B$ Physics and the ALICE [16] experiment where the physics of heavy ion collisions is investigated.

To supply the LHC with pre-accelerated protons the existing CERN facilities have been upgraded. A schematic view of the LHC accelerator with the injection chain is shown in figure 2.1. The protons coming from the Super Proton Synchrotron (SPS) with an energy of $450 \mathrm{GeV}$ are injected into the LHC, where they will be accelerated to an energy of $7 \mathrm{TeV}$ in bunches with a nominal number of $1.15 \cdot 10^{11}$ particles per bunch. Superconducting dipole magnets which provide a magnetic field of $8.3 \mathrm{~T}$ are needed to keep the protons on the orbit during the acceleration. The superconducting magnets are cooled using liquid helium at a temperature of $1.9 \mathrm{~K}$.

At the interaction point where the CMS experiment is located collisions happen every $25 \mathrm{~ns}$, corresponding to a bunch crossing frequency of $40 \mathrm{MHz}$. The total proton-proton cross section at $\sqrt{s}=14 \mathrm{TeV}$ is expected to be about $100 \mathrm{mb}$. Therefore, approximately $10^{9}$ inelastic events per second will be observed in the multi-purpose experiments at design luminosity.

In the first year of LHC running the proton-proton collisions happen at a lower center-ofmass energy of $\sqrt{s}=7 \mathrm{TeV}$ and at a lower luminosity than what is foreseen in the original 
design. A commissioning run with collisions at center-of-mass energies of $\sqrt{s}=900 \mathrm{GeV}$ and $2.36 \mathrm{TeV}$ took place in November and December 2009. The LHC operation at $\sqrt{s}=7 \mathrm{TeV}$ started on March 30, 2010.

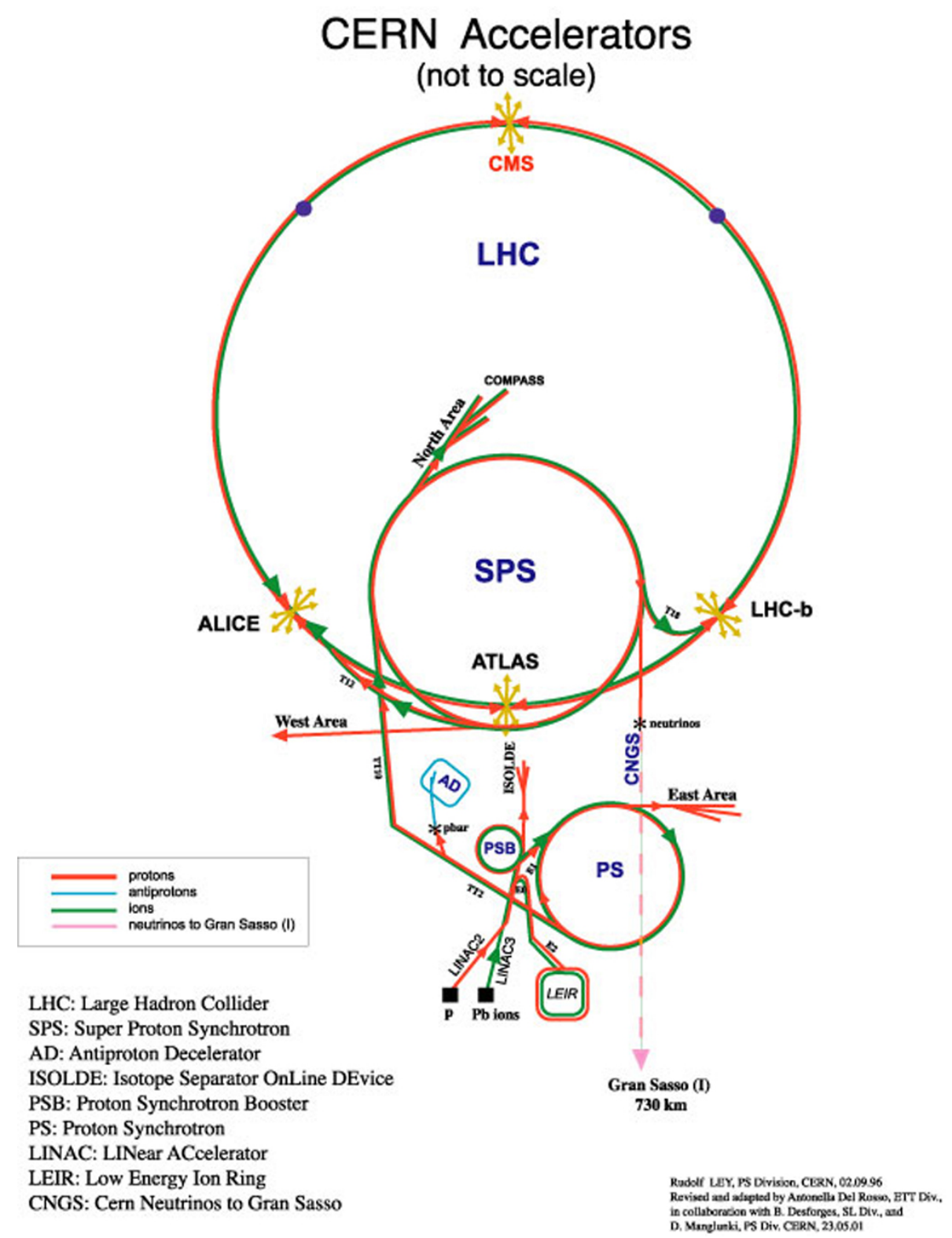

Figure 2.1: The CERN accelerator complex. Schematic view of the LHC and the location of the four main experiments ATLAS, CMS, LHCb and ALICE.

\subsection{The CMS Detector}

The CMS detector is a general purpose detector installed $100 \mathrm{~m}$ underground at the LHC interaction point 5 (P5) near the village of Cessy in France. The design of the CMS detector 
is driven by the challenges of a physics experiment in the LHC environment. Many of the physics benchmark channels have a small cross section and the background from QCD jet production is overwhelmingly dominant. A high rejection power with an optimal efficiency for rare channels has to be achieved. The reconstruction of lepton signatures is essential for the extraction of rare processes and an excellent muon and electron identification and momentum resolution is desired. Moreover, a precise measurement of secondary vertices and impact parameters is necessary for an efficient identification of heavy flavor and $\tau$-lepton decays.

The short bunch crossing separation and the high event rate at the LHC impose further challenges to the design. At design luminosity, 23 inelastic interaction per bunch crossing will occur on average. This phenomenon is know as pile-up. The effect of pile-up can be reduced by using high-granularity detectors resulting in low occupancy. This requires a large number of detector channels and an excellent synchronization among them. In addition, a good time resolution is needed to discriminate the interaction under study from the interactions occurring in neighboring bunch crossings. Another difficulty arises from the large flux of particles near the interaction point which leads to high radiation levels and the need of radiation hard detectors and front-end electronics.

The CMS detector is divided into a silicon tracking system, an electromagnetic and a hadronic calorimeter and a muon system. A magnetic field of $3.8 \mathrm{~T}$ is provided by a superconducting solenoid magnet. The CMS detector is $22 \mathrm{~m}$ long, has a diameter of $15 \mathrm{~m}$ and an overall weight of $12.5 \mathrm{t}$. Figure 2.2 presents a schematic view of the CMS detector.

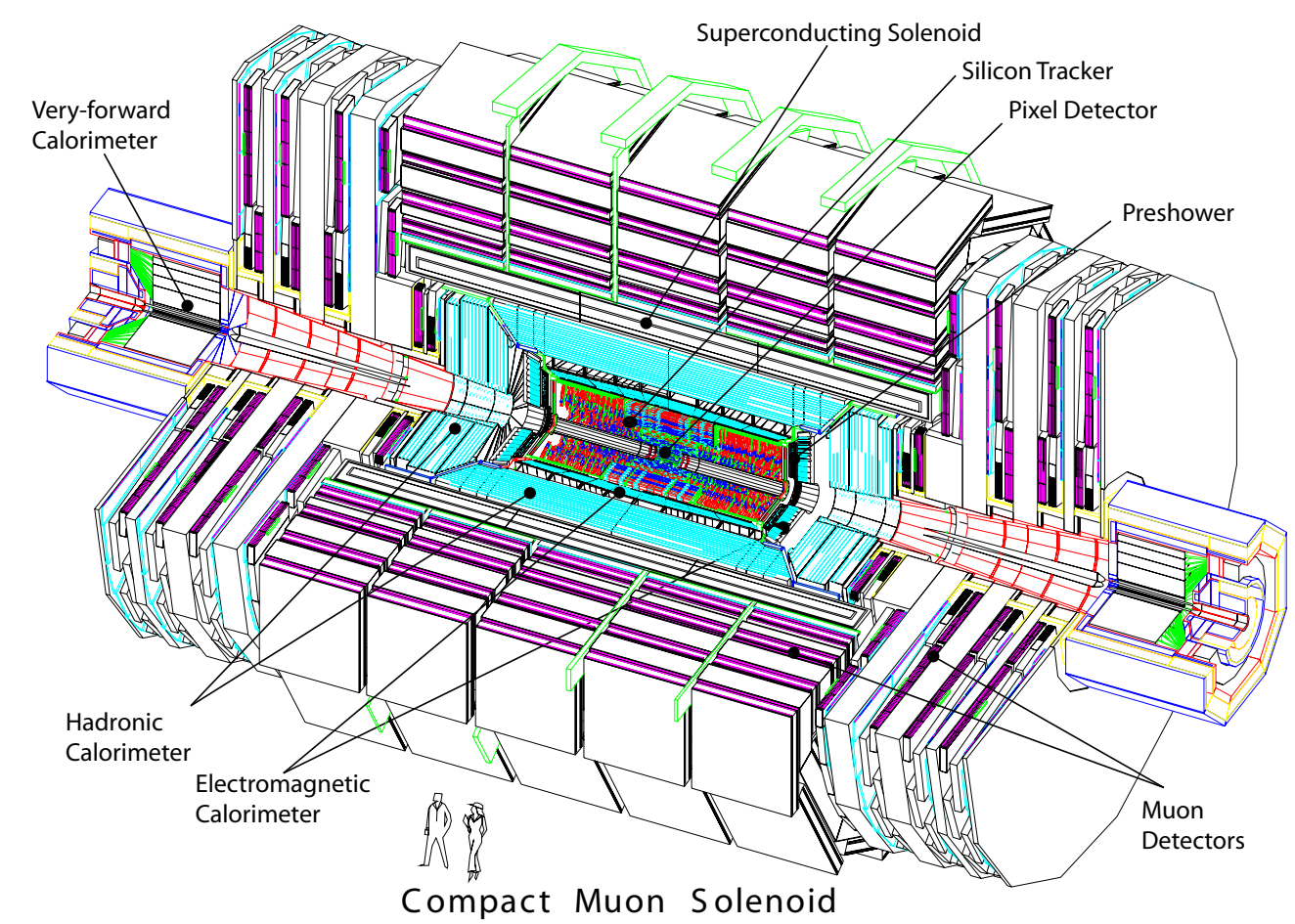

Figure 2.2: Schematic view of the CMS detector. 


\subsubsection{Coordinate Conventions}

The CMS coordinate system is oriented such that the $x$-axis points south to the center of the LHC ring, the $y$-axis points vertically upward and the $z$-axis is in the direction of the beam to the west. The azimuthal angle $\phi$ is measured from the $x$-axis in the $x y$ plane and the radial coordinate in this plane is denoted by $r$. The polar angle $\theta$ is defined in the $r z$ plane and the pseudorapidity is $\eta=-\ln \tan (\theta / 2)$. The momentum component transverse to the beam direction, denoted by $p_{T}$, is computed from the $x$ - and $y$-components, while the transverse energy is defined as $E_{T}=E \sin \theta$.

\subsubsection{Solenoid}

A superconducting solenoid magnet with a maximum magnetic field of $3.8 \mathrm{~T}$ provides the large bending power for high-energy charged particles to precisely measure their momentum in the tracking detectors. The magnetic coil is $13 \mathrm{~m}$ long, has an inner diameter of $6 \mathrm{~m}$ and accommodates the tracking and part of the calorimeter detectors. With these dimensions the CMS solenoid is the largest superconducting magnet ever built and has the capacity to store an energy of 2.6 GJ at full current. The magnetic flux is returned through a $10000 \mathrm{t}$ iron yoke in which the muon detector chambers are integrated. The CMS solenoid was fully tested and commissioned at the experimental surface hall during autumn 2006.

\subsubsection{Tracking Detectors}

CMS features an all silicon tracker with a total active area of $200 \mathrm{~m}^{2}$. The tracking detector is divided into a pixel detector close to the interaction region and a strip detector covering radii between $0.2 \mathrm{~m}$ and $1.2 \mathrm{~m}$. At LHC design luminosity more than 1000 particles are expected to traverse the tracking volume in each bunch crossing. This leads to a hit rate density of $1 \mathrm{MHz} / \mathrm{mm}^{2}$ at a radius of $4 \mathrm{~cm}$ which imposes severe challenges to the design of the tracking detectors. With a pixel size of $100 \times 150 \mu \mathrm{m}^{2}$ an occupancy of less than $10^{-4}$ can be maintained in the pixel detector. As the particle flux decreases with the distance from the interaction point, sensors with a length of $10 \mathrm{~cm}$ and a pitch of $80 \mu \mathrm{m}$ can be used at intermediate radii $(20-55 \mathrm{~cm})$ and sensors with a length of $25 \mathrm{~cm}$ and a pitch of $180 \mu \mathrm{m}$ at the outermost radii $(55-110 \mathrm{~cm})$ with an occupancy of less than $3 \%$. The sensor thickness is $285 \mu \mathrm{m}$ for the pixel and $320 \mu \mathrm{m}$ or $500 \mu \mathrm{m}$ for the strip modules at the intermediate and outer radii, respectively. The thicker sensors for the outer tracking region permit to preserve a signal to noise ratio well above 10 in spite of the higher noise due to the larger capacity of the longer strips. To mitigate the radiation damage effects and prolong the lifetime of the detector modules, the tracking detectors are designed to run at subzero temperatures. The cooling is established using a mono-phase liquid cooling system whit $\mathrm{C}_{6} \mathrm{~F}_{14}$ as cooling fluid.

The pixel detector is built from 3 barrel layers at radii of $4.4,7.3$ and $10.2 \mathrm{~cm}$ and two end disks on each side at a distance of $z= \pm 34.5, \pm 46.5 \mathrm{~cm}$ from the interaction point. It consists of 1440 segmented silicon sensor modules with a total of 66 million readout channels. For each pixel the analog pulse height information is detected and read out.

The sensor surface of the barrel modules is parallel to the magnetic field, while the modules in the forward detector are tilted by $20^{\circ}$. The charge carriers produced by a particle traversing the sensor thus experience the Lorentz force and do not drift along the electric field line anymore. Hence, the charge carriers are distributed over several pixels. The analog pulse height information can be used to calculate a center of gravity of the charge distribution 
which improves the hit information. In this way a position resolution of about $15 \mu \mathrm{m}$ in both the $r \phi$ and $z$ directions is obtained (compare to figure 2.6). A detailed description of the design and the functioning of the CMS pixel barrel detector is given in chapter 7 .

The silicon strip tracker has a length of $5.8 \mathrm{~m}$ and a diameter of $2.4 \mathrm{~m}$ and is composed of four subsystems: the four-layer Tracker Inner Barrel (TIB), the six-layer tracker outer barrel (TOB) and on each side three-disk Tracker Inner Disks (TID) and nine-disk Tracker Endcaps (TEC). An $r z$-view of the tracker geometry is shown in figure 2.3.

The silicon strip tracker is built from 15148 single-sided modules that provide 9.3 million readout channels. Modules for the TIB, the TID and the first four rings of the TEC are single-sided while the TOB and the outer three rings of the TEC are equipped with doublesided modules. A double-sided module is constructed from two single-sided modules glued back-to-back at a stereo angle of $100 \mathrm{mrad}$.

\subsubsection{Track Reconstruction}

Due to the magnetic field charged particles travel through the tracking detectors on a helix trajectory which is described by 5 parameters: the curvature $\kappa$, the track azimuthal angle $\phi$ and polar angle $\eta$, the signed transverse impact parameter $d_{0}$ and the longitudinal impact parameter $z_{0}$. The transverse (longitudinal) impact parameter of a track is defined as the transverse (longitudinal) distance of closest approach of the track to the primary vertex.

The main standard algorithm used in CMS for track reconstruction is the Combinatorial Track Finder (CFT) algorithm [17] which uses the reconstructed positions of the passage of charged particles in the silicon detectors to determine the track parameters. The CFT algorithm proceeds in three stages: track seeding, track finding and track fitting. Track candidates are best seeded from hits in the pixel detector because of the low occupancy, the high efficiency and the unambiguous two-dimensional position information.

The track finding stage is based on a standard Kalman filter pattern recognition approach [18] which starts with the seed parameters. The trajectory is extrapolated to the next

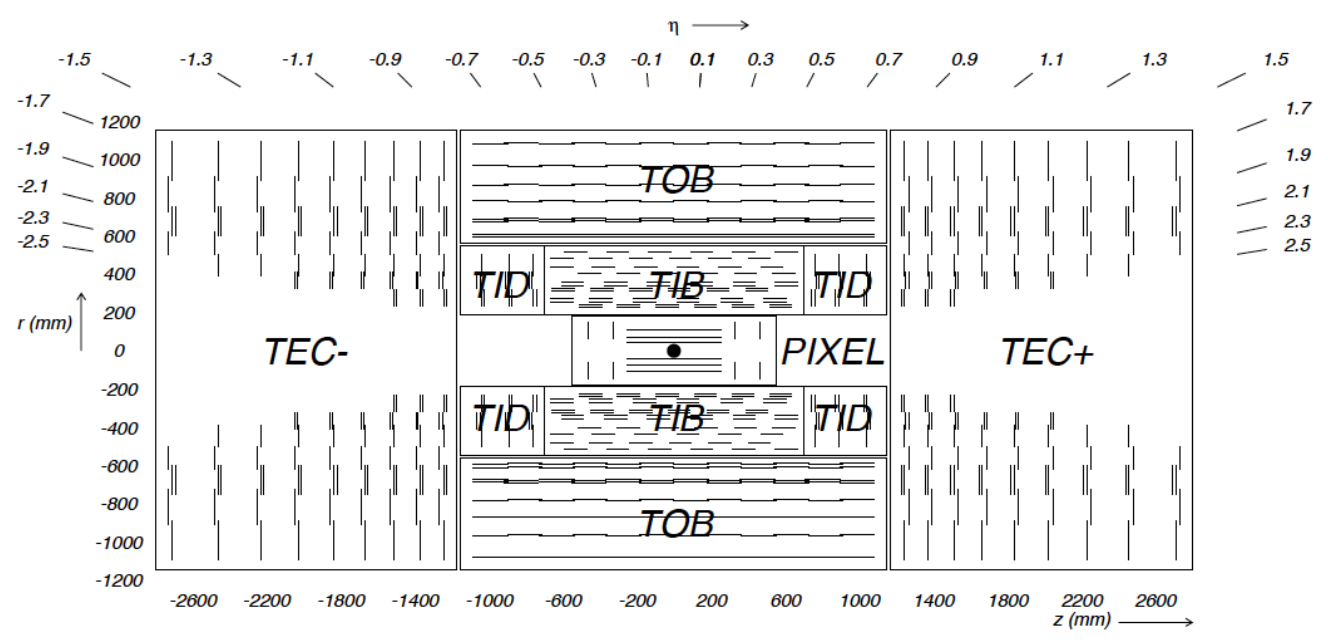

Figure 2.3: $r z$-view of the CMS tracking detectors [14]. Single lines represent layers of modules equipped with one sensor, double lines indicate layers with back-to-back modules. 
tracker layer and compatible hits are assigned to the track on the basis of the $\chi^{2}$ between the predicted and measured positions. At each stage the Kalman filter updates the track parameters with the new hits. In order to take into account possible inefficiencies one further candidate is created without including any hit information. The tracks are assigned a quality based on the $\chi^{2}$ and the number of missing hits and only the best quality tracks are kept for further propagation. Ambiguities between tracks are resolved during and after track finding. In case two tracks share more than $50 \%$ of their hits, the lower quality track is discarded.

For each trajectory the finding stage results in an estimate of the track parameters. However, since the full information is only available at the last hit and constraints applied during trajectory building can bias the estimate of the track parameters, all valid tracks are refitted with a standard Kalman filter and a second filter (smoother) running from the exterior towards the beam line.

The expected performance of the track reconstruction is shown in figure 2.4 for muons, pions and hadrons. The track reconstruction efficiency for high energy muons is about $99 \%$ and drops at $|\eta|>2.1$ due to the reduced coverage of the forward pixel detector. For pions and hadrons the efficiency is in general lower because of interactions with the material in the tracker. The material budget of the CMS tracker in units of radiation length ${ }^{1}$ is presented in figure 2.5.
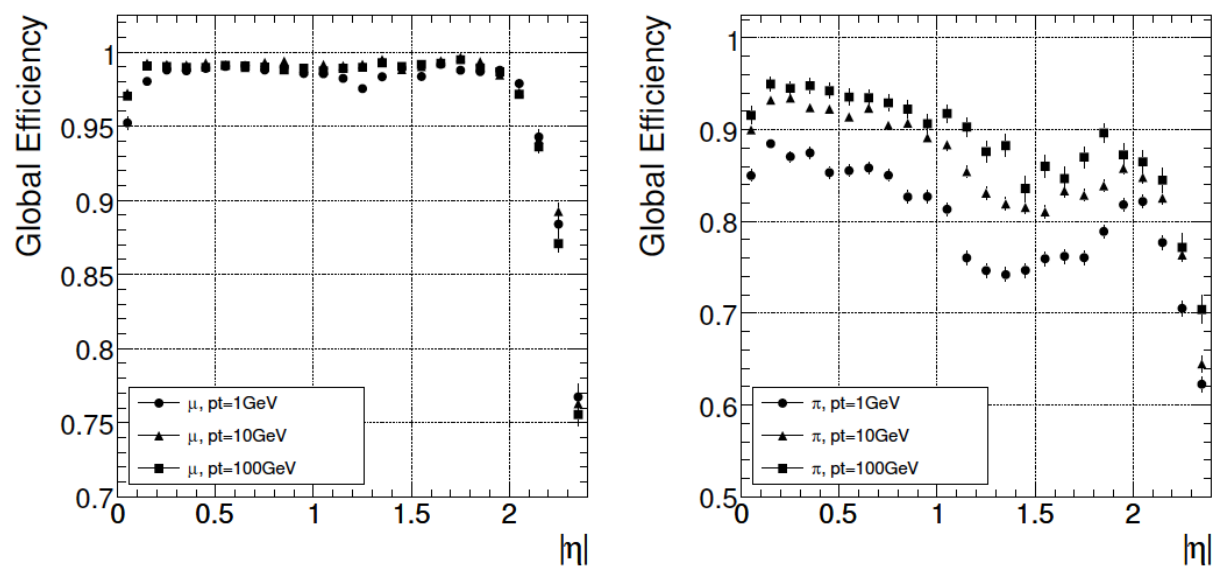

Figure 2.4: Track reconstruction efficiency for muons (left) and pions (right) with transverse momenta of 1,10 and $100 \mathrm{GeV}$ [14].

In figure 2.6 the transverse momentum resolution for muon tracks with $p_{T}=1,10$ and $100 \mathrm{GeV}$ is shown. At high momenta the resolution is around $1-2 \%$ for $|\eta|<1.6$. The material of the tracker accounts for $20-30 \%$ of the transverse momentum resolution. At lower momenta, the resolution is dominated by multiple scattering and its distribution reflects the amount of material traversed by the track. The resolution of the track impact parameters in the longitudinal and the transverse plane are also shown in figure 2.6. At high momentum the transverse impact parameter resolution is fairly constant and is dominated by the hit resolution in the first pixel layer. It is progressively degraded by multiple scattering at lower momenta. The same applies to the longitudinal impact parameter resolution. The

\footnotetext{
${ }^{1}$ The radiation length $X_{0}$ is a characteristic of a material, related to the energy loss of high energy electrons in the material.
} 


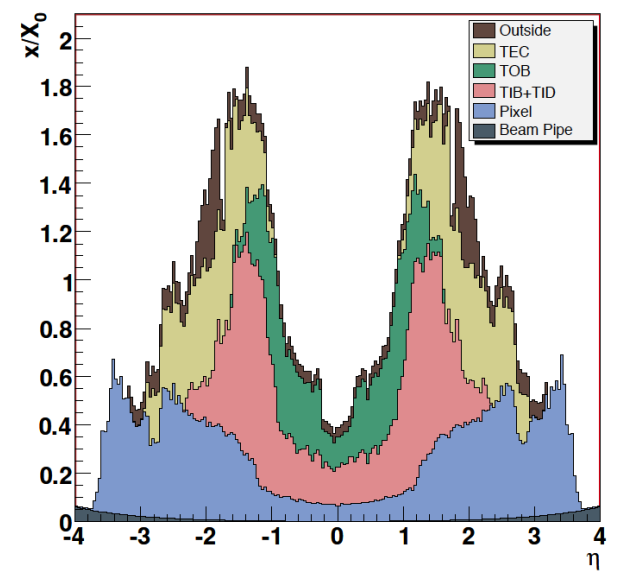

Figure 2.5: Material budget of the CMS tracker in units of radiation length $X_{0}$ as a function of pseudorapidity divided into the contributions of the different subdetectors [14].

improvement of the $z_{0}$ resolution up to $|\eta|=0.5$ is due to the charge sharing effects among neighboring pixels.
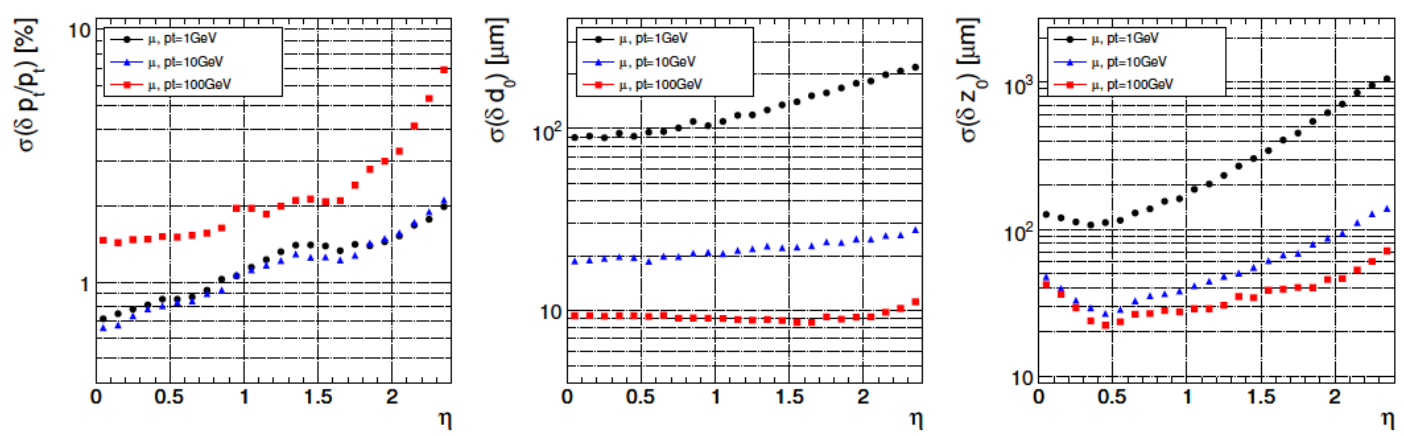

Figure 2.6: Resolution of track transverse momentum (left), transverse impact parameter (middle) and longitudinal impact parameter (right). The resolution is shown for muons with transverse momenta of 1,10 and $100 \mathrm{GeV}$ [14].

\subsubsection{Electromagnetic Calorimeter}

The CMS electromagnetic calorimeter (ECAL) is a hermetic and homogeneous detector with a large pseudorapidity coverage up to $|\eta|<3$. The ECAL is divided into barrel and endcap detectors as illustrated in figure 2.7. Scintillation crystals made from lead tungstate $\left(\mathrm{PbWO}_{4}\right)$ are used to measure the energy of electromagnetically interacting particles, mainly electrons and photons. The choice of $\mathrm{PbWO}_{4}$ as the material for the scintillation crystal is motivated by its fast response time and high radiation resistance. The ECAL consists of 61200 crystals 
in the barrel and 7324 crystals in the endcaps. The crystals have a tapered shape and are mounted in a quasi-projective geometry. With a crystal front face of $22 \times 22 \mathrm{~mm}^{2}$ and $28.6 \times 28.6 \mathrm{~mm}^{2}$ in the barrel and the endcaps, respectively, a fine granularity is ensured. The length of a barrel crystal is $23 \mathrm{~cm}$ which corresponds to $25.8 \mathrm{X}_{0}$, while the endcap crystals are $22 \mathrm{~cm}$ long corresponding to $24.7 \mathrm{X}_{0}$. The scintillation light produced in the crystals is read out by avalanche photo diodes (APD) with an active area of $5 \times 5 \mathrm{~mm}^{2}$ in the barrel and by vacuum phototriodes (VPT) with an active area of $280 \mathrm{~mm}^{2}$ in the endcaps. The light output and the amplification have a strong temperature dependence. The response to an incident electron changes by $(3.8 \pm 0.4) \% /{ }^{\circ} \mathrm{C}$ which in turn means that the temperature has to be closely monitored and kept stable to a precision of $\pm 0.05{ }^{\circ} \mathrm{C}$. The nominal operating temperature of the ECAL is $18^{\circ} \mathrm{C}$ and is provided by a water cooling system.

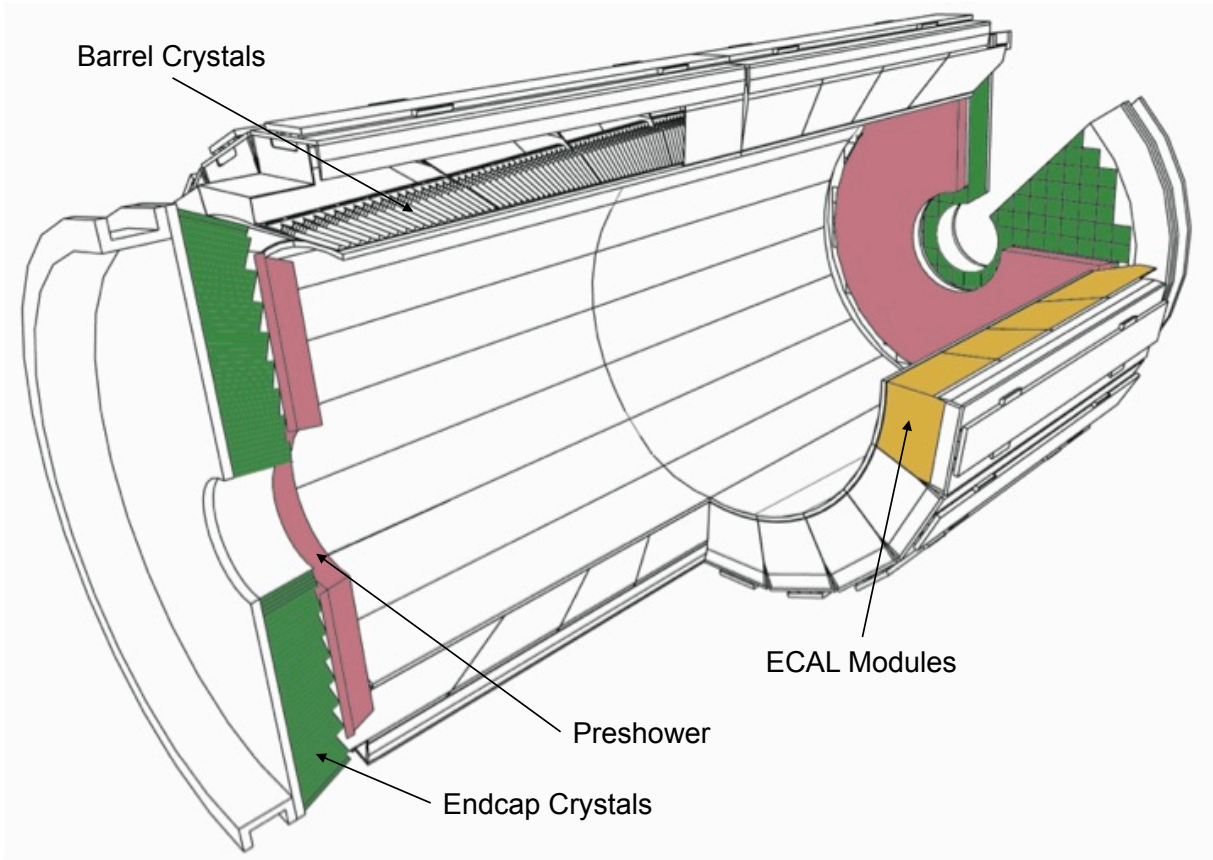

Figure 2.7: Schematic view of the CMS Electromagnetic Calorimeter [14].

The ECAL barrel has a volume of $8.14 \mathrm{~m}^{3}$ and its front face is at a radial distance of $1.29 \mathrm{~m}$ from the interaction point. It has a 360 -fold azimuthal segmentation and two times 85 -fold segmentation in pseudorapidity. The endcap has a coverage of $1.479<|\eta|<3$ and is situated at a longitudinal distance of $3.15 \mathrm{~m}$ from the interaction point. A preshower detector with a thickness of $3 X_{0}$ is placed in front of the endcaps $(1.653<|\eta|<2.6)$ to guarantee a reliable discrimination of single photons and photons produced in pairs in neutral pion decays.

The energy resolution of the electromagnetic calorimeter can be parametrized by the following expression:

$$
\left(\frac{\sigma}{E}\right)^{2}=\left(\frac{S}{\sqrt{E}}\right)^{2}+\left(\frac{N}{E}\right)^{2}+C^{2}
$$

where $S$ is the stochastic term, $N$ the noise term and $C$ the constant term. The value 
of the three parameters were determined by a electron test beam measurement to be $S=$ $0.028 \mathrm{GeV}^{\frac{1}{2}}, N=0.12 \mathrm{GeV}$ and $C=0.003$. The ECAL energy resolution as a function of the energy of electrons is shown in figure 2.8 .

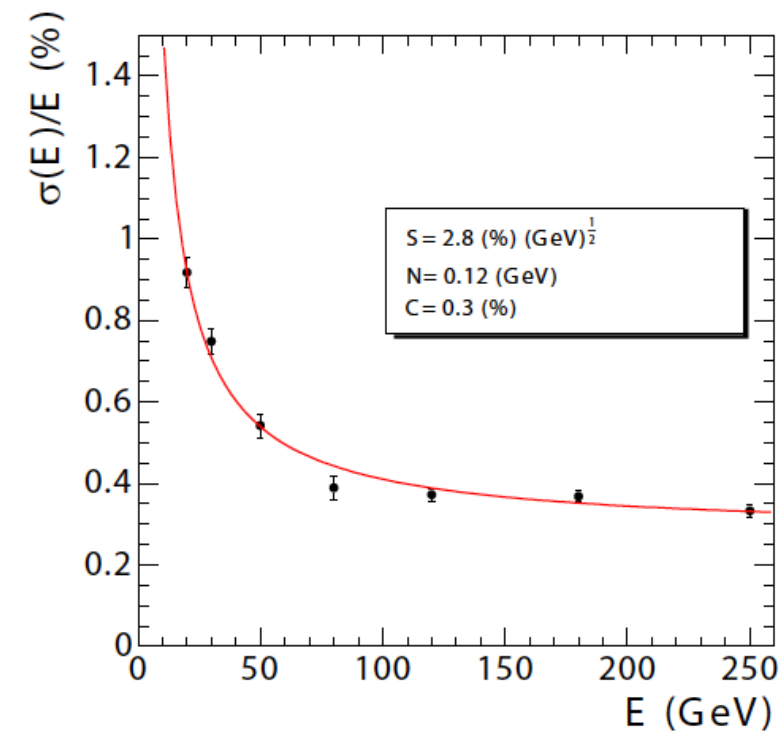

Figure 2.8: ECAL energy resolution as a function of the energy measured in an electron test beam [14]. The measured values of the stochastic (S), noise (N) and constant (C) term are displayed in the legend.

\subsubsection{Hadronic Calorimeter}

The energy measurement of the ECAL is complemented by the measurement of the hadronic calorimeter (HCAL). The HCAL is a sampling calorimeter built from alternating layers of massive absorbing brass plates and plastic scintillator tiles arranged in trays. The quality of the energy measurements depends on the fraction of the hadronic shower detected in the calorimeter. Consequently, the thickness of the material in the tray has to be large enough to absorb the major part of the shower. The dimensions of the barrel part of the HCAL however are restricted to the limited volume between the outer extent of the ECAL $(r=1.77 \mathrm{~m})$ and the inner extent of the magnetic coil $(r=2.95 \mathrm{~m})$. Thus, the HB is supplemented by an outer hadronic calorimeter (HO) located between the solenoid and the muon detectors. The $\mathrm{HO}$ uses the solenoid as additional absorbing material and provides sufficient containment for hadronic showers with a thickness of 11.8 interaction lengths $\left(\lambda_{l}\right)$. The hadronic calorimeter is divided into a barrel part (HB and $\mathrm{HO}$ ) at $|\eta|<1.3$, an endcap (HE) on each side at $1.3<|\eta|<3$ and a forward calorimeter (HF) extending up to $|\eta|<5.2$ to achieve a most hermetic detector coverage. The HCAL tower segmentation in the $r z$ plane for one quarter of the HB, HO and HE detectors is shown in figure 2.9 and summarized in table 2.1.

The plastic scintillator tiles are read out by wavelength shifting fibers that shift the blueviolet light emitted by the scintillator to green light which is then sent through transparent 


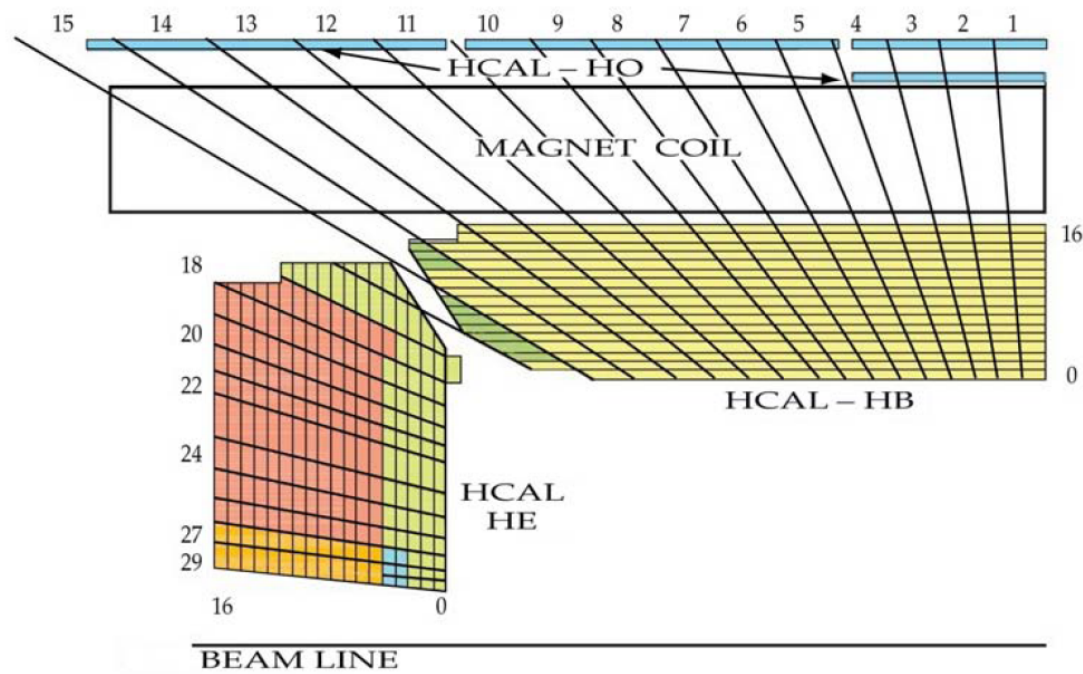

Figure 2.9: Tower segmentation for one quarter of the HCAL displayed in the $r z$ plane [14]. The colors represent the optical grouping of scintillator layers into different longitudinal readouts.

Table 2.1: Tower segmentation in azimuthal and polar angle for the hadronic barrel, endcap and forward calorimeter.

\begin{tabular}{cccccc}
\hline & $\mathrm{HB} / \mathrm{HO}$ & $\mathrm{HE}|\eta| \leqslant 2.5$ & $\mathrm{HE}|\eta|>2.5$ & $\mathrm{HF}|\eta| \leqslant 4.7$ & $\mathrm{HF}|\eta|>4.7$ \\
\hline$\Delta \phi \times \Delta \eta$ & $0.087 \times 0.087$ & $0.087 \times 0.087$ & $0.175 \times 0.175$ & $0.175 \times 0.175$ & $0.175 \times 0.35$ \\
\hline
\end{tabular}

fibers to hybrid photodetectors (HPDs) with 19 independent pixels. The first scintillators are placed in front of the first absorber plate in order to sample showers developing in the material between the ECAL and the HCAL, while the last scintillators are installed after the last absorber plate to correct for late developing showers leaking out. 70000 and 20916 scintillator tiles are installed in the HB and the HE, respectively.

The HF is positioned at a longitudinal distance of $11.2 \mathrm{~m}$ from the interaction point. It will experience unprecedented particle fluxes with an energy of $760 \mathrm{GeV}$ deposited on average in a proton-proton interaction at $\sqrt{s}=14 \mathrm{TeV}$. This energy has to be compared to the average of $100 \mathrm{GeV}$ deposited in the rest of the detector. The situation is even more severe as the energy is not spread equally among the HF, but has a pronounced peak at the highest rapidity. The $\mathrm{HF}$ is made from steel absorber plates composed of $5 \mathrm{~mm}$ thick grooved plates with quartz fibers inserted as active medium. It detects the Cherenkov light emitted by charged particles in the shower and is thus mainly sensitive to the electromagnetic component of the shower. A longitudinal segmentation in two parts allows to distinguish signals generated by electrons and photons from signals generated by hadrons.

\subsubsection{Muon System}

The muon system is the outermost part of the CMS detector. The magnet return yoke is equipped with gaseous detector chambers for muon identification and momentum measure- 
ment. In the barrel, the muon stations are arranged in five separate iron wheels and in the endcap, four muon stations are mounted onto the three independent iron disks in the positive and the negative endcaps. Each barrel wheel is segmented into 12 sectors in azimuthal angle.

Three different types of gaseous detectors are integrated into the CMS muon system depending on the requirements. In the barrel part where both the muon rate and the neutron induced background are small and the magnetic field is very low, drift tube (DT) chambers are used. In the endcaps however the muon and the background flux is much higher. The muon detector endcaps are thus built from cathode strip chambers (CSCs) which provide a faster response, a higher granularity and a better resistance against radiation. In addition, resistive plate chambers (RPCs) form a redundant trigger system. In total, the CMS muon system consists of 250 DT chambers, 540 CSCs and 610 RPCs. The arrangement of the detector chambers is shown in figure 2.10 .

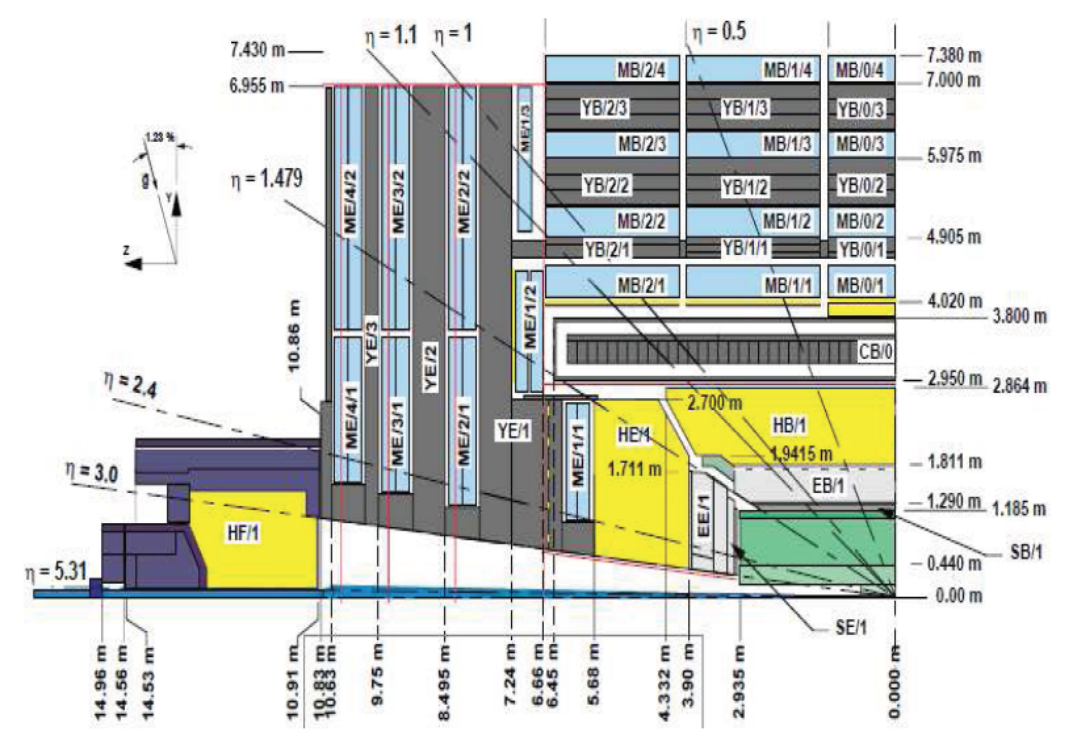

Figure 2.10: Longitudinal view of one quarter of the CMS detector illustrating the layout of the muon system in the barrel and the endcap region [14].

A DT cell is a $4 \mathrm{~cm}$ wide gas tube with a positively charged stretched wire inside. Each DT chamber, on average $2 \mathrm{~m} \times 2.5 \mathrm{~m}$ in size, consists of 12 layers of DT cells, arranged in three groups of four. The middle group measures the $z$ coordinate while the two outside groups measure the $r \phi$ coordinate. In the barrel, four DT chambers are interspersed with the layers of the flux return yoke in each $\phi$ sector. The outermost muon station is equipped with DT chambers that contain only 8 layers of DT cells and determine the muon position in the $r \phi$ plane. The barrel part of the muon system covers the region $|\eta|<1.2$.

The CSCs are trapezoidal shaped multiwire proportional chambers which consist of 6 anode wire planes crossed with 7 copper strips cathode panels in a gas volume. They provide a two-dimensional position measurement, where the $r$ and $\phi$ coordinates are determined by the copper strips and the anode wires, respectively. The muon detector endcaps consist of 4 CSC stations on each side and identify muons in the pseudorapidity range of $0.9<|\eta|<2.4$.

RPCs are made from two high resistive plastic plates with a voltage applied and separated 
by a gas volume. The signal generated by the muon when passing through the gas volume is detected by readout strips mounted on top of one of the plastic plates. The RPCs used in the muon trigger system are highly segmented and have a fast response with a time resolution of $1 \mathrm{~ns}$. Six layers of RPCs are installed in the barrel muon system, two layers in each of the first two stations and one layer in each of the last two stations. One layer of RPCs is built into each of the first three stations of the endcap.

\subsubsection{Muon Reconstruction}

Muon reconstruction, after local-pattern recognition is performed in two stages: stand-alone reconstruction based on information from the muon system only and global reconstruction including the hit information of the silicon tracker. Stand-alone reconstruction starts from track segments in the muon chambers and muon trajectories are built from the inside to the outside using the Kalman filter technique. After the trajectory is built, a second Kalman filter, working from outside in, is applied to determine the track parameters. In the end, the track is extrapolated to the nominal interaction point and a vertex-constrained fit of the track parameters is performed.

In the global muon reconstruction the muon trajectories are extended to add hits measured by the tracker. The track parameters of a stand-alone reconstructed muon are compared to the track parameters of the tracker tracks by extrapolating the trajectories to a common plane on the inner surface of the muon detector. If a tracker track is found that is compatible in momentum, position and direction, the hit information of the tracker and the muon system is combined and refitted to form a global muon track. The resulting global tracks are then checked for ambiguity and quality to choose at most one global track per stand-alone muon.

The precision of the momentum measurement in the muon system is essentially determined by the measurement of the bending angle in the transverse plane at the exit of the magnetic coil. This measurement is dominated by multiple scattering in the material before the first muon station up to transverse momentum values of $200 \mathrm{GeV}$. For low-momentum muons the momentum resolution is improved substantially by including the measurement of the silicon tracker. The analysis presented here investigates muons with a transverse momentum between 6 and $30 \mathrm{GeV}$. The inclusion of the tracker information by using global muons is thus most valuable. In figure 2.11 a comparison of the momentum resolution of the muon system, the tracker system and a combined measurement is given for the barrel and the forward region.

\subsubsection{Trigger System}

The CMS trigger system is designed to cope with the unprecedented high luminosity and interaction rates. It must ensure high data recording efficiency for a wide variety of physics objects and event topologies, while applying online selective requirements to reduce the $40 \mathrm{MHz}$ event rate to an output rate of about $100 \mathrm{~Hz}$ allowing for permanent storage of an event.

The CMS trigger system reduces the event rate in two steps called Level 1 (L1) and High Level Trigger (HLT). The L1 trigger is designed to achieve a maximum output rate of $100 \mathrm{kHz}$ and consists of custom-designed, programmable electronics while the HLT is based on software algorithms running on a large cluster of commercial processors, the event filter farm.

The L1 trigger system uses only coarsely segmented data from the muon system and the calorimeters while the full granularity data are stored in the detector front-end electronics 

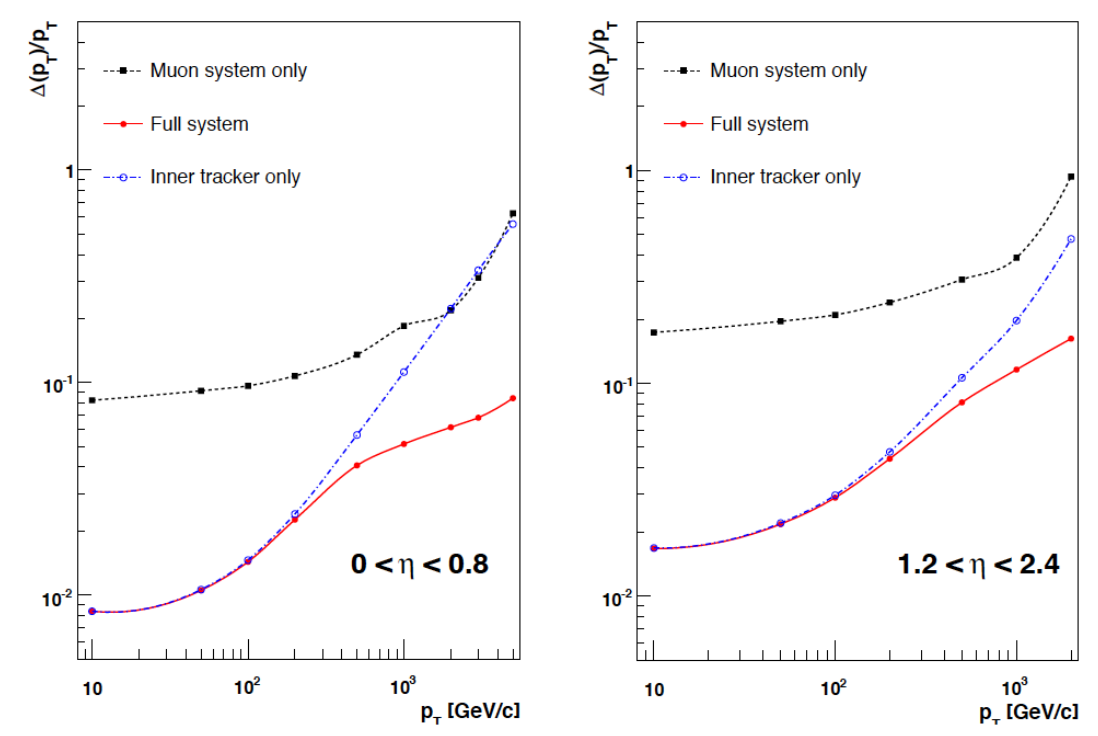

Figure 2.11: Muon transverse momentum resolution as a function of the transverse momentum for muons detected in the barrel (left) and the endcap (right) regions [14]. The resolution is given for the measurement using the muon system or the tracking system only and for a combined method.

waiting for the L1 decision. The L1 decision has to be taken within a latency time of $3.2 \mu \mathrm{s}$ and is based on the decision of local, regional and global trigger components. It also depends on the readiness of the other subdetectors and the data acquisition system (DAQ) which is supervised by the Trigger Control System. The trigger architecture is displayed in figure 2.12.

The ECAL and the HCAL cells form trigger towers with an $(\eta, \phi)$ coverage of $0.087 \times 0.087$ in $|\eta|<1.74$ and larger size in the forward region. Trigger primitives are generated by calculating the transverse energy of a trigger tower and assigning it to the correct bunch crossing. A regional calorimeter trigger then determines regional electron, photon and jet candidates and information relevant for muon and tau identification. The global calorimeter trigger provides information about the jets, the total transverse energy and the missing energy in the event and identifies the highest-ranking trigger candidates.

In the muon system all three types of detectors take part in the trigger decision. The DT chambers provide track segments in the $\phi$ projection and hit pattern in $\eta$, while the CSC determine three-dimensional track segments. The track finders in the DT chambers and the CSCs calculate the transverse momentum of a track segment and its location and quality. The RPCs deliver an independent measurement derived from regional hit patterns. The global muon trigger receives up to four candidates from each subsystem (DT, barrel RPC, CSC and endcap RPC) together with the isolation information from the global calorimeter trigger. The aim is to improve the efficiency and to reduce the rate by making use of the complementarity and the redundancy of the subsystems. In the end, the global muon trigger selects a maximum of four muon trigger candidates and determines their momentum, charge, position and quality.

The trigger objects extracted by the global calorimeter trigger and the global muon trigger 


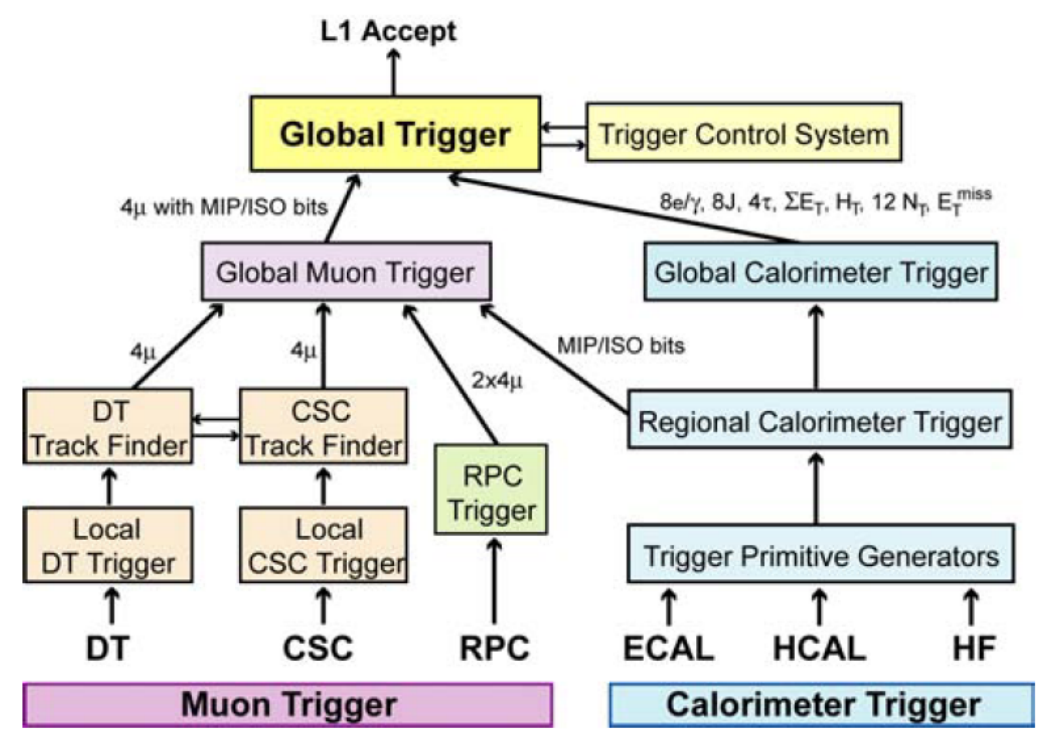

Figure 2.12: L1 trigger architecture [14].

are sent to the global trigger where the decision to accept or reject an event is taken and distributed to the subdetectors. The decision is based on the results of algorithms which for example apply momentum thresholds to single objects or require object multiplicities to exceed predefined values. Up to 128 algorithms can be executed in parallel.

If an event is accepted by the L1 trigger, the full detector information $(\sim 1 \mathrm{MB})$ is read out by the DAQ system at a rate of up to $100 \mathrm{kHz}$, passed to the event filter farm and used as input for the HLT. The HLT algorithms are implemented in the same software as used for offline reconstruction and analysis and consist of subsequent reconstruction and selection steps. The framework uses a modular architecture and the modules to be executed are defined at runtime by means of configuration files. The HLT menu is composed of a set of trigger paths, each path addressing a specific physics object selection. The execution of a path is interrupted if the processed event does not fulfill the conditions imposed by a given filter module. The trigger menu for the first CMS data taking can be found in [19]. 


\section{Part I}

\section{Study of the Inclusive $b$ Quark Production at CMS}





\section{Chapter 3}

\section{Heavy Flavor Physics}

The study of heavy quark production is an important research area at the LHC. Heavy quarks will be produced with a large cross section at a yet unreached center-of-mass energy, enabling precision measurements to improve our understanding of heavy flavor physics. In the context of this work the term heavy quark stands for charm and beauty quarks since the mass of the up, down and strange quark are significantly lower. The heavier top quark has a very short lifetime and does therefore not form bound states of heavy hadrons.

Heavy quark production is interesting on its own as it presents a key process for the study of the theory of strong interactions, Quantum Chromodynamics (QCD). Furthermore, a well-established theory of heavy quark production is needed for many searches at the LHC.

In this chapter the theoretical concepts relevant to describe the physics of heavy quarks at the LHC are introduced. The main ideas of Quantum Chromodynamics are reviewed, before their application to high-energy hadron-hadron collisions is discussed. This includes the factorization ansatz, the evolution of the parton distribution functions, the partonic processes important for beauty quark production and the phenomenological treatment of heavy quark fragmentation. A further section is dedicated to the description of the decay of $b$-hadrons via the weak interaction. The Monte Carlo event generators which are used in this analysis to generate full hadronic events within the QCD framework are presented in the last section.

\subsection{Quantum Chromodynamics}

Quantum Chromodynamics $[20,21,22,23]$ is the field theory describing the strong interaction between color charged partons. Color charge comes in three versions (red, green and blue) which form a fundamental representation of the $S U(3)$ symmetry group and is carried by massive spin- $\frac{1}{2}$ quarks and massless spin- 1 gluons. Analogous to the photons in Quantum Electrodynamics (QED) [24, 25], the gluons are the gauge bosons in QCD and mediate the strong interaction. Since the gluons themselves carry color charge, they can directly interact with other gluons. This possibility is not available in QED, as photons do not have an electric charge. The existence of direct coupling in QCD has important implications on the scale dependence of the strong coupling. A comprehensive overview of QCD applied to hadronic collisions is given in $[26,27]$.

In QCD, as in any renormalizable quantum field theory, ultraviolet divergences appearing in the calculation can be removed by introducing a scale dependent coupling $\alpha_{s}\left(Q^{2}\right)$ and a new scale, the renormalization scale $\mu_{R}$ [28]. The dependence of $\alpha_{s}$ on the energy-scale $Q^{2}$ is 


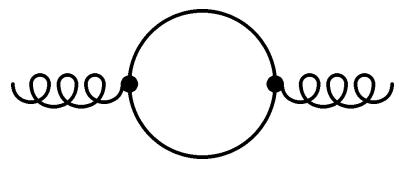

(a)

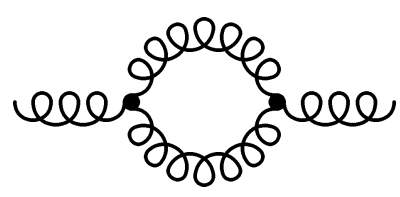

(b)

Figure 3.1: Graphs which contribute to the $\beta$ function in the one loop approximation: (a) fermion loop, (b) gluon loop.

known as running of the coupling. The requirement that every physical observable has to be independent of the arbitrary choice of $\mu_{R}$ leads to the renormalization group equation

$$
Q^{2} \frac{\partial \alpha_{s}\left(Q^{2}\right)}{\partial Q^{2}}=\beta\left(\alpha_{s}\right)
$$

While the running of the coupling is determined by the $\beta$ function in the above equation, the absolute value of $\alpha_{s}$ has to be determined experimentally. In perturbative QCD (pQCD), the $\beta$ function can be written as a perturbation series with $\alpha_{s}$ as expansion parameter. The coefficients of the expansion series are extracted from higher order diagrams, such as the ones shown in figure 3.1. Due to the diagrams representing the color self-coupling of the gluons the leading coefficients in QED and QCD have opposite signs. This sign is the origin of the most fundamental difference between QCD and QED. The leading order approximation gives the solution

$$
\alpha_{s}\left(Q^{2}\right)=\frac{1}{b \ln \left(Q^{2} / \Lambda_{Q C D}^{2}\right)}, b=\frac{33-2 n_{f}}{12 \pi},
$$

where $n_{f}$ is the number of quark flavors with mass below $Q^{2}$, and $\Lambda_{Q C D}$ represents the scale at which the perturbative approach is not valid anymore since the coupling becomes too large. Equation (3.2) indicates that in QCD - unlike in QED - the coupling $\alpha_{s}$ decreases as the energy scale $Q^{2}$ increases. This phenomenon is known as asymptotic freedom and justifies the perturbative approach at energy scales $Q^{2}>\Lambda_{Q C D}$. Experimentally the value of $\Lambda_{Q C D}$ has been found to be of the order of $200 \mathrm{MeV}$. A summary of measurements of $\alpha_{s}$ as a function of the respective energy scale $Q$ is presented in figure 3.2. At the scale set by the mass of the $Z$ boson the average value of the strong coupling constant is $\alpha_{s}\left(M_{Z}\right)=0.1184 \pm 0.0007$ [29].

At energy scales below $\Lambda_{Q C D}$ the strong coupling rises to infinity, which becomes manifest in the confinement of quarks and gluons inside color-singlet hadrons. Quarks and gluons are not seen in experiments, instead they are transformed into observable hadrons in a process called fragmentation. In this regime a perturbative approach is not useful anymore.

Nonetheless perturbative calculations prove successful in the prediction of hadronic cross sections at sufficiently large energy scales. The reason is that the hadronization occurs at a significantly later time scale $\left(t \sim \frac{1}{\Lambda_{Q C D}}\right)$ than the production process $\left(t \sim \frac{1}{Q}\right)$ and therefore cannot influence the probability of the process to happen.

\subsection{Hadronic Collisions}

Due to the asymptotic freedom in QCD, the interaction between quarks and gluons becomes arbitrarily weak at short distances. Consequently hadrons behave as collections of free partons 


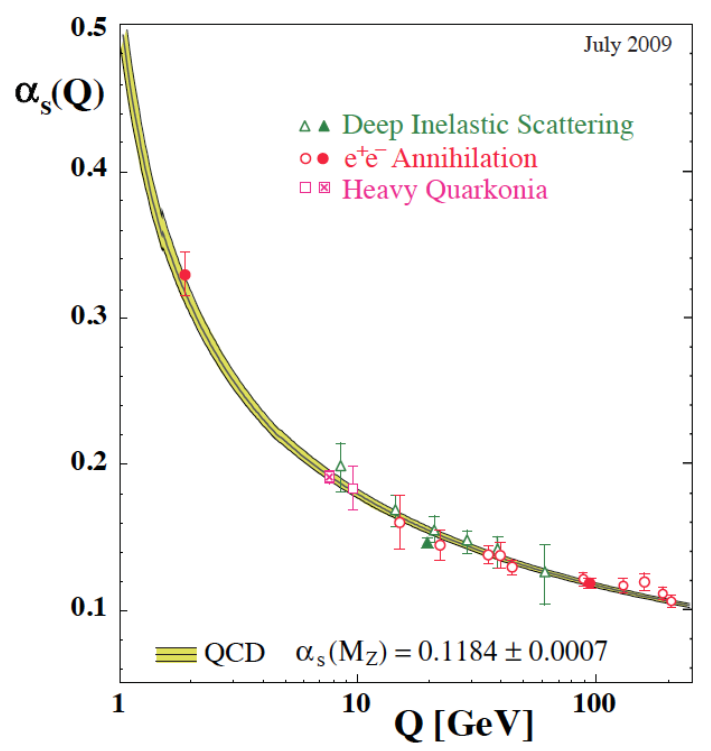

Figure 3.2: Summary of measurements of $\alpha_{s}$ as a function of the respective energy scale [29].

at large transferred momenta and their interaction can therefore be described using a parton model.

\subsubsection{Event Kinematics}

A generic scattering process of two hadrons $\left(h_{1}, h_{2}\right)$ with four-momenta $P_{1}$ and $P_{2}$, respectively, is illustrated in figure 3.3. The scattering process is caused by the interaction of two partons of the initial hadrons with four-momentum $p_{1}=x_{1} P_{1}$ and $p_{2}=x_{2} P_{2}$. Since the center-of-mass of the partonic interaction is normally boosted with respect to the laboratory frame, it is useful to classify the final state according to variables that are invariant under longitudinal boosts. The squared center-of-mass energy of the hadronic system is

$$
s=\left(P_{1}+P_{2}\right)^{2} .
$$

In the massless limit, the virtuality of the process is defined as

$$
Q^{2}=\hat{s}=x_{1} x_{2} s .
$$

The momentum imbalance of the partons participating in the hard interaction is reflected in the rapidity distribution of the outgoing particles. The transverse momentum of the outgoing partons in the center-of-mass frame of the colliding partons is denoted by $\hat{p}_{T}$ and is of particular interest for the Monte Carlo event generators (see section 3.6).

\subsubsection{Factorization}

Soft processes resulting in the production of low momentum hadrons will be the most common events in proton-proton collision at the LHC. Although these processes are QCD related, they cannot be calculated by pQCD. Perturbative approaches only lead to reliable results if a hard scale is present in the interaction. In the case of heavy flavor physics, the hard scale is 


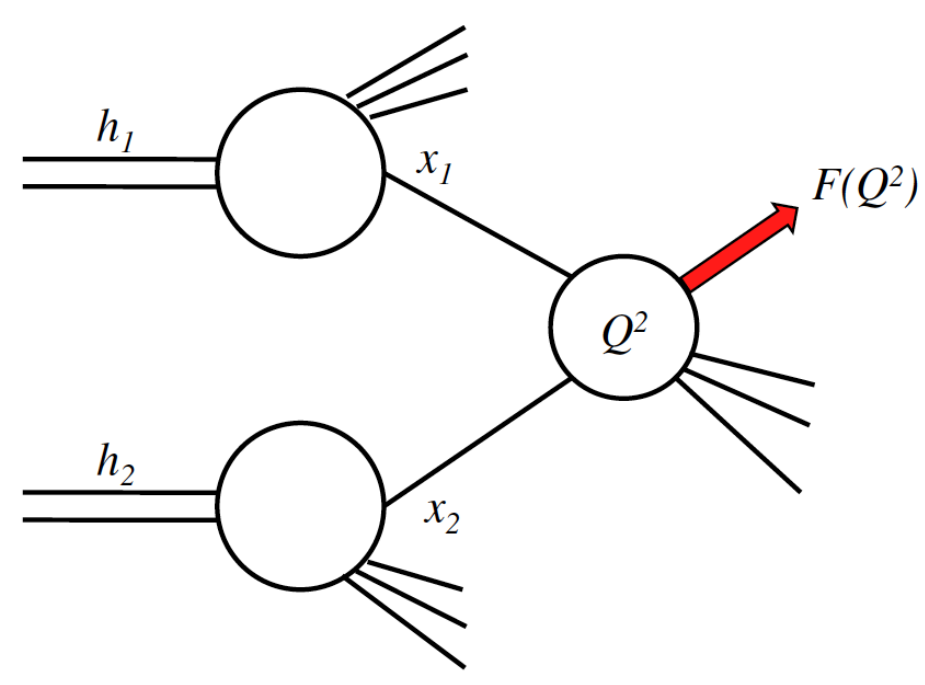

Figure 3.3: Scattering process of two hadrons $h_{1}$ and $h_{2}$ in the parton model. Two partons with momentum fractions $x_{1}$ and $x_{2}$ undergo a hard interaction at the scale $Q^{2}$.

provided by the mass of the heavy quark, its transverse momentum or the virtuality of the process.

Most of the processes calculated by pQCD feature infrared divergences emerging from real gluon emission. Singularities arise either if a gluon is emitted in the direction of the outgoing parton (collinear divergences) or if a low momentum gluon is emitted (soft divergence). Similar to the ultraviolet divergencies which are removed by introducing a renormalization scale, $\mu_{R}$, the infrared divergencies can be absorbed when imposing a factorization scale, $\mu_{F}$. The factorization scale can be thought of as the scale which separates the short- and the long-distance physics. The short-distance part covers the hard process calculable in pQCD, while the long-distance part includes the collinear and soft divergencies which are not accessible to perturbative calculations. The factorization ansatz is validated by the factorization theorem $[30,31,32]$.

According to the factorization theorem the cross section for a hard scattering originating from an interaction of two hadrons with four-momenta $P_{1}$ and $P_{2}$ can be written as

$\sigma\left(P_{1}, P_{2}\right)=\sum_{i, j} \int d x_{1} d x_{2} f_{i}^{h_{1}}\left(x_{1}, \mu_{F}^{2}\right) f_{j}^{h_{2}}\left(x_{2}, \mu_{F}^{2}\right) \hat{\sigma}_{i, j}\left(x_{1} P_{1}, x_{2} P_{2}, \alpha_{S}\left(\mu_{R}\right), Q^{2} ; \mu_{F}^{2}, \mu_{R}^{2}\right)$,

where

$f_{i}^{h}\left(x, \mu_{F}^{2}\right)$ is the parton distribution function (PDF) for the parton $\mathrm{i}$ in the hadron $\mathrm{h}$,

$x_{1} \quad$ is the momentum fraction of the hadron $h_{1}$ carried by the parton $\mathrm{i}$,

$x_{2} \quad$ is the momentum fraction of the hadron $h_{2}$ carried by the parton $\mathrm{j}$,

$\hat{\sigma}_{i, j} \quad$ is the short-distance scattering cross section of partons $\mathrm{i}$ and $\mathrm{j}$,

$\mu_{R} \quad$ is the renormalization scale, and

$\mu_{F} \quad$ is the factorization scale.

The parton distribution functions $f_{i}^{h}\left(x, \mu_{F}^{2}\right)$ describe the probability of extracting a parton 
$i$ from a hadron $h$ with momentum fraction $x$. Hence, the factorization theorem implies that the probability of extracting the parton can be treated independently from the parton undergoing an interaction. This assumption was successfully verified in deep inelastic leptonhadron scattering (DIS) which is characterized by a large virtuality $\left(Q^{2}>>\Lambda_{Q C D}\right)$. In the DIS regime, the factorization theorem is proven to be valid to all orders in perturbation theory [32]. Nonetheless it is not obvious that the factorization theorem can be adapted to hadron-hadron collisions since gluons from the hadron remnant might interact and spoil the factorization. Explicit calculations have shown that factorization breaking effects are present but are suppressed by powers of $\Lambda_{Q C D}$ in the high energy limit [33].

The partonic short-distance cross section $\hat{\sigma}_{i, j}$ can be computed in pQCD as

$$
\hat{\sigma}_{i, j}=\alpha_{s}^{k} \sum_{n}\left(\frac{\alpha_{s}}{\pi}\right)^{n} c^{(n)}
$$

Here the coefficients $c^{(n)}$ are functions of the kinematic variables and the factorization scale. Different hard processes will contribute with different leading powers $k$ to the partonic cross section.

\subsubsection{Evolution of Parton Distribution Functions}

As discussed in the previous section the long-distance, non-perturbative part of the cross section is absorbed in scale dependent PDFs which cannot be calculated by pQCD. Nevertheless, the dependence on the factorization scale $\mu_{F}$ is described by perturbative calculation. The pQCD parton evolution equations predict the evolution of the PDFs to any scale $Q^{2}>Q_{0}^{2}$ once $f_{i}^{h}\left(x, Q_{0}^{2}\right)$ is known at a starting scale $Q_{0}^{2}$. The scale dependence of the PDFs is a consequence of gluon radiation and gluon splitting effects, which are incorporated in the DGLAP evolution equations $[34,35,36,37]$ for the quark $\left(q_{i}\left(x, Q^{2}\right)\right)$ and gluon $\left(g\left(x, Q^{2}\right)\right)$ PDFs:

$$
\begin{gathered}
\frac{d q_{i}\left(x, Q^{2}\right)}{d \log Q^{2}}=\int_{x}^{1} \frac{d y}{y}\left(q_{i}\left(y, Q^{2}\right) P_{q q}\left(\alpha_{s}\left(Q^{2}\right), \frac{x}{y}\right)+g\left(y, Q^{2}\right) P_{q g}\left(\alpha_{s}\left(Q^{2}\right), \frac{x}{y}\right)\right) \\
\frac{d g\left(x, Q^{2}\right)}{d \log Q^{2}}=\int_{x}^{1} \frac{d y}{y}\left(\sum_{i} q_{i}\left(y, Q^{2}\right) P_{g q}\left(\alpha_{s}\left(Q^{2}\right), \frac{x}{y}\right)+g\left(y, Q^{2}\right) P_{g g}\left(\alpha_{s}\left(Q^{2}\right), \frac{x}{y}\right)\right)
\end{gathered}
$$

where the sum $i=1, \ldots, 2 n_{f}$ runs over quarks and antiquarks of all flavors. The functions $P_{a b}\left(\alpha_{s}\left(Q^{2}\right), z\right)$ are called splitting functions and represent the probability to find a parton $a$ in a parton $b$ at the scale $Q^{2}$ with a momentum fraction $z$. The splitting functions are calculable using a perturbative expansion in $\alpha_{s}$. The diagrams contributing to the leading order splitting functions are shown in figure 3.4.

In practice the PDFs used for calculations in the LHC energy regime are obtained by evolving the PDFs measured in fixed target experiments and in electron-proton scattering at HERA. The standard procedure is to first parametrize the $x$ dependence of the PDFs at a fixed input scale $Q_{0}^{2}$ and then extrapolate the function to the desired scale $Q^{2}$ according to the DGLAP equations. Several groups have performed PDF fits to the data obtained in DIS experiments, for example the CTEQ [38], MRST [39], MSTW [40] and NNPDF [41] groups. The quark and gluon distribution functions measured at HERA at $Q^{2}=10 \mathrm{GeV}$ are shown in figure 3.5 . 

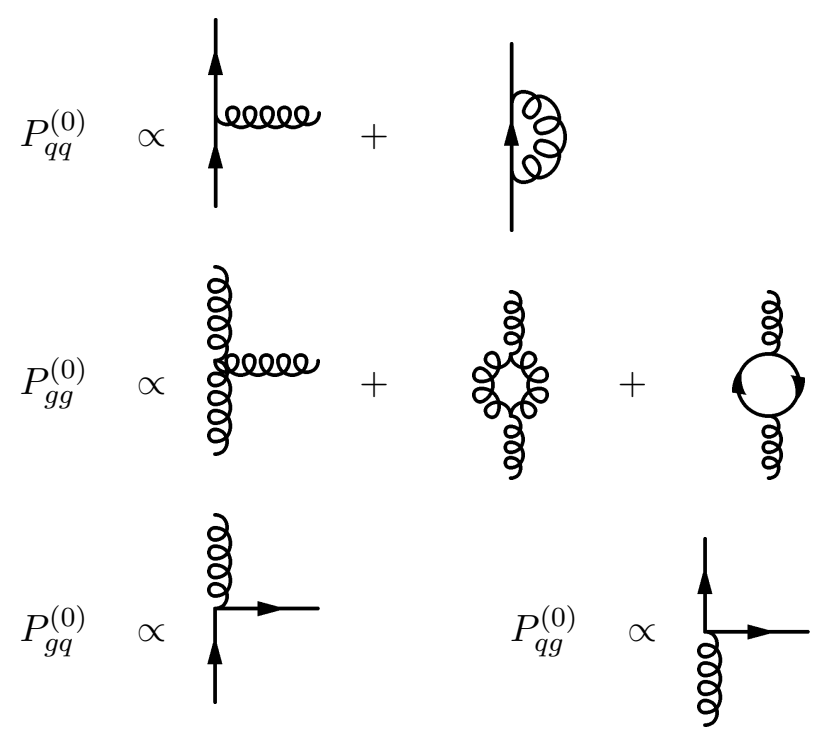

Figure 3.4: Feynman diagrams contributing to the leading order splitting functions.

\subsection{Heavy Quark Production}

The leading-order (LO) process for the production of a heavy quark $Q$ with mass $m_{Q}$ in hadronic collisions is flavor creation, i.e. quark-antiquark annihilation and gluon-gluon fusion

$$
q \bar{q} \rightarrow Q \bar{Q} \text { and } g g \rightarrow Q \bar{Q} .
$$

The corresponding diagrams are shown in figure 3.6. When evaluating these diagrams and integrating over the two-body phase space the total partonic cross section at LO in perturbation theory can be obtained [43]. The large energy limit of the partonic cross section is

$$
\begin{gathered}
\hat{\sigma}(q \bar{q} \rightarrow Q \bar{Q}) \rightarrow \frac{1}{\hat{s}} \\
\hat{\sigma}(g g \rightarrow Q \bar{Q}) \rightarrow \frac{1}{\hat{s}}\left(\frac{1}{\beta} \log \left(\frac{1+\beta}{1-\beta}\right)-2\right)
\end{gathered}
$$

where $\hat{s}$ is the center-of-mass energy available in the partonic system and $\beta \equiv \sqrt{1-\frac{4 m_{Q}^{2}}{\hat{s}}}$ is the velocity of the heavy quark. The quark annihilation process vanishes more quickly at high $\hat{s}$ thus gluon-gluon fusion is the dominant process for heavy quark production at the LHC. In flavor creation processes, the final states involving the heavy quarks are observed back-to-back with little combined transverse momentum.

At next-to-leading order (NLO), contributions of real and virtual emission diagrams have to be taken into account. In addition, heavy quarks can be produced in flavor excitation processes and gluon splitting events (figure 3.7). In the flavor excitation process, the heavy quark is considered to be already present in the incoming hadron. It is excited by the exchange of a gluon with the other hadron and appears on mass-shell in the final state. Since the heavy quark is not a valence quark it must originate from a pair production process $g \rightarrow Q \bar{Q}$. In most 


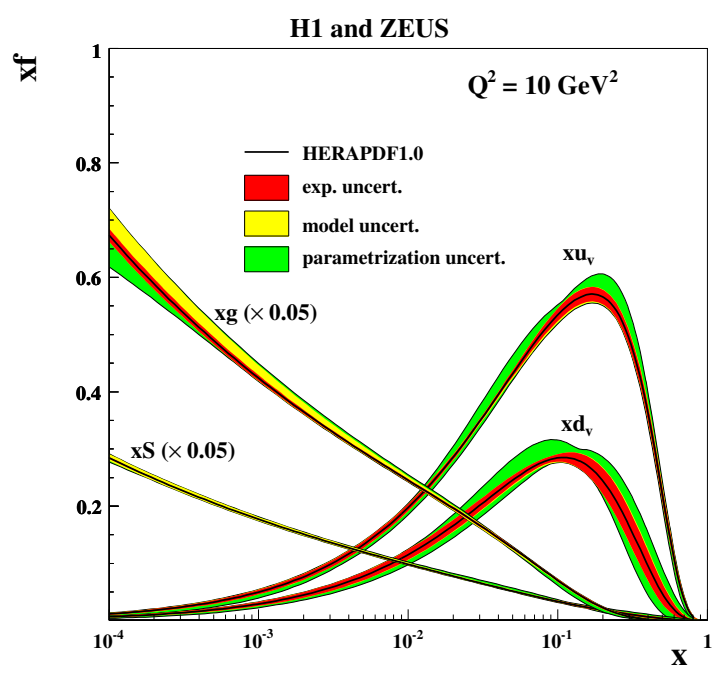

Figure 3.5: The proton parton distribution functions measured at HERA at $Q^{2}=10 \mathrm{GeV}$, for valence quarks $x u_{v}$ and $x d_{v}$, sea quarks $x S$, and gluons $x g$. The gluon and sea distributions are scaled down by a factor 20 [42].

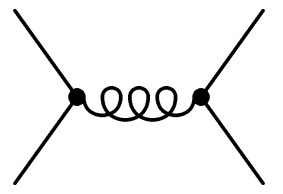

(a)

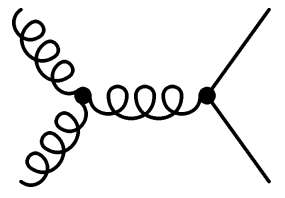

(b)

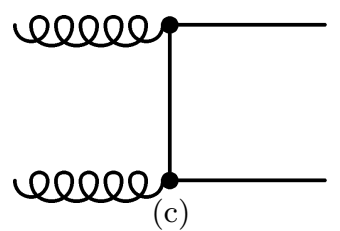

(c)

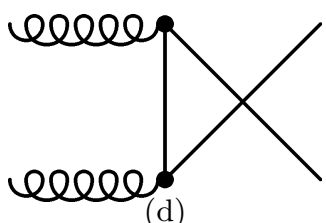

(d)

Figure 3.6: Leading order diagrams for heavy-quark pair production: (a) quark-antiquark annihilation $q \bar{q} \rightarrow Q \bar{Q}$, (b)-(d) gluon-gluon fusion $g g \rightarrow Q \bar{Q}$.

PDF parametrizations the heavy-flavor contributions are assumed to vanish for $Q^{2}<m_{Q}^{2}$, the hard scattering in flavor excitation processes must therefore have a virtuality above $m_{Q}^{2}$. The heavy quark final states do not need to be back-to-back as the third parton can carry away some transverse momentum.

In gluon splitting events the heavy quark occurs in $g \rightarrow Q \bar{Q}$ events in the initial- or finalstate shower. The resulting heavy flavored final state can carry a large combined transverse momentum and thus be concentrated within a small cone of angular separation. The contribution of the different processes to the total $b$-quark production cross section predicted by PYTHIA (see section 3.6) is shown in figure 3.8 as a function of the center of mass energy.

\subsection{The Fragmentation of Heavy Quarks}

The heavy quarks produced in the hard interaction are not visible in the detector due to color confinement. Instead the quarks fragment into color-singlet hadrons which then conglomerate in a collimated particle jet. As discussed in section 3.1, the fragmentation process happens on a larger time scale compared to the hard process and thus can be treated independently. 


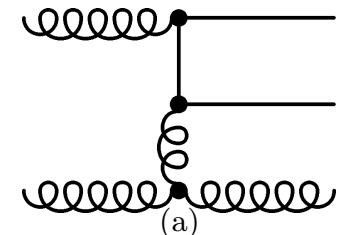

(a)

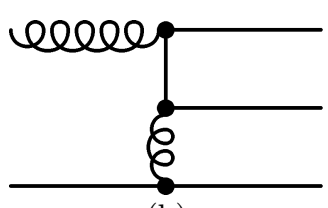

(b)

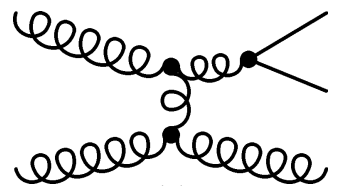

(c)

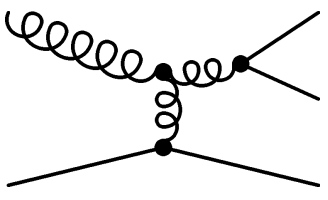

(d)

Figure 3.7: Next-to-leading order diagrams for heavy-quark pair production: (a),(b) flavor excitation, (c),(d) gluon splitting.

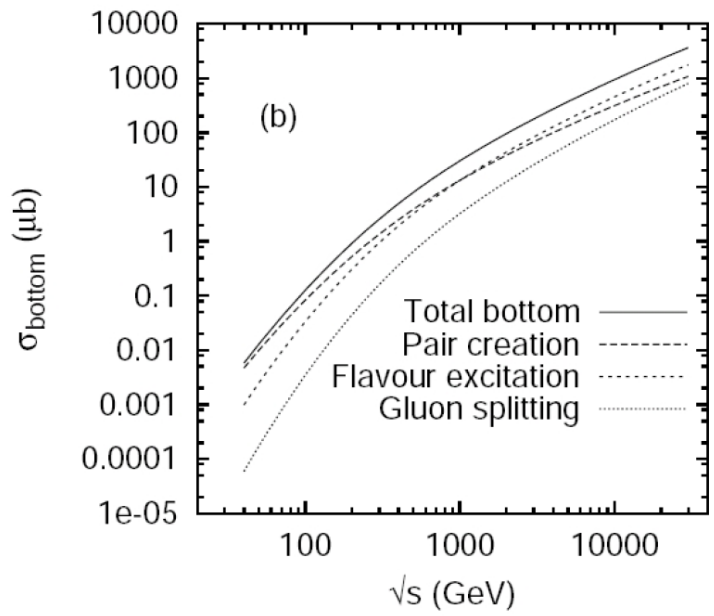

Figure 3.8: Total $b$ cross-section as a function of the center-of-mass energy $\sqrt{s}$ in proton-proton collisions. The contributions from pair production, flavor excitation and gluon splitting are shown [44].

Since the value of the coupling $\alpha_{s}$ rises strongly at large distances, the fragmentation process cannot be calculated from first principles in pQCD and phenomenological models have to be applied.

The probability to produce a hadron $h$ from a heavy quark $Q$ can be split in a short- and a long-range part [45]:

$$
\mathcal{D}_{Q}^{h}\left(z, \mu_{F}\right)=\int_{z}^{1} \mathcal{D}_{Q}\left(x, \mu_{F}\right) \mathcal{D}_{Q}^{h}\left(\frac{z}{x}\right) d x
$$

The short-distance, perturbative part $D_{Q}\left(x, \mu_{F}\right)$ models the evolution of a quark produced off-shell at the scale $\mu_{F}$ via gluon emissions to a quark on its mass shell. This is what is usually implemented in the parton shower algorithms of the Monte Carlo simulation programs. A parton shower develops through successive splitting until the perturbative approach becomes unreliable $\left(\sim \Lambda_{Q C D}\right)$. The parton shower represents an approximative perturbative treatment of QCD dynamics based on the DGLAP evolution equations. It improves the fixed order pQCD calculation by taking into account soft and collinear enhanced terms to all orders.

The set of partons in the low-momentum-transfer, long-distance regime produced in the parton shower is transformed into hadrons with the aid of phenomenological fragmentation 
functions $\mathcal{D}_{Q}^{h}(z)$. At present different models of the hadronization process exist among which the string and cluster fragmentation models are of interest for the analysis presented here. Under the assumption of factorization, the fragmentation functions do not depend on the hard scattering process. Hence, the fragmentation functions are universal and the models tuned for $e^{+} e^{-}$and $e p$ collision data can also be applied to the LHC data.

The string fragmentation model assumes linear confinement. In a physical picture, a color flux tube stretches between the $q \bar{q}$ pair as it starts to move apart. The potential energy stored in the string increases and the string may break by the production of a new quark pair $q_{1} \bar{q}_{1}$, so that the system splits into two color-singlet systems $q \bar{q}_{1}$ and $q_{1} \bar{q}$. If the invariant mass of either of these string pieces is large enough, further breaking might occur. Gluons are supposed to produce kinks on the strings which modifies the angular distribution of the hadrons inside the jet. The fragmentation function $\mathcal{D}_{Q}^{h}(z)$ determines the energy and the longitudinal momentum of the hadrons.

The most widely used formula for modeling the fragmentation of heavy quarks is the Peterson fragmentation function. The probability that the hadron receives a momentum fraction $z$ from the quark is given by [46]

$$
D_{Q}^{h}(z) \propto \frac{1}{z\left(1-\frac{1}{z}-\frac{\epsilon_{Q}}{1-z}\right)^{2}},
$$

where $\epsilon_{Q}$ is a free parameter that has to be measured in experiments. It is expected to scale between flavors like $\epsilon_{Q} \propto 1 / m_{Q}^{2}$. The values of the Peterson parameter for charm and beauty quarks extracted from electron-positron collision data are listed in table 3.1. The harder fragmentation of $b$ quarks is explained by their large mass. When binding a light quark to the heavy $b$ quark, the resulting hadron decelerates only slightly so that the $b$ quark and the hadron have almost the same momentum.

Table 3.1: Peterson parameter for charm and beauty quarks extracted from electron-positron collision data at a center of mass energy $\sqrt{s}$. The parameters have been determined at LO and NLO [45].

\begin{tabular}{cccc}
\hline & $\sqrt{s}[\mathrm{GeV}]$ & $\mathrm{LO}\left(\alpha_{s}\right)$ & $\mathrm{NLO}\left(\alpha_{s}\right)$ \\
\hline$\epsilon_{c}$ & 10.5 & 0.058 & 0.035 \\
$\epsilon_{c}$ & 91.2 & 0.078 & 0.040 \\
\hline$\epsilon_{b}$ & 91.2 & 0.0069 & 0.0033 \\
\hline
\end{tabular}

In case of cluster fragmentation models color-singlet clusters of partons form after the perturbative phase of jet development and then decay into the observed hadrons. The clusters originate from gluon splitting in quark pairs and subsequent recombination with neighboring quarks and antiquarks. Afterward, the clusters are assumed to decay isotropically in their rest frame into pairs of hadrons, where the branching ratios are determined by the density of states.

\subsection{Semileptonic Decays of Heavy Quarks}

The presence of hadrons containing heavy quarks is deduced by the observation of their decay products. In a first approximation of $b$-flavored hadron decays, only the beauty quark 
participates in the transition while the other quark acts as a spectator quark. The $b$ quark can decay via the weak interaction into a $c$ - or a $u$-quark. The charged current couplings for the flavor-changing transition between quarks are described in terms of the Cabbibo-KobayashiMaskawa (CKM) [47, 48] matrix given by

$$
V_{\mathrm{CKM}}=\left(\begin{array}{ccc}
V_{u d} & V_{u s} & V_{u b} \\
V_{c d} & V_{c s} & V_{c b} \\
V_{t d} & V_{t s} & V_{t b}
\end{array}\right) .
$$

The universality of the weak decay is reflected in the unitarity of the CKM matrix. Hence, the CKM matrix can be parametrized by three mixing angles and one irreducible phase which accounts for the $C P$-violation intrinsic to the weak decay in the Standard Model. The decay width is proportional to the squared CKM matrix element. Measurements of semileptonic decays of $B$ mesons have shown that the matrix elements relevant for the weak decay of the $b$ quark are very small compared to other elements: $\left|V_{c b}\right|=0.0412 \pm 0.0011$ and $\left|V_{u b}\right|=0.00393 \pm 0.00036$ [49]. Consequently, the $b$ quark decay is highly suppressed and the $b$ quark has a relatively large lifetime of $\tau \sim 10^{-12} \mathrm{~s}$. Since $\left|V_{c b}\right|$ is about an order of magnitude larger than $\left|V_{u b}\right|$ the preferred decay is $b \rightarrow c W^{-}$with a branching ratio of almost $100 \%$.

The lifetime $\tau$ of a $b$-hadron is related to the decay length $l$ by

$$
l=\beta \gamma c \tau=\frac{p_{B}}{m_{B}} c \tau,
$$

where $p_{B}, m_{B}$ and $\beta \gamma$ are the particle's momentum, mass and boost, respectively. The mean decay length of beauty hadrons is $c \tau=466 \mu \mathrm{m}$ (see also table 3.2). This transforms into an average observable decay length of $\beta \gamma c \tau=3-5 \mathrm{~mm}$ in the rest frame at the LHC which can be observed as a displaced (or secondary) vertex in the detector. Objects originating from a secondary vertex are generally characterized by a large transverse impact parameter (figure 3.9) and can thereby be identified. In CMS a lifetime based tag of $b$-hadrons is possible thanks to the pixel detector which achieves a track impact parameter resolution of $\sigma=80 \mu \mathrm{m}$ for $p_{T}>7 \mathrm{GeV}$ and $\sigma=90 \mu \mathrm{m}$ for $p_{T}=4 \mathrm{GeV}[50]$.

Table 3.2: Properties of $b$-hadrons. The table shows the quark content, the mass, the lifetime and the decay length [49].

\begin{tabular}{ccccc}
\hline & quark content & mass & lifetime & decay length \\
& & $m[\mathrm{MeV}]$ & $\tau[\mathrm{ps}]$ & $c \tau[\mu \mathrm{m}]$ \\
\hline$B^{+}$ & $u \bar{b}$ & $5279.17 \pm 0.29$ & $1.638 \pm 0.011$ & 491.1 \\
$B^{0}$ & $d \bar{b}$ & $5279.50 \pm 0.30$ & $1.525 \pm 0.009$ & 458.7 \\
$B_{s}^{0}$ & $s \bar{b}$ & $5366.3 \pm 0.6$ & $1.425 \pm 0.041$ & 441 \\
$\Lambda_{b}^{0}$ & $u b d$ & $5620.2 \pm 1.6$ & $1.383_{-0.048}^{+0.049}$ & 415 \\
\hline
\end{tabular}

The $W$ boson originating from the weak decay of the $b$ quark decays either hadronically or leptonically. Within this analysis the semileptonic decay of $b$ quarks into muons is studied since the muon provides a clean signature which is relatively easy to detect experimentally. The decay $W^{-} \rightarrow \mu^{-} \bar{\nu}_{\mu}$ has a branching ratio of about $10 \%$. In addition, about $10 \%$ of the subsequent charm decays also have a muon and a neutrino in the final state. The Feynman diagrams of the semileptonic decay of a $b$-hadron with a muon in the final state are illustrated in figure 3.10 . 


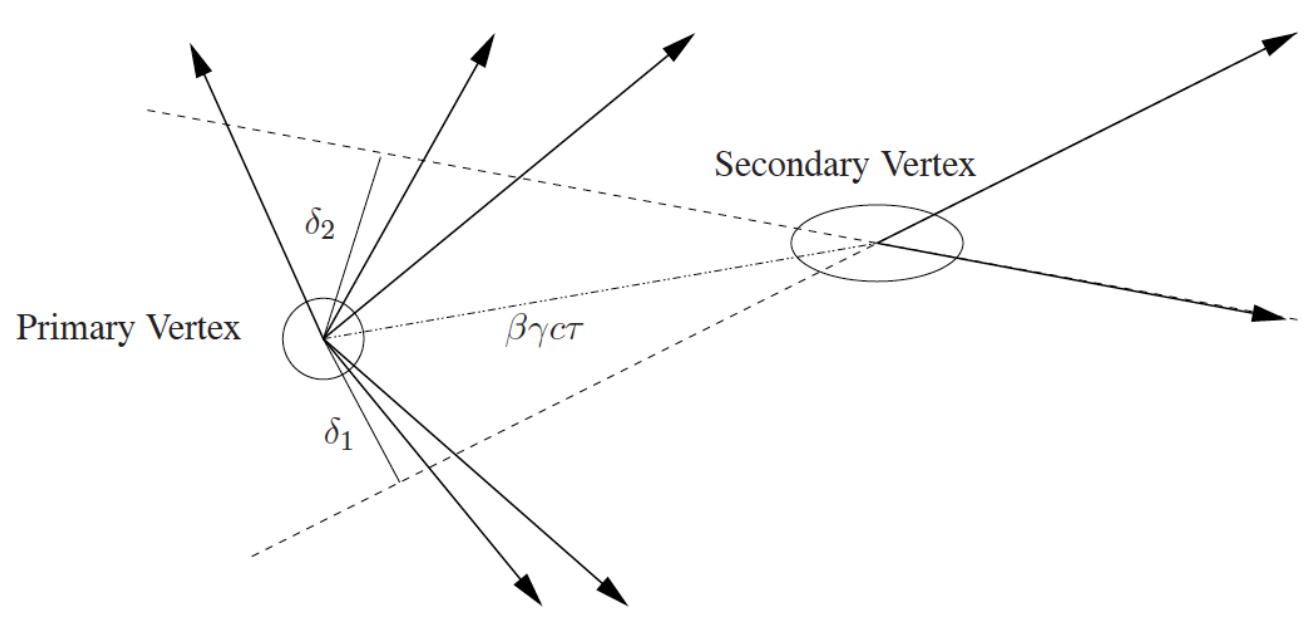

Figure 3.9: Illustration of the transverse impact parameter $\left(\delta_{1}, \delta_{2}\right)$ of the decay products of a long-lived particle. The decay particles emerging from the secondary vertex are characterized by a large impact parameter compared to the impact parameters of the particles emerging from the primary vertex (figure from [51]).

\subsection{Monte Carlo Event Generators}

Monte Carlo (MC) event generators provide an event-by-event prediction of complete hadronic final states based on QCD calculation. They allow to study the topology of events generated in hadronic interactions and are used as input for detector simulation programs to investigate detector effects. The event simulation is divided into different stages as illustrated in figure 3.11. First, the partonic cross section is evaluated by calculating the matrix element in fixed order pQCD. The event generators presently available for the simulation of protonproton collisions provide perturbative calculations for beauty production up to NLO. Higher order corrections due to initial and final state radiation are approximated by running a parton shower algorithm. The parton shower generates a set of secondary partons originating from subsequent gluon emission of the initial partons. It is followed by the hadronization algorithm

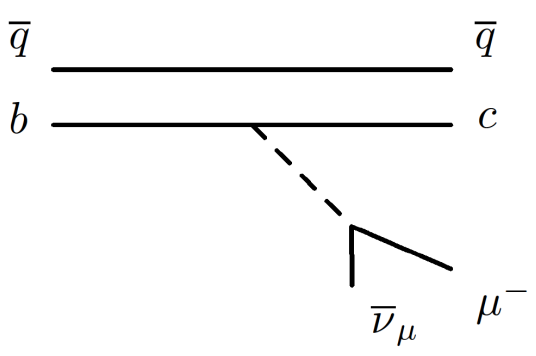

(a)

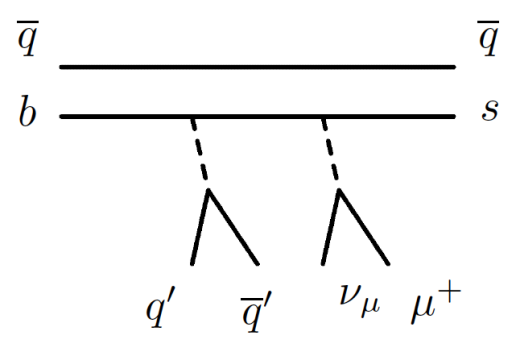

(b)

Figure 3.10: Semileptonic weak decay of $b$-hadrons with a muon in the final state. 
which clusters the individual partons into color-singlet hadrons. In a final step, the short lived hadrons are decayed.

In the framework of the analysis presented here, the MC event generators PYTHIA $6.4[52,53,54]$, HERWIG 6.5.10 [55, 56, 57], and MC@NLO $3.4[58,59]$ are used to compute efficiencies, kinematic distributions, and for comparisons with the experimental results. All programs were run with their default parameter settings, except when mentioned otherwise.

\section{PYTHIA}

In the PYTHIA program, the matrix elements are calculated in LO pQCD and convoluted with the proton PDF, chosen herein to be CTEQ6L1. The mass of the $b$-quark is set to $m_{b}=4.8 \mathrm{GeV}$. The underlying event is simulated with the D6T tune [93]. Pile-up events were not included in the simulation. The parton shower algorithm is based on a leading-logarithmic approximation for QCD radiation and a string fragmentation model (implemented in JETSET [61, 62]) is applied. The longitudinal fragmentation is described by the Lund symmetric fragmentation function [63] for light quarks and by the Peterson fragmentation function for charm and beauty quarks. The parameters of the Peterson fragmentation function are set to $\epsilon_{c}=0.05$ and $\epsilon_{b}=0.005$. In order to estimate the systematic uncertainty introduced by the choice of the fragmentation function, samples generated with different values of $\epsilon_{b}$ are studied

The hadronic decay chain used in PYTHIA is also implemented by the JETSET program. For comparison, additional event samples are generated where the EvtGen [64] program is used to decay the $b$-hadrons. EvtGen is an event generator designed for the simulation of the physics of $b$-hadron decays, and in particular provides a framework to handle complex sequential decays and $C P$ violating decays.

\section{HERWIG}

The HERWIG program incorporates color coherence effects in the final state and initial state parton showers, as well as in heavy quark processes and the hard process generation. HERWIG uses a low-mass cluster hadronization model. Multiparton interactions and the underlying event are simulated by the JIMMY package [65]. The value of the $b$-quark mass is set to $m_{b}=4.8 \mathrm{GeV}$ and the CTEQ6L1 PDF sets are used.

\section{$\mathrm{MC} @ \mathrm{NLO}$}

The MC@NLO package has a NLO matrix element calculation interfaced to the parton shower algorithms of the HERWIG package. The mass of the b-quark is set to $m_{b}=4.75 \mathrm{GeV}$. The CTEQ6M PDF sets were used to generate the MC@NLO events. The events generated with MC@NLO are studied only at the generator level and are not passed through the detailed detector simulation. 


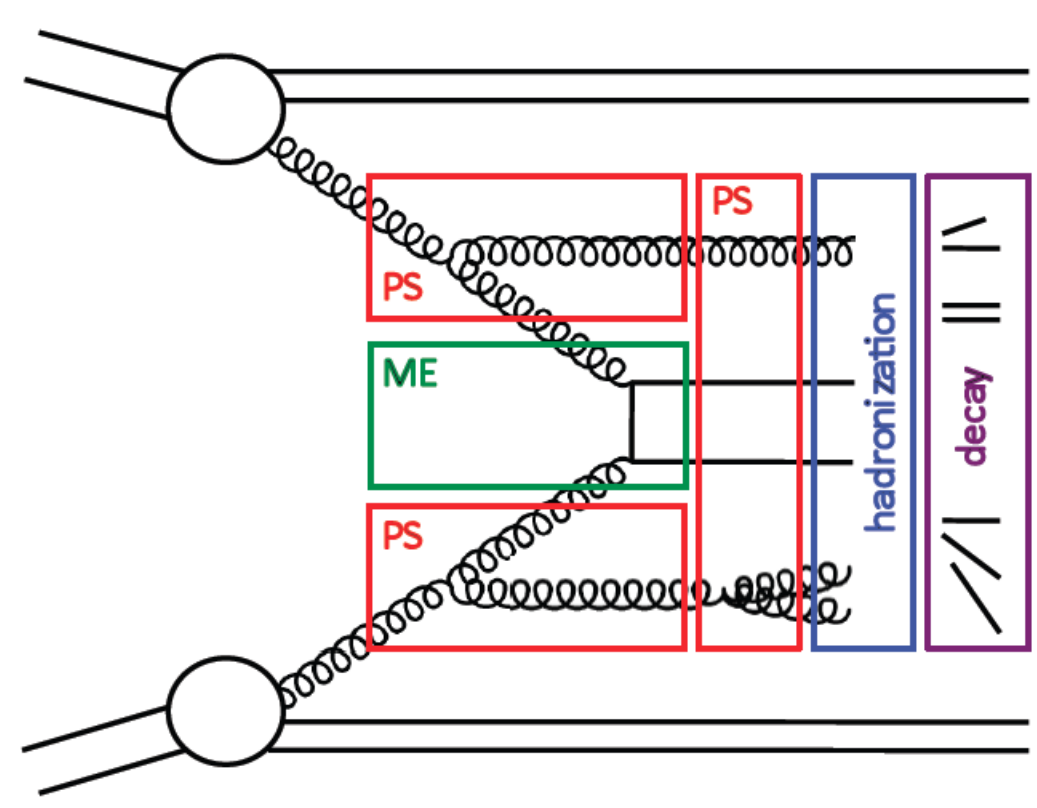

Figure 3.11: Schematic view of the subsequent steps of a MC event generator: matrix element $(\mathrm{ME})$, parton shower (PS), hadronization and decay. 



\section{Chapter 4}

\section{Study of the Inclusive Beauty Production}

The prospects for a measurement of the inclusive $b$-quark production cross-section with the very early data at CMS are presented in this chapter. Due to the large $b$-quark production cross-section, high statistics data samples are expected soon after the LHC start-up. Measurements of the $b$-quark production have already been done at the Tevatron $[8,66,9,67]$, HERA $[68,69,70,71]$ and other colliders. A lot of progress has been made in understanding the $b$-quark production process and the measurements are in reasonable agreement with NLO/NLL QCD predictions in most regions of the phase space. However, theoretical uncertainties are sizeable and there is a great interest in verifying the results at the higher centerof-mass energy provided by the LHC. The investigation of $b$-quark production is interesting on its own, but also because events containing $b$ quarks present an important background to most of the searches at the LHC. In the first section the concept of the measurement is introduced, followed by an overview of the event samples used in the analysis. Thereafter the trigger and offline selection are discussed. The fitting procedure to determine the fraction of signal events among the selected events is described and data-driven methods for validating the MC templates used in the fit are presented. Furthermore, the method for extracting the inclusive and the differential $b$-quark production cross-section is reviewed. The chapter closes with a discussion of the main systematic uncertainties and the results.

\subsection{Concept}

In the context of this analysis the semileptonic decay of $b$ quarks into muons and jets is studied. The muons provide a clean signal in the detector which permits to identify them already on trigger level. Muons from $b$ - and $c$-quark decays can be distinguished by their momentum distribution. Due to the higher mass of the $b$ quark $\left(m_{b}=4.95 \mathrm{GeV}\right)$ more energy is transferred to the daughter particle on average. Furthermore, the transverse momentum of the muon relative to the fragmentation jet $\left(p_{\perp}^{\text {rel }}\right)$ is larger in $b$-events than in $c$ - and light quark events. The $p_{\perp}^{\text {rel }}$ variable is illustrated in figure 4.1 and will be used in this analysis to discriminate signal events from background events.

At a center-of-mass energy of $\sqrt{s}=7 \mathrm{TeV}$, an inclusive $b$-quark production cross-section of $\sigma_{b}=322 \mu \mathrm{b}$ is predicted by PYTHIA. The simulated transverse momentum and pseudorapidity distributions of the $b$ quark are shown in figure 4.2 . In about $20 \%$ of the cases a 


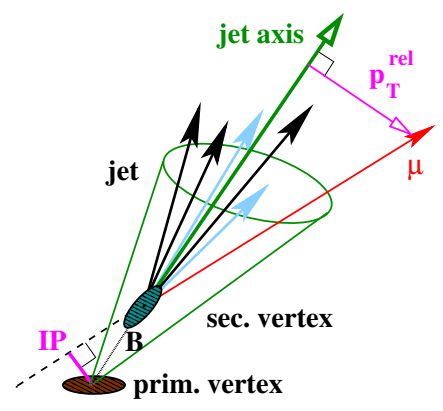

Figure 4.1: Illustration of the $p_{\perp}^{\mathrm{rel}}$ variable which denotes the component of the muon momentum perpendicular to the jet direction.

muon is found among the decay products of the $b$ quark. In figure 4.3 the transverse momentum and the pseudorapidity of the muon generated either in the semileptonic decay of the $b$ quark or the subsequent $c$ quark is displayed. The visible kinematic range for muons in the measurement presented here is $p_{T}>5 \mathrm{GeV}$ and $-2.1<\eta<2.1$, which corresponds to an acceptance of $2 \%$.

The $p_{\perp}^{\text {rel }}$ variable is defined with respect to the axis of the fragmentation jet which is reconstructed from charged particle tracks only. The tracks are clustered by a jet algorithm (TrackJets) and a combined transverse energy of $E_{T}>1 \mathrm{GeV}$ is required. In order to facilitate the comparison between the measurement and the theory predictions, the TrackJet is not included in the cross section definition. The extrapolation of the measured cross-section amounts to $10 \%$.

\subsection{Event Simulation}

The MC event samples were generated, simulated and reconstructed within the official CMS software framework (CMSSW) versions CMSSW_2_2_X and CMSSW_3_1_X. The generation of Monte Carlo event samples is based on PYTHIA V6 and does not include pile-up events. All QCD signal and background events are selected from the generic $2 \rightarrow 2$ subprocesses (default PYTHIA MSEL=1 card) and present a mixture of gluon-gluon fusion, flavor excitation, and gluon splitting events. No attempt is made to separate the production mechanisms and all events containing a $b$ quark are counted as $b$-events. Events containing a $c$ quark but no $b$ quark are called $c$-events. All remaining events are called $u d s g$-events.

The samples were simulated in separate $\hat{p}_{T}$ ranges and event weights are introduced to scale the contribution of the individual $\hat{p}_{T}$ bins according to the cross section predicted by PYTHIA.

A muon filter is applied to all generated events. A special procedure has been used to efficiently simulate inclusive muon samples including prompt muons and muon originating from in-flight decays of pions and kaons. An inclusive muon sample is achieved by forcing kaons and pions to decay already during generation rather than during simulation. The generated muon has to be in the kinematic range of $-2.5<\eta<2.5$ and $p_{T}>5 \mathrm{GeV}$, except for the two QCD samples with $\hat{p}_{T}>0 \mathrm{GeV}$ where it is lowered to $p_{T}>2.5 \mathrm{GeV}$. For signal 

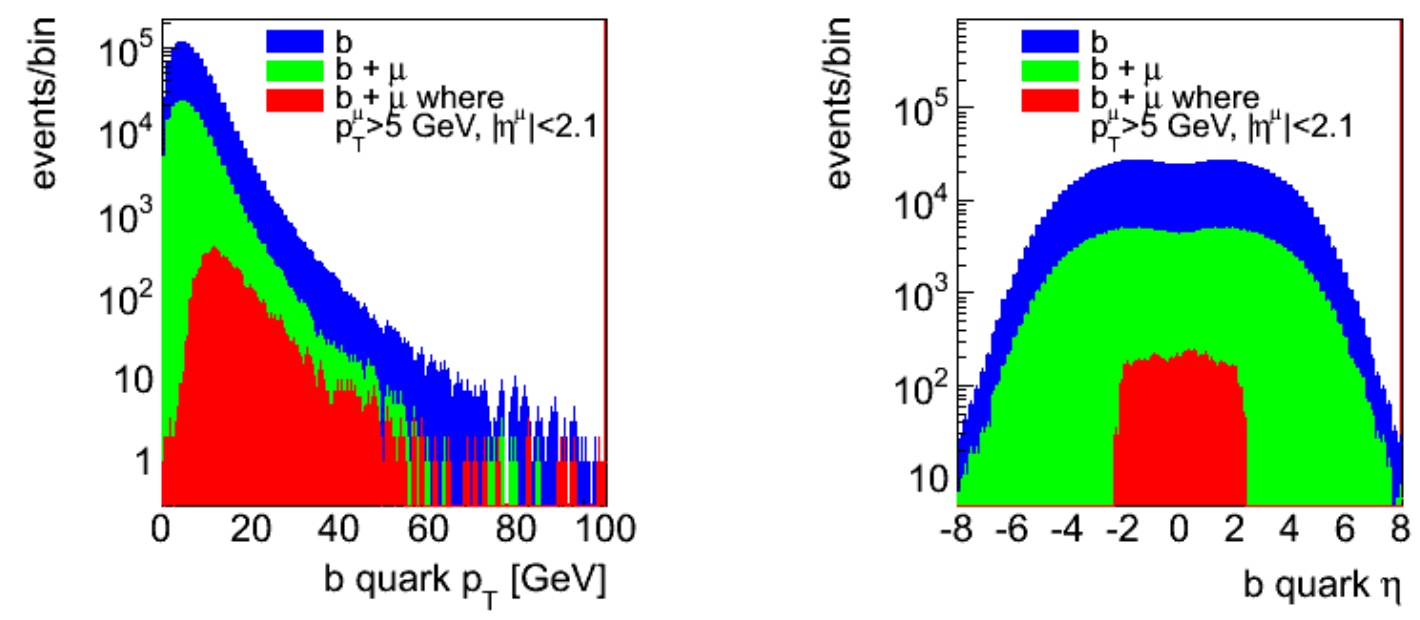

Figure 4.2: Transverse momentum (left) and pseudorapidity (right) distribution of $b$ quarks originating from proton-proton collisions at a center-of-mass energy of $\sqrt{s}=7 \mathrm{TeV}$. The inclusive distribution is shown in blue, the green distribution corresponds to $b$ quarks that decay semileptonically into muons and the red one describes quarks whose decay produce muons within the visible kinematic range $\left(p_{T}>5 \mathrm{GeV}\right.$ and $\left.-2.1<\eta<2.1\right)$.
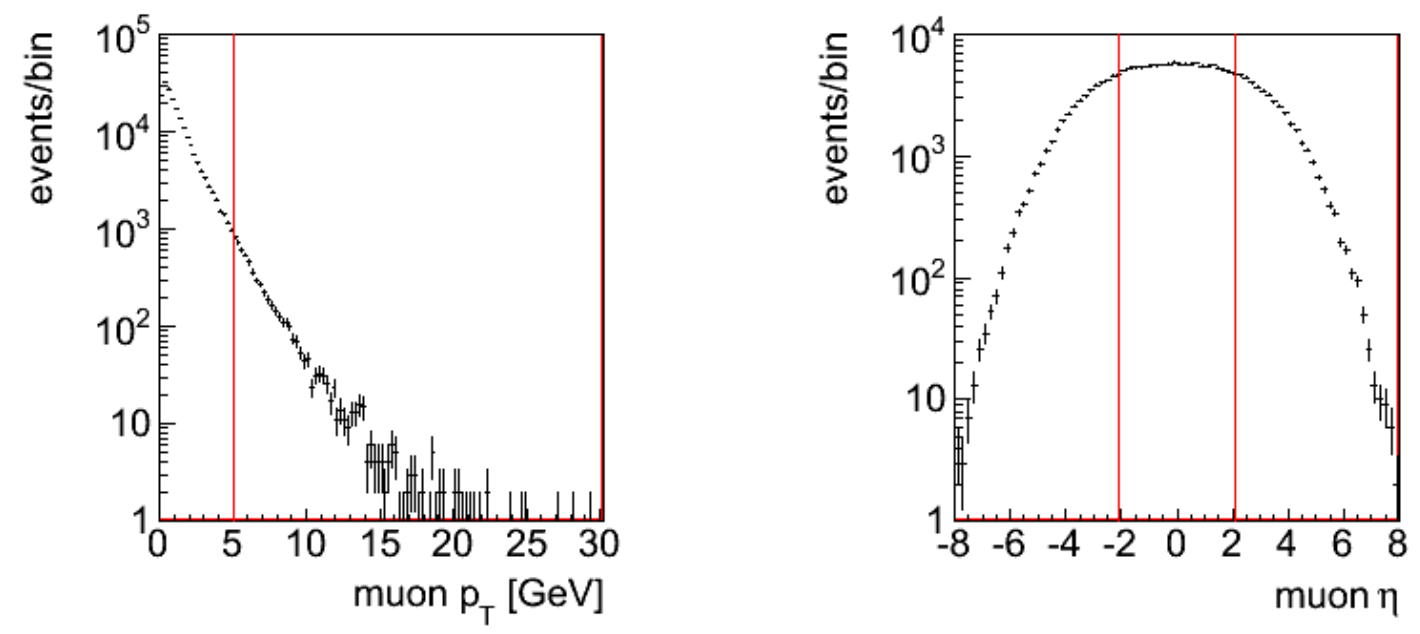

Figure 4.3: Generated transverse momentum (left) and pseudorapidity (right) distribution of muons produced in the semileptonic decay of $b$ quarks. The red lines at $p_{T}=5 \mathrm{GeV}$ and $|\eta|=2.1$ indicate the visible kinematic range.

events, an additional filter is applied requiring a $b$ quark in the generated event.

The events undergo a full detector simulation based on GEANT4 [72] including a emula- 
tion of the first level trigger. Subsequent high level triggers and offline reconstruction use the same code as applied to data.

Table 4.1 provides a summary of the event samples used in the analysis. If not stated otherwise the MC simulation corresponding to a center-of-mass energy of $\sqrt{s}=7 \mathrm{TeV}$ is used to obtain the results in this chapter.

Table 4.1: Overview of the event samples used in the analysis. In the first three columns, the center-of-mass energy of the proton-proton collision, the cross section (PYTHIA MSEL=1 card) and the integrated luminosity of the samples are given. The remaining columns state the filter efficiency of the generator level filter, the number of events that pass the filter and the fraction of $b$-events among the filtered events. The transverse momentum threshold for the generated muon is $p_{T}>5 \mathrm{GeV}$, except for the two QCD samples with $\hat{p}_{T}>0 \mathrm{GeV}$ where it is lowered to $p_{T}>2.5 \mathrm{GeV}$.

\begin{tabular}{lrrrrrr}
\hline Sample & $\sqrt{s}[\mathrm{TeV}]$ & $\sigma[\mu \mathrm{b}]$ & $\mathcal{L}\left[\mathrm{pb}^{-1}\right]$ & $\epsilon_{\text {filt }}$ & $N_{\text {filt }}$ & $N_{\text {filt }}^{b} / N_{\text {filt }}$ \\
\hline Signal: & & & & & & \\
$\hat{p}_{T}>20 \mathrm{GeV}$ & 10 & 497.2 & 2.9 & 0.0037 & 5292356 & 1 \\
QCD (inclusive): & & & & & & \\
$\hat{p}_{T}>0 \mathrm{GeV}$ & 10 & 51560 & 0.04 & 0.0023 & 4844040 & 0.20 \\
$\hat{p}_{T}>20 \mathrm{GeV}$ & 10 & 497.2 & 2.4 & 0.0079 & 9623100 & 0.47 \\
$30<\hat{p}_{T}<50 \mathrm{GeV}$ & 10 & 91.78 & 9.2 & 0.01 & 8411460 & 0.46 \\
$50<\hat{p}_{T}<80 \mathrm{GeV}$ & 10 & 11.66 & 10.3 & 0.021 & 2511780 & 0.44 \\
$80<\hat{p}_{T}<120 \mathrm{GeV}$ & 10 & 1.55 & 14.4 & 0.033 & 735244 & 0.43 \\
$120<\hat{p}_{T}<170 \mathrm{GeV}$ & 10 & 0.25 & 30.0 & 0.044 & 328590 & 0.42 \\
$\hat{p}_{T}>170 \mathrm{GeV}$ & 10 & 0.06 & 125.3 & 0.06 & 466279 & 0.40 \\
$\hat{p}_{T}>0 \mathrm{GeV}$ & 7 & 48440 & 0.12 & 0.0018 & 10314205 & 0.21 \\
\hline
\end{tabular}

\subsection{Trigger}

The events of interest are selected by requiring at least one single muon on trigger level. The muon identification on trigger level is based on subsequent reconstruction and filtering steps. As discussed in section 2.2.9, the decision of the L1 muon trigger is based on the information of the muon system only. The measurement of the muon system and the tracking detectors are combined at the HLT in case a muon candidate is present at L1. First a standalone (L2) muon reconstruction is performed using the parameters of the L1 muon candidate for seeding. If a standalone muon with transverse momentum above threshold is found, it serves in turn as a seed for the global muon (L3) reconstruction. After the reconstruction has terminated, further cuts are applied on the transverse momentum of the global muon and the track impact parameter in the transverse plane $\left(d_{0}<2 \mathrm{~cm}\right)$. An event is accepted on trigger level provided that a L3 muon passing the last filter module is present.

The performance of the muon trigger has been studied in detail in [19]. In the framework of this analysis two HLT paths (HLT_Mu3 and HLT_Mu5) which mainly differ in the transverse momentum threshold $\left(p_{T}>3(5) \mathrm{GeV}\right)$ are investigated. The L1 and HLT conditions for these paths are listed in table 4.2 .

The muon transverse momentum threshold on L1 and HLT have been optimized for the LHC startup assuming an instantaneous luminosity of $\mathrm{L}=8 \cdot 10^{29} \mathrm{~cm}^{-2} \mathrm{~s}^{-1}$. When the lumi- 
Table 4.2: Transverse momentum threshold (in $\mathrm{GeV}$ ) applied to the muon candidates on trigger level for the HLT paths investigated in this analysis.

\begin{tabular}{cccc}
\hline & L1 & L2 & L3 \\
\hline HLT_Mu3 & 0 & 3 & 3 \\
HLT_Mu5 & 3 & 4 & 5 \\
\hline
\end{tabular}

nosity delivered by the LHC increases, the single muon trigger will have a higher rate which at some point will exceed the allocated bandwidth. In this case either the transverse momentum threshold has to be increased or prescale factors have to be applied. Table 4.3 shows the muon trigger transverse momentum threshold values projected for increased values of the LHC luminosity.

Table 4.3: Expected HLT transverse momentum threshold for the single muon and di-muon trigger paths for different luminosities.

\begin{tabular}{lcc}
\hline Luminosity $\left[\mathrm{cm}^{-2} \mathrm{~s}^{-1}\right]$ & Single muon & Di-muon \\
\hline $8 \cdot 10^{29}$ & 3 & $3 / 3$ \\
$10^{31}$ & 5 & $3 / 3$ \\
$2 \cdot 10^{31}$ & 11 & $3 / 3$ \\
$10^{32}$ & 16 & $3 / 3$ \\
\hline
\end{tabular}

The trigger efficiency is determined by a trigger simulation. The combined L1 and HLT efficiency for signal events are displayed in figure 4.4 as a function of muon transverse momentum and pseudorapidity. The efficiency is calculated with respect to the number of generated particles in the acceptance of the measurement, i.e $|\eta|<2.1$ and $p_{T}>5 \mathrm{GeV}$. For HLT_Mu3 and HLT_Mu5 the efficiency plateau is reached for muons with $p_{T}>18-20 \mathrm{GeV}$ and lies around $90 \%$. The slightly lower efficiency of HLT_Mu5 is due to the more restrictive L1 condition. Besides the higher transverse momentum also a better quality (see section 2.2.9) of the muon candidate is required.

The overall trigger efficiency for $b \rightarrow \mu X$ events that have a muon with $p_{T}>5 \mathrm{GeV}$ in $-2.1<\eta<2.1$ on generator level is $84 \%$ for HLT_Mu3, while it is $79 \%$ for HLT_Mu5.

\subsection{Jet Reconstruction}

The use of a collinear and infrared safe jet reconstruction algorithm in an analysis is strongly recommended when comparing the results to the theoretical predictions. In this analysis jets are reconstructed by the anti- $k_{\mathrm{T}}$ algorithm [73]. The algorithm is based on successive pairwise recombination of particles according to the distance between any two particles $i$ and $j$ $\left(d_{i j}\right)$ and the distance of any particle $i$ to the beam $\left(d_{i B}\right)$ which are defined as

$$
\begin{gathered}
d_{i j}=\min \left(k_{\mathrm{T} i}^{-2}, k_{\mathrm{T} j}^{-2}\right) \frac{\left[\left(y_{i}-y_{j}\right)^{2}+\left(\phi_{i}-\phi_{j}\right)^{2}\right]}{D^{2}}, \\
d_{i B}=k_{\mathrm{T} i}^{-2},
\end{gathered}
$$



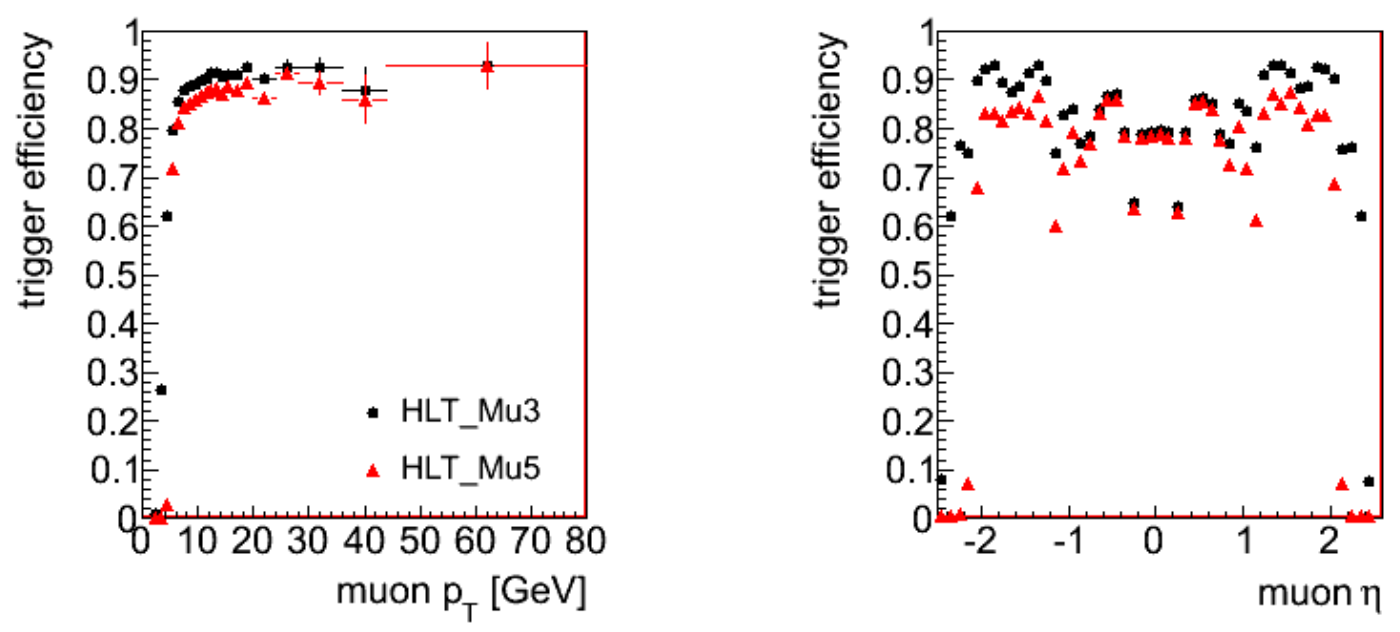

Figure 4.4: The single muon trigger efficiency as a function of the muon transverse momentum and pseudorapidity in signal events for HLT_Mu3 (black circles) and HLT_Mu5 (red triangles). In the left plots the efficiencies are determined with respect to generated muons with pseudorapidity $-2.1<\eta<2.1$, in the right plots for muons with transverse momentum $p_{T}>5 \mathrm{GeV}$.

where $k_{\mathrm{T} i}, y_{i}$ and $\phi_{i}$ are the transverse momentum, the rapidity and the azimuthal angle of particle $i$, respectively. Jets are defined in an iterative procedure. If the smallest distance is a $d_{i j}$, the corresponding particles are combined, otherwise particle $i$ is defined as a jet. The anti- $k_{\mathrm{T}}$ algorithm is reasonably fast and collinear and infrared safe to all orders in the perturbative expansion. The jet size parameter $D$ gives the minimum distance between two jets and a value of $D=0.5$ is chosen here.

Three kinds of jet objects are produced by the SISCone algorithm: calorimeter jets (CaloJets), tracker jets (TrackJets) [74] and generated particle jets (GenJets). The latter are clustered from all stable generated particles and are a measure of the hadron-level jet content. The reconstruction of CaloJets is based on energy depositions in calorimeter towers. A calorimeter tower is built from one HCAL cell and $5 \times 5$ ECAL crystals in the barrel, while a more complex association is required in the endcaps [75]. Three stages of jet corrections are applied offline to CaloJets [76]: offset corrections for pile-up and noise, correction of the calorimeter response as a function of pseudorapidity and correction for the absolute response as a function of transverse momentum.

TrackJets are obtained when using only charged particle tracks as input to the jet algorithm. They are expected to give a better result when reconstructing low transverse energy jets. This is due to the fact that low momentum charged particles might not reach the calorimeter or might be strongly deflected from the original direction. The use of TrackJets also provides an independent measurement with its own systematic uncertainty. It has the additional benefit of an improved jet angular resolution (mainly in $\phi$ ) as can be seen in figure 4.5. Here, the jet resolution in azimuthal angle and pseudorapidity is presented for events in which a $b$ quark decays semileptonically into a muon and a jet. A good understanding of 
the jet angular resolution and its uncertainty is crucial for the measurement as the relative transverse momentum of the muon with respect to the jet is computed to discriminate signal events from background events. The disadvantage of using jets reconstructed from tracks only is their low energy response (figure 4.6). The energy of a TrackJet will always be lower than the energy of the corresponding GenJet since the fraction of jet momenta carried by neutral particles is not visible in the tracking detectors.
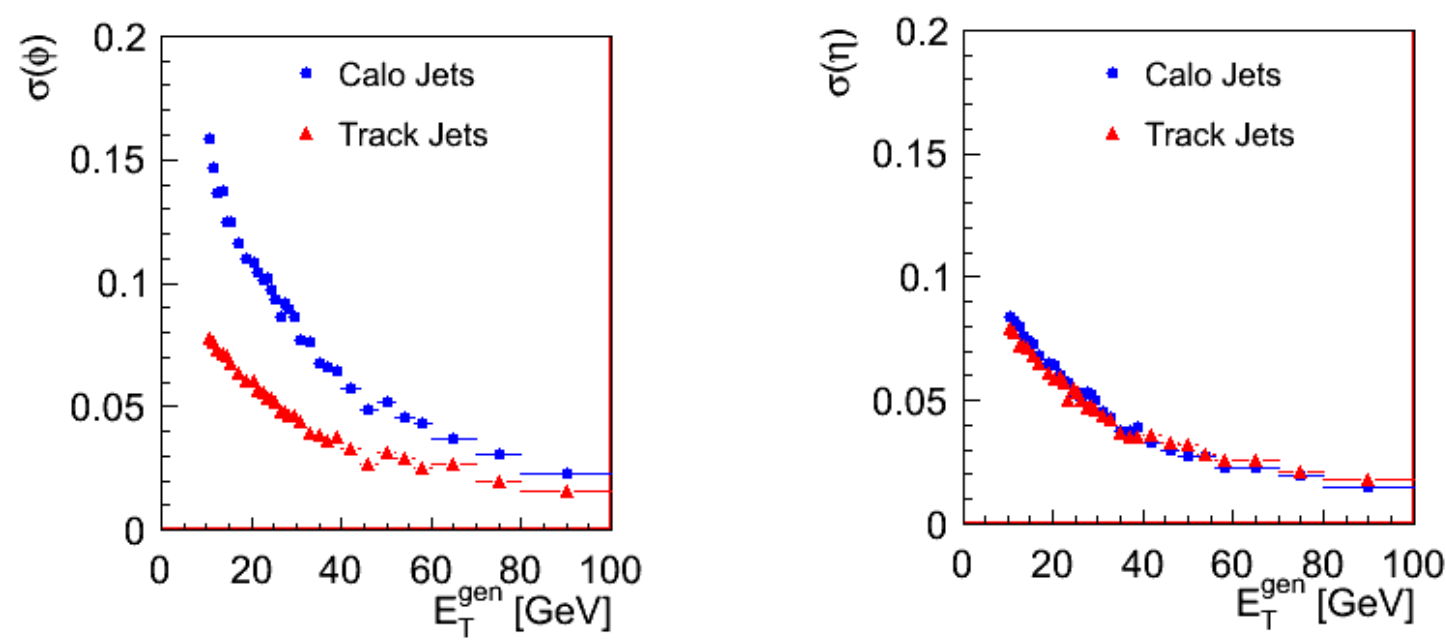

Figure 4.5: The jet angular resolution as a function of the transverse energy of the generated jet. The left plot shows the resolution in azimuthal angle, the right plot the resolution in pseudorapidity. The angular resolution of TrackJets (red triangles) is compared to the resolution of CaloJets (blue circles).

In most cases the muon emitted in the decay of a $b$ quark is reconstructed within the jet. Since the event selection developed for the analysis presented here starts from the muon reconstructed on trigger level, the jet containing the muon is identified as a $b$-jet. Figure 4.7 shows the transverse energy spectrum for $b$-jets reconstructed from CaloJets and TrackJets, respectively. The spectrum is falling steeply and a mean value of $E_{T}=5.7 \mathrm{GeV}$ for TrackJets and $E_{T}=12.2 \mathrm{GeV}$ for CaloJets is found. The transverse energy of the TrackJet is calculated by summing the four-momenta of its constituents which are assumed to be pions. The muon is not considered as a jet constituent, i.e. the muon track is excluded from the TrackJet. The three stages of jet corrections are applied to correct the response of the CaloJets back to the particle level jet, whereas the CaloJet is not corrected for the energy deposition of the muon in the calorimeter. The size of the correction depends on the transverse energy of the generated jet. According to [76] correction factors of the order of 2.5 to 3.2 have to be applied for jets with $E_{T}<10 \mathrm{GeV}$. These numbers are currently determined from simulations and affected by a significant systematic uncertainty. In order to avoid entering the phase space region in which large systematic uncertainties on the cross section are introduced due to the large jet energy correction factors, the CaloJets are required to have a minimum raw transverse energy of $E_{T}>10 \mathrm{GeV}$.

TrackJets prove to be more reliable when going to low jet energies. Within this analysis, 


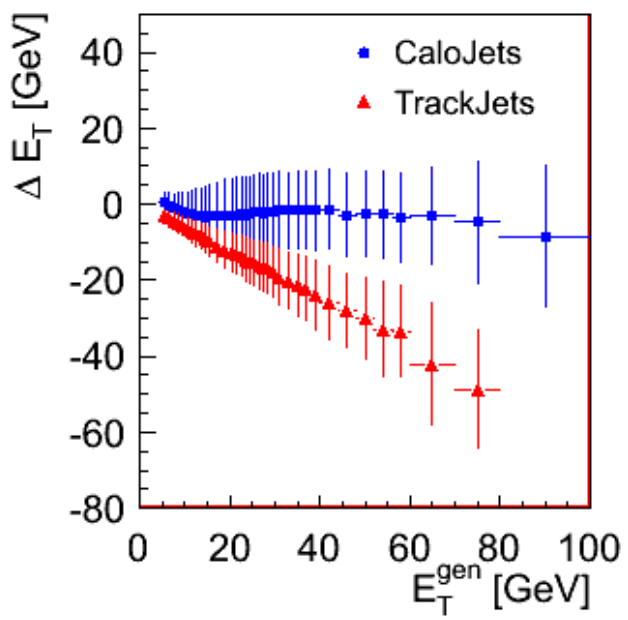

Figure 4.6: The average difference between the transverse energy of the generated and the reconstructed jet as a function of the transverse energy of the generated jet. The energy response of TrackJets (red triangles) is compared to the energy response of CaloJets (blue circles). The three levels of jet corrections are applied to the CaloJets. The errors correspond to the width of a Gaussian distribution fitted to the core of the distribution of the residuals.

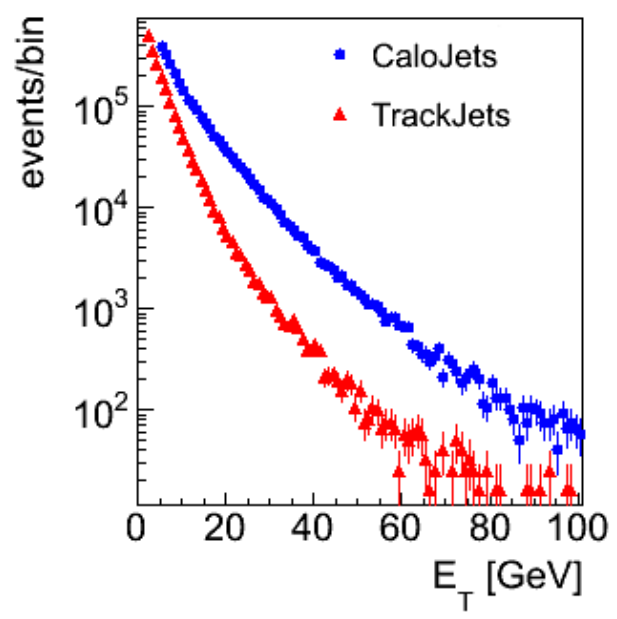

Figure 4.7: Transverse energy spectrum of $b$-jets reconstructed from TrackJets and CaloJets. The mean transverse energy is $E_{T}=5.7 \mathrm{GeV}$ for TrackJets and $E_{T}=12.2 \mathrm{GeV}$ for CaloJets.

TrackJets are reconstructed from all charged tracks with transverse momentum $p_{T}>0.3 \mathrm{GeV}$ and a minimum transverse energy of the TrackJet of $E_{T}>1 \mathrm{GeV}$ is required. In addition cuts on the number of pixel and strip detector layers with measurements are applied in order to ensure a good quality of the tracks. A summary of the selection criteria for the tracks 
used in the clustering algorithm is presented in table 4.4. The number of tracks reconstructed within a TrackJet is shown in figure 4.8 .

Table 4.4: Selection criteria for the tracks used in the TrackJet clustering algorithm.

\begin{tabular}{ll}
\hline Variable & Value \\
\hline minimum transverse momentum & $p_{T}>0.3 \mathrm{GeV}$ \\
maximum transverse momentum & $p_{T}<500 \mathrm{GeV}$ \\
longitudinal impact parameter & $z_{0}<15 \mathrm{~cm}$ \\
number of pixel layers with hits & $\geqslant 2$ \\
number of tracker layers with hits $\geqslant 5$ \\
\hline
\end{tabular}

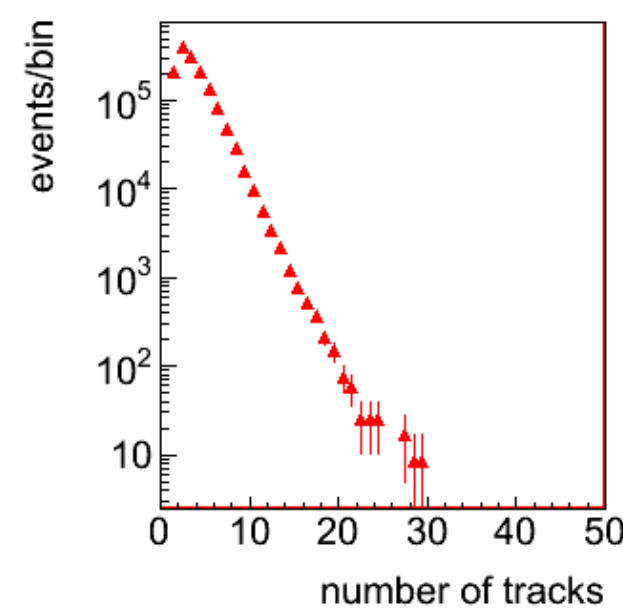

Figure 4.8: Distribution of the number of tracks reconstructed within a TrackJet.

The efficiency of associating a jet to the muon depends on the muon transverse momentum and is displayed in figure 4.9 for signal events. The efficiency is calculated for events in which a muon and a $b$-jet are present at generator level. Figure 4.9 also shows the fraction of mistagged $b$-jets as a function of the jet transverse energy. It is estimated by comparing the reconstructed jets to the generated $b$-jet. If the generated $b$-jet lies within a cone of radius $R=\sqrt{\eta^{2}+\phi^{2}}=0.5$ around the reconstructed jet, the matching is successful and the tagging is defined to be correct. The fraction of mistagged $b$-jets is obtained by dividing the number of mistagged selected jets by the total number of selected jets in signal events.

The efficiency rises from $68 \%$ to almost $100 \%$ for events containing a muon with transverse momentum $p_{T}>20 \mathrm{GeV}$. The fake rate in the lowest bin in muon transverse momentum takes values up to $10 \%$ and decreases for higher muon transverse momenta. If the measurement with data showed that a large fake rate makes the control of the background difficult, the lower cut on the transverse energy of TrackJets would have to be increased. The efficiency for CaloJets in the low muon transverse momentum bins is significantly lower compared to TrackJets, while the fake rate is higher. 
Within this study the use of TrackJets is proposed for the analysis of the very first data of CMS. At increased LHC luminosity when the focus will be on higher momentum muons the use of CaloJets as suggested in $[77,78]$ becomes interesting.
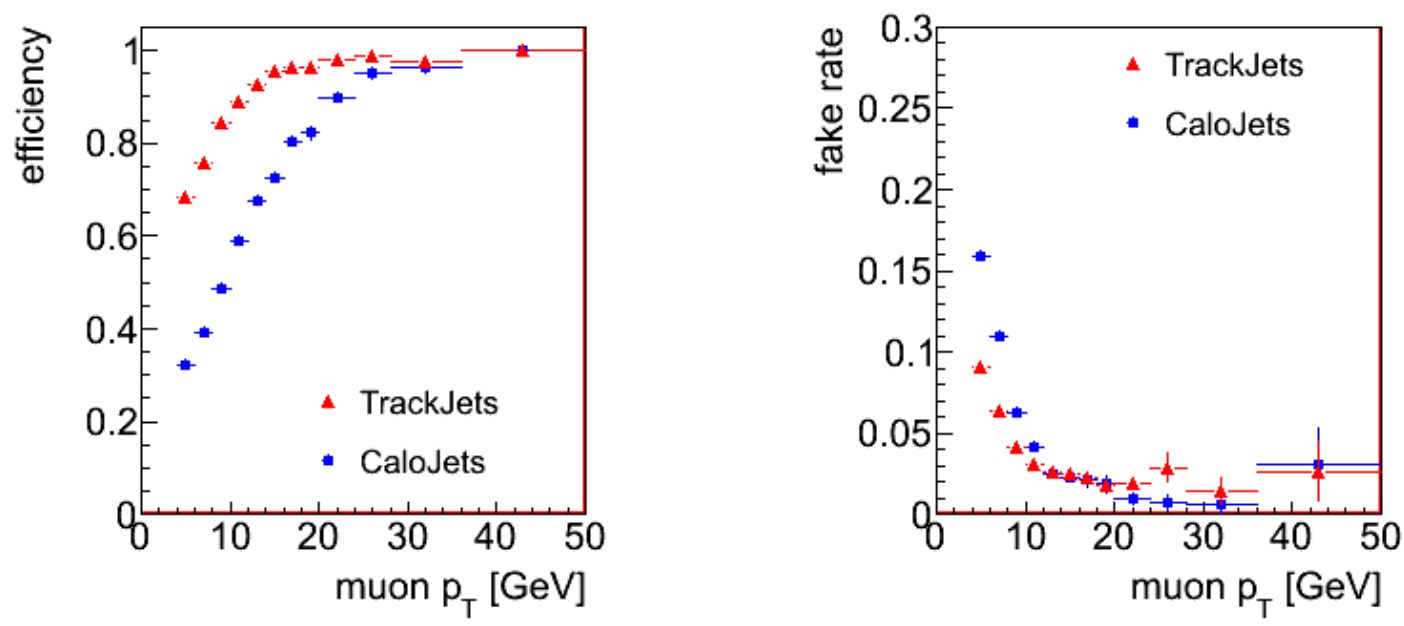

Figure 4.9: Left: Efficiency for finding a TrackJet (red triangles) or CaloJet (blue circles) in a cone of radius $R=0.5$ around the muon as a function of the muon transverse momentum. Right: Fraction of mistagged $b$-jets as a function of the muon transverse momentum.

\subsection{Event Selection}

As discussed in the previous section, the first requirement of the event selection is a reconstructed muon with transverse momentum $p_{T}>5 \mathrm{GeV}$ and pseudorapidity $-2.1<\eta<2.1$. Only global muons, i.e. muons identified in the tracking detectors as well as in the muon chambers, pass the selection. If more than one muon is present in the event, the muon with the highest transverse momentum is chosen. The transverse momentum and pseudorapidity distributions of muons passing the event selection are shown in figure 4.10 for $b$-events.

The assignment between the reconstructed muon and the TrackJet is based on the result of the TrackJet clustering algorithm. The muon is associated to a TrackJet if the muon track lies within the TrackJet with transverse energy $E_{T}>1 \mathrm{Gev}$. The distance between the muon and the TrackJet

$$
\Delta R=\sqrt{\Delta \eta^{2}+\Delta \phi^{2}}
$$

in signal and background events passing the event selection is shown in figure 4.11.

A total number of 3.7 million events are selected in a data set corresponding to $1 \mathrm{pb}^{-1}$ of integrated luminosity. Of those, $49 \%$ contain a $b$ quark while $26 \%$ are classified as $c$-events and the remaining $25 \%$ as $u d s g$-events. The variation of the $b$ fraction with the transverse momentum and the pseudorapidity of the muon is shown in figure 4.12 for simulated events.

The relative transverse momentum of the muon with respect to the axis of the associated 

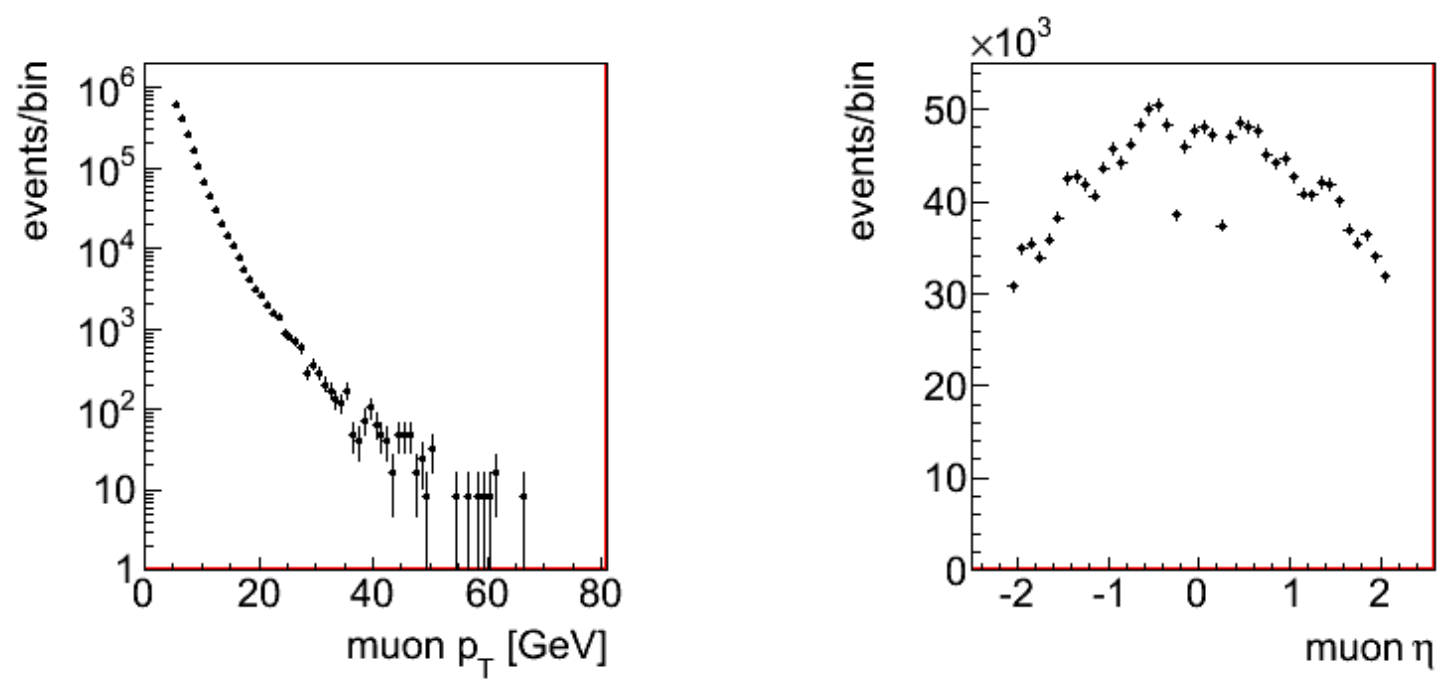

Figure 4.10: Simulated distribution of reconstructed muon transverse momentum and pseudorapidity in signal events passing the event selection. The distributions are normalized to an integrated luminosity of $1 \mathrm{pb}^{-1}$.

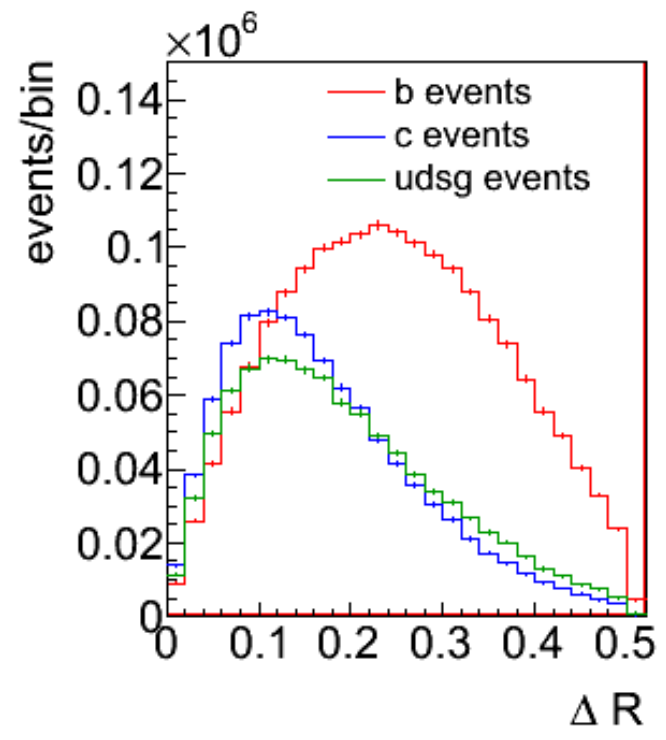

Figure 4.11: Distance between the reconstructed muon and the TrackJet $(\Delta R)$ for selected $b-, c$ - and udsg-events. The event selection requires a muon with transverse momentum $p_{T}>5 \mathrm{GeV}$ and pseudorapidity $|\eta|<2.1$ and a TrackJet with transverse energy $E_{T}>1 \mathrm{GeV}$. The distributions are normalized to an integrated luminosity of $1 \mathrm{pb}^{-1}$. 

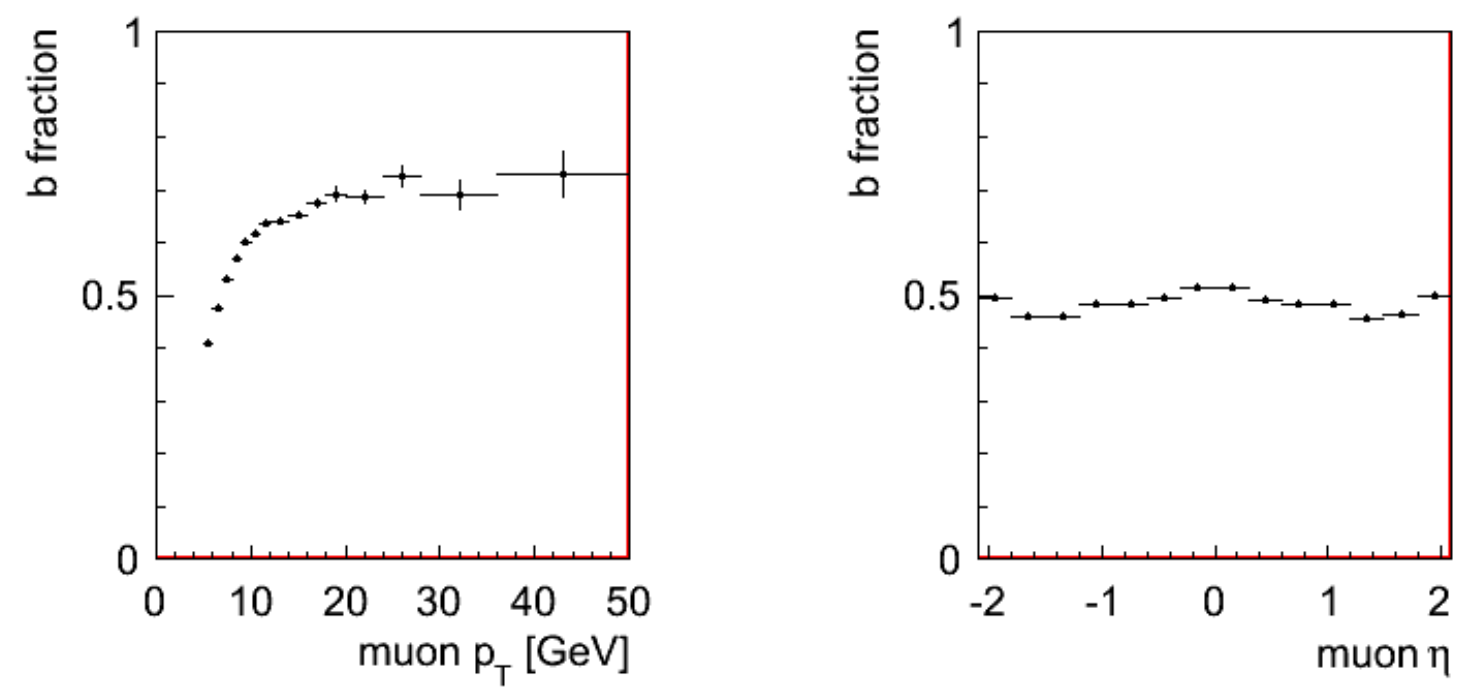

Figure 4.12: Fraction of $b$-events in the inclusive sample as a function of the transverse momentum and the pseudorapidity of the reconstructed muon.
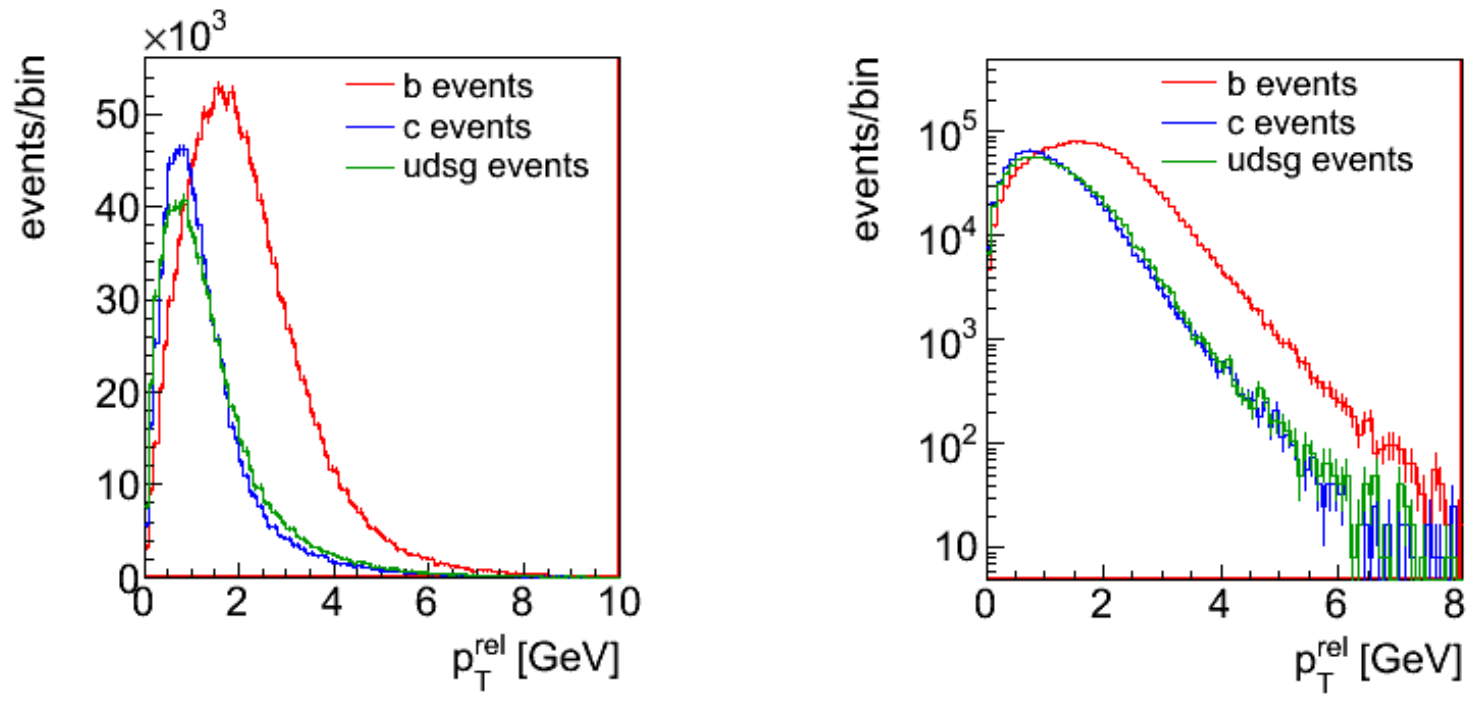

Figure 4.13: $p_{\perp}^{\text {rel }}$ distribution of the inclusive sample separated by the quark content and normalized to an integrated luminosity of $1 \mathrm{pb}^{-1}$ in selected events. The event selection requires a muon with transverse momentum $p_{T}>5 \mathrm{GeV}$ and pseudorapidity $|\eta|<2.1$ and a TrackJet with transverse energy $E_{T}>1 \mathrm{GeV}$. The left figure shows the $p_{\perp}^{\text {rel }}$ distribution on a linear scale, while in the right figure a logarithmic scale is used. 
jet is calculated according to the formula

$$
p_{\perp}^{\mathrm{rel}}=\frac{\left|\vec{p}_{\mu} \times \vec{p}_{j e t}\right|}{\left|\vec{p}_{j e t}\right|}
$$

where $\vec{p}_{\mu}$ and $\vec{p}_{j e t}$ are the momentum vector of the muon and the TrackJet, respectively.

The $p_{\perp}^{\text {rel }}$ distribution for $b$-, $c$ - and $u d s g$-events is shown in figure 4.13. The $b$-spectrum contains a contribution from cascade decays $b \rightarrow c+X \rightarrow \mu+X^{\prime}$ in addition to the direct semileptonic decays $b \rightarrow \mu+X$. The $p_{\perp}^{\text {rel }}$ spectrum of $b$-events is significantly harder than the $p_{\perp}^{\text {rel }}$ spectrum of $c$ - and $u d s g$-events, while the $c$ - and $u d s g$-spectrum turn out to be rather similar.

\subsection{Signal Extraction}

A fit to the observed $p_{\perp}^{\text {rel }}$ spectrum based on simulated templates is used to determine the fraction of signal events among all events passing the event selection. As the $p_{\perp}^{\text {rel }}$ spectrum in $c$ - and $u d s g$-events proves to be very similar, it cannot be separated in the fitting procedure. Therefore the $c$ - and $u d s g$-background component are combined and a fit with one signal component against one background component is implemented. The uncertainty of the unknown $c$ to $u d s g$ background composition is treated as a systematic uncertainty (see section 4.9).

\subsubsection{Fitting Procedure}

The estimation of the sample composition based on MC simulations of the individual sources is a common problem in experimental data analysis. The contribution of different sources is determined by fitting the binned observed data with template histograms obtained by simulation. A fitting method which takes into account the limited statistics of the MC simulation is detailed in [79] and the main points are reviewed here. The goal is to determine the scale factors $\alpha_{j}$ for each source $j$ which relate the (unknown) number of expected MC events $A_{j i}$ to the predicted number of data events $f_{i}$ in each bin $i$

$$
f_{i}=\sum_{j=1}^{m} \alpha_{j} A_{j i},
$$

where $m$ is the number of sources. Depending on the binning the number of events per bin might be quite small and the probability of observing a particular number of data events $d_{i}$ in bin $i$ is thus best described by Poisson statistics

$$
\mathcal{P}\left(d_{i} \mid f_{i}\right)=\frac{f_{i}^{d_{i}}}{d_{i} !} e^{-f_{i}}
$$

Analogous to the expected number of data events $f_{i}$, the expected number of MC events $A_{j i}$ represent the mean values of the Poisson distributed number of generated MC events $a_{j i}$ of source $j$ in bin $i$. The introduction of the unknown parameters $A_{j i}$ accounts for the fact that also the number of generated MC events fluctuates because of the limited size of the available MC samples. 
Estimates for the parameters $\alpha_{j}$ and $A_{j i}$ are determined by using the maximum likelihood technique and maximizing the negative logarithm of the likelihood function $\mathcal{L}$

$$
-\ln \mathcal{L}=\sum_{i=1}^{n} d_{i} \ln f_{i}-f_{i}+\sum_{i=1}^{n} \sum_{j=1}^{m} a_{j i} \ln A_{j i}-A_{j i} .
$$

In contrast to the results for the parameters $\alpha_{j}$ which are the scale factors we are interested in, the parameters $A_{j i}$ are only a mathematical tool and the result is of no importance for the measurement. When differentiating equation (4.3) with respect to $\alpha_{j}$ and $A_{j i}$ and setting the derivatives to zero a set of $m \times(n+1)$ non-linear and coupled equation is obtained. The equation can be simplified to derive the dependence of $A_{j i}$ on the $m$ variables $p_{j}$ in each bin separately. Thereafter the solution for $p_{j}$ can be found iteratively. The ROOT package [80] provides an implementation of the fitting procedure (TFractionFitter [81]) where the optimization problem is solved by MINUIT [82].

Special care has to be taken when weights are imposed on the MC events since the assumption that the number of MC events $a_{j i}$ obey Poisson statistics is not valid anymore. Within this analysis weights are introduced to scale the contributions of the individual $\hat{p}_{T}$ bins according to their cross section. The event weights are given by

$$
w_{k}=\frac{\sigma_{k} \cdot \mathcal{L}_{t o t}}{N_{k}},
$$

where $N_{k}$ is the number of generated events in bin $k, \sigma_{k}$ is the theoretical cross section of the corresponding simulated process and $\mathcal{L}_{\text {tot }}$ the integrated luminosity.

In the following, two procedures developed to accommodate the weights in the fitting procedure are discussed. While the first method represents an approximative treatment which is only valid if the spread of the weights among the bins is not too large, the full treatment is covered by the second method using an extension of the likelihood function.

\section{Approximate Solution}

The fitting procedure discussed above provides the possibility to introduce bin-wise weights by modifying equation (4.1) to become

$$
f_{i}=\sum_{j=1}^{m} \alpha_{j} w_{j i} A_{j i}
$$

and henceforth using equation 4.5 in the likelihood function $\mathcal{L}$.

The correct shapes of the MC template distributions for the individual sources are obtained by filling histograms with weighted events. In this case the bin contents $a_{j i}=\sum_{k} w_{k j i}$ no longer follow Poisson statistics and the bin errors are given by $\sigma_{j i}=\sqrt{\left(\sum_{k} w_{k j i}^{2}\right)}$ which is in general not equal to the square root of the bin content (the variance of the Poisson distribution). Since this is assumed in the fitting procedure, the use of those templates leads to a wrong result.

In order to properly treat the statistical uncertainties in the fit algorithm, the formalism of equivalent event numbers [83] is adopted and a set of unweighted events that have the same statistical significance as the weighted events is defined. For this purpose, each template 
histogram is transformed into a template histogram containing equivalent event numbers, $\tilde{a}_{j i}$, and a matching weight value, $\tilde{w}_{j i}$, such that the new histogram multiplied by the weights reproduces the original template content, i.e.

$$
\tilde{a}_{j i} \cdot \tilde{w}_{j i}=a_{j i} .
$$

Furthermore, the equivalent event numbers are required to be Poisson distributed and to have the same relative error as the original event numbers

$$
\frac{\tilde{\sigma}_{j i}}{\tilde{a}_{j i}}=\frac{1}{\sqrt{\tilde{a}_{j i}}}=\frac{\sigma_{j i}}{\tilde{a}_{j i}} .
$$

Equation (4.6) and (4.7) are fulfilled if

$$
\tilde{a}_{j i}=\frac{a_{j i}^{2}}{\sigma_{j i}^{2}} \quad \text { and } \quad \tilde{w}_{j i}=\frac{\sigma_{j i}^{2}}{a_{j i}} .
$$

The resulting pair of template and weight therefore describes the same distribution and uncertainties as the original template, but obeys Poisson statistics as required for the fit.

\section{Full Treatment}

In the full treatment the likelihood function is expanded to include weight factors $w_{k j i}$ representing the weights of the events of subsample $k$ from source $j$ in bin $i$. The negative logarithm is then

$$
-\ln \mathcal{L}=\sum_{i=1}^{n} d_{i} \ln f_{i}-f_{i}+\sum_{i=1}^{n} \sum_{j=1}^{m} \sum_{k=1}^{l} a_{j k i} \ln A_{j k i}-A_{j k i},
$$

where the number of expected MC events is given by

$$
f_{i}=\sum_{j=1}^{m} \sum_{k=1}^{l} \alpha_{j} w_{j k i} A_{j k i} .
$$

The solution of the optimization problem is detailed in [77]. It is shown that for any given set of $\alpha_{j}, f_{i}$ and $A_{j k i}$ can be obtained numerically and that there is a unique solution which makes all $A_{j k i}$ positive. The likelihood evaluated with these $f_{i}$ and $A_{j k i}$ then is the function to be minimized by MINUIT. A formal derivation of the equivalent event number method as an approximation to the full treatment can also be found in [77].

\subsubsection{Performance of the Fit}

The performance of the fit is studied on the basis of the MC samples generated at a center-ofmass energy of $\sqrt{s}=10 \mathrm{TeV}$ in $7 \hat{p}_{T}$ bins. An example of a fit is shown in figure 4.14. The fit is performed using two statistically independent MC subsamples. One sample is used to extract the templates of the simulated shape of the signal and background $p_{\perp}^{\mathrm{rel}}$ distributions, the other subsample is treated as "data". The plot shows the result obtained from the inclusive sample using the approximate method. The fit result well reproduces the $p_{\perp}^{\text {rel }}$ shape in "data" and 
the sample composition agrees within the error. The same statement is true when the full treatment is applied in the fitting procedure.

The dependence on the binning of the input distribution is checked by repeating the fit for different numbers of bins. The fit is found to be stable and the small deviations of the fitted scaling factors are within the statistical errors of the fit.

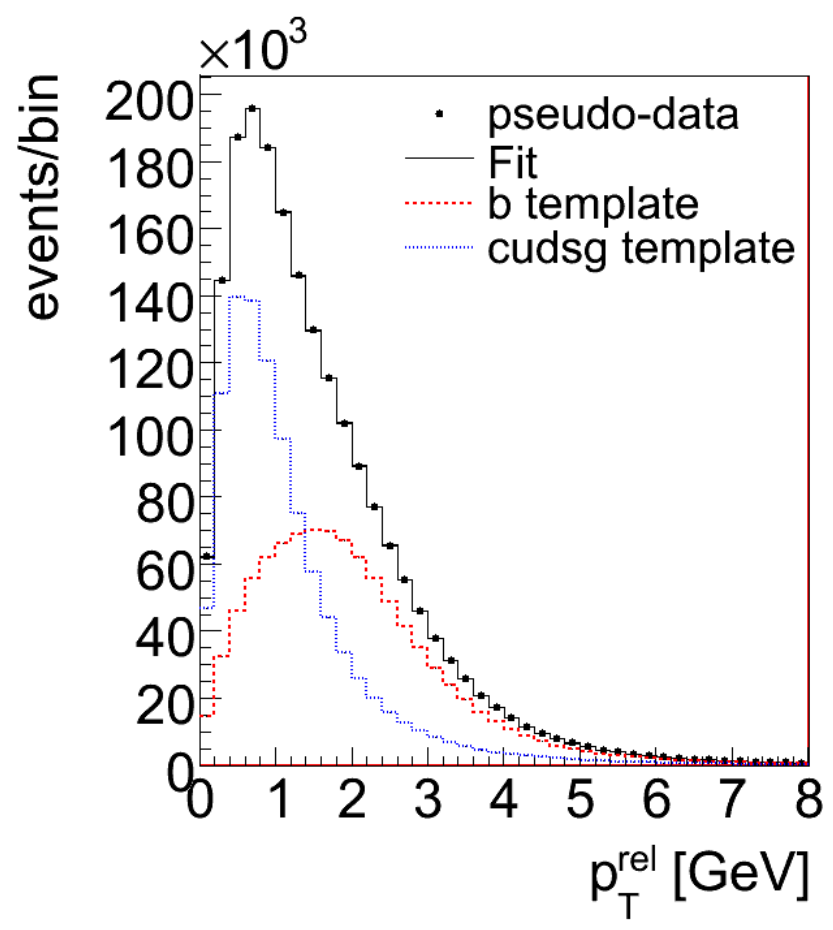

Figure 4.14: Fit result obtained by dividing the MC sample in two independent subsamples. The red dashed and the blue dotted line are the $b$ - and cudsg-template, respectively. The black full circles correspond to the data distribution, while the black line is the result of the fitting procedure using the approximate method.

In order to verify the errors calculated by the fitter and to determine a possible bias in the fit result, repeated fits of pseudo experiments are done. For repeated MC experiments it is not possible to regenerate the full detector simulation. The "truth" is therefore varied from toy experiment to toy experiment to approximately simulate the statistical uncertainty of the templates. This pseudo-truth is obtained by smearing the weighted sum of all templates according to the appropriate error. The data is then generated by Poisson fluctuations of the pseudo-truth. This method takes into account the uncertainty of the MC and the data statistics and thus presents a valid test of the fitting procedure.

In figure 4.15 the deviation of the fitted $b$-fraction from the true value is displayed for repeated fits using the approximate and the full method. In neither of the two methods a significant bias is observed and the width of the pull plots is consistent with one which implies that the errors are calculated correctly by the fitter. 

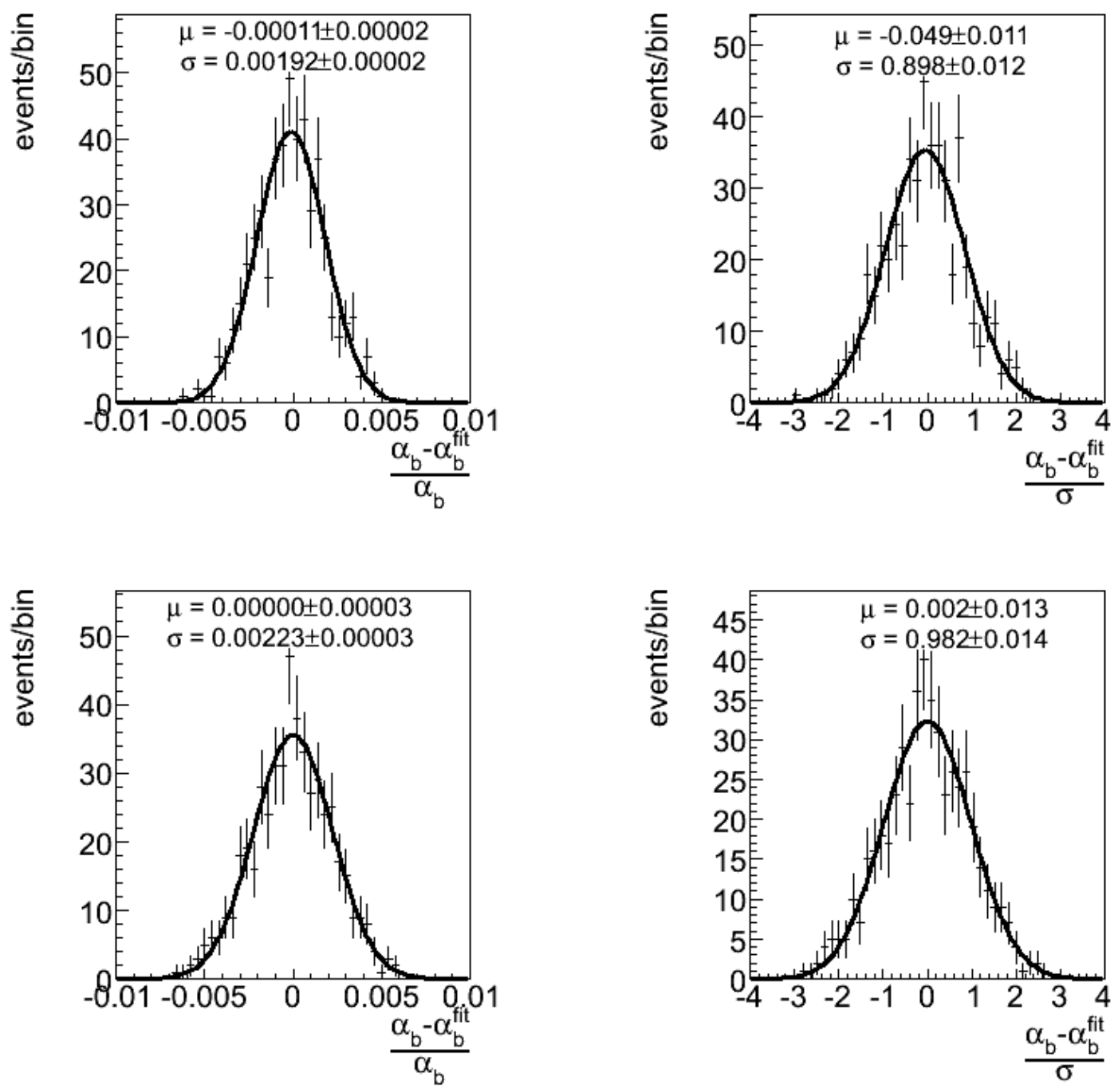

Figure 4.15: Left: Deviation of the fitted scale factor $\alpha_{b}^{\text {fit }}$ for $b$-events from the true value $\alpha_{b}$ in repeated pseudo experiments. Right: The corresponding pull distributions. The upper plots show the result for the approximate fitting method, while the lower plots represent the full treatment.

\subsection{Validation of MC Templates}

\subsubsection{Signal}

The shape of the signal $p_{\perp}^{\text {rel }}$ distribution will be validated using a data-driven method. A data sample with a high $b$-content will be used to cross-check the shape of the $p_{\perp}^{\text {rel }}$ distribution. Such a sample can be obtained for example by selecting events containing single muons with a large impact parameter. This method has been tested in MC. The signed transverse impact parameter of the muon, $d_{0}$, is calculated with respect to the jet axis. The impact parameter 
significance, $d_{0} / \sigma_{d_{0}}$, is shown in figure 4.16 for $b$-, $c$ - and $u d s g$-events. By selecting events with $d_{0} / \sigma_{d_{0}}>30$ a $b$-purity of $91 \%$ is achieved. The efficiency of the selection is $0.8 \%$ and a sample with 50000 events is obtained from data corresponding to an integrated luminosity of $1 \mathrm{pb}^{-1}$. In MC events, the shape of the $p_{\perp}^{\text {rel }}$ distribution extracted from $b$-events and from the inclusive sample with a cut on the signed transverse impact parameter significance agree within the statistical uncertainty.

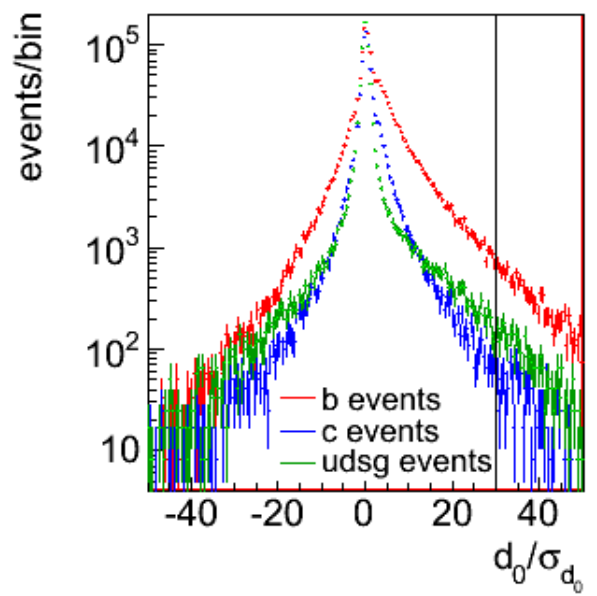

Figure 4.16: Significance of the signed transverse impact parameter of the muon with respect to the TrackJet direction in signal and background events.

\subsubsection{Background}

The light quark background is dominated by the fake muon contribution and can therefore be estimated from data. If the muon fake probability is known, the light quark background can be measured by re-weighting the hadron spectrum in minimum bias events by the fake muon probability.

While effects of punch-through hadrons mimicking a muon signature mainly become important for high momentum muons, the majority of fake muons with a transverse momentum of $p_{T}<30 \mathrm{GeV}$ is produced in in-flight decays of pions, kaons or baryons. The probability of misidentifying a pion, kaon or proton as a muon is shown in figure 4.17 as obtained from simulation. It is a function of the muon transverse momentum and has an average value of about $0.5 \%$ for kaons and $0.2 \%$ for pions. For protons the muon fake probability is very low and can be neglected in the context of this analysis.

The fake probability of kaons and pions will be measured from data adopting the method described in [84]. Therein the probability that a pion or kaon track is misidentified as a muon is evaluated using $D^{0} \rightarrow K \pi$ events. A $D^{0}$ candidate is reconstructed from any pair of charged tracks with transverse momentum $p_{T}>2 \mathrm{GeV}$. In order to reduce the combinatorial background, the $D^{0}$ meson is required to come from a semileptonic $B$ meson decay, $B^{ \pm} / B^{0} \rightarrow$ $\mu+D^{0}+X$. The presence of a muon in the event has the additional benefit of providing a clean signature in the trigger selection. $B \rightarrow \mu+D^{0}+X$ events are required to fulfill the 


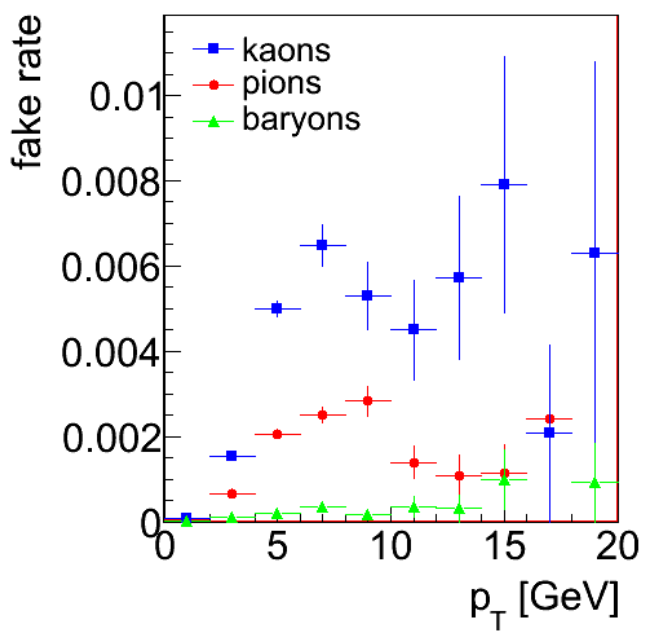

Figure 4.17: Muon fake probability for kaons, pions and baryons as obtained from the MC simulation.

following event selection:

- global muon with $p_{T}>3 \mathrm{GeV},-2.1<\eta<2.1$, accepted by HLT

- $K$ candidate: track with $p_{T}>2 \mathrm{GeV}$ and same sign as the muon,

- $\pi$ candidate: track with $p_{T}>2 \mathrm{GeV}$ and opposite sign to the muon,

- $\Delta R(\mu, K)<1$ and $\Delta R(\mu, \pi)<1$,

- secondary vertex of $K$ - and $\pi$-track with quality $\chi^{2}<10$

- invariant mass $m_{D^{0}}$ of the $K$ - and $\pi$-candidate in $1.6 \mathrm{GeV}<m_{D^{0}}<2.1 \mathrm{GeV}$.

The number of signal events is then determined by performing a fit to the invariant mass spectrum of the candidate events. The $D^{0}$ mass peak is fitted with a Gaussian function with a mean of $1.865 \mathrm{GeV}$ and a width of $15 \mathrm{MeV}$. The shape of the background is assumed to be exponential and the parameters are extracted by a fit in the side-bands at $m_{D^{0}}<1.8 \mathrm{GeV}$ and $m_{D^{0}}>1.92 \mathrm{GeV}$. The fake muon probability for pions and kaons is derived using the invariant mass distribution of $D^{0} \rightarrow K \pi$ events in which one of the decay products is reconstructed as the track belonging to a global muon. In these events the invariant mass spectrum is fitted using the same procedure as described before to determine the number of events in the peak. The fake rate is then given by the number of signal events where one leg is misidentified as a muon divided by the total number of signal events. It can be determined separately for pions and kaons, as the opposite charge allows to distinguish the decay products. The method is tested with the help of a MC sample corresponding to an integrated luminosity of $\mathcal{L}=0.1 \mathrm{pb}^{-1}$. In this sample $1300 D^{0}$ candidates are reconstructed in the signal peak and 5 events with a misidentified kaon are found. These numbers reproduce the kaon fake probability in the MC simulation within the statistical error of $50 \%$. In the case of pions, 
the available MC statistics is too low to determine the fake probability and it is concluded that a measurement is possible with a data set corresponding to an integrated luminosity of $\mathcal{L}>0.2 \mathrm{pb}^{-1}$. The fitted invariant mass distribution for $D^{0} \rightarrow K \pi$ events is shown in figure 4.18 (left), while the subset of events where the kaon is misidentified as a muon is presented in figure 4.18 (right).
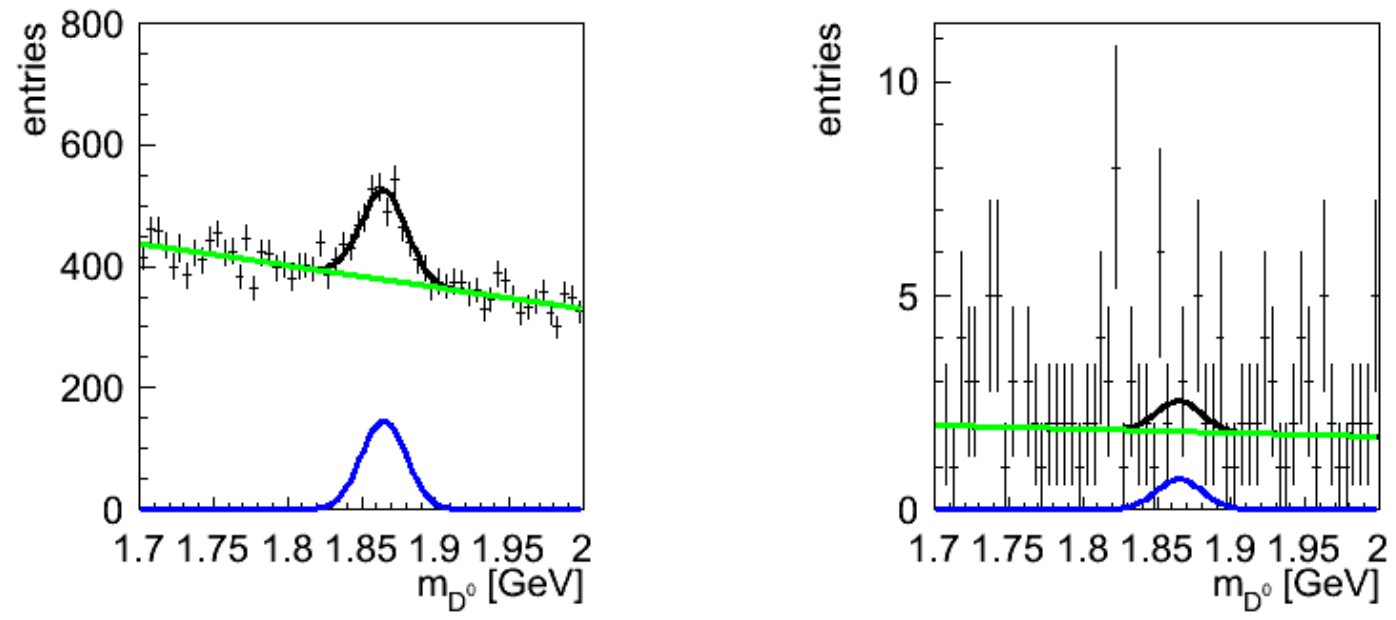

Figure 4.18: Left: fitted invariant mass distribution of $D^{0}$ candidates. Right: fitted invariant mass distribution of $D^{0}$ candidates where the kaon leg is misidentified as a muon.

In order to calculate the muon probability for tracks in an inclusive data sample, the composition of the minimum bias track spectrum has to be known. This information is taken from the MC simulation where the hadronic spectrum consists of $62 \%$ pions, $15 \%$ kaons and $19 \%$ baryons. The fake probability depends on the track transverse momentum. A data set corresponding to a luminosity of $\mathcal{L}>10 \mathrm{pb}^{-1}$ would be needed to measure the shape of the transverse momentum dependence from data. As this amount of data will not be available within the time scale of this analysis, we propose to take the shape of the fake probability from simulation and measure the absolute normalization from data.

The scaling of the hadronic track spectrum with the muon fake probability has been performed using MC events. For this study a sample of minimum bias events generated by PYTHIA with a number of events corresponding to an integrated luminosity of $0.2 \mathrm{nb}^{-1}$ was used. The transverse momentum and pseudorapidity distributions obtained by re-weighting the hadronic track spectrum is compared to the corresponding distributions of fake muons in figure 4.19. Furthermore, the $p_{\perp}^{\mathrm{rel}}$ spectrum calculated from re-weighted tracks and the closest TrackJet is compared to the $p_{\perp}^{\text {rel }}$ spectrum of fake muons. A good agreement is found for the transverse momentum and the $p_{\perp}^{\text {rel }}$ spectrum, while the pseudorapidity distributions of fake muons and re-weighted tracks differ significantly. The discrepancy is due to the fact that the fake probability used for re-weighting the hadronic track spectrum is a function of transverse momentum only and the pseudorapidity dependence of the fake probability is not taken into account. This however does not have an effect on the shape of the re-weighted $p_{\perp}^{\mathrm{rel}}$ spectrum in the simulation. When using the re-weighted $p_{\perp}^{\mathrm{rel}}$ spectrum as light quark 
template in the fitting procedure, the resulting $b$ fraction agrees with the fraction obtained from $\mathrm{MC}$ within the statistical error. It is concluded that the method of scaling the minimum bias track spectrum is well suited for measuring the light quark background from data.
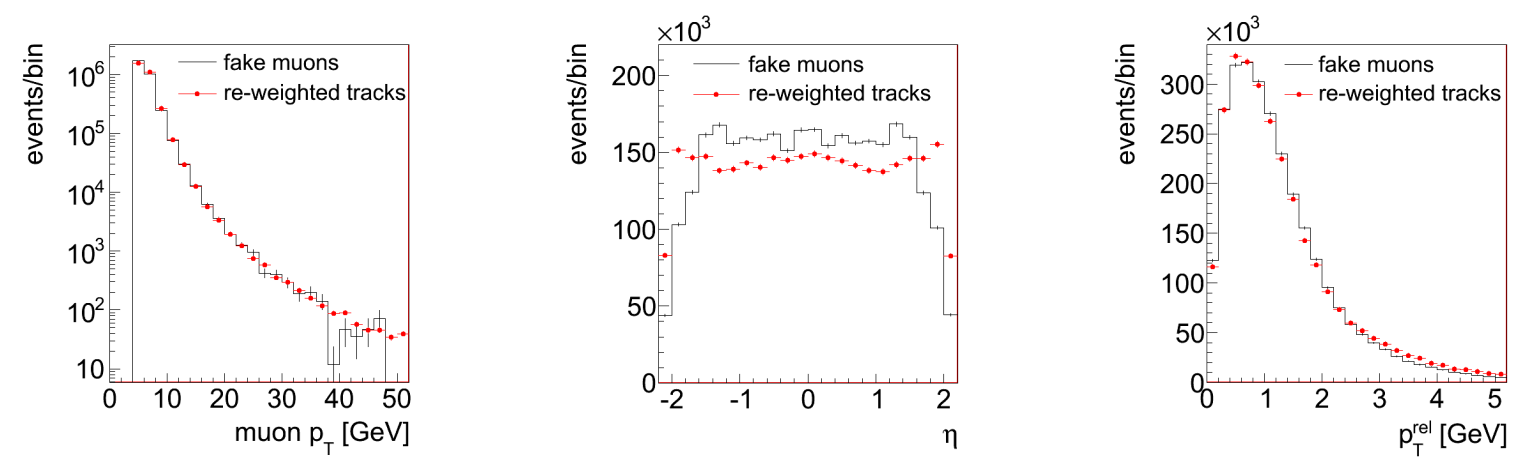

Figure 4.19: Simulated hadronic track spectrum re-weighted by the fake probability obtained from MC and compared to the simulated fake muon spectrum: transverse momentum (left), pseudorapidity (center) and $p_{\perp}^{\text {rel }}$ distribution (right).

\subsection{Cross Section Measurement}

\subsubsection{Inclusive Cross Section}

The inclusive $b$-quark production cross-section $\sigma$ is calculated according to the formula

$$
\sigma\left(p p \rightarrow b+X \rightarrow \mu+X^{\prime}, p_{T}^{\mu}>5 \mathrm{GeV},\left|\eta^{\mu}\right|<2.1\right)=\frac{\alpha_{b} \cdot N_{b, r e c}^{M C}}{\mathcal{L} \cdot \varepsilon},
$$

where $\alpha_{b}$ is the scale factor for $b$-events determined by the fit, $N_{b, r e c}^{M C}$ is the number of MC events passing the event selection, $\mathcal{L}$ is the integrated luminosity and $\varepsilon$ is the efficiency of the trigger and offline selection.

The efficiency of the trigger and offline selection is obtained from the MC simulation as

$$
\varepsilon=\frac{N_{b, r e c}^{M C}}{N_{b, g e n}^{M C}},
$$

where $N_{b, g e n}^{M C}$ denotes the number of generated $b$-events in the visible kinematic range. The efficiency is found to be $\varepsilon=65 \%$ where the main factors are the trigger efficiency $(79 \%)$ and the efficiency of associating a TrackJet to the reconstructed muon (81\%).

\subsubsection{Differential Cross Section}

The differential $b$-quark production cross-section is measured as a function of muon transverse momentum and pseudorapidity. The flavor composition is determined by performing a fit in each analysis bin. The binning is chosen such that the number of events in every bin is sufficient for a stable fit and at the same time a maximum purity is achieved. The purity $\mathcal{P}$ 
is defined as the fraction of selected reconstructed events in each bin that have the respective generated quantity in the same bin and is calculated based on the MC simulation:

$$
\mathcal{P}=\frac{N_{\text {rec } \rightarrow \text { gen }}^{i, M C}}{N_{\text {rec }}^{i, M C}}
$$

Here $N_{\text {rec }}^{i, M C}$ is the number of reconstructed MC events with muon transverse momentum (or pseudorapidity) in bin $i$. The reconstrutced muons are matched to generated muons (based on $\Delta R$ ) in order to determine $N_{r e c \rightarrow g e n}^{i, M C}$, i.e. the number of events with both reconstructed and generated muon transverse momentum (pseudorapidity) in bin $i$. The purity takes into account bin-to-bin migration caused by resolution effects and contributions from fake muons in $b$-events.

The muon transverse momentum range from 5 to $50 \mathrm{GeV}$ is divided into 10 non-equidistant bins with bin sizes between 1 and $20 \mathrm{GeV}$. The muon pseudorapidity range, from -2.1 to 2.1 , is covered by 14 equidistant bins with a bin size of 0.3 . A total of $10^{3}-10^{5}$ reconstructed signal events are expected in each bin in a dataset corresponding to $1 \mathrm{pb}^{-1}$ of integrated luminosity. The purity is shown in figure 4.20 as a function of muon transverse momentum and pseudorapidity. It is higher than $90 \%$ in all bins in muon transverse momentum and above $99 \%$ in all bins of muon pseudorapidity.
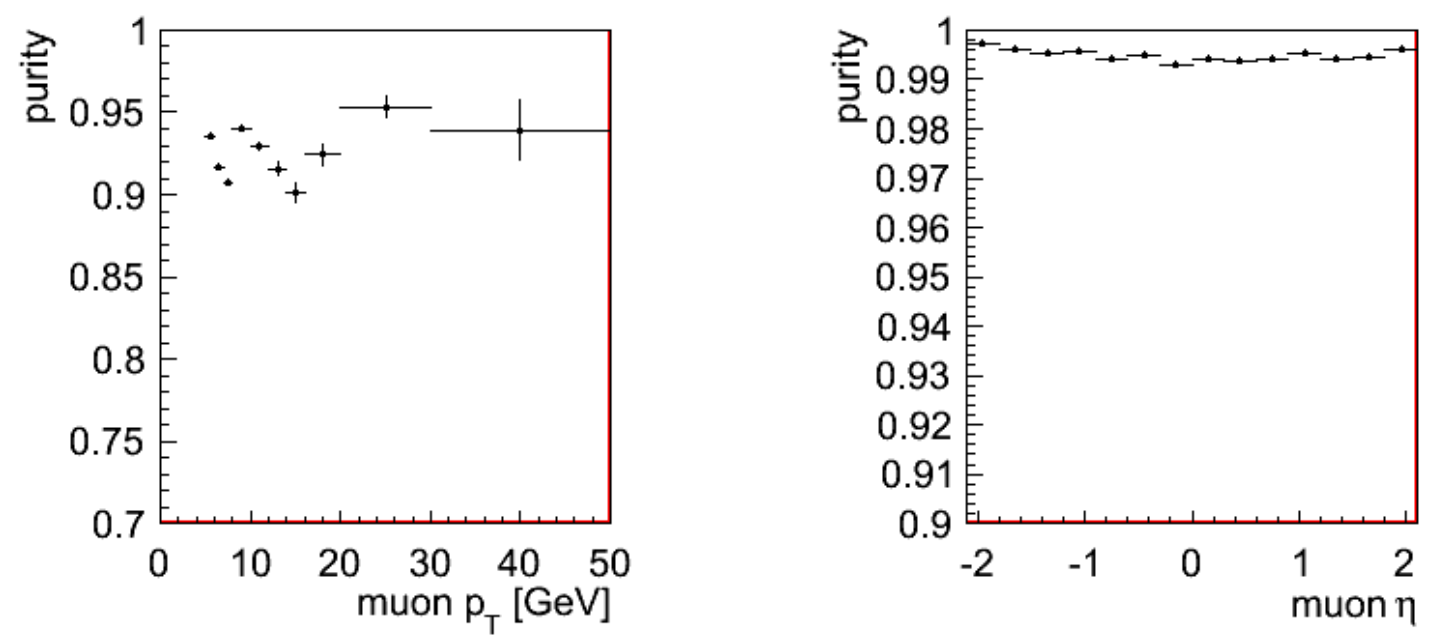

Figure 4.20: Purity for $b$-events in bins of muon transverse momentum (left) and pseudorapidity (right). The purity takes into account the contribution from fake muons and resolution effects.

The templates for the fraction fit are determined individually for each bin. While the $p_{\perp}^{\mathrm{rel}}$ distributions are similar in all bins of muon pseudorapidity, a shift to higher $p_{\perp}^{\mathrm{rel}}$ values is observed in the bins corresponding to higher muon transverse momenta. The templates and the fit results are shown in appendix A for all bins. 
The differential cross sections $\frac{d \sigma}{d p_{T}}$ and $\frac{d \sigma}{d \eta}$ are given by

$$
\begin{gathered}
\left.\frac{d \sigma}{d p_{T}}\right|_{\text {bin } i}\left(p p \rightarrow b+X \rightarrow \mu+X^{\prime},\left|\eta^{\mu}\right|<2.1\right)=\frac{\alpha_{b}^{i} \cdot N_{b, r e c}^{i, M C}}{\mathcal{L} \cdot \varepsilon^{i} \cdot \Delta p_{T}}, \\
\left.\frac{d \sigma}{d \eta}\right|_{\text {bin } i}\left(p p \rightarrow b+X \rightarrow \mu+X^{\prime}, p_{T}^{\mu}>5 \mathrm{GeV}\right)=\frac{\alpha_{b}^{i} \cdot N_{b, r e c}^{i, M C}}{\mathcal{L} \cdot \varepsilon^{i} \cdot \Delta \eta},
\end{gathered}
$$

where $\alpha_{b}^{i}, N_{b, \text { rec }}^{i, M C}$ and $\varepsilon^{i}$ refer to the quantities defined in equation (4.11), though evaluated in bin $i . \Delta p_{T}$ and $\Delta \eta$ are the widths of the transverse momentum and pseudorapidity bins, respectively. The trigger and offline event selection efficiency in the individual bins is displayed in figure 4.21. The efficiency rises as a function of muon transverse momentum from about $50 \%$ at $p_{T}=5 \mathrm{GeV}$ to $90 \%$ for $p_{T}>20 \mathrm{GeV}$. The shape of the efficiency as a function of pseudorapidity is explained by the shape of the L1 efficiency of HLT_Mu5.
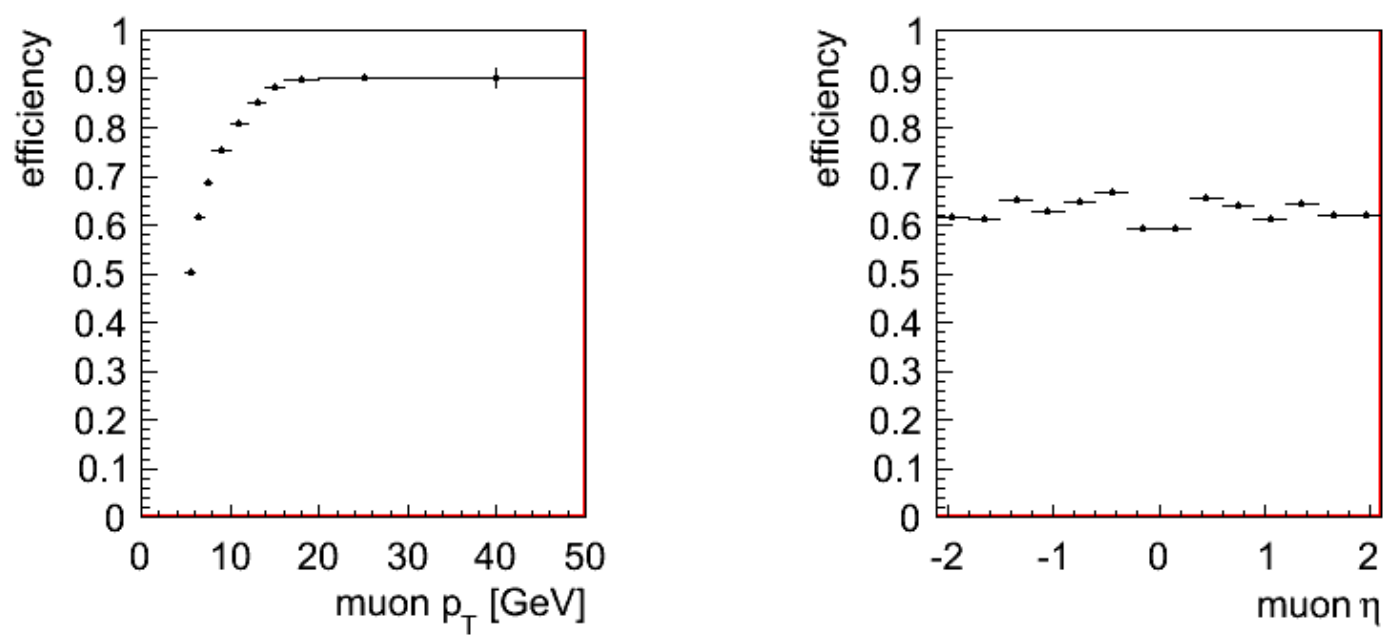

Figure 4.21: Combined efficiency of the online and offline event selection as a function of muon transverse momentum (left) and pseudorapidity (right). The efficiency is obtained from simulation.

\subsection{Systematic Uncertainties}

Due to the high $b$-quark production cross-section, the uncertainty of the measurement will quickly become systematics dominated. In this chapter we cover the main systematic uncertainties that are expected to influence the measured cross section. Some of the systematic uncertainties are expected to be provided by other groups, others will be measured from data directly. The effects of the uncertainty of the detector description as well as the uncertainty on the dynamics of the process (production, fragmentation, decay) are taken into account. In addition, the systematic uncertainty arising from the fitting procedure and the limited number of MC simulations is discussed. 


\subsubsection{Trigger}

In this study we assume an uncertainty of $5 \%$ for the L1 and HLT efficiency. An additional systematic uncertainty of $3 \%$ is assigned for the muon reconstruction efficiency. The muon trigger efficiency and its uncertainty will be determined from data directly by dedicated study groups using the "tag \& probe" method [85]. Therein the known mass resonance of the $J / \psi$ is utilized to select muon candidates and to probe the trigger efficiency. Assuming no efficiency correlation between the two decay products of the resonance it identifies one of the muons (tag) using tight identification criteria and measures the efficiency of the other muon (probe).

\subsubsection{Tracking Efficiency and Misalignment}

The TrackJet reconstruction algorithm uses all tracks with $p_{T}>0.3 \mathrm{GeV}$ as input. A potential misalignment of the tracking detectors in the early CMS data taking might degrade the performance of the track reconstruction. The actual tracking efficiency might be lower than the tracking efficiency assumed in the detector simulation. The impact of the tracking inefficiency on the TrackJet reconstruction efficiency as well as on the angular resolution is estimated in this study.

The tracking inefficiency is emulated on generator level by discarding a certain fraction of generated charged particles within the tracker volume. The reconstruction efficiency for muons is significantly higher than for pions. Generated muons are thus treated separately and are not rejected in the generated event. The remaining charged particles are used as input to the TrackJet reconstruction algorithm. The reconstruction efficiency and the angular resolution are investigated for different fraction of discarded charged particles corresponding to tracking inefficiencies from $0-20 \%$.

The efficiency for finding a TrackJet close to the reconstructed muon as a function of the tracking inefficiency is shown in figure 4.22 (left) for signal events. Since the tracking efficiency has been measured with a precision of $4 \%$ [86], a systematic uncertainty of $2 \%$ on the number of events which pass the event selection is attributed to the cross-section measurement. The effect of the tracking inefficiency on the jet angular resolution is reflected in the $p_{\perp}^{\text {rel }}$ distribution (figure 4.22 , right). In order to get an estimate of the systematic uncertainty of the cross section, the fitting procedure is repeated using the different $p_{\perp}^{\text {rel }}$ distributions as MC templates for the signal events. The fitted $b$-fraction deviates by $1 \%$ when varying the tracking efficiency within its uncertainty.

\subsubsection{Background Composition}

The $c$ - and $u d s g$-content of the sample cannot be determined separately by the fit. If the $c$-fraction of the non- $b$ background in data is different from the value used in composing the templates, the fitted $b$-fraction may vary slightly. The MC simulation predicts a $c$-fraction of $50-70 \%$ in the non- $b$ background depending on the muon transverse momentum. The $u d s g$ background is obtained from the inclusive sample and the contribution from fake muons is taken into account. Figure 4.23 shows the results of the fitted $b$-fraction when varying the $c$-fraction of the non- $b$ background in the "data" distribution from 0 to 1 while keeping the $c$-fraction in the background template constant at the value predicted by the MC simulation. The plot is obtained using the statistics of the full samples in the fit. The red lines are at the $\mathrm{MC}$ value of the $b$-fraction in the inclusive sample and the $c$-fraction in the non- $b$ background. 

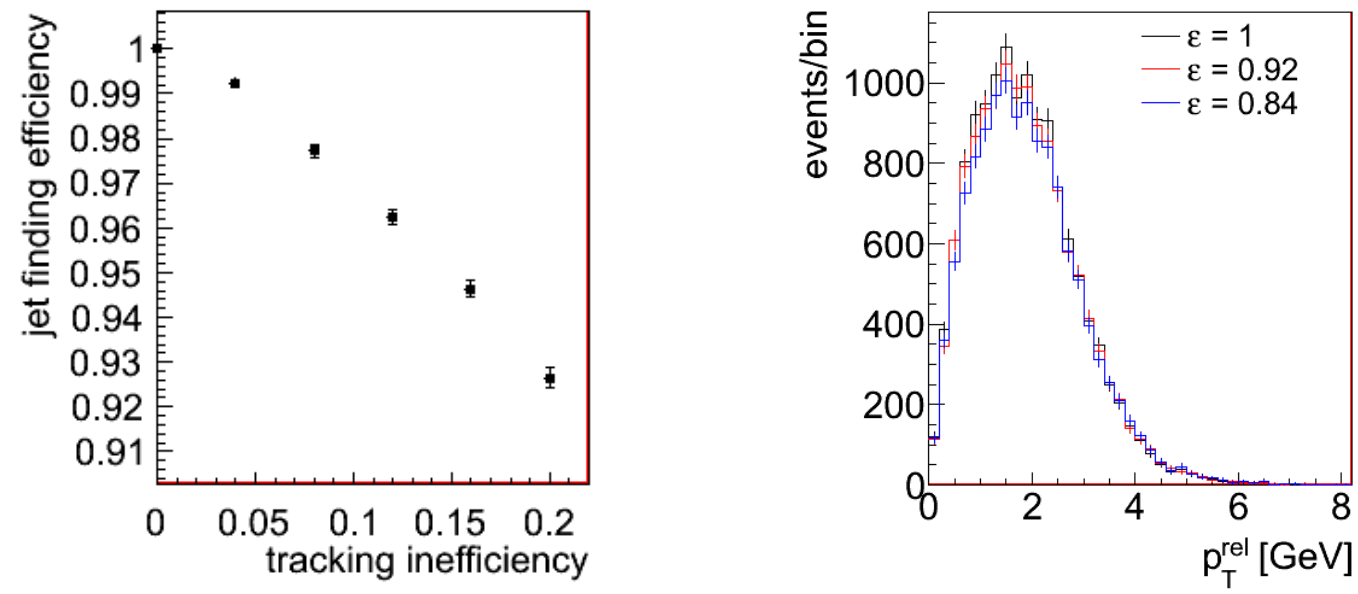

Figure 4.22: Jet finding efficiency as a function of the tracking inefficiency in signal events (left) and shape of the $p_{\perp}^{\text {rel }}$ distribution in signal events for different values of the tracking efficiency (right).

The uncertainty on the composition can in principle be constrained using data. Non-cbackground, in contrast to $c$-background, will be dominated by fake muons and can therefore be estimated. If the muon fake probability is known, the non-c-background can be measured from data by scaling the hadron spectrum in minimum bias events by the muon fake probability. As discussed in section 4.7, the non-c-background can be obtained from a dataset corresponding to an integrated luminosity of $0.2 \mathrm{pb}^{-1}$ with an accuracy of $50 \%$. The uncertainty of the $c$-fraction in the non- $b$ background is indicated by the yellow box in figure 4.23. In this region the $b$-fraction determined by the fitter changes by $3-6 \%$ depending on the muon transverse momentum and pseudorapidity.

\subsubsection{Fragmentation and Decay}

About $10 \%$ of the $b$-hadron decays contain a muon from the decay of a $c$-hadron. These sequential muons carry a smaller fraction of the original $b$-quark momentum and have a softer momentum and $p_{\perp}^{\text {rel }}$ spectrum. The fraction of sequential muons in the $b$-sample affects the overall efficiency and the shape of the $p_{\perp}^{\text {rel }}$ spectrum. Varying this fraction within its uncertainty [49] changes the measured cross section by $1 \%$.

Furthermore, the effects of alternative modeling of $b$-quark fragmentation or $b$-hadron decays are investigated. The hardness of the fragmentation and the nature of hadronic decays of the $b$-hadron are expected to have an influence on the transverse momentum spectrum of the muon. Moreover, the description of the fragmentation and the decay might affect the performance of the TrackJet clustering algorithm and consequently the reconstruction efficiency and the angular resolution.

A sample generated with EvtGen is used to investigate the effect of the $b$-hadron decay properties. The $b$-quark fragmentation in the PYTHIA sample is modeled by the Peterson fragmentation function and the parameter $\epsilon_{b}$ defines the hardness of the fragmentation. The 


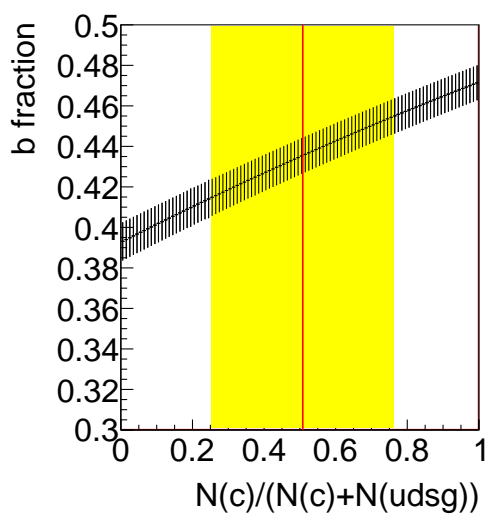

Figure 4.23: Result of the fitted $b$-fraction when varying the $c$-fraction in the background in the "data" distribution while keeping the $c$-fraction in the background template constant at the value predicted by the MC simulation. The red line is at the MC value of the $c$-fraction in the background. The yellow box indicates the uncertainty of the $c$-fraction in the background. A systematic uncertainty of the fitted $b$-fraction of $5 \%$ is determined in the inclusive sample.

uncertainty of the fragmentation is studied by varying the parameter $\epsilon_{b}$ between 0.003 and 0.010 .

The muon trigger efficiency changes by less than $1 \%$ when changing the description of the fragmentation and the decay. This effect can therefore be neglected.

The stability of the TrackJet clustering algorithm is tested by running it on generator level on events produced with the different generator settings. It is found that the efficiency of finding a TrackJet close to the reconstructed muon does not depend on the fragmentation and changes by $1 \%$ when simulating the decay with EvtGen.

The relative transverse momentum of the muon with respect to the TrackJet does depend on the modeling of the fragmentation and the decay. In order to quantify the effect, the shapes of the $p_{\perp}^{\text {rel }}$ distribution obtained from the different samples are again used in the fitting procedure. A change by $1-4 \%$ is observed for the value of the fitted $b$ fraction.

The number of $b \rightarrow \mu+X$ events inside the acceptance of this analysis depends, in addition to the $b$-quark production cross-section itself, on the hardness of the fragmentation and the decay branching fractions of $b$-hadrons. These numbers will be relevant for a comparison with $b$-quark production calculations but are not systematic uncertainties for a measurement of $\sigma\left(p p \rightarrow b+X \rightarrow \mu+X^{\prime}\right)$.

\subsubsection{Production Mechanism}

The main production mechanisms that contribute to the $b \bar{b}$ production at LO are flavor creation (hard QCD scattering), flavor excitation (semi-hard process) and gluon splitting (soft process). Since the angle between the two $b$ quarks is smaller in gluon splitting events than in flavor creation or flavor excitation events, the probability of including the two $b$ quarks in the same jet is higher. Thus, the average transverse energy of the $b$-jet is higher in gluon splitting events. This has an effect on the event selection efficiency as well as on the shape 
of the $p_{\perp}^{\text {rel }}$ distribution. The transverse momentum of the muon, the transverse energy of the jet and the $p_{\perp}^{\text {rel }}$ distribution are shown in figure 4.24 for the three production mechanisms.

In the events passing the event selection a fraction of $19 \%$ are produced by flavor creation, $56 \%$ by flavor excitation and the remaining $25 \%$ by gluon splitting. The total event selection efficiency for the three production processes are $62 \%$ for flavor creation, $64 \%$ for flavor excitation and $67 \%$ for gluon splitting events.

The contribution of the three mechanisms to $b \bar{b}$ production is predicted by the MC simulation. To estimate the uncertainty of the relative contributions of the three mechanisms we compare the PYTHIA prediction with the HERWIG prediction and take the difference as the uncertainty. The values obtained in bins of transverse momentum are listed in table 4.5.

The signal events were then re-weighted in order to adjust the fraction of the three production mechanisms to the value determined by HERWIG. The overall efficiency of the event selection was found to change by less than $1 \%$ in all bins. The fitted $b$-fraction changes by $2-5 \%$ depending on the bin in muon transverse momentum.
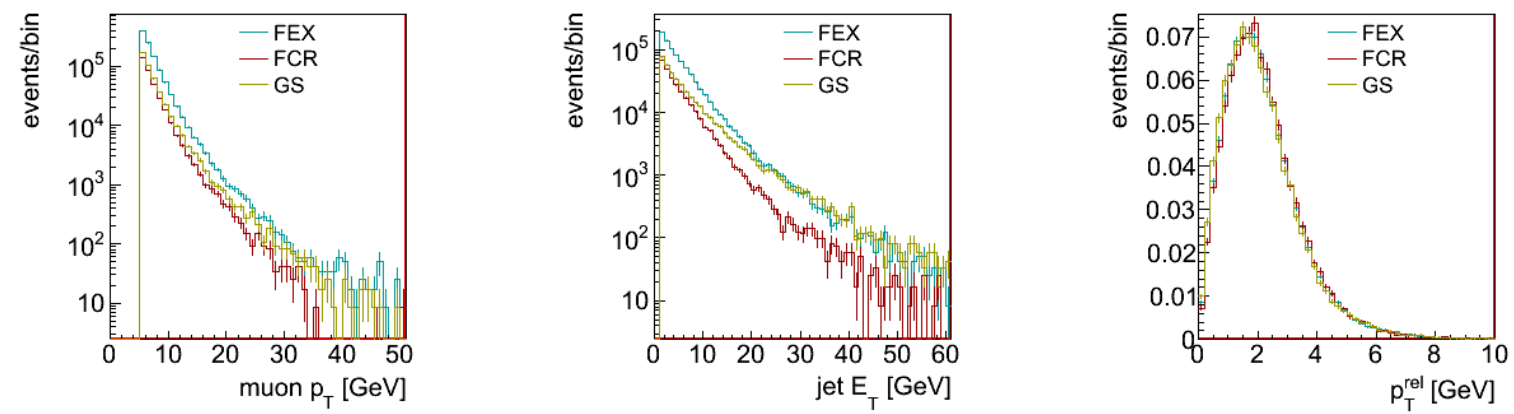

Figure 4.24: Muon transverse momentum (left), TrackJet transverse energy (center) and $p_{\perp}^{\mathrm{rel}}$ distribution (right) in flavor excitation (FEX), flavor creation (FCR) and gluon splitting (GS) events.

\subsubsection{Description of the Underlying Event}

Tracks from the underlying event can change the properties of TrackJets associated to the muon. Especially in events with low multiplicity TrackJets, the distribution of additional tracks may influence the TrackJet reconstruction efficiency and angular resolution. The systematic uncertainty due to the description of the underlying event has been studied on the basis of simulated events generated with different MC tunes. They were fit with the standard templates and the observed variation was negligible. The selection efficiency changes are of the order of $10 \%$ [87].

\subsubsection{Monte Carlo Statistics}

The fact that the number of simulated events is limited leads to a systematic uncertainty on the measured $b$-quark production cross-section. The extent of this effect can be estimated by considering the results of the validation of the fitting procedure (figure 4.15). An overview of 
Table 4.5: PYTHIA and HERWIG prediction for the relative contributions of the three production mechanisms (flavor creation, flavor excitation, gluon splitting) to the $b \bar{b}$ production.

\begin{tabular}{|l|c|c|c|c|c|c|}
\hline$p_{T}^{\mu}$ & \multicolumn{2}{|c|}{ flavor creation } & \multicolumn{2}{c|}{ flavor excitation } & \multicolumn{2}{c|}{ gluon splitting } \\
\hline & PYTHIA & HERWIG & PYTHIA & HERWIG & PYTHIA & HERWIG \\
\hline $5-6 \mathrm{GeV}$ & $19 \%$ & $23 \%$ & $56 \%$ & $52 \%$ & $25 \%$ & $25 \%$ \\
$6-7 \mathrm{GeV}$ & $19 \%$ & $22 \%$ & $56 \%$ & $55 \%$ & $25 \%$ & $23 \%$ \\
$7-8 \mathrm{GeV}$ & $19 \%$ & $20 \%$ & $56 \%$ & $55 \%$ & $25 \%$ & $25 \%$ \\
$8-10 \mathrm{GeV}$ & $19 \%$ & $23 \%$ & $56 \%$ & $55 \%$ & $25 \%$ & $22 \%$ \\
$10-12 \mathrm{GeV}$ & $19 \%$ & $21 \%$ & $56 \%$ & $49 \%$ & $25 \%$ & $30 \%$ \\
$12-14 \mathrm{GeV}$ & $18 \%$ & $26 \%$ & $55 \%$ & $48 \%$ & $27 \%$ & $26 \%$ \\
$14-16 \mathrm{GeV}$ & $18 \%$ & $25 \%$ & $54 \%$ & $51 \%$ & $28 \%$ & $24 \%$ \\
$16-20 \mathrm{GeV}$ & $19 \%$ & $17 \%$ & $53 \%$ & $56 \%$ & $28 \%$ & $27 \%$ \\
$20-30 \mathrm{GeV}$ & $18 \%$ & $20 \%$ & $52 \%$ & $39 \%$ & $30 \%$ & $41 \%$ \\
$30-50 \mathrm{GeV}$ & $10 \%$ & $17 \%$ & $55 \%$ & $34 \%$ & $35 \%$ & $49 \%$ \\
\hline
\end{tabular}

the relative error of the fitted $b$-fraction is presented in appendix B. The error of the fitted $b$-fraction takes into account the limited MC statistics as well as the limited data statistics.

\subsubsection{Luminosity}

The determination of the integrated luminosity and its uncertainty is crucial for this measurement. Different methods are proposed to measure the integrated luminosity at CMS. The quality of the luminosity measurement is a dominate source of systematic uncertainty for the cross section measurement. During the early CMS data taking, the integrated luminosity has been determined with a precision of $11 \%$ [88].

\subsubsection{Summary}

A summary of the main systematic uncertainties of the measurement of the $b$-quark production cross-section is shown in table 4.6. The main contribution is due to the uncertainty of the integrated luminosity. The systematic uncertainties depend on the muon transverse momentum and pseudorapidity bin.

\subsection{Results}

Within the analysis presented here the events of interest are selected by requiring a global muon with transverse momentum $p_{T}>5 \mathrm{GeV}$ and pseudorapidity $|\eta|<2.1$ and a TrackJet with transverse energy $E_{T}>1 \mathrm{GeV}$ in the reconstructed event.

A sample of 1.8 million selected $b$-events and about the same number of background events is expected when analyzing proton-proton collision data at a center-of-mass energy of $\sqrt{s}=7 \mathrm{TeV}$ corresponding to an integrated luminosity of $1 \mathrm{pb}^{-1}$. A maximum likelihood fit to the observed $p_{\perp}^{\mathrm{rel}}$ spectrum based on MC templates of the $p_{\perp}^{\mathrm{rel}}$ spectrum in signal and background events is performed in order to determine the number of $b$-events among the selected events. 
Table 4.6: Summary of systematic uncertainties

\begin{tabular}{lr}
\hline source & uncertainty \\
\hline Trigger & $5 \%$ \\
Muon reconstruction efficiency & $3 \%$ \\
Tracking efficiency & $2 \%$ \\
Background composition & $3-6 \%$ \\
Fragmentation & $4 \%$ \\
Decay & $3 \%$ \\
Production mechanism & $2-5 \%$ \\
Underlying event & $10 \%$ \\
MC statistics & $1-4 \%$ \\
Luminosity & $11 \%$ \\
\hline total & $17-18 \%$ \\
\hline
\end{tabular}

The inclusive $b$-quark production cross-section in the kinematic range is then calculated by

$$
\sigma\left(p p \rightarrow b+X \rightarrow \mu+X^{\prime}, p_{T}^{\mu}>5 \mathrm{GeV},\left|\eta^{\mu}\right|<2.1\right)=\frac{\alpha_{b} \cdot N_{b, r e c}^{M C}}{\mathcal{L} \cdot \varepsilon}
$$

where $\alpha_{b}$ is the scale factor obtained by the fit that has to be applied to the number of simulated events which pass the event selection $N_{b, r e c}^{M C}, \varepsilon$ is the efficiency of the event selection as determined from simulation and $\mathcal{L}$ is the integrated luminosity. The extrapolation of the measured cross section to the cross section which does not include the definition of the TrackJet amounts to about $10 \%$ and is absorbed in the definition of the efficiency. The inclusive cross section can be measured with a negligible statistical uncertainty and a systematic uncertainty of $17 \%$.

In figure 4.25 the differential $b$-quark production cross-section as a function of the muon transverse momentum is shown. The statistical uncertainty is of the order of $1-2 \%$, the systematic uncertainty of the order of $17-18 \%$ depending on the muon transverse momentum. In table 4.7 the factors for calculating the differential $b$-quark production cross-section as a function of the transverse momentum of the muon are summarized. The results were obtained by dividing the available MC statistics into two independent subsamples using one part to extract the templates and treating the other one as "data".

The result is compared to the leading order and next-to-leading order MC prediction obtained from the PYTHIA and MC@NLO simulation. Since the "data" points correspond to the PYTHIA simulation, they are compatible to the leading order prediction. The MC@NLO distribution lies below the PYHTIA distribution for muon transverse momenta up to about $20 \mathrm{GeV}$. Above this value the two curves agree within the statistical uncertainty.

The differential $b$-quark production cross-section as a function of the muon pseudorapidity is shown in figure 4.26. The result obtained from "data" matches the PYTHIA prediction. The prediction obtained by the MC@NLO simulation is significantly lower. The systematic uncertainty is of the order of $17 \%$ in all bins. The factors for calculating the differential $b$-quark production cross-section as a function of the muon pseudorapidity are given in table 4.8. 
Table 4.7: Differential $b$-quark cross-section $d \sigma / d p_{T}$ for $|\eta|<2.1$ in bins of muon transverse momentum. The table shows the number of selected events in $1 \mathrm{pb}^{-1}$, the $b$-fraction determined by the fit and total efficiency of the event selection for each bin. In the last two columns the calculated differential cross section as a function of the muon transverse momentum and the systematic uncertainty are given.

\begin{tabular}{lrrrrr}
\hline$p_{T}^{\mu}$ & $N_{\text {sel }}$ & $b$-fraction & Total efficiency & $d \sigma / d p_{T}[\mathrm{pb}]$ & Systematic \\
\hline $5-6 \mathrm{GeV}$ & 1535670 & $0.42 \pm 0.002$ & $0.52 \pm 0.001$ & 1229940 & $18 \%$ \\
$6-7 \mathrm{GeV}$ & 876872 & $0.48 \pm 0.002$ & $0.62 \pm 0.002$ & 671684 & $18 \%$ \\
$7-8 \mathrm{GeV}$ & 499809 & $0.54 \pm 0.003$ & $0.69 \pm 0.002$ & 391295 & $18 \%$ \\
$8-10 \mathrm{GeV}$ & 468886 & $0.58 \pm 0.002$ & $0.76 \pm 0.002$ & 178080 & $18 \%$ \\
$10-12 \mathrm{GeV}$ & 181433 & $0.60 \pm 0.003$ & $0.81 \pm 0.003$ & 67310 & $18 \%$ \\
$12-14 \mathrm{GeV}$ & 80323 & $0.67 \pm 0.005$ & $0.86 \pm 0.005$ & 31269 & $17 \%$ \\
$14-16 \mathrm{GeV}$ & 40137 & $0.68 \pm 0.006$ & $0.89 \pm 0.007$ & 15423 & $17 \%$ \\
$16-20 \mathrm{GeV}$ & 30486 & $0.70 \pm 0.006$ & $0.92 \pm 0.007$ & 5835 & $17 \%$ \\
$20-30 \mathrm{GeV}$ & 15413 & $0.72 \pm 0.01$ & $0.90 \pm 0.01$ & 1234 & $17 \%$ \\
$30-50 \mathrm{GeV}$ & 2796 & $0.71 \pm 0.02$ & $0.94 \pm 0.02$ & 106 & $17 \%$ \\
\hline
\end{tabular}

Table 4.8: Differential $b$-quark cross-section $d \sigma / d \eta$ for $p_{T}>5 \mathrm{GeV}$ in bins of muon pseudorapidity. The table shows the number of selected events in $1 \mathrm{pb}^{-1}$, the $b$-fraction determined by the fit and total efficiency of the event selection for each bin. In the last two columns the calculated differential cross section as a function of the muon pseudorapidity and the systematic uncertainty are given.

\begin{tabular}{lrrrrr}
\hline$\eta^{\mu}$ & $N_{\text {sel }}$ & $b$-fraction & Total efficiency & $d \sigma / d \eta[\mathrm{pb}]$ & Systematic \\
\hline$(-2.1,-1.8)$ & 209925 & $0.52 \pm 0.005$ & $0.62 \pm 0.004$ & 593607 & $17 \%$ \\
$(-1.8,-1.5)$ & 244033 & $0.47 \pm 0.004$ & $0.61 \pm 0.004$ & 629892 & $17 \%$ \\
$(-1.5,-1.2)$ & 286894 & $0.48 \pm 0.004$ & $0.65 \pm 0.003$ & 708336 & $17 \%$ \\
$(-1.2,-0.9)$ & 277631 & $0.49 \pm 0.004$ & $0.63 \pm 0.003$ & 716635 & $17 \%$ \\
$(-0.9,-0.6)$ & 289568 & $0.50 \pm 0.004$ & $0.65 \pm 0.003$ & 744271 & $17 \%$ \\
$(-0.6,-0.3)$ & 306464 & $0.50 \pm 0.004$ & $0.66 \pm 0.003$ & 772191 & $17 \%$ \\
$(-0.3,0)$ & 261343 & $0.51 \pm 0.004$ & $0.58 \pm 0.003$ & 760027 & $17 \%$ \\
$(0,0.3)$ & 261173 & $0.53 \pm 0.004$ & $0.58 \pm 0.003$ & 792545 & $17 \%$ \\
$(0.3,0.6)$ & 298028 & $0.51 \pm 0.004$ & $0.65 \pm 0.003$ & 787942 & $17 \%$ \\
$(0.6,0.9)$ & 286553 & $0.45 \pm 0.004$ & $0.63 \pm 0.003$ & 679365 & $17 \%$ \\
$(0.9,1.2)$ & 279678 & $0.47 \pm 0.004$ & $0.61 \pm 0.003$ & 695951 & $17 \%$ \\
$(1.2,1.5)$ & 283733 & $0.46 \pm 0.004$ & $0.64 \pm 0.003$ & 675545 & $17 \%$ \\
$(1.5,1.8)$ & 245298 & $0.47 \pm 0.004$ & $0.62 \pm 0.003$ & 617895 & $17 \%$ \\
$(1.8,2.1)$ & 210679 & $0.47 \pm 0.005$ & $0.62 \pm 0.004$ & 535796 & $17 \%$ \\
\hline
\end{tabular}




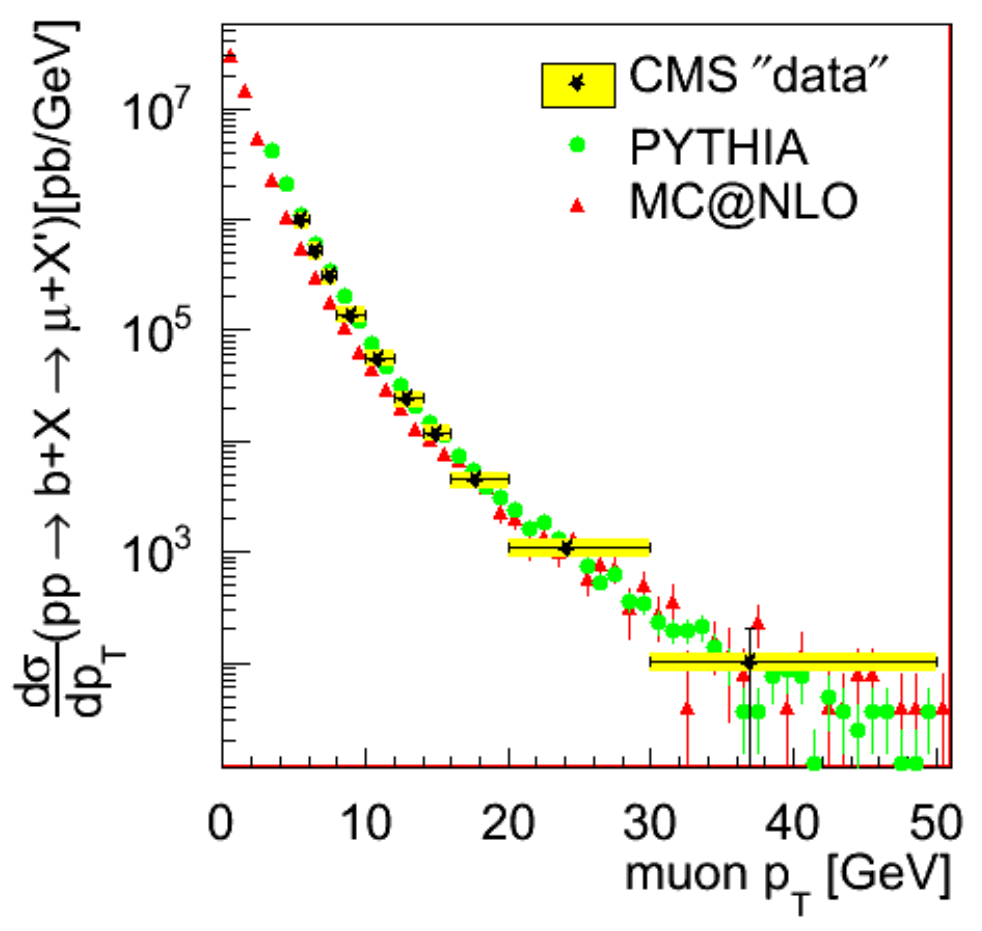

Figure 4.25: Differential $b$-quark production cross-section $d \sigma / d p_{T}$ for $\left|\eta^{\mu}\right|<2.1$ as a function of the muon transverse momentum. The black squares represent the cross section determined by the procedure described in this analysis. The vertical error bars show the statistical uncertainty, the systematic uncertainty is indicated by the yellow area. The horizontal error bars indicated the bin width. The bin center is corrected [89]. The distribution is compared to the prediction of the PYTHIA simulation (green circles) and the MC@NLO simulation (red triangles). 


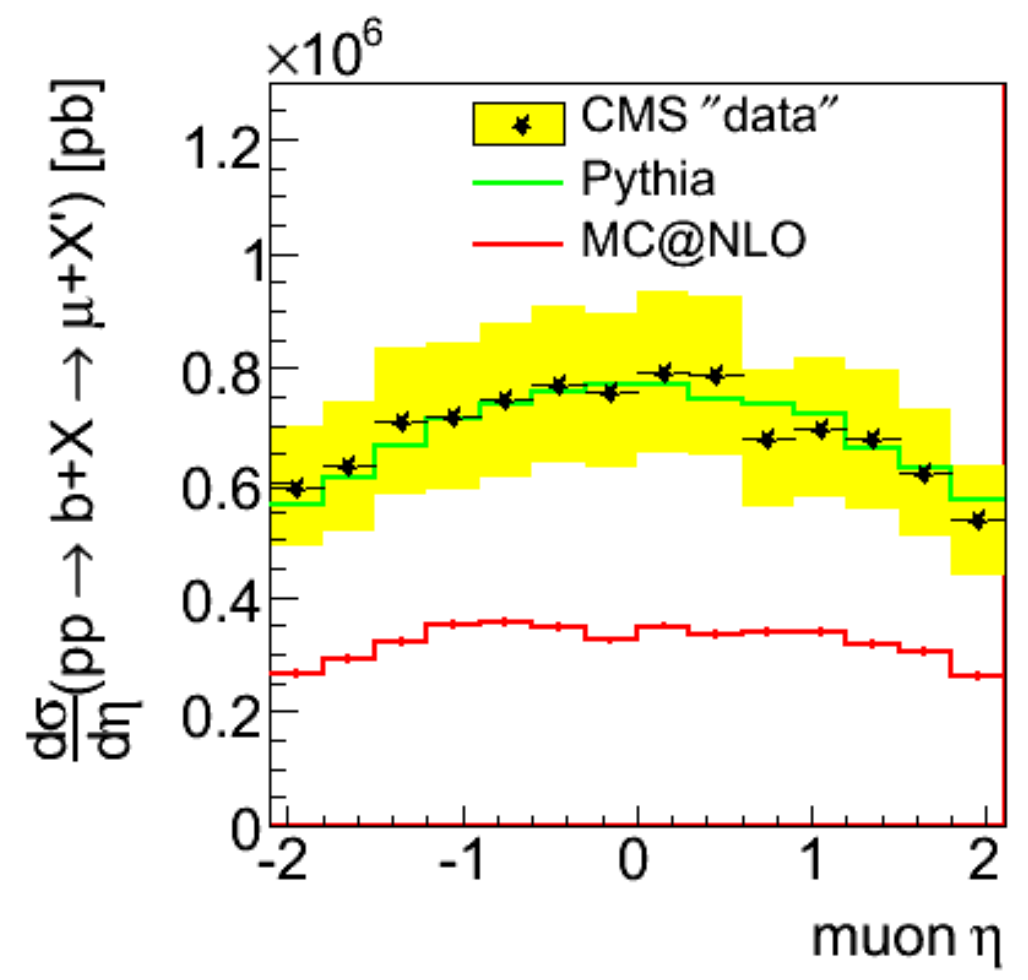

Figure 4.26: Differential $b$-quark production cross-section $d \sigma / d \eta$ for $p_{T}^{\mu}>5 \mathrm{GeV}$ as a function of the muon pseudorapidity. The black dots represent the cross section determined by the procedure described in this analysis. The error bars show the statistical uncertainty, the systematic uncertainty is indicated by the yellow area. The horizontal error bars indicated the bin width. The bin center is corrected [89]. The distribution is compared to the prediction of the PYTHIA simulation (green line) and the MC@NLO simulation (red line). 


\section{Chapter 5}

\section{Results of First Collisions at $\sqrt{s}=900 \mathrm{GeV}$ and $\sqrt{s}=2.36 \mathrm{TeV}$}

In December 2009 proton-proton collisions at the LHC were recorded with the CMS detector for the first time. The collisions happened at a center-of-mass energy of $\sqrt{s}=900 \mathrm{GeV}$ and $\sqrt{s}=2.36 \mathrm{TeV}$. The data collected during the first LHC running period were used in this work to study the performance of the physics object reconstruction and to compare it to the results of the MC simulation.

In the first section the event selection is discussed, before an overview of the event simulation is given. The following sections are devoted to the global muon, track and TrackJet reconstruction. A further section addresses the determination of the $p_{\perp}^{\text {rel }}$ variable in data. The conclusions are given in the last section.

\subsection{Event Selection}

This analysis uses the data collected during runs where the magnetic field was stable at the nominal value of $3.8 \mathrm{~T}$ and the pixel and strip tracking detectors were operational with the high-voltage switched on. Data events corresponding to an integrated luminosity of about $\mathcal{L}=8.5 \mu \mathrm{b}^{-1}$ at a center-of-mass energy of $\sqrt{s}=900 \mathrm{GeV}$ and about $\mathcal{L}=0.5 \mu \mathrm{b}^{-1}$ at $\sqrt{s}=2.36 \mathrm{TeV}$ were recorded during these runs.

Because of the relatively low luminosity provided by the LHC during the first running period, the CMS readout was triggered by signals in two elements of the detector monitoring system [14], namely the beam scintillator counter (BSC) [90] and the beam pick-up timing detector (BPTX) [91]. The BSC detectors consist of 16 scintillator tiles and are located at a distance of $\pm 10.86 \mathrm{~m}$ from the nominal interaction point. They cover a pseudorapidity range of $3.23<|\eta|<4.65$ and have a time resolution of $3 \mathrm{~ns}$. A more precise timing information with a resolution of $0.2 \mathrm{~ns}$ is provided by the BPTX which are located around the beam pipe at a distance of $\pm 175 \mathrm{~m}$.

The passage of the proton bunches in the beam was detected on the basis of the BPTX signals, while the BSC signals were used to collect minimum-bias collision events and reject beam background events. Events were accepted if a signal was seen in coincidence in the two BPTXs and if a time coincidence was also recorded in the BSCs compatible with particles coming from the interaction point and incompatible with beam produced particles crossing from one side to the other. 
In order to further reduce the background from non-collision events, additional event selection were applied in the offline reconstruction. First, the presence of a reconstructed primary vertex in the event was required. Furthermore, beam-induced background events producing an anomalously large number of pixel hits were excluded by rejecting all events with a fraction of high-purity tracks of less than 0.25 in a track multiplicity larger than 10 . The purity of a track is based on the normalized $\chi^{2}$, the longitudinal and transverse impact parameter and their significance [92]. The number of events passing the event selection are listed in table 5.1 .

Table 5.1: Number of data events passing the selection.

\begin{tabular}{lrr}
\hline$\sqrt{s}$ & $N_{\text {sel }}$ & $\mathcal{L}\left[\mu \mathrm{b}^{-1}\right]$ \\
\hline $900 \mathrm{GeV}$ & 278179 & 8.5 \\
$2.36 \mathrm{TeV}$ & 12932 & 0.5 \\
\hline
\end{tabular}

\subsection{Event Simulation}

The MC events were generated with PYTHIA version 6.4 using tune D6T and simulated and reconstructed within the CMS software framework version CMSSW_3_3_6. The event samples consist of inclusive minimum bias events (PYTHIA MSEL=1 card). The corresponding cross sections and luminosities for proton-proton collisions at $\sqrt{s}=900 \mathrm{GeV}$ and $\sqrt{s}=2.36 \mathrm{TeV}$ are given in table 5.2.

The same event selection is applied to MC and data events. The BSC triggers are emulated at the stage of the detector simulation. The number of events which fulfill the event selection criteria are listed in table 5.2.

Table 5.2: Overview of the event simulation. Events were generated using the PYTHIA MSEL=1 card. The center-of-mass energy of the proton-proton collisions, the cross section, the number of generated events and the number of selected events are listed. In the last column the equivalent integrated luminosity is given.

\begin{tabular}{lrrrr}
\hline$\sqrt{s}$ & $\sigma[\mu \mathrm{b}]$ & $N_{\text {gen }}$ & $N_{\text {sel }}$ & $\mathcal{L}\left[\mu \mathrm{b}^{-1}\right]$ \\
\hline $900 \mathrm{GeV}$ & 52410 & 10083360 & 6250310 & 192.4 \\
$2.36 \mathrm{TeV}$ & 59960 & 10654900 & 6726115 & 177.7 \\
\hline
\end{tabular}

\subsection{Muon Distributions}

A lot of progress has been made in the reconstruction of global muons with the CMS detector using the cosmic muon data collected during two extended data taking periods in autumn 2008 and summer 2009. In the collision data 149 global muons were detected at $\sqrt{s}=900 \mathrm{GeV}$ and 15 global muons at $\sqrt{s}=2.36 \mathrm{TeV}$. The muons were reconstructed using the standard procedure described in section 2.2.7 and a minimum transverse momentum of $p_{T}>1 \mathrm{GeV}$ and a pseudorapidity of $-2.5<\eta<2.5$ were required in the offline selection. Basic distributions of global muons are displayed in figure 5.1. There the number of muons per event and the 
transverse momentum, pseudorapidity and azimuthal angle spectrum are shown. The shape of the data distributions are in good agreement with the MC prediction.
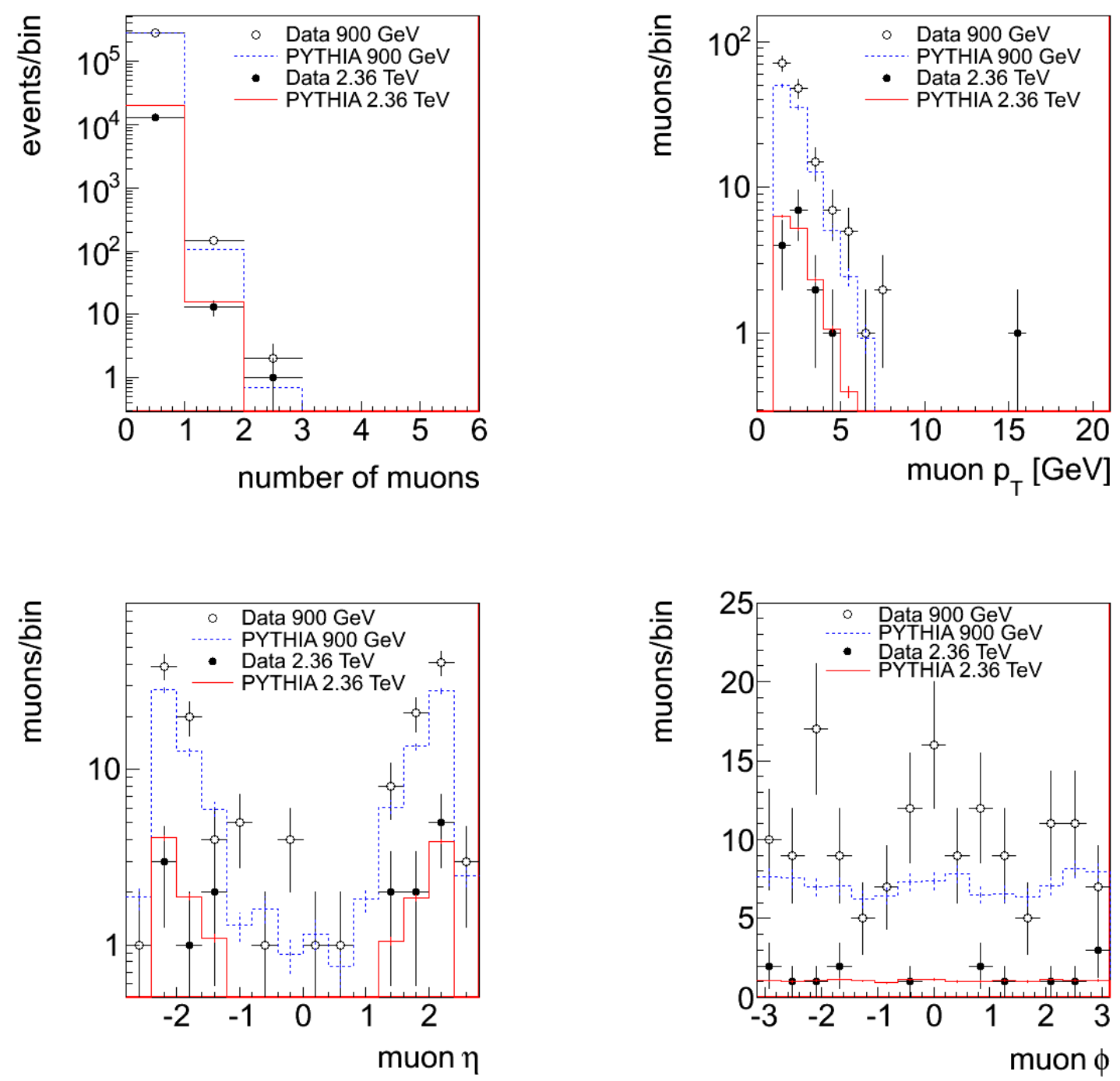

Figure 5.1: Global muon kinematic distributions in data compared to simulation. The MC distributions have been normalized to the data luminosity. The black open circles and the blue dotted line correspond to a center-of-mass energy of $\sqrt{s}=900 \mathrm{GeV}$ and represent the data and MC events, respectively. The events corresponding to a center-of-mass energy of $\sqrt{s}=$ $2.36 \mathrm{TeV}$ are shown as black circles (data) and red lines (MC). Upper left: Number of muons per event. Upper right: Muon transverse momentum. Lower left: Muon pseudorapidity. Lower right: Muon azimuthal angle. 


\subsection{Track Distributions}

For collision data the track reconstruction was performed using the CTF algorithm (see section 2.2.4) and the tracking detectors had been aligned based on the same procedure as described in [94].

Tracks with a minimum transverse momentum of $p_{T}>0.3 \mathrm{GeV}$ and a pseudorapidity of $-2.5<\eta<2.5$ were selected and the quality cuts listed in table 4.4 were applied. The performance of the track reconstruction for the data is compared to the MC simulation. In figure 5.2 the distribution of the track multiplicity and the track transverse momentum, pseudorapidity and azimuthal angle are shown. While the shape of the transverse momentum spectrum in data is well described by MC, the disagreement between data and simulation in track multiplicity and track pseudorapidity is due to an imperfect D6T tuning as discussed in [95]. The asymmetry in the azimuthal angle distribution is due to inactive detector modules affecting mainly low momentum tracks. This has been included in the simulation.

\subsection{TrackJet Distributions}

The tracks selected by the criteria mentioned in the previous section were used as input to the anti- $k_{\mathrm{T}}$ jet clustering algorithm with a cone size of $\mathrm{R}=0.5$. The reconstructed TrackJets are required to have a transverse energy of $E_{T}>1 \mathrm{GeV}$ and a pseudorapidity of $-2.5<\eta<2.5$. An average number of 2.4 TrackJets were reconstructed in the data at $\sqrt{s}=900 \mathrm{GeV}$, whereas the average number of TrackJets at $\sqrt{s}=2.36 \mathrm{TeV}$ is 3.4 . The TrackJet multiplicity is shown in figure 5.3. The inadequate description of the track multiplicity by the PYTHIA D6T tune is reflected in the TrackJet multiplicity distribution. Figure 5.3 also presents the TrackJet transverse energy, pseudorapidity and azimuthal angle distributions. The shape of the transverse momentum is well described by simulation and an average transverse energy of $E_{T}=1.8(1.4) \mathrm{GeV}$ in data (MC) at $\sqrt{s}=900 \mathrm{GeV}$ and $E_{T}=1.9(1.9) \mathrm{GeV}$ at $\sqrt{s}=2.36 \mathrm{TeV}$ is measured.

In figure 5.4 the number of tracks reconstructed within a TrackJet and the transverse momentum of the highest transverse momentum track are compared to the MC simulation and a good agreement is found. These results are relevant in view of the measurement of the $b$-quark production cross-section as the analysis presented here is based on a precise determination of the muon momentum with respect to the TrackJet direction. A good understanding of the TrackJet reconstruction and a reliable simulation of the TrackJet distributions are thus of utmost importance.

Furthermore, the performance of the reconstruction of the transverse impact parameter was investigated. In the measurement of the $b$-quark cross section events with muons with a large transverse impact parameter significance will be used in order to obtain a $b$-enriched sample. Alternatively, it might be possible to use the transverse impact parameter of the tracks in the TrackJet as discriminating variable. In figure 5.5 the transverse impact parameter significance of all tracks in the TrackJet and of the track with the highest transverse impact parameter significance is shown. The impact parameter is calculated with respect to the reconstructed primary vertex and the sign is relative to the axis of the TrackJet. The distributions of the MC simulations are in good agreement with the data distributions. 

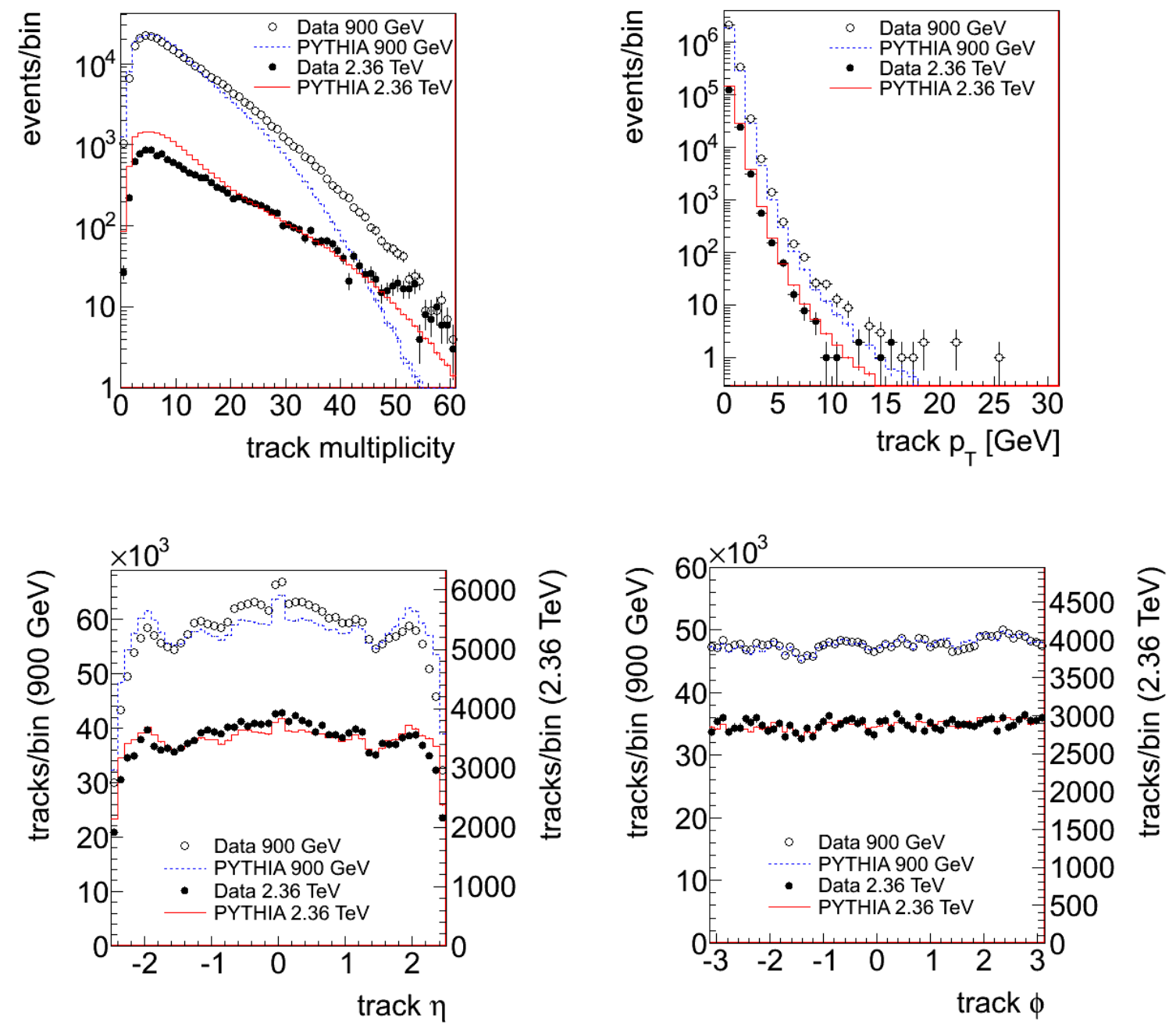

Figure 5.2: Track kinematic distributions in data compared to simulation. The black open circles and the blue dotted line correspond to a center-of-mass energy of $\sqrt{s}=900 \mathrm{GeV}$ and represent the data and MC events, respectively. The events corresponding to a center-ofmass energy of $\sqrt{s}=2.36 \mathrm{TeV}$ are shown as black circles (data) and red lines (MC). Upper left: Number of tracks per event. Upper right: Track transverse momentum. Lower left: Track pseudorapidity. Lower right: Track azimuthal angle. The simulated distribution of the track multiplicity has been normalized to the data luminosity. Since the track multiplicity is different for data and MC events, the simulated distributions of the kinematic variables are scaled to the number of data events in order to allow for a comparison of the shapes. 

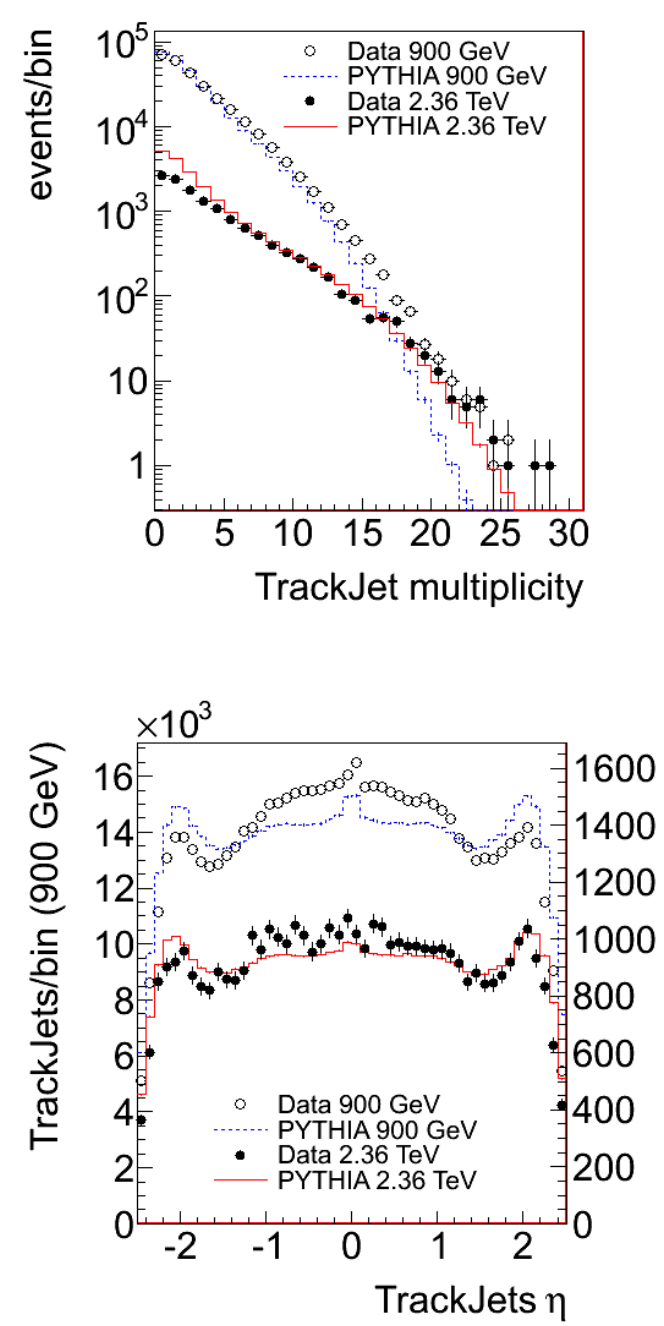
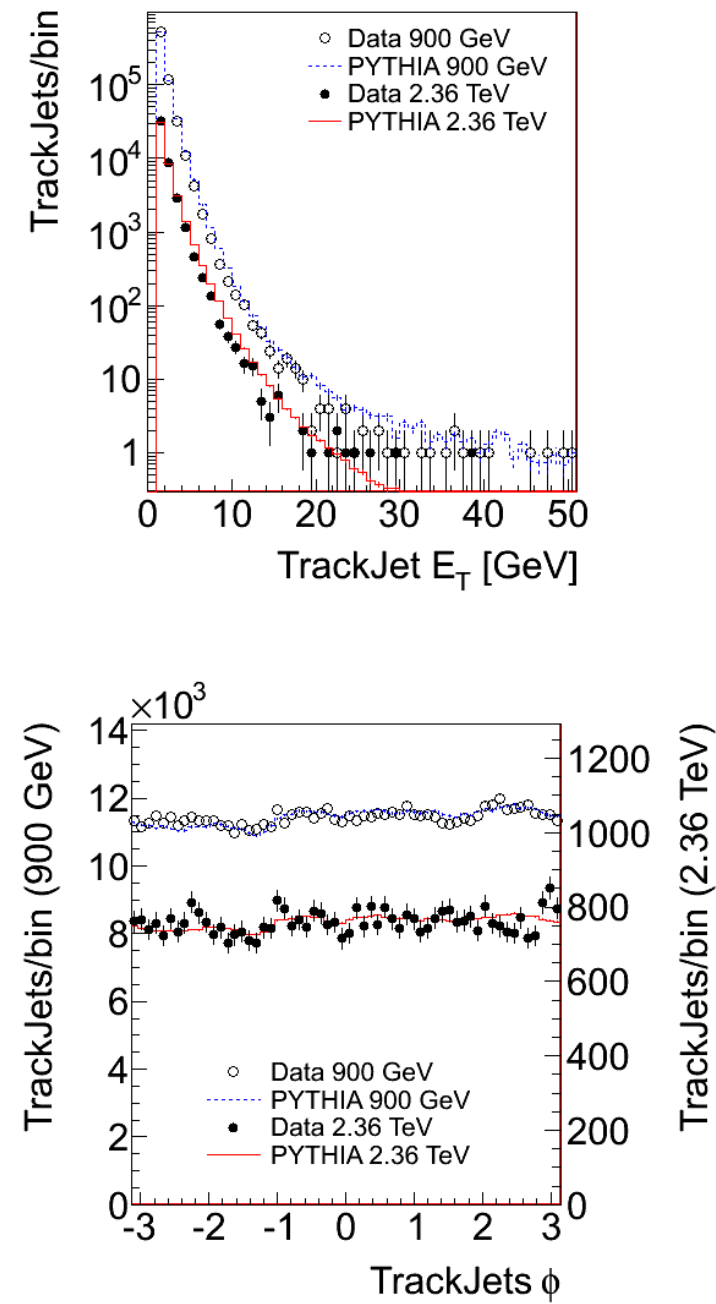

Figure 5.3: TrackJet kinematic distributions in data compared to simulation. The black open circles and the blue dotted line correspond to a center-of-mass energy of $\sqrt{s}=900 \mathrm{GeV}$ and represent the data and MC events, respectively. The events corresponding to a center-of-mass energy of $\sqrt{s}=2.36 \mathrm{TeV}$ are shown as black circles (data) and red lines (MC). Upper left: Number of TrackJets per event. Upper right: TrackJet transverse momentum. Lower left: TrackJet pseudorapidity. Lower right: TrackJet azimuthal angle. The simulated distribution of the TrackJet multiplicity has been normalized to the data luminosity. Since the TrackJet multiplicity is different for data and MC events, the simulated distributions of the kinematic variables are scaled to the number of data events in order to allow for a comparison of the shapes. 

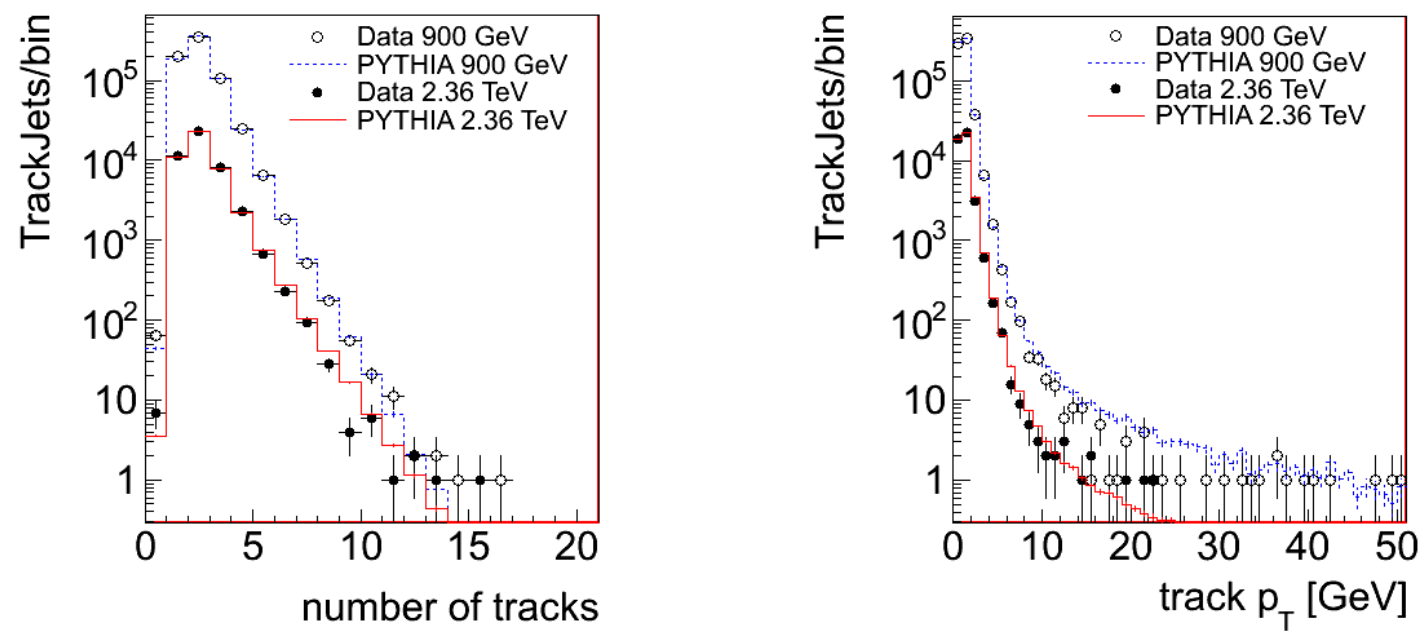

Figure 5.4: Number of tracks per TrackJet (left) and transverse momentum of the highest transverse momentum track in the TrackJets (right). The data distributions are compared to simulation. The simulated distributions have been normalized to the number of TrackJets in data. The black open circles and the blue dotted line correspond to a center-of-mass energy of $\sqrt{s}=900 \mathrm{GeV}$ and represent the data and MC events, respectively. The events corresponding to a center-of-mass energy of $\sqrt{s}=2.36 \mathrm{TeV}$ are shown as black circles (data) and red lines $(\mathrm{MC})$.

\section{6 $\quad p_{\perp}^{\mathrm{rel}}$ Distribution}

According to the PYTHIA simulation, the $b$-quark production cross section is $\sigma_{b}=28 \mu \mathrm{b}$ at $\sqrt{s}=900 \mathrm{GeV}$ and $\sigma_{b}=96 \mu \mathrm{b}$ at $\sqrt{s}=2.36 \mathrm{TeV}$. Thus, the number of events containing $b$-quarks is very low in the 2009 collision data and a measurement of the cross section using the $p_{\perp}^{\text {rel }}$ method will not be possible. Nonetheless it is instructive to study the $p_{\perp}^{\text {rel }}$ distribution in this data in order to better understand the background.

The $p_{\perp}^{\text {rel }}$ distribution in the data at $\sqrt{s}=900 \mathrm{GeV}$ is shown in figure 5.6. The $p_{\perp}^{\text {rel }}$ variable is determined in events with a muon with transverse momentum $p_{T}>3 \mathrm{GeV}$ and pseudorapidity $-2.1<\eta<2.1$ and a TrackJet with transverse momentum $E_{T}>1 \mathrm{GeV}$. In data, 16 events pass this selection, whereas in simulation only 11 events are selected. In the data at $\sqrt{s}=$ $2.36 \mathrm{TeV}$ two events are selected and values of $p_{\perp}^{\mathrm{rel}}=1.45 \mathrm{GeV}$ and $p_{\perp}^{\mathrm{rel}}=1.49 \mathrm{GeV}$ are measured. These numbers are in agreement with the simulation where also 2 events are reconstructed and a mean value of $p_{\perp}^{\text {rel }}=1.36 \mathrm{GeV}$ is found.

The method for validating the background templates by re-weighting the hadronic track spectrum by the muon fake probability (see section 4.7) is investigated. The data statistics is too low to perform a measurement of the muon fake probability and it is therefore taken from simulation. For muons with transverse momentum $p_{T}<5 \mathrm{GeV}$ the fake probability strongly depends on the pseudorapidity as can be seen in figure 5.7. When re-weighting the track spectrum in minimum bias events the dependence of the fake probability on the transverse 

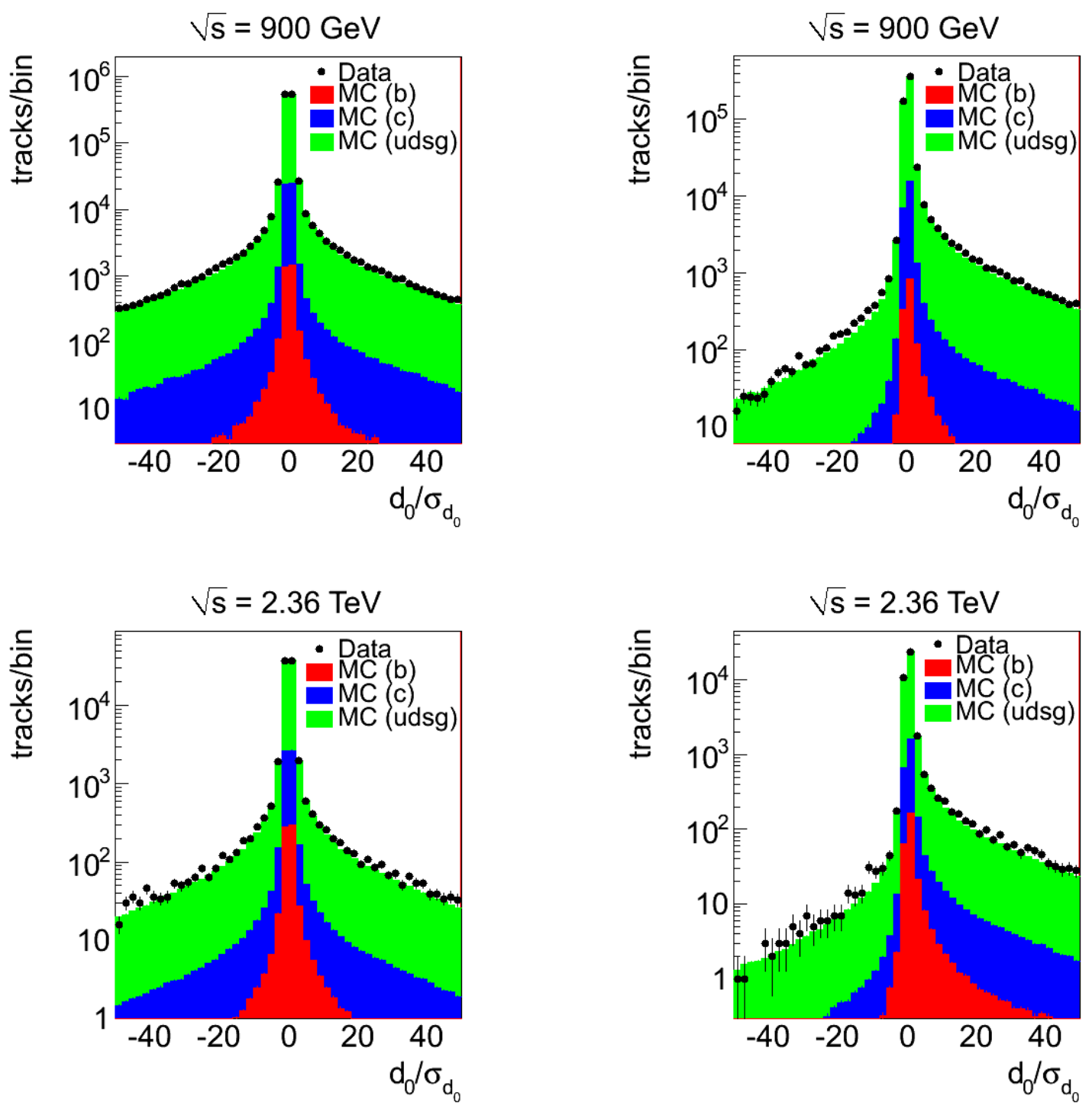

Figure 5.5: Transverse impact parameter significance of all tracks in the TrackJet (left) and of the track with the highest transverse impact parameter in the TrackJet (right). The upper plots correspond to a center-of-mass energy of $\sqrt{s}=900 \mathrm{GeV}$, the lower plots to $\sqrt{s}=2.36 \mathrm{TeV}$. The simulated distributions are normalized to the number of tracks in data. The black circles represent the data distribution. The MC distributions are split into the contribution of $b$ (red), $c$ (blue) and $u d s g$ (green) events.

momentum and the pseudorapidity is taken into account. The result is shown in figure 5.8.

A harder $p_{\perp}^{\text {rel }}$ distribution is observed in the re-weighted data with respect to simulation. In order to validate the weighting procedure, it was applied to the simulated track spectrum. The results from simulation agree within the statistical error. For comparison, the measured $p_{\perp}^{\mathrm{rel}}$ distribution calculated from muons which were most likely generated in light quark or 
charm decays is also shown in figure 5.8 .

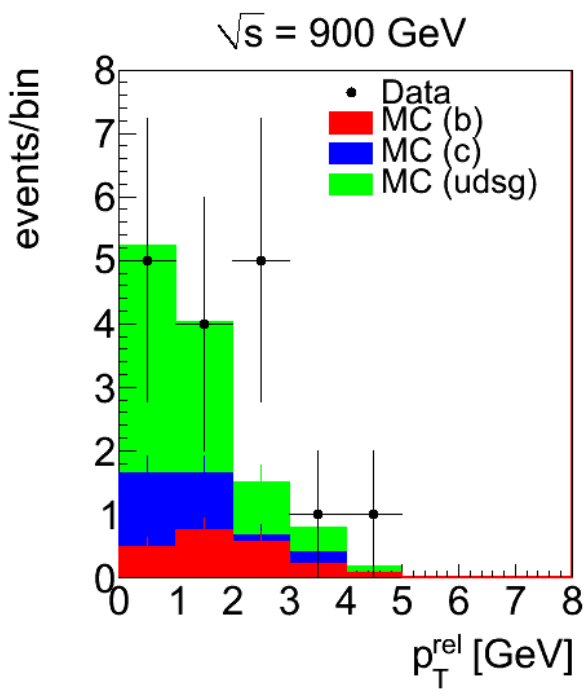

Figure 5.6: $p_{\perp}^{\text {rel }}$ distribution in the collision data at $\sqrt{s}=900 \mathrm{GeV}$ compared to the MC simulation. The black circles represent the data distribution. The MC distributions are divided into the contribution of $b$ (red), $c$ (blue) and $u d s g$ (green) events.
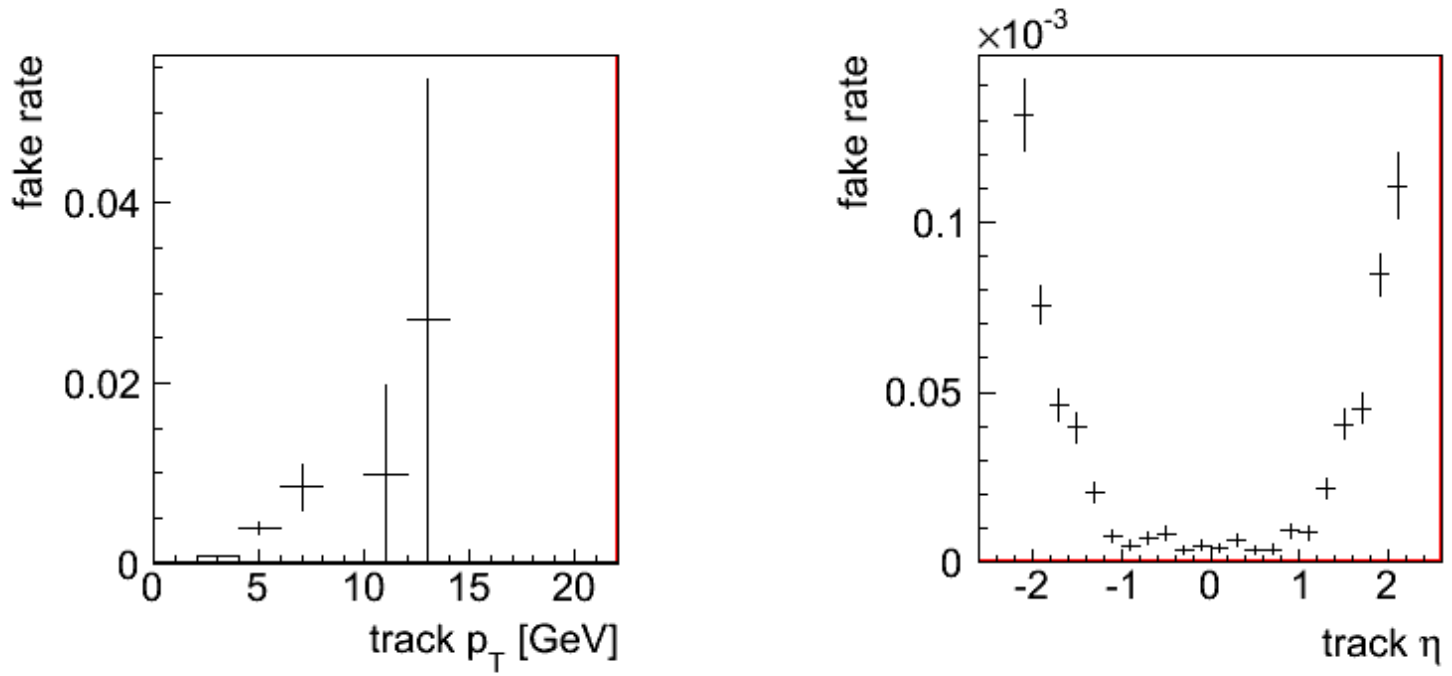

Figure 5.7: Muon fake probability as a function of transverse momentum (left) and pseudorapidity (right) as obtained from simulation. 


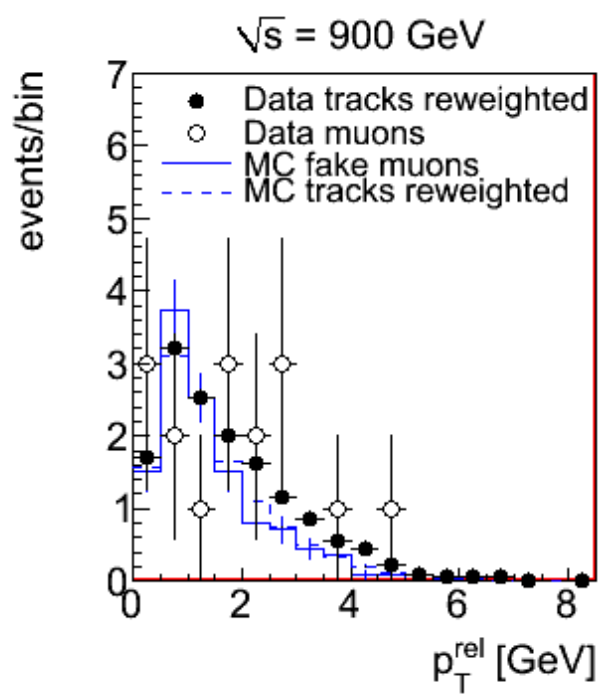

Figure 5.8: $p_{\perp}^{\text {rel }}$ distribution obtained by re-weighting the hadronic track spectrum in data by the simulated muon fake probability (black circles). The result is compared to the simulated fake muon spectrum (blue solid line), the re-weighted hadronic track spectrum in MC (blue dotted line) and the measured $p_{\perp}^{\text {rel }}$ distribution in data (black open circles). The MC distributions are scaled to the data luminosity.

\subsection{Conclusions}

The data recorded during the first collisions in December 2009 have been used to study the performance of the physics object reconstruction. The number of global muons in the collision data is very small. However, the reconstruction of global muons was already well commissioned using cosmic data. The reconstruction of tracks and TrackJet was studied and a in general the data were found to be well described by simulation. This result is most valuable in view of the analysis presented in this thesis.

The $p_{\perp}^{\text {rel }}$ distribution obtained in the data corresponding to $\sqrt{s}=900 \mathrm{GeV}$ was determined and compared to the simulation. The MC simulation is in agreement with data although the statistics is very limited. Furthermore, the method for obtaining the light-quark background $p_{\perp}^{\text {rel }}$ templates using a data-driven approach was validated. 


\section{Chapter 6}

\section{Preliminary Results of First Collisions at $\sqrt{s}=7 \mathrm{TeV}$}

On March 30, 2010, the first proton-proton collisions at a center-of-mass energy of $\sqrt{s}=7 \mathrm{TeV}$ happened at the LHC. The data statistics recorded by the CMS detector during the first months of data-taking allows for a first measurement of the inclusive $b$-quark production crosssection at the LHC [96]. The preliminary result has been presented at the $35^{\text {th }}$ International Conference on High Energy Physics [97]

In this chapter the analysis of the collision data at $\sqrt{s}=7 \mathrm{TeV}$ collected in April and May, 2010 is summarized. In the first section an overview of the event simulation is given, followed by a short discussion of the event selection and the signal extraction. The preliminary results together with the main systematic uncertainties are summarized and discussed in the last two sections.

\subsection{Event Simulation}

The CMS data are compared to the PYTHIA MC simulation version 6.4 with tune D6T. For simulation and reconstruction the CMS software version CMSSW_3_5_X was used. Two statistically independent samples were generated: an inclusive QCD minimum bias sample and a muon-enriched QCD sample, in which the presence of a generated muon with $p_{T}>2.5 \mathrm{GeV}$ and $|\eta|<2.5$ was required (compare to section 4.2). An overview of the MC simulation is given in table 6.1 .

Table 6.1: Overview of the PYTHIA event simulation at $\sqrt{s}=7 \mathrm{TeV}$. An inclusive QCD minimum bias sample and a muon-enriched QCD sample were generated. The center-of-mass energy of the proton-proton collisions, the cross section, the filter efficiency of the generator level filter and the number of selected events are listed in the table. In the last column the equivalent integrated luminosity is given.

\begin{tabular}{lccrrr}
\hline Data set & $\sqrt{s}$ & $\sigma[\mu \mathrm{b}]$ & $\epsilon_{\text {filt }}$ & $N_{\text {reco }}$ & $\mathcal{L}\left[\mathrm{nb}^{-1}\right]$ \\
\hline inclusive QCD & $7 \mathrm{TeV}$ & 71260 & 1 & 10998457 & 0.15 \\
muon enriched QCD & $7 \mathrm{TeV}$ & 48440 & 0.0018 & 10418911 & 119.5 \\
\hline
\end{tabular}




\subsection{Event Selection}

The event selection detailed in section 4.5 is applied to the CMS data and the MC simulation.

\subsubsection{Run Selection}

The data recorded during the first months of CMS data taking at $\sqrt{s}=7 \mathrm{TeV}$ between March 30 and May 19, 2010 were used for this analysis. The integrated luminosity as a function of time is shown in figure 6.1.

CMS: Integrated Luminosity 2010

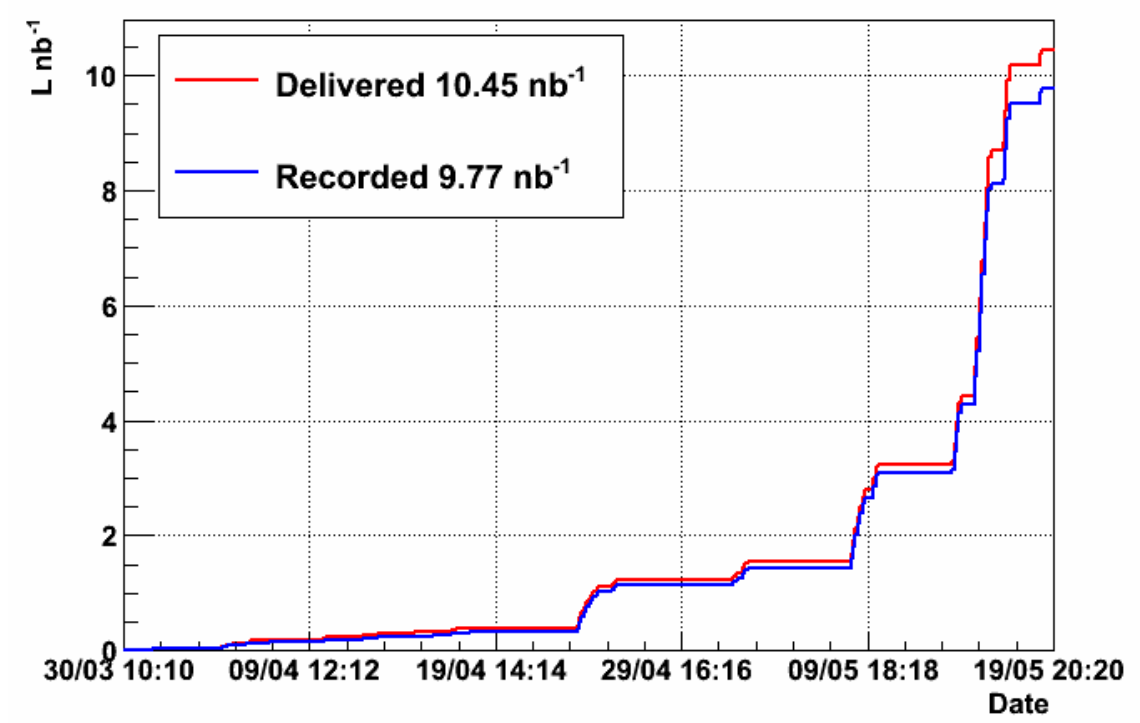

Figure 6.1: Integrated luminosity delivered by the LHC (red line) and recorded by CMS (blue line) during the first data taking at $\sqrt{s}=7 \mathrm{TeV}$ in April and May, 2010.

In the analysis only runs certified by the Data Quality Monitoring group were considered. The run selection is based on the following criteria:

- Stable beam conditions with beam energy at $E=3.5 \mathrm{TeV}$

- Stable magnetic field inside CMS with $B_{z}>3.7 \mathrm{~T}$

- Goodness of L1 trigger and HLT

- Silicon pixel and strip tracking detectors in readout with nominal high-voltage settings

- DT, CSC and RPC muon detectors in readout with nominal high-voltage settings

The data recorded during the runs fulfilling the quality criteria correspond to an integrated luminosity of $\mathcal{L}=8.1 \mathrm{nb}^{-1}$. 


\subsubsection{Trigger Selection}

The events of interest are selected by the HLT_Mu3 single muon trigger path (see section 4.3). The trigger efficiency is measured from data in minimum bias events. The minimum bias events were obtained as described in section 5.1 and the HLT_Mu3 efficiency is calculated by

$$
\varepsilon_{H L T}=\frac{N_{\text {reco }, H L T}^{\mu}}{N_{\text {reco }}^{\mu}},
$$

where $N_{\text {reco }}^{\mu}$ is the number of reconstructed global muons with transverse momentum $p_{T}>$ $6 \mathrm{GeV}$ and pseudorapidity $|\eta|<2.1 . N_{\text {reco }, H L T}^{\mu}$ is the number of reconstructed global muons in the same kinematic range and accepted by the HLT.

The trigger efficiency as a function of muon transverse momentum and pseudorapidity is shown in figure 6.2. It should be noted that the MC simulation overestimates the trigger efficiency.
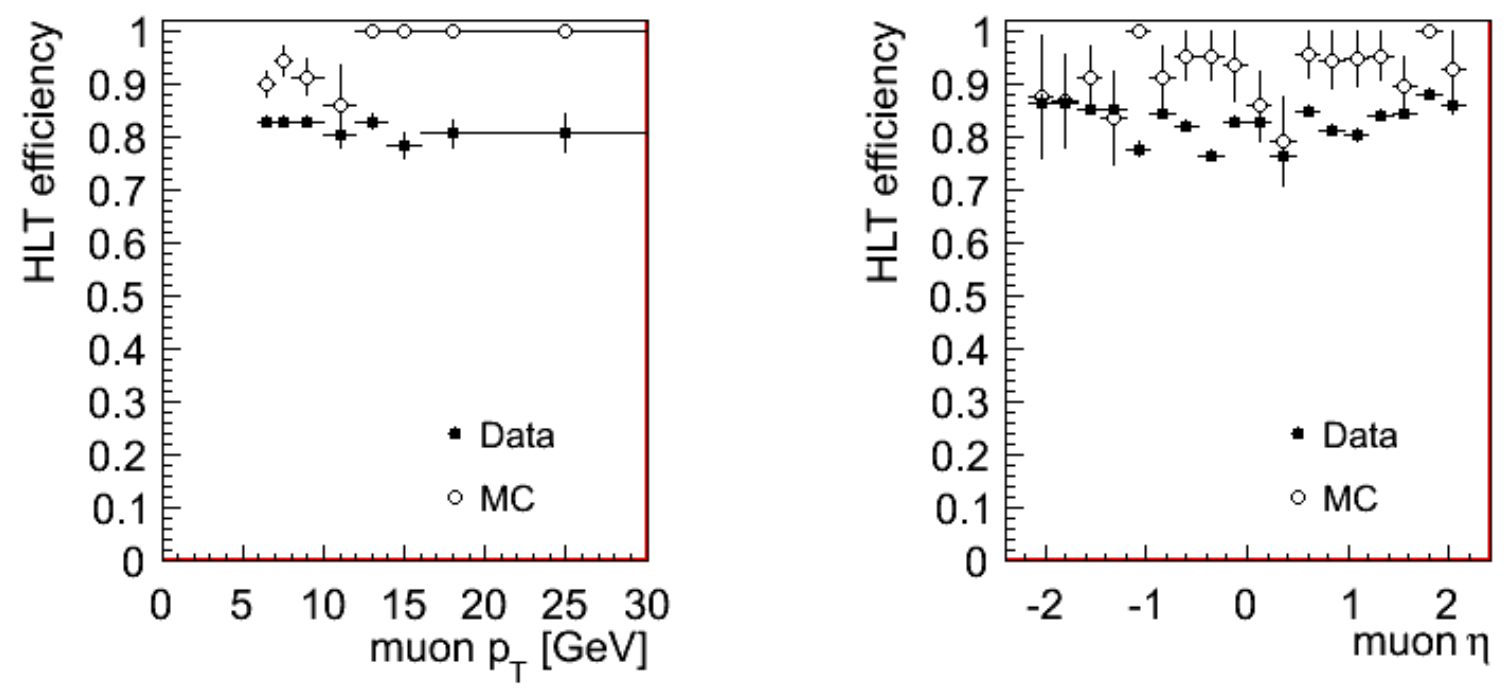

Figure 6.2: Single muon trigger efficiency (HLT_Mu3) as measured from data compared to simulation. The simulated trigger efficiency is determined on the basis of inclusive QCD MC events. Left: HLT efficiency as a function of the muon transverse momentum for muons with pseudorapidity $|\eta|<2.1$. Right: HLT efficiency as a function of muon pseudorapidity for muons with transverse momentum $p_{T}>6 \mathrm{GeV}$.

\subsubsection{Offline Selection}

Background from non-collision events is reduced by requiring a reconstructed primary vertex with more than three tracks while beam-induced background events were rejected if the fraction of high-purity tracks to all tracks was less than 0.25 .

Events with a reconstructed global muon with transverse momentum $p_{T}>6 \mathrm{GeV}$ and pseudorapidity $|\eta|<2.1$ are selected. In order to improve the background rejection, quality 
criteria on the number of hits in the tracker and muon detector, the $\chi^{2}$ of the track fit and the impact parameter are imposed. The selection criteria are listed in table 6.2. In figure 6.3 the transverse momentum, pseudorapidity, azimuthal angle and transverse impact parameter distributions for selected muons in data and simulation are shown. The data distributions are compared to the muon-enriched QCD MC sample. The MC distribution are normalized to the data luminosity and the simulated trigger efficiency is corrected with respect to the trigger efficiency measured in data. The shape of the muon transverse momentum and azimuthal angle distribution are well described by $\mathrm{MC}$ whereas in the pseudorapidity distribution discrepancies between data and $\mathrm{MC}$ are observed.

Table 6.2: Selection criteria for the reconstructed global muons in the offline analysis.

\begin{tabular}{ll}
\hline Variable & Value \\
\hline minimum transverse momentum & $p_{T}>6 \mathrm{GeV}$ \\
maximum pseudorapidity & $|\eta|<2.1$ \\
longitudinal impact parameter & $z_{0}<20 \mathrm{~cm}$ \\
transverse impact parameter & $d_{0}<5 \mathrm{~cm}$ \\
number of pixel layers with hits & $\geqslant 2$ \\
number of valid hits in tracker & $\geqslant 12$ \\
number of valid hits in muon chambers & $>0$ \\
normalized $\chi^{2}$ of tracker track & $<10$ \\
normalized $\chi^{2}$ of global track & $<10$ \\
\hline
\end{tabular}

The $b$-jet is defined as the TrackJet containing the muon. After subtracting the muon momentum from the TrackJet momentum, the TrackJet energy is required to fulfill $E_{T}>$ $1 \mathrm{GeV}$. The probability of associating a TrackJet to the reconstructed muon is $77.4 \pm 0.3 \%$ and $80.8 \pm 0.1 \%$ in data and simulation, respectively. A total of 16826 data events pass the selection.

The distribution of the TrackJet kinematic variables are compared to simulation and the result is displayed in figure 6.4. In general, the data distribution are well described by simulation. The TrackJet pseudorapidity distribution is more central since it is correlated with the pseudorapidity distribution of the muon which also shows this feature. The disagreement between data and MC in the TrackJet transverse energy distribution at low transverse energy is due to the imperfect PYTHIA D6T tune which does not describe correctly the track multiplicity and the track transverse momentum spectrum for low transverse momentum tracks [98].

\subsection{Signal Extraction}

\subsubsection{Data-driven Determination of Light Quark Background}

The light quark background template is obtained from data using the method introduced in section 4.7. Hadrons satisfying all muon track selection criteria (except for muon identification) are re-weighed with the muon fake probability and used instead of muons to determine the $p_{\perp}^{\text {rel }}$ template. The muon fake probability is taken from MC simulation, as the current data sample size does not allow a precise determination of this quantity. 

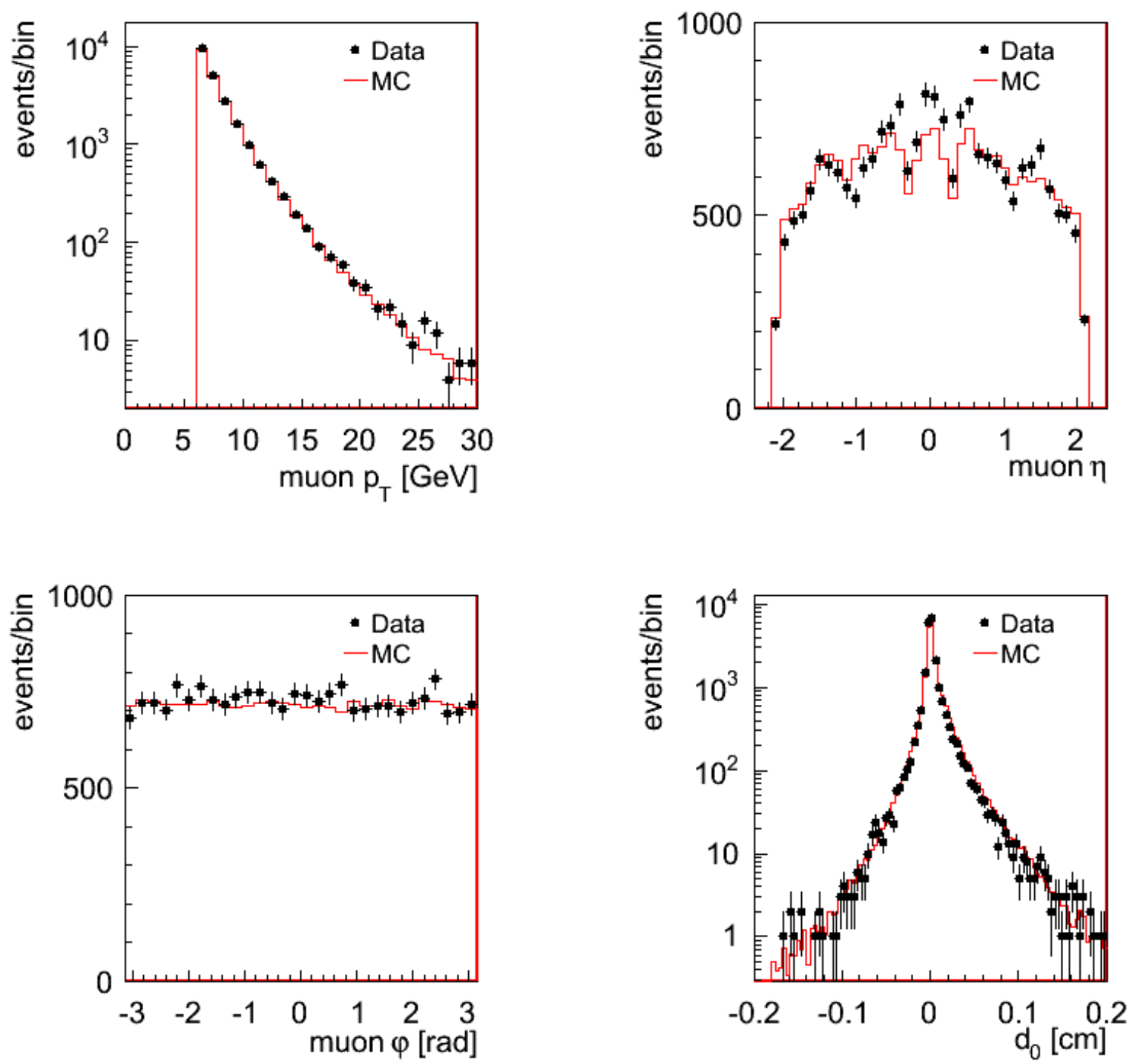

Figure 6.3: Transverse momentum (top left), pseudorapidity (top right), azimuthal angle (lower left) and transverse impact parameter (lower right) distribution of selected reconstructed global muons. The data distribution (black circles) is compared to the MC simulation (red solid line) normalized to the integrated luminosity.

The $p_{\perp}^{\text {rel }}$ distribution of re-weighted tracks in minimum bias events is compared to the one of simulated muons in light quark events in figure 6.5. The udsg template determined from data is harder than in simulation. In order to evaluate the systematic uncertainty of the cross-section measurement due to an imperfect background description, the $p_{\perp}^{\text {rel }}$ distribution obtained from data as well as from simulation is used in the fitting procedure. 

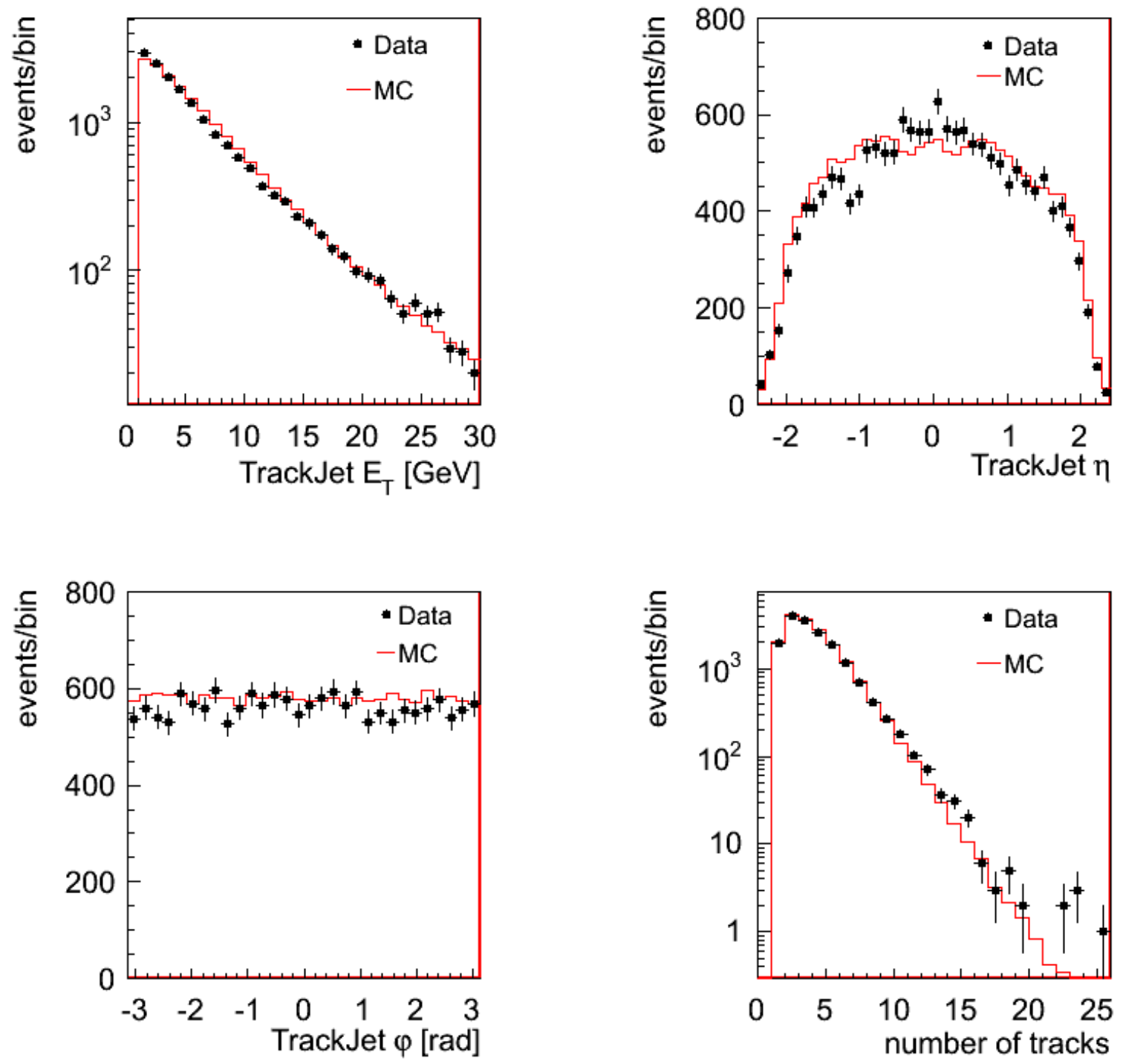

Figure 6.4: Transverse energy (top left), pseudorapidity (top right), azimuthal angle (lower left) and number of tracks (lower right) of selected TrackJets. The data distribution (black circles) is compared to the MC simulation (red solid line) normalized to the integrated luminosity.

\subsubsection{Data-driven Validation of $p_{\perp}^{\text {rel }}$ Templates in Signal Events}

The shape of the signal $p_{\perp}^{\text {rel }}$ distribution is validated using a data-driven method (see section 4.7). A data sample with a high $b$-content is used to cross-check the shape of the $p_{\perp}^{\mathrm{rel}}$ distribution. Such a sample is obtained by selecting events containing single muons with a large impact parameter. By selecting events with $d_{0} / \sigma_{d_{0}}>20$ a $b$-purity about $90 \%$ is achieved. The shape of the $p_{\perp}^{\text {rel }}$ distribution extracted from data events and MC events $(b$ and 


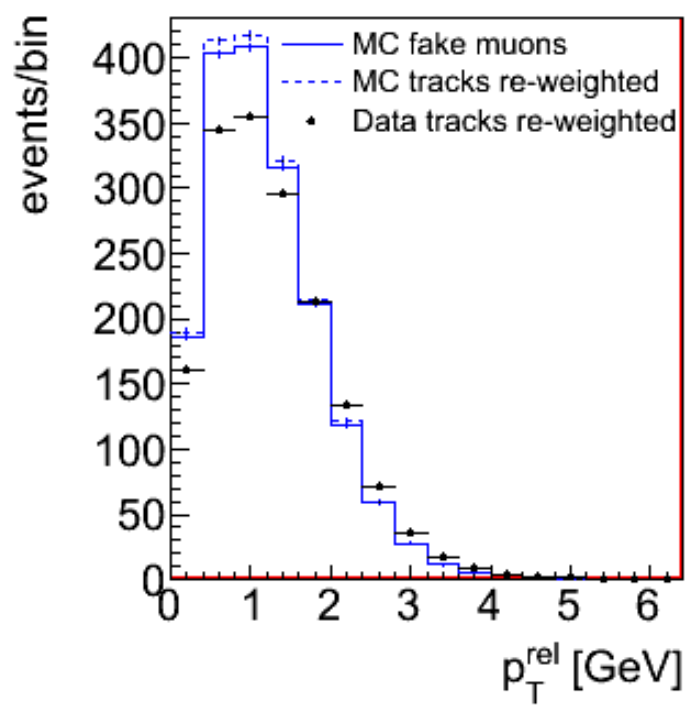

Figure 6.5: $p_{\perp}^{\text {rel }}$ distribution in light quark background events obtained by re-weighting the hadronic track spectrum in data by the simulated muon fake probability (black circles). The result is compared to the simulated fake muon spectrum (blue solid line) and the reweighted hadronic track spectrum in simulation (blue dotted line). The MC distributions are normalized to the number of data events.

inclusive) with a cut on the muon signed transverse impact parameter significance are shown for the inclusive sample in figure 6.6.

No systematic uncertainty is introduced as the $p_{\perp}^{\text {rel }}$ distribution in signal events obtained from simulation agrees with the data distribution within the limited statistics.

\subsection{3 $p_{\perp}^{\mathrm{rel}}$ Fit}

The fitting procedure, discussed in detail in section 4.6, is carried out to determine the fraction of $b$-events among the selected signal events. A binned maximum likelihood to the observed $p_{\perp}^{\text {rel }}$ distribution based on templates obtained from simulation (signal and part of the background) and data (the remaining background) is performed. The fitting procedure is applied to the $p_{\perp}^{\mathrm{rel}}$ spectrum in the inclusive sample, 8 bins in muon transverse momentum and 7 bins in muon pseudorapidity. The result of the fit in the inclusive sample is displayed in figure 6.7, while the results in bins of muon transverse momentum and pseudorapidity are shown in appendix $\mathrm{C}$.

In table 6.3 the $b$-fractions determined by the fit in the different bins and their statistical uncertainty are listed. Furthermore, the likelihood ratio $\chi^{2}$ of the fits is given where the number of degrees of freedom of all fits is 10 .

The stability of the fit is tested by performing repeated fits with varied binning. The results agree within the statistical error. 


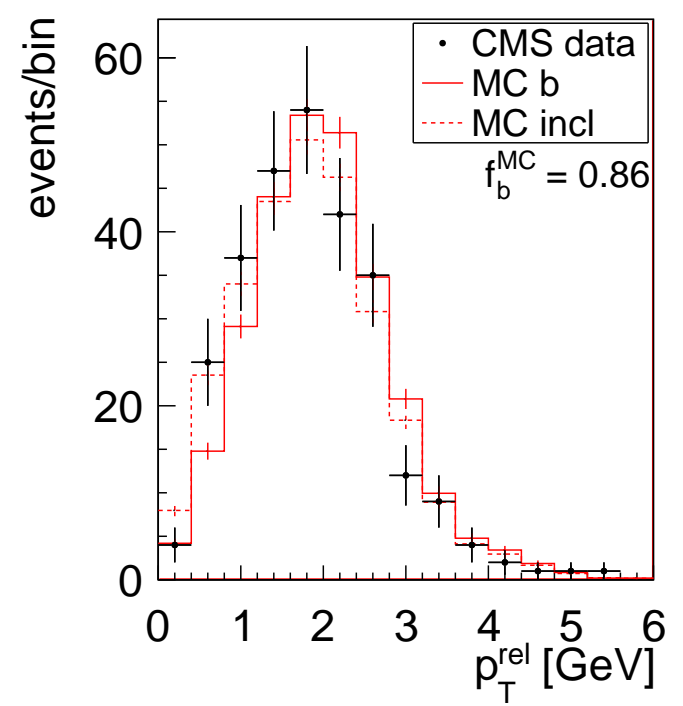

Figure 6.6: $p_{\perp}^{\text {rel }}$ distribution in $b$-enriched events in data and MC obtained by a cut on the muon signed transverse impact parameter significance $d_{0} / \sigma_{d_{0}}>20$. The black circles are the data events, the red solid line corresponds to the $b$-events in MC and the red dotted line is the inclusive QCD MC. The distribution are normalized to the number of data events.

\subsection{Results}

The inclusive $b$-quark production cross-section is calculated according to equation (4.11):

$$
\sigma^{v i s} \equiv \sigma\left(p p \rightarrow b+X \rightarrow \mu+X^{\prime}, p_{T}^{\mu}>6 \mathrm{GeV},\left|\eta^{\mu}\right|<2.1\right)=\frac{\alpha_{b} \cdot N_{b, r e c}^{M C}}{\mathcal{L} \cdot \varepsilon} .
$$

The efficiency $\varepsilon$ includes the trigger efficiency (82\%), the muon reconstruction efficiency $(97 \%)$, and the efficiency for associating a TrackJet to the reconstructed muon (77\%). The trigger efficiency is determined from data, the other two efficiencies are taken from MC simulation.

The preliminary result of the inclusive $b$-quark production cross-section within the kinematical range is

$\sigma\left(p p \rightarrow b+X \rightarrow \mu+X^{\prime}, p_{T}^{\mu}>6 \mathrm{GeV},\left|\eta^{\mu}\right|<2.1\right)=\left(1.48 \pm 0.04_{\text {stat }} \pm 0.22_{\text {syst }} \pm 0.16_{\text {lumi }}\right) \mu \mathrm{b}$.

where the error represents the statistical error of the fit. The systematic error is discussed in the following section.

For comparison, the inclusive $b$-quark production cross section predicted by the PYTHIA and MC@NLO is given:

$$
\begin{aligned}
\sigma_{\mathrm{PYTHIA}}^{\text {vis }} & =1.8 \mu \mathrm{b} \\
\sigma_{\text {MC@NLO }}^{\text {vis }} & =\left[0.84_{-0.19}^{+0.36}(\text { scale }) \pm 0.08\left(m_{b}\right) \pm 0.04(\text { pdf })\right] \mu \mathrm{b} .
\end{aligned}
$$

The error for MC@NLO is obtained by changing the QCD renormalization and factorization scales independently from half to twice their default values within a fiducial volume as 
Table 6.3: $b$-fraction determined by the fit for the inclusive sample and the bins in muon transverse momentum and pseudorapidity. The errors represent the statistical uncertainty. In addition, the likelihood-ratio $\chi^{2}$ of the fit is given. The number of degrees of freedom in the fit is 10 .

\begin{tabular}{lrr}
\hline & Fitted $b$-fraction & $\chi^{2}$ \\
\hline inclusive & & \\
\hline & $0.44 \pm 0.01$ & 28.1 \\
\hline$p_{T}^{\mu}$ & & \\
\hline $6-7 \mathrm{GeV}$ & $0.42 \pm 0.02$ & 9.1 \\
$7-8 \mathrm{GeV}$ & $0.39 \pm 0.03$ & 5.2 \\
$8-10 \mathrm{GeV}$ & $0.48 \pm 0.03$ & 23.0 \\
$10-12 \mathrm{GeV}$ & $0.57 \pm 0.04$ & 12.9 \\
$12-14 \mathrm{GeV}$ & $0.45 \pm 0.06$ & 8.8 \\
$14-16 \mathrm{GeV}$ & $0.45 \pm 0.09$ & 6.6 \\
$16-20 \mathrm{GeV}$ & $0.54 \pm 0.10$ & 6.5 \\
$20-30 \mathrm{GeV}$ & $0.78 \pm 0.15$ & 5.6 \\
\hline$\eta^{\mu}$ & & \\
\hline$(-2.1,-1.5)$ & $0.42 \pm 0.04$ & 11.2 \\
$(-1.5,-0.9)$ & $0.40 \pm 0.03$ & 6.1 \\
$(-0.9,-0.3)$ & $0.46 \pm 0.03$ & 5.4 \\
$(-0.3,0.3)$ & $0.44 \pm 0.03$ & 17.4 \\
$(0.3,0.9)$ & $0.47 \pm 0.03$ & 18.1 \\
$(0.9,1.5)$ & $0.49 \pm 0.03$ & 6.8 \\
$(1.5,2.1)$ & $0.43 \pm 0.04$ & 17.4 \\
\hline
\end{tabular}

in [99]. The massive HERWIG calculation agrees with the MC@NLO prediction within the theorectical uncertainties.

The preliminary results of the differential $b$-quark production cross-section as a function of muon transverse momentum and pseudorapidity are shown in figure 6.8 and 6.9 and summarized in table 6.4 and 6.5 . 


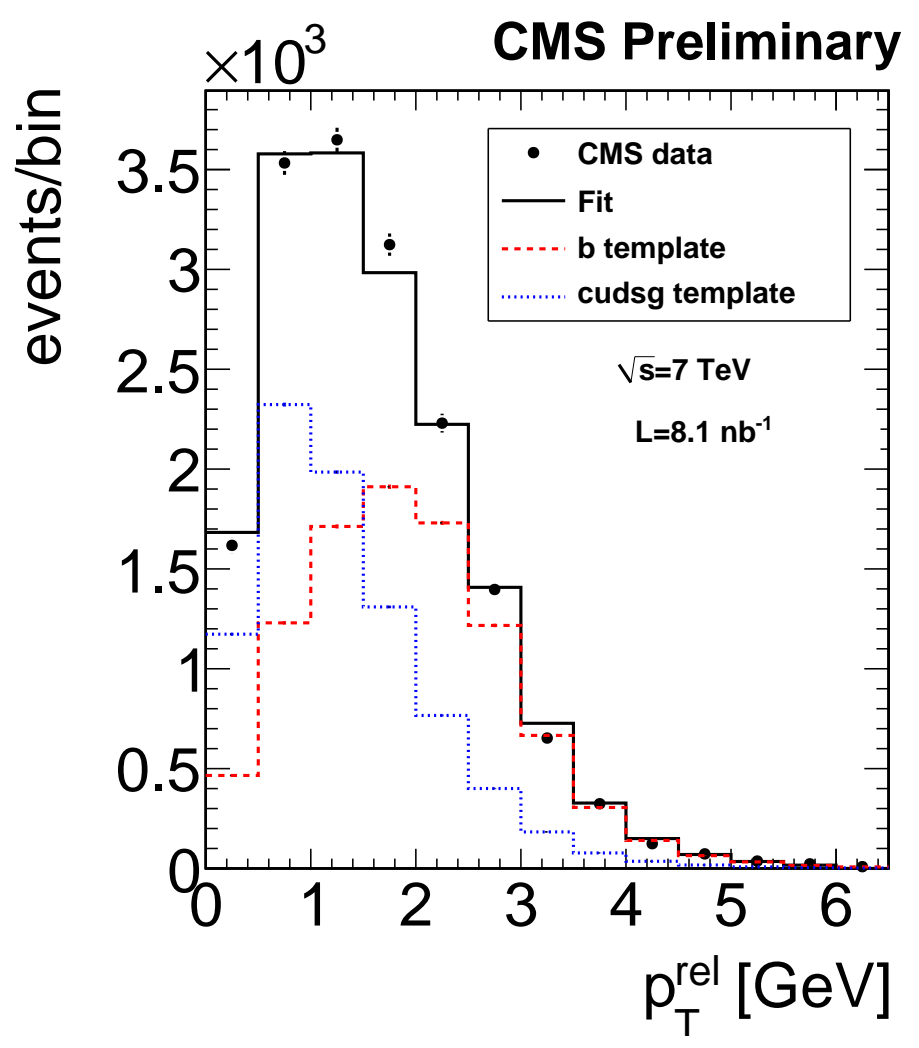

Figure 6.7: $p_{\perp}^{\text {rel }}$ distribution measured in data. Overlaid are the result of the maximum likelihood fit and the simulated template distributions. The red dashed and the blue dotted line are the $b$ - and $c u d s g$-template, respectively. The black full circles correspond to the data distribution, while the black line is the result of the fitting procedure. 
CMS Preliminary

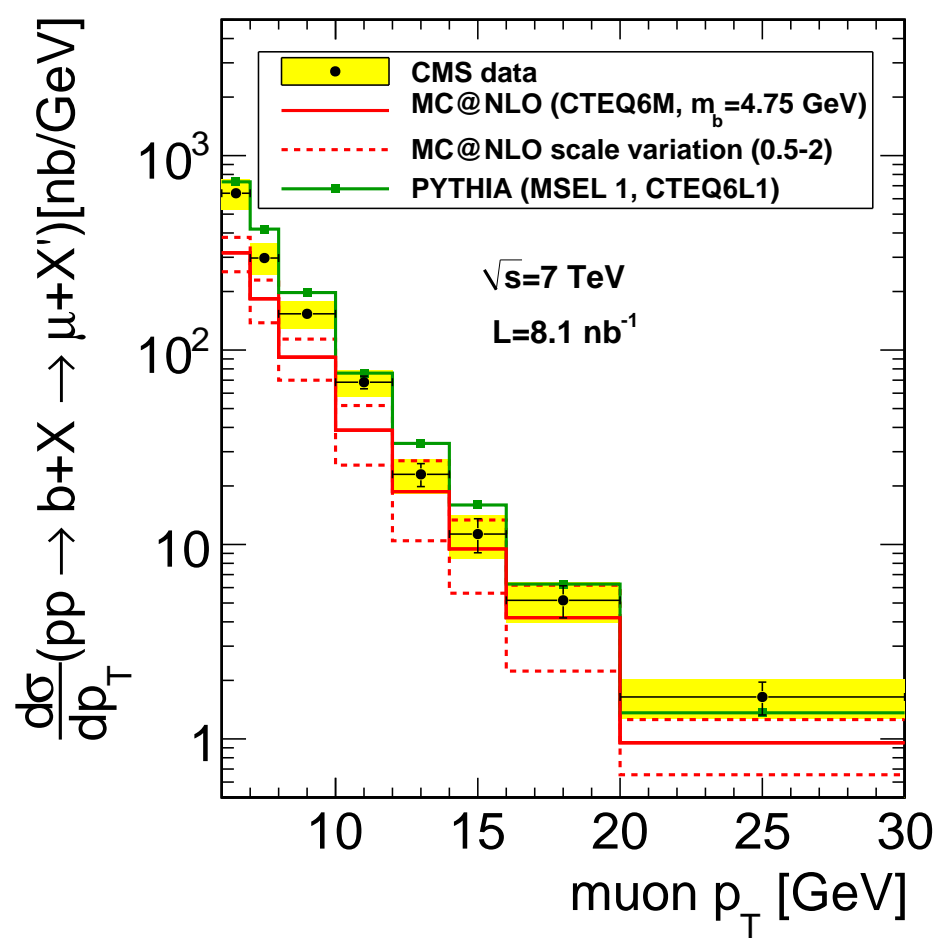

Figure 6.8: Differential $b$-quark production cross-section $d \sigma / d p_{T}$ for $\left|\eta^{\mu}\right|<2.1$ as a function of the muon transverse momentum. The black symbols show the measured cross section where the vertical error bars represent the statistical uncertainty and the horizontal error bars indicate the bin width. The yellow band shows the quadratic sum of statistical and systematic errors. The systematic error (11\%) of the luminosity measurement is not included. The dashed red lines illustrate the MC@NLO theoretical uncertainty as described in the text. The solid green line shows the PYTHIA result. 


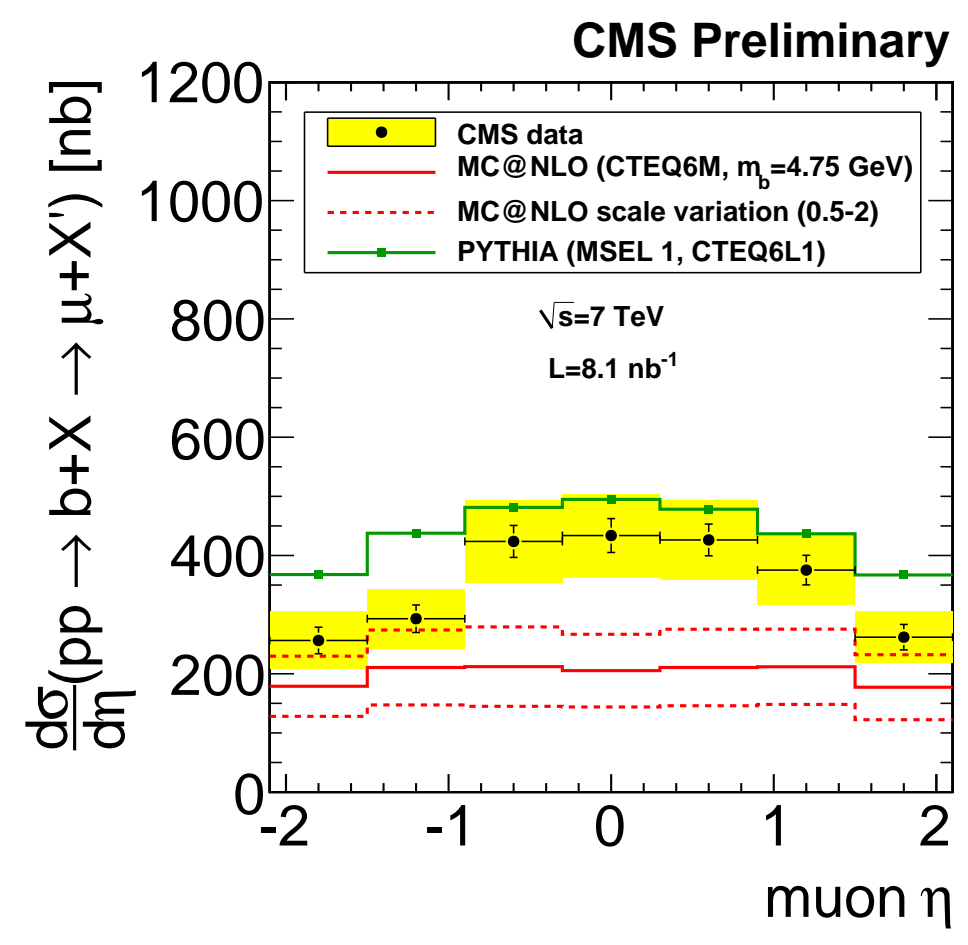

Figure 6.9: Differential $b$-quark production cross-section $d \sigma / d \eta$ for $p_{T}^{\mu}>6 \mathrm{GeV}$ as a function of the muon pseudorapidity. The black symbols show the measured cross section where the vertical error bars represent the statistical uncertainty and the horizontal error bars indicate the bin width. The yellow band shows the quadratic sum of statistical and systematic errors. The systematic error $(11 \%)$ of the luminosity measurement is not included. The dashed red lines illustrate the MC@NLO theoretical uncertainty as described in the text. The solid green line shows the PYTHIA result. 
Table 6.4: Differential $b$-quark cross-section $d \sigma / d p_{T}$ for $\left|\eta^{\mu}\right|<2.1$ in bins of muon transverse momentum. The number of $b$-events $\left(N^{b}\right)$ determined by the fit, the efficiency $(\varepsilon)$ of the online and offline event selection, and the differential cross section together with its relative statistical, systematic and luminosity uncertainty are given.

\begin{tabular}{lrrrrrr}
\hline$p_{T}^{\mu}$ & $N^{b}$ & $\varepsilon$ & $d \sigma / d p_{T}[\mathrm{nb} / \mathrm{GeV}]$ & stat & sys & lumi \\
\hline $6-7 \mathrm{GeV}$ & $2897 \pm 140$ & $0.56 \pm 0.01$ & 640 & $5 \%$ & $15 \%$ & $11 \%$ \\
$7-8 \mathrm{GeV}$ & $1479 \pm 96$ & $0.61 \pm 0.01$ & 297 & $7 \%$ & $15 \%$ & $11 \%$ \\
$8-10 \mathrm{GeV}$ & $1674 \pm 93$ & $0.67 \pm 0.01$ & 154 & $6 \%$ & $14 \%$ & $11 \%$ \\
$10-12 \mathrm{GeV}$ & $771 \pm 58$ & $0.69 \pm 0.02$ & 68 & $7 \%$ & $12 \%$ & $11 \%$ \\
$12-14 \mathrm{GeV}$ & $282 \pm 38$ & $0.76 \pm 0.02$ & 23 & $14 \%$ & $13 \%$ & $11 \%$ \\
$14-16 \mathrm{GeV}$ & $135 \pm 27$ & $0.73 \pm 0.04$ & 11 & $20 \%$ & $14 \%$ & $11 \%$ \\
$16-20 \mathrm{GeV}$ & $131 \pm 25$ & $0.78 \pm 0.04$ & 5.2 & $19 \%$ & $12 \%$ & $11 \%$ \\
$20-30 \mathrm{GeV}$ & $102 \pm 20$ & $0.77 \pm 0.04$ & 1.6 & $19 \%$ & $11 \%$ & $11 \%$ \\
\hline
\end{tabular}

Table 6.5: Differential $b$-quark cross-section $d \sigma / d \eta$ for $p_{T}^{\mu}>6 \mathrm{GeV}$ in bins of muon pseudorapidity. The number of $b$-events $\left(N^{b}\right)$ determined by the fit, the efficiency $(\varepsilon)$ of the online and offline event selection, and the differential cross section together with its relative statistical, systematic and luminosity uncertainty are given.

\begin{tabular}{lrrrrrr}
\hline$\eta^{\mu}$ & $N^{b}$ & $\varepsilon$ & $d \sigma / d \eta[\mathrm{nb}]$ & stat & sys & lumi \\
\hline$(-2.1,-1.5)$ & $773 \pm 68$ & $0.62 \pm 0.02$ & 256 & $9 \%$ & $16 \%$ & $11 \%$ \\
$(-1.5,-0.9)$ & $895 \pm 71$ & $0.63 \pm 0.02$ & 293 & $8 \%$ & $15 \%$ & $11 \%$ \\
$(-0.9,-0.3)$ & $1322 \pm 84$ & $0.64 \pm 0.02$ & 424 & $6 \%$ & $15 \%$ & $11 \%$ \\
$(-0.3,0.3)$ & $1240 \pm 82$ & $0.59 \pm 0.02$ & 434 & $7 \%$ & $14 \%$ & $11 \%$ \\
$(0.3,0.9)$ & $1333 \pm 84$ & $0.64 \pm 0.02$ & 426 & $6 \%$ & $14 \%$ & $11 \%$ \\
$(0.9,1.5)$ & $1119 \pm 75$ & $0.61 \pm 0.02$ & 375 & $7 \%$ & $14 \%$ & $11 \%$ \\
$(1.5,2.1)$ & $802 \pm 66$ & $0.63 \pm 0.02$ & 262 & $8 \%$ & $14 \%$ & $11 \%$ \\
\hline
\end{tabular}

\subsection{Systematic Uncertainties}

The systematic uncertainties of this analysis have been discussed in detail in section 4.9 and are summarized in the following. The dominant contributions are due to the description of the $u d s g$ background and of the underlying event. The modeling of $b$-quark production, semileptonic $b$-hadron decays, and the signal efficiency is better understood and has less impact on the systematic error.

The muon trigger efficiency has been determined from data in minimum bias events. The statistical uncertainty on the trigger efficiency amounts to $3-5 \%$, depending on the muon transverse momentum and pseudorapidity, and is taken as a systematic uncertainty. The muon reconstruction efficiency is known to a precision of $3 \%$. The tracking efficiency for hadrons is known with a precision of $4 \%$. This induces a systematic uncertainty of $2 \%$ on the number of events passing the event selection. The uncertainty in the tracking efficiency affects the b-fraction in the fit by about $1 \%$.

The background template consists of contributions from $c \bar{c}$ events and from light quark events, where a hadron is misidentified as a muon. The fit does not separately determine the 
$c$ and $u d s g$-content of the sample. Two effects can introduce a systematic error. (i) The $u d s g$ template determined from data could be biased. Using the PYTHIA-derived udsg template introduces a difference to the nominal fit of $1-10 \%$, depending on the muon transverse momentum and pseudorapidity bin. (ii) If the $c$-fraction of the non- $b$ background in the data were different from the value used in composing the templates, the fitted $b$-fraction would change somewhat. The error due to the background composition amounts to $3-6 \%$.

The uncertainty of the contributions of the $b \bar{b}$ production mechanisms, the modeling of the $b$-quark fragmentation and the $b$-hadron decay lead to systematic uncertainties of $2-5 \%$, $4 \%$ and $3 \%$, respectively. The systematic error due to the modeling of the underlying event is of the order of $10 \%$.

At the present early stage of the CMS experiment, the integrated luminosity recorded is known to about $11 \%$.

\subsection{Conclusions}

A preliminary result of the inclusive $b$-quark production cross-section in proton-proton collisions at a center-of-mass energy of $\sqrt{s}=7 \mathrm{TeV}$ has been determined for the first time within this thesis. The result is based on data corresponding to an integrated luminosity of $\mathcal{L}=8.1 \mathrm{nb}^{-1}$ recorded by the CMS detector during the first months of data taking in April and May, 2010.

The determination of the cross section relies on the performance of the muon and track reconstruction. The muon and TrackJet kinematical distributions were compared to the MC prediction and the simulation was found to be in good agreement with data. Furthermore, A detailed study of the dominant sources of systematic uncertainty has been performed in the scope of this work.

The measured $b$-quark production cross section was compared to LO and NLO MC predictions. The data tends to be higher than the MC@NLO prediction at low transverse momentum and central rapidity. 




\section{Part II}

\section{Construction and Commissioning of the CMS Pixel Barrel Detector}





\section{Chapter 7}

\section{The CMS Pixel Barrel Detector}

The CMS pixel detector allows for high precision tracking in the region closest to the interaction point in a particularly harsh environment characterized by a high track multiplicity and heavy irradiation. The main purpose of the pixel detector is the reconstruction of secondary vertices from heavy flavor and tau decays and the generation of track seeds for track reconstruction.

The barrel part of the CMS pixel detector was developed, designed and built at the Paul Scherrer Institute in cooperation with ETH Zürich and the University of Zürich. In this chapter, the main components of the CMS pixel barrel (BPIX) detector are introduced. An overview of the detector design and the mechanical structure is given, followed by a detailed description of the detector module and its main building blocks. In the last section, the readout and control system of the BPIX detector is explained.

\subsection{Design of the CMS Pixel Barrel Detector}

The CMS BPIX Detector [50] is composed of three cylindrical layers at mean radii of 4.4, 7.3 and $10.2 \mathrm{~cm}$ and has a length of $53 \mathrm{~cm}$. It is built from 768 highly segmented silicon sensor modules with a pixel size of $100 \times 150 \mu \mathrm{m}^{2}$ providing about 48 million readout channels (figure 7.1). The pixels are almost square shaped as emphasis has been put on achieving a similar track resolution in both the $r \phi$ and $z$ direction. The pixel detector layers are divided into two half shells built from $0.25 \mathrm{~mm}$ thin carbon fiber ladders. Each ladder is glued to an aluminum cooling pipe with $0.3 \mathrm{~mm}$ wall thickness and holds 8 sensor modules. In order to make the layers hermetic in the $r \phi$ plane, the ladders are mounted with overlap as can be seen in figure 7.1. The edge ladders in the region where two half shells meet are designed as half ladders equipped with half modules to reach full spatial coverage. Three half shells are mounted together at the endflange and form one half of the BPIX detector.

The BPIX detector is attached to four $2.2 \mathrm{~m}$ long supply tubes which carry the services along the beam pipe and house the electronics for the detector readout and control. In addition, the supply tube accommodates the cooling lines which feed the 10 cooling loops of the barrel detector mechanics. The supply tubes are a complex system in design as well as in production due to the thin radial shell thickness $(1-2 \mathrm{~cm})$, the large number of circuits, plugs and sensors, and the fine wires and thin printed circuit boards that were used. The detector and the supply tubes are connected via a six layer PCB which is mounted on the detector endflange and distributes the power and the control signals to the individual modules. The 

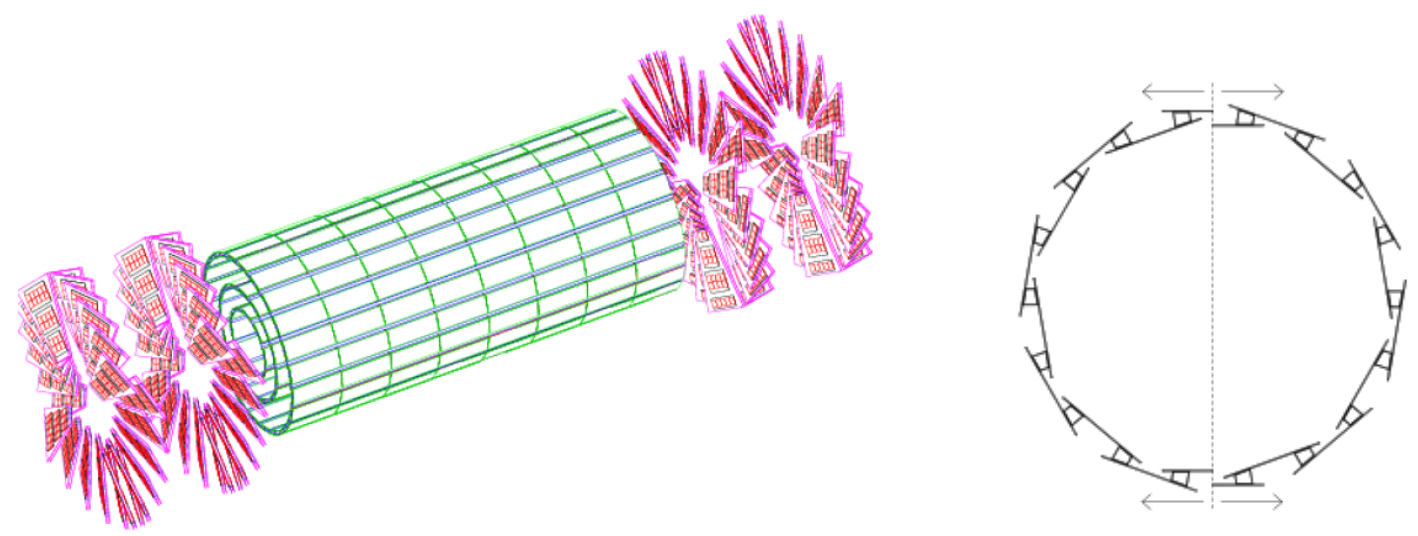

Figure 7.1: Left: Illustration of the mechanical design of the three barrel layers and the four endcap disks of the CMS pixel detector. Right: Radial cut through the mechanical frame of the first barrel layer.

final BPIX system consists of two independent half cylinders, an inner $(+x)$ and an outer $(-\mathrm{x})$, with a detector half shell and two supply tubes.

\subsection{Detector Modules}

The sensitive element of a module is a silicon sensor [100] with a dimension of $66.6 \times 18.6 \mathrm{~mm}^{2}$ and a thickness of about $285 \mu \mathrm{m}$. An array of 16 or 8 readout chips (ROCs) [101] for full and half modules, respectively, is bump-bonded to the sensor. The ROC dimension is $8 \times 8 \mathrm{~mm}^{2}$ and each ROC is segmented into 4160 pixel readout channels. On the other side of the silicon sensor a three layer high density interconnect (HDI) flex printed circuit is glued and wire bonded to the ROCs. A token bit manager chip (TBM) [102], that controls the readout of the ROCs, is mounted on top of the HDI. To fix the module to the mechanical support structure two base strips made of $250 \mu \mathrm{m}$ thick silicon nitride $\left(\mathrm{Si}_{3} \mathrm{~N}_{4}\right)$ are glued to the ROC side of the module. A power cable consisting of 6 copper coated aluminum wires is soldered to the HDI and brings analog, digital and high voltage to the module. The control and readout signals are sent through a two layer Kapton signal cable which is wire-bonded to the HDI. The HDI distributes the signals and the voltages to the ROCs.

The size of a full module is $66.6 \times 26 \mathrm{~mm}^{2}$ and the weight is up to $3.5 \mathrm{~g}$ depending on the length of the signal and power cables. The average power consumption of a full module is $2 \mathrm{~W}$. Figure 7.2 shows all components of a BPIX detector module.

\subsubsection{Sensor}

When a charged particle passes through the sensor, electron-hole pairs are produced in the sensor material which are separated by an electric field. In the CMS pixel detector the electrons are collected at the anode and passed through an indium bump to the ROC. A minimum ionizing particle crossing the sensor at an angle of $90^{\circ}$ creates an average ionization charge of about 22000 electrons. The CMS pixel detector silicon sensor adopts a double sided processed $\mathrm{n}+$ on $\mathrm{n}$ design. High dose $\mathrm{n}+$ pixels are implanted in a high resistance $\mathrm{n}$ substrate (figure 7.3). A moderated p-spray technique is used for interpixel isolation. The backside 

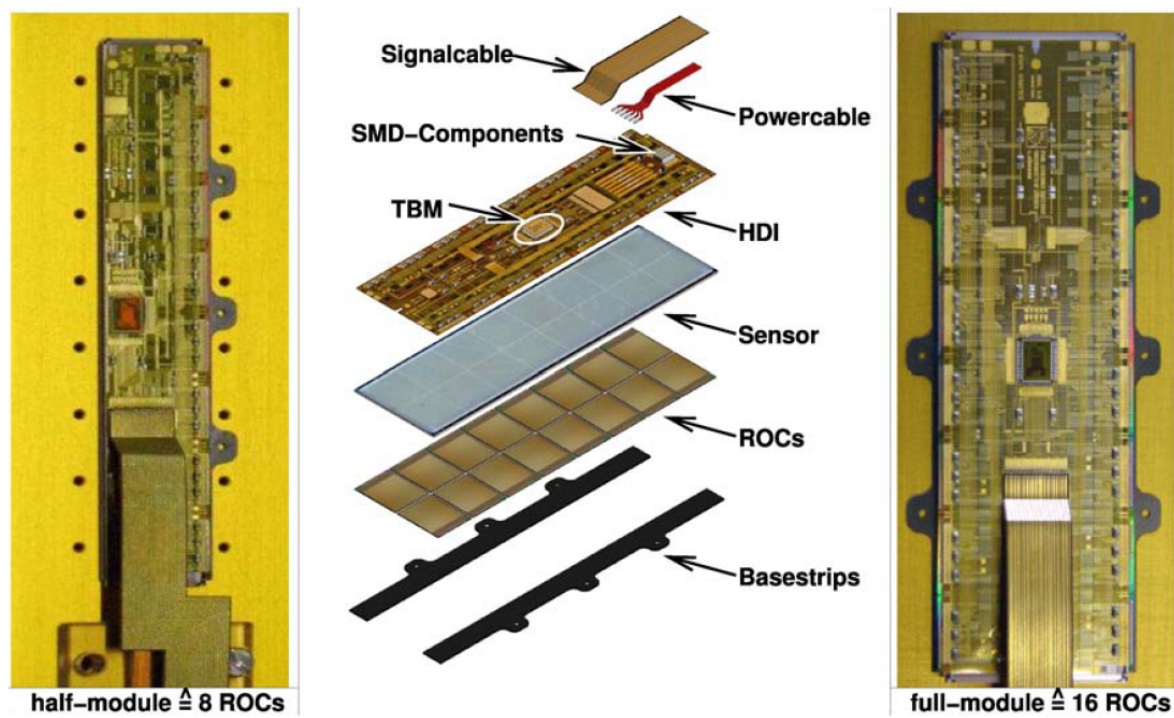

Figure 7.2: Picture of a BPIX half module (left) and full module (right). Center: The components of a pixel barrel detector module (from top to bottom): the Kapton signal cable, the power cable, the HDI, the silicon sensor, the 16 ROCs and the base strips.

is p-doped forming the pn junction. After irradiation the substrate material will be type inverted and the depletion will start at the structured n-side. Therefore a sensor design was chosen that allows partially depleted operation at very high fluence. A non-irradiated sensor can be operated at a bias voltage of $100-150 \mathrm{~V}$ while for irradiated sensors voltages up to $600 \mathrm{~V}$ have to be applied to achieve full depletion. A stable operation at these very high bias voltages is possible due to a multiple guard ring structure on the p-side that allows to keep all sensor edges on ground potential.

\subsubsection{Readout Chip}

Each silicon sensor pixel segment is electrically connected via an indium bump to a ROC pixel unit cell (PUC). The PUCs are arranged in $26 \times 80$ double columns which are controlled by the double column periphery. The double columns, the double column periphery and the chip periphery are the three main functional units of a ROC. They fulfill the task of recording the position and charge of all hit pixels with a time resolution of $25 \mathrm{~ns}$ and store the information on-chip during the L1 trigger latency. The behavior of the ROC is controlled by means of 26 digital-to-analog converter (DAC) registers which can be programmed using a modified $\mathrm{I}^{2} \mathrm{C}$ interface running at $40 \mathrm{MHz}$.

The PUC can receive a signal either through a charge deposition in the sensor or by injecting a calibration signal. Within the PUC, the signal is first passed through a two stage pre-amplifier/shaper system to a comparator where zero-suppression is applied. The comparator threshold is set by a DAC for the whole ROC but can be adjusted via a 4-bit DAC (trim bits) for each pixel individually. If a signal exceeds the comparator threshold the hit information is stored, the corresponding pixel becomes insensitive and the column periphery is notified. The column periphery writes the value of the bunch crossing counter into a time stamp buffer and issues a readout token. A column drain mechanism is initialized to read 


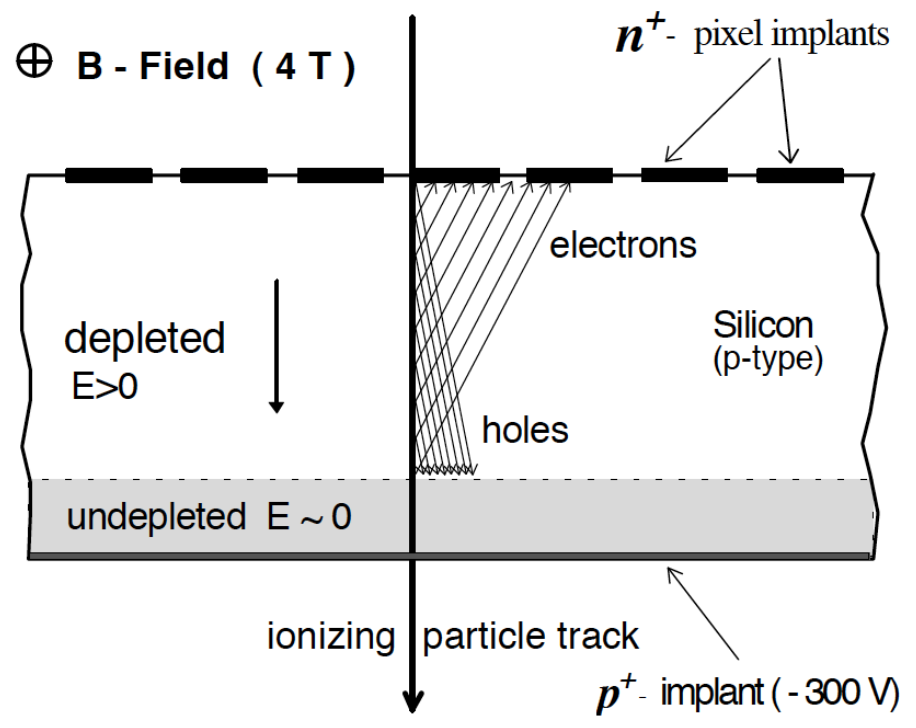

Figure 7.3: Diagram of a charged particle crossing the sensor of the BPIX module [13]. The charge introduced by the passage of the ionizing particle is collected at the high dose $\mathrm{n}+$ implants.

out the pixel hit information. Hit pixels send the registered analog pulse-height information together with the pixel address to the column periphery before being set again into data taking mode.

The double column periphery verifies the trigger by comparing the time stamp with a counter running behind the bunch crossing counter by the trigger delay. In case of agreement the column is set into readout mode and the data acquisition is stopped, otherwise the data are discarded. When the readout token arrives at the double column periphery the validated data are sent to the chip periphery and the double column is reset. The ROCs are read out serially via a $40 \mathrm{MHz}$ analog link. A picture of a BPIX readout chip is shown in figure 7.4.

\subsubsection{Token Bit Manager}

A TBM chip is wire-bonded to the HDI and controls the readout of the ROCs. Since there are two analog data links per BPIX module to the FED for the inner two layers, the TBM is configured as pairs in a dual TBM chip. The main functionality of the TBM is to synchronize the data transmission. For each incoming L1 trigger, the TBM issues a readout token. The token is passed to the ROCs and the readout is initialized. The last ROC in the chain sends the token back to the TBM. The TBM multiplexes the signal from the ROCs, adds a header and a trailer to the data stream and drives the signal through the readout link. In addition, the TBM distributes the L1 trigger and the clock to the ROCs. A schematic of the readout chain is displayed in figure 7.5 . 


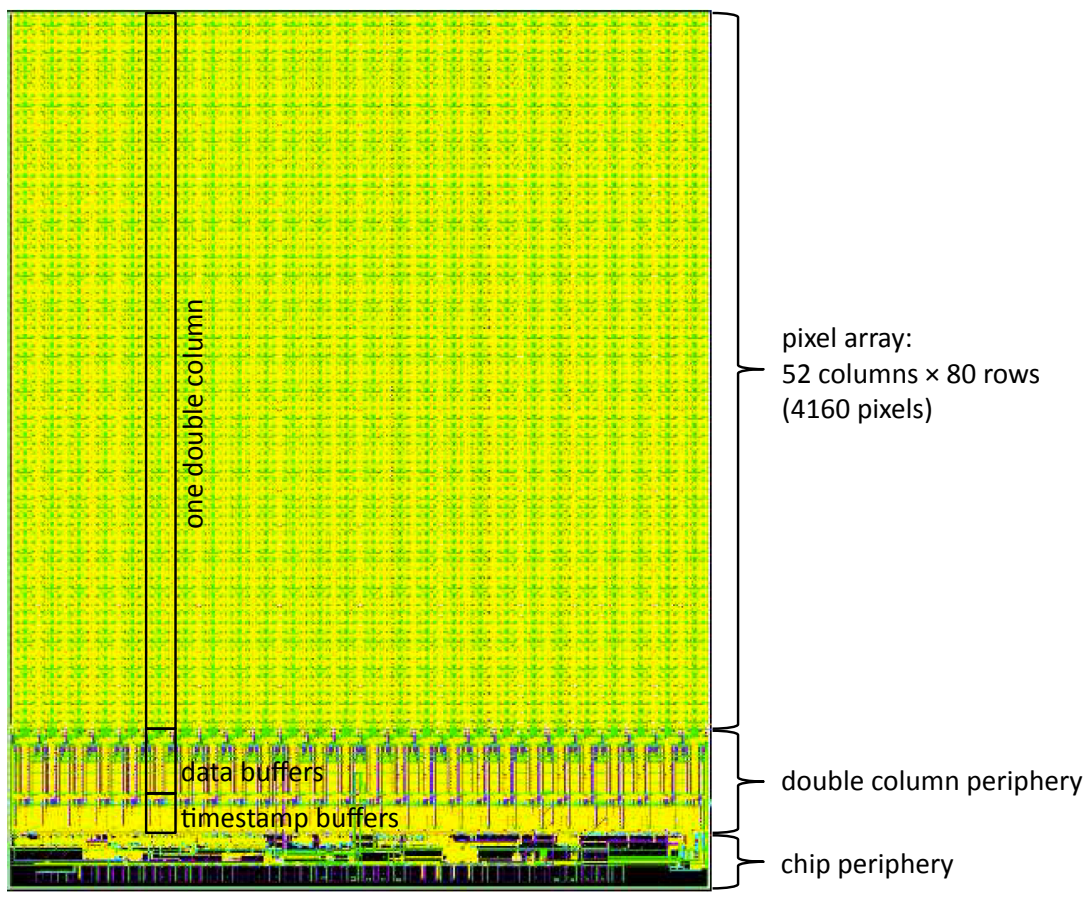

Figure 7.4: Picture of the BPIX readout chip highlighting the three main building blocks: double column, double column periphery and chip periphery [14].

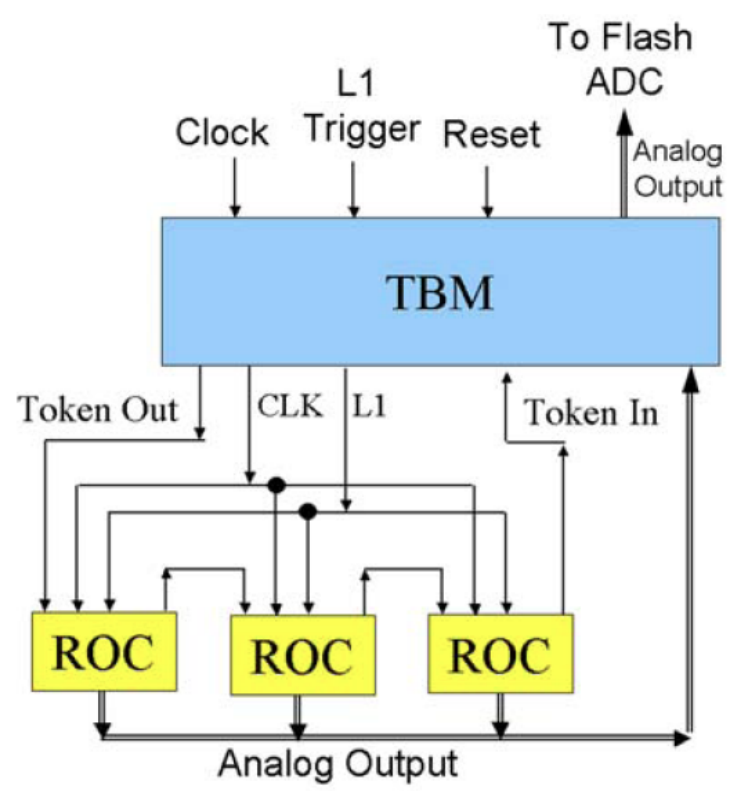

Figure 7.5: Schematics of a readout chain consisting of a TBM and a group of ROCs [102]. 


\subsection{Readout and Control System}

The BPIX readout system is organized into 64 independent readout groups consisting of analog and digital opto-hybrid circuits which serve 8, 12 or 16 modules and provide communication between the detector and the front-end modules in the underground service room. The electronics for the readout and control system are integrated on the detector supply tube. A supply tube is divided into 8 sectors which contain the power lines and the readout and control electronics of two readout groups, one serving the modules of the first two layers, the other serving the modules of the third layer.

Figure 7.6 presents an overview of the pixel readout and control system. A detailed description can be found in [103]. The system provides a very complex functionality and consists of three main parts: an analog read-out link from the BPIX modules to the frontend drivers (FED), a digital control link from the pixel front-end controller (pxFEC) to the modules and a slow control link from a standard front-end controller (FEC) to the supply tube to configure the readout electronics hosted on the supply tube. The individual components of the pixel readout and control system are described in more detail in the following.

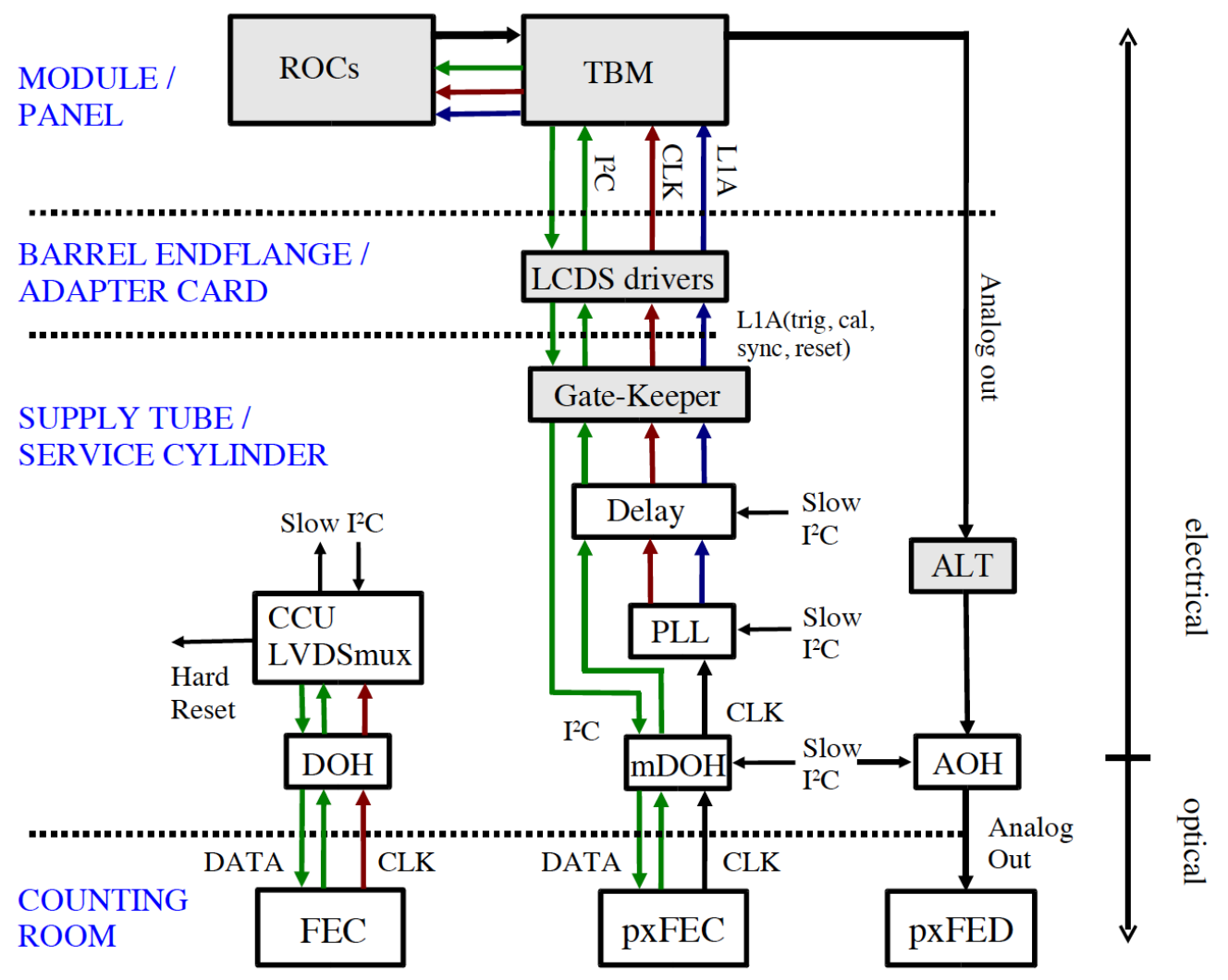

Figure 7.6: Overview of the BPIX readout and control system. 


\subsubsection{Analog Chain}

An example of an analog readout signal of a module with a single pixel hit is shown in figure 7.7. The data stream contains the readout signals from each ROC in the readout group preceded by the TBM header and ended by the TBM trailer. The TBM header uses eight clock cycles and starts with three very low analog levels called ultra-black (UB) and a black level which defines the zero level of the differential analog signal. The additional four levels contain the event number information. The readout sequence of a ROC begins with a UB level, a black level and a level called last DAC which comprises the value of the DAC addressed by the last programming command. The hit information is transmitted in 6 clock cycles encoding the pixel address and the pulse height information. The pixel address consists of the pixel row and column number coded in six discrete analog levels. The TBM trailer consists of two UB levels and two black levels followed by four clock cycles transmitting the TBM error status.

The data stream which contains all hit information belonging to a single trigger is sent out by the TBM through the module Kapton cable. The Kapton cable consists of differential analog lines separated by quiet lines from the lines for the fast digital signals. The analog signals are split from the digital signals on the endring PCB. A single Kapton cable brings the analog signals of one readout group to the printed circuit board on which the Analog Optical Hybrids (AOHs) [104] are placed. The electric analog signals are amplified in an Analog Level Translator (ALT) chip and converted into $40 \mathrm{MHz}$ analog optical signals in the AOHs. Each $\mathrm{AOH}$ is equipped with 6 lasers which drive the signal through optical fibers to the front end drivers.

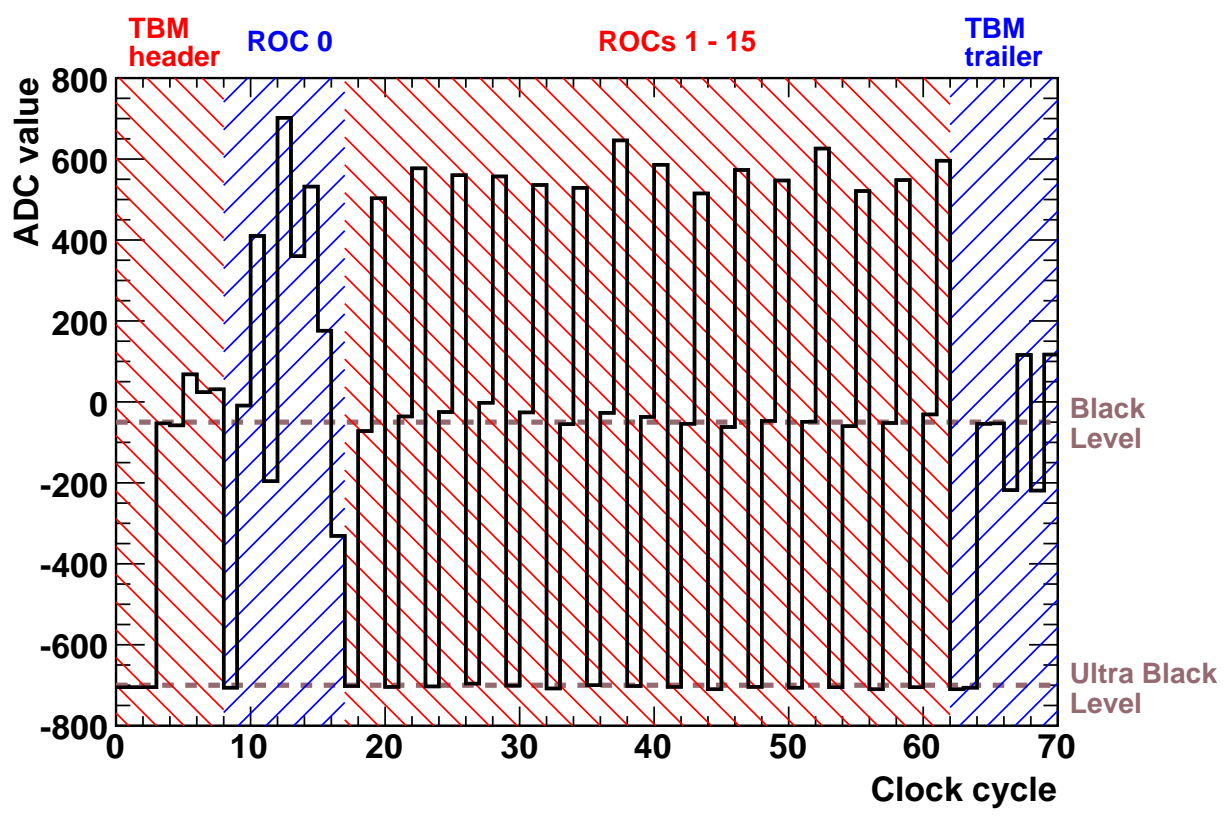

Figure 7.7: Analog readout of a full module with one hit in ROC 0. 


\subsubsection{Front End Driver}

A total of 32 front end drivers (FEDs) [106] are setup in two 9U VME crates located in the CMS underground service room. The optical fibers connecting the AOHs to the FEDs have to cover a distance of about $60 \mathrm{~m}$. A FED has 36 optical inputs each equipped with an optical receiver and an ADC. The FED receives the analog data, digitizes the signals at the LHC frequency and decodes the pixel address information. It then builds event fragments and sends them to the central DAQ system. Alternatively the FED can be operated in a transparent mode making unprocessed ADC output data available for calibration and testing purpose. A programmable offset voltage can be set for each optical input in order to compensate for bias shifts in the analog signal.

\subsubsection{Supply Tube}

Four supply tube half cylinders hold the readout and control circuits of the pixel detector. Each sector of the supply tube includes an analog opto-board with 6 AOHs and a digital optoboard with two digital opto-hybrids (DOHs) [107] on the detector near side. The front-end controller modules send the clock and trigger information together with other control signals through the DOHs to the detector. A DOH is connected to four optical fibers, two for receiving and two for sending signals. The LHC clock and trigger information is encoded in one signal which is sent over a single fiber to the DOH. A phase locked loop (PLL) chip [108] is used to split the clock from the trigger information before sending it to the detector modules. The relative phases of all control signals are adjusted using a DELAY25 chip [109]. A Gatekeeper chip converts the low voltage differential signal (LVDS) used by the PLL and the DELAY25 chip to a low current differential signal (LCDS) used by the pixel front-end chips. The electronic devices needed in the digital control circuit are mounted on the digital opto-boards. In each sector 44 optical fibers are needed for communication with the front-end modules, 36 for the analog readout and 8 for the digital control.

A communication and control unit (CCU) board is placed in the central sector of the supply tube. It is the core component developed for slow control, monitoring and timing distribution and is described in more detail in the next section. The slow control signals like temperatures, pressures and humidities are also brought together in the central slot and connected by dedicated slow control adapter boards to the readout cables. A schematic view of a supply tube half cylinder is shown in figure 7.8 .

\subsubsection{Communication and Control Unit}

The pixel detector front end control system consists of four CCU boards equipped with 9 CCU chips [110]. Each board supervises one quarter of the detector. The slow control links are implemented as a ring architecture. A ring consists of 9 CCUs, two optical drivers and receivers that bring clock, trigger and control data to the CCUs and a front-end controller (FEC) [111] which is the master of the network. The CCUs distribute the digital control signals to the individual readout boards in each sector. A CCU chip supports two $\mathrm{I}^{2} \mathrm{C}$ channels to communicate with the front-end readout electronics, and three PIA channels to generate the necessary signals to reset the AOHs, DOHs and ROCs of one sector. Eight CCUs are used for the control of the eight sectors, the ninth CCU is a dummy CCU used for redundancy.

Since a sizable number of front-end channels depend on the same control link, a very high reliability of the system is of utmost importance. A CCU failure leads to a loss of communi- 


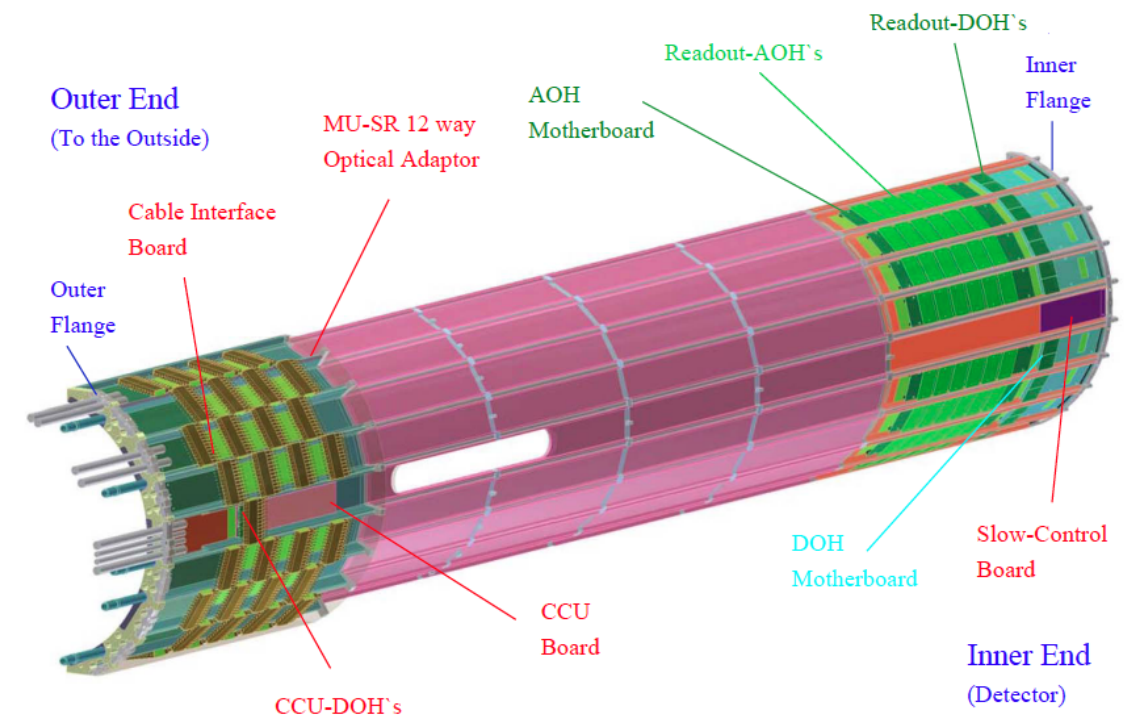

Figure 7.8: Schematic view of one of the four BPIX supply tubes.

cation to all electronics attached to the CCU. A redundancy scheme based on doubling signal paths and bypassing of interconnection lines, between the CCUs and between the CCUs and the FEC, is supported. The dummy CCU allows to mitigate a single DOH failure. The $\mathrm{CCU}$ is equipped with two DOHs which form separated control rings and thus ensure a high operational reliability. The DOHs on the CCU board are programmed by the first two CCU chips. The layout of the CCU board is shown in figure 7.9.

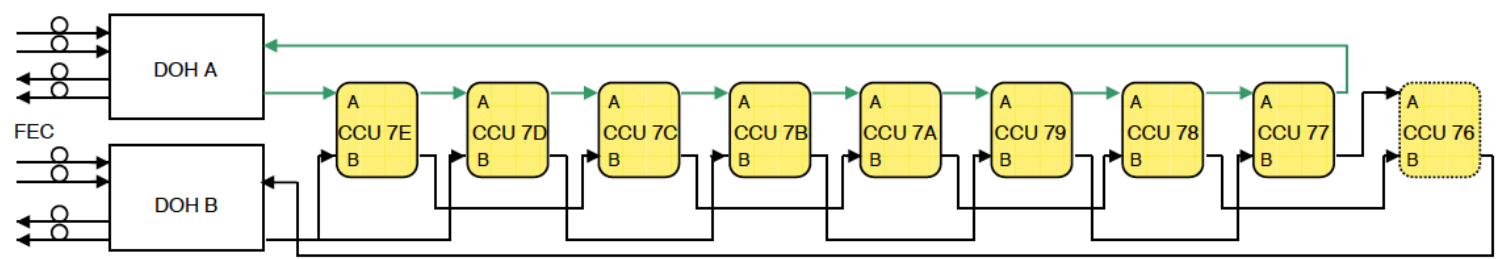

Figure 7.9: Layout of the BPIX CCU board showing the CCU chips, the DOHs and the doubled interconnection lines. The doubled signal paths (called A and B) and the ability of bypassing of interconnection lines ensures the high operational stability of the system.

\subsubsection{Front End Controller}

The pixel detector uses two different types of front-end controllers: a Pixel FEC (pxFEC) and a standard Tracker FEC (trFEC). In both cases, 8 mezzanine daughter cards are mounted on the FEC mother cards. The pxFEC is used to program the detector front-end chips (TBMs, ROCs) and to distribute the clock and trigger signal to the modules. The trFEC provides 
communication with the CCUs and the programming of the front-end devices (AOHs, DOHs, PLL, DELAY25). While the trFEC uses the standard $\mathrm{I}^{2} \mathrm{C}$ protocol, a pixel specific modified $\mathrm{I}^{2} \mathrm{C}$ protocol is implemented in the pxFEC.

The FEC performs a transmission verification by comparing the number of bytes sent to the number of bytes returned by the front-end and checking the returned hub/port address. Status bits with the result of the comparison are set by the FEC and stored for possible review. The transmission verification by the FEC is not used in standard operation mode. 


\section{Chapter 8}

\section{Construction and Commissioning of the CMS Pixel Barrel Detector}

The CMS BPIX detector system was assembled and fully tested at PSI before it was transported to CERN [112]. A slice of the CMS control and data acquisition system has been setup at PSI in order to have a tool for operating and testing larger segments of the pixel detector.

A main focus of the hardware related work during this thesis was on the setup of the test system at PSI and the development of software algorithms needed for testing, calibrating and monitoring all detector components during the integration at PSI and later during the installation phase at CERN. In addition, this work contributed to the successful completion of the construction, commissioning and installation of the final detector system.

In the first two sections the development of the testing procedure is described, before the construction of the BPIX detector is addressed. In particular the technique for mounting the modules on the support structure, the assembly of the detector control and readout electronics on the supply tube and the integration of the final system are explained. The following section focusses on the installation of the BPIX detector into the CMS detector. In the last section the results of the system tests are discussed and the performance of the BPIX during the first running period is reviewed. The chapter concludes with a summary.

\subsection{Low Level Hardware Testing Procedure}

The goal of this work was to develop a reliable and fast testing procedure in view of the commissioning of the BPIX detector at PSI and the installation of the detector inside CMS. Due to the tight schedule of the final integration of CMS, a well established procedure which allows to determine in a very short period of time whether the central elements of the detector were working was of utmost importance during the installation. Furthermore emphasis was placed on the realization of a standalone testing procedure which would not depend on the availability of the standard CMS software framework. The implementation of the standalone software allows direct communication with the main hardware components and is thus very well suited to quickly identify potential failures. 


\subsubsection{Test Setup}

The BPIX control and data acquisition system set up at PSI was built from front-end modules equivalent to the ones which will be used during data-taking at CERN. Initially, a group of 12 detector modules were operated (System12). The modules were connected through an endring print to an $\mathrm{AOH}$ board equipped with $2 \mathrm{AOHs}$ which transmit the analog optical signals to the FED. A pxFEC, a trFEC and a fully equipped DOH board formed the digital detector control. The System12 setup was mainly used to test the individual components of the readout system and to gain operational experience with the very complex system. Furthermore, it presented a useful tool for testing and debugging new software developments.

A commissioning system with the true mechanical structure and prototype supply tubes was assembled before the construction of the final detector. In the commissioning system only two sectors were equipped with modules. A second test stand was established which allowed to operate the commissioning system independent of System12. This test stand was later used to commission the two halves of the final detector system. The final detector system was tested sector-wise since only one sector could be connected to the readout at a time due to the limited number of optical fibers and FED modules available.

\subsubsection{Software Architecture}

The BPIX readout and control system consists of three main parts (see section 7.3): the analog readout link between the modules and the FED, the digital control link between the modules and the pxFEC and the slow control link between the CCUs and the trFEC. At P5 the FED and FEC boards are installed in different VME crates in the CMS underground service room. Since one crate of electronic boards is controlled by one PC, the software used for communication is implemented as a distributed system.

The standalone BPIX online software has been developed using the socket technology [113] provided by the framework of Python [114]. The supervisor processes - called trFECProcess, pxFECProcess and FEDProcess - are implemented in $\mathrm{C}++$ and communicate with the trFEC, pxFEC and FED VME boards, respectively. Similarly, the PSProcess controls the CAEN power supply. The clock and trigger information is sent from the TTC board which is programmed using a standard CMS XDAQ [115] process.

Through the Python socket interface the user can send messages to the control processes and receive the answers (one query at a time). In figure 8.1, a schematic overview of the different software components is shown. In a standard testing sequence, first the clock and trigger distribution is started and the CAEN power supply is turned on. Then the delay settings are adjusted by issuing commands in the trFECProcess, the BPIX modules TBMs and ROCs are programmed and the readout is initialized. In the end, the analog data is read out via the FEDProcess and the digital readback is verified using the pxFECProcess.

For the offline data analysis the ROOT [80] framework was used. In addition, a ROOT program called FEDScope operated as a digital oscilloscope to monitor the FED output.

\subsubsection{Testing Sequence}

The test procedure is implemented as a bottom-up approach and is divided into four stages which are discussed in the following. 


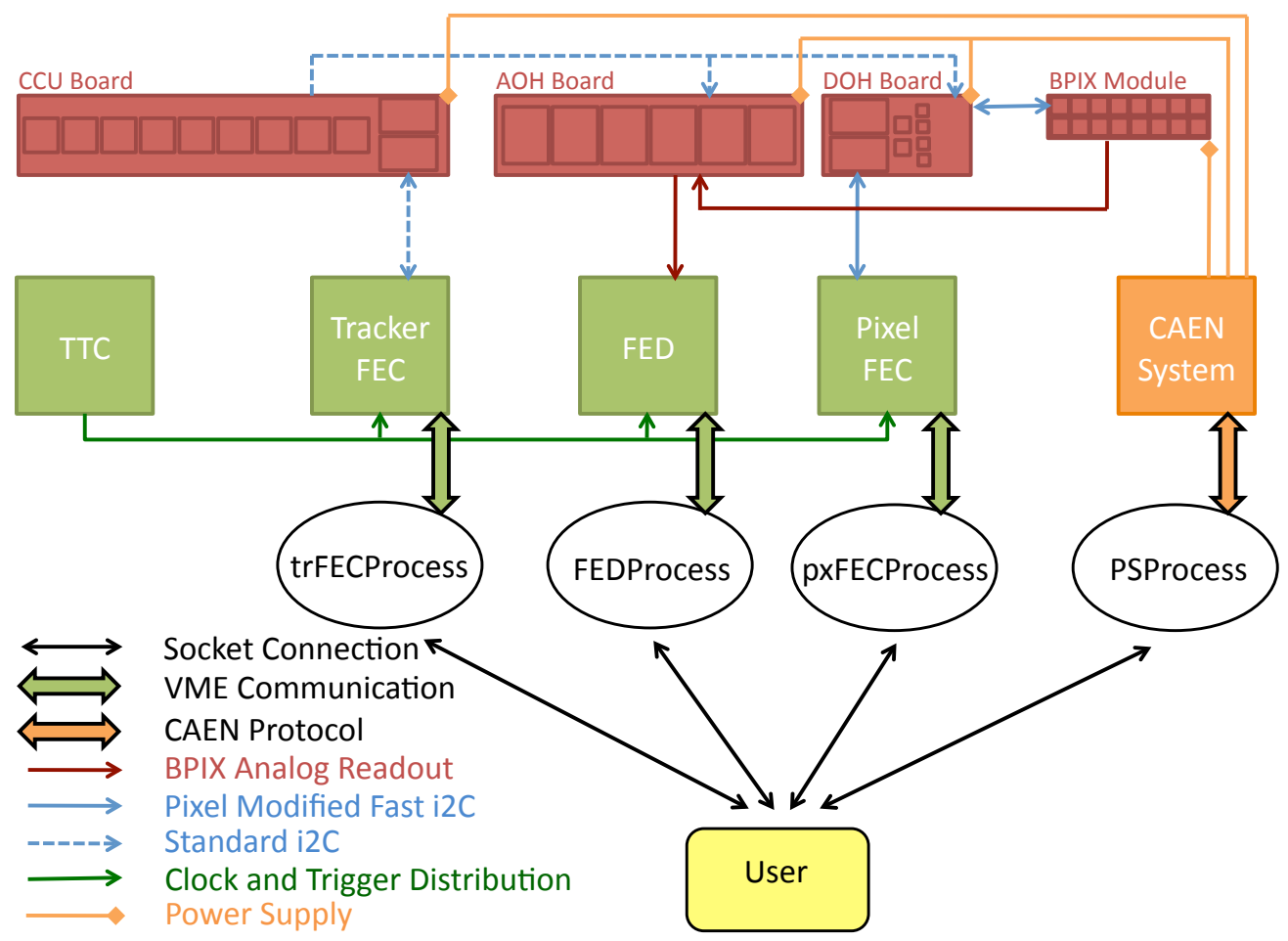

Figure 8.1: Schematic view of the BPIX low level hardware test software architecture.

1. Functionality of the detector control and communication system

First, the proper working of the CCU ring architecture, the redundancy mechanism and the correct programming of the $\mathrm{I}^{2} \mathrm{C}$ devices is verified. In addition, the hard resets issued by the trFEC and sent through the CCU reset line to the AOHs, the DOHs and the ROCs are tested. The $\mathrm{AOH}$ and $\mathrm{DOH}$ resets can be checked by reading the values in the chip registers. Since at this point of the testing procedure communication with the detector modules is not yet established, an alternative method has to be used to spot the effect of the ROC reset. This can be done by monitoring the power consumption via the PSProcess as the digital current is increased by a hard reset.

\section{Performance of the analog readout chain}

If the tests of the detector and the control and communication system prove successful, the test sequence is continued, from now on investigating one readout group at a time. In a first step the mapping between the $\mathrm{AOH}$ and the FED channels is checked. Each $\mathrm{AOH}$ is equipped with 6 lasers for which the bias and the gain can be adjusted individually. The optical fibers are combined in groups of 12 which then connect to one FED input. A FED has three inputs and serves the two readout groups of one sector. The mapping is verified by increasing the bias of a given laser and checking the light intensity received in the corresponding FED chan- 
nel. In case a wrong match is found the problem is resolved by either redoing the connection on the supply tube or updating the original map. In a next step, the noise and the gain on the analog transmission line is measured by performing a scan of the full laser bias range. As an example the light intensity, the slope and the noise as a function of the laser bias are shown for one $\mathrm{AOH}$ channel in figure 8.2. Poor or dirty connections strongly affect the light transmission since light might be either absorbed (which lowers the gain) or scattered back to the laser (which increases the noise). Channels with a noise value of more than 4 ADC counts or very low gain could in most cases be improved by re-cleaning the optical connections.

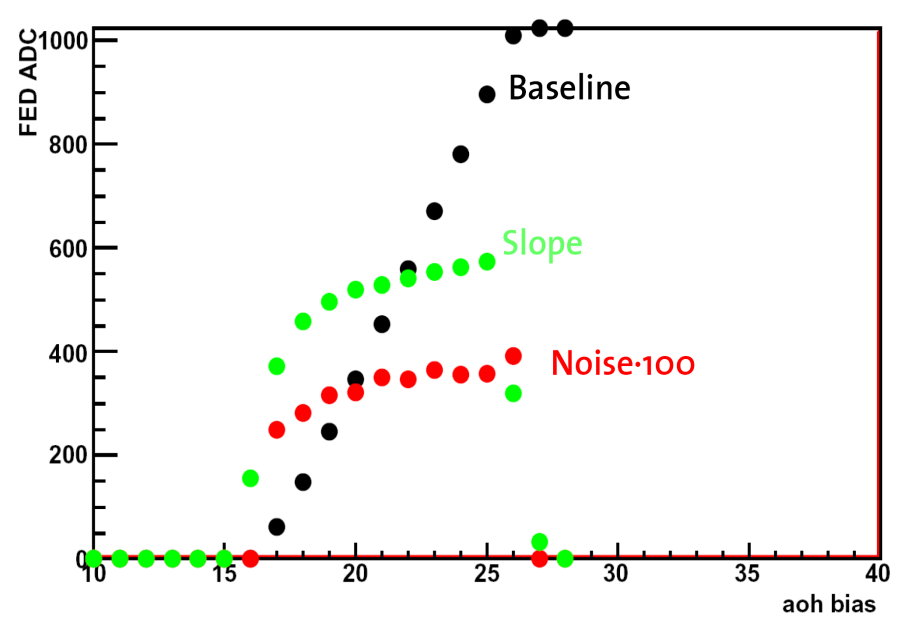

Figure 8.2: Baseline, slope and noise of an analog optical fiber as a function of the bias of the laser measured at the FED.

\section{Performance of the digital readback mechanism}

In order to establish $\mathrm{I}^{2} \mathrm{C}$ communication with the BPIX modules the delays in the digital readout circuit have to be adjusted. The phase of each digital transmission line can be independently programmed in steps of $0.5 \mathrm{~ns}$ from 0 to $25 \mathrm{~ns}$ using the corresponding register in the Delay25 chip. Additionally, the PLL chip implements a function that generates 12 different clock phases evenly distributed between 0 and $12 \mathrm{~ns}$.

In CMS the LHC clock and the L1 trigger decision are transmitted from the counting room to the detector using one single fiber. To achieve this, both the clock and trigger signals are encoded as a single signal as schematically illustrated in figure 8.3. At the receiving end the signal is decoded by the PLL chip and sent via two separate lines, LHC clock (CLK) and Calibrate/Trigger/Reset (CTR), through the Delay25 chip to the BPIX modules. In addition, the CLK signal is split in the Gatekeeper chip and one line (RCK) is returned and sent again through the Delay 25 chip. The digital programming and control data (SDA) also goes through the Delay25 chip. If the gate is open the SDA is transmitted to the BPIX modules which sends the acknowledge signal (RDA) back, otherwise the data packet is returned in the gatekeeper. The digital circuit is illustrated in figure 8.4.

Since the Delay25 and the PLL chip serve all modules of the corresponding readout group, they cannot be used to compensate for delays between modules caused by different lengths of their signal cables. For this purpose further delays can be added on the endring PCB. 


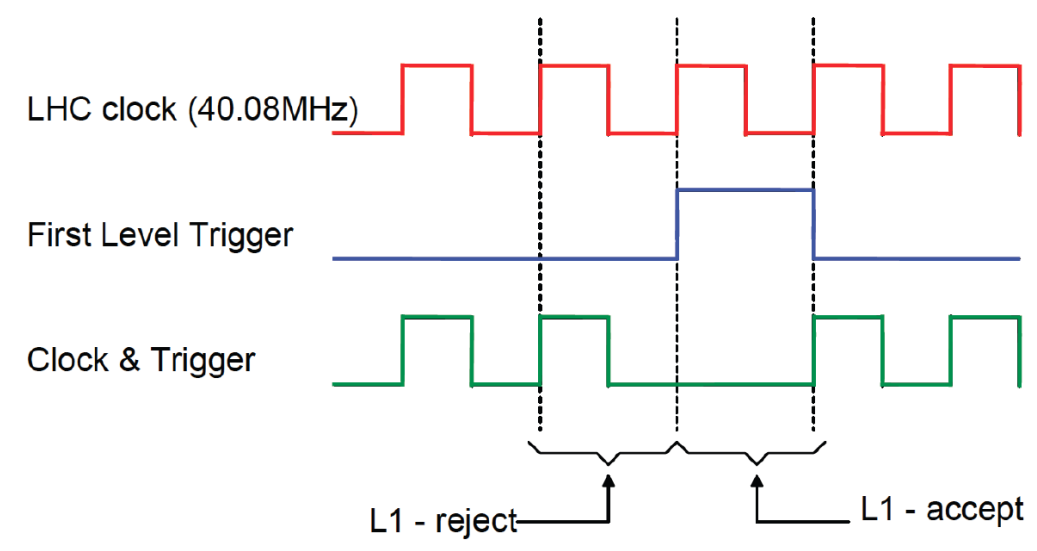

Figure 8.3: Combined coding of the LHC clock and L1 trigger signals [108]. The encoding is done such that the coded signal is identical to the clock signal if the L1 trigger issues a reject decision. If however an event is accepted by the L1 trigger, the coded signal stays at the low level for the duration of one clock cycle.

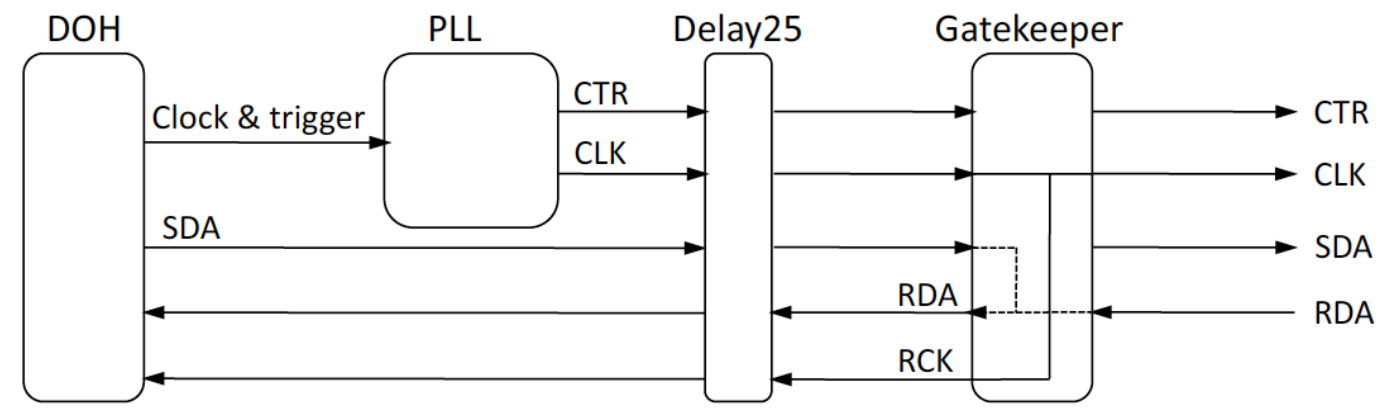

Figure 8.4: Illustration of the BPIX digital circuit.

The SDA signal can only be decoded by the TBM if it is in phase with the CLK signal, so that the start and stop conditions are recognized correctly. The working region for sending data is common to all modules in a readout group and is determined by performing a scan of the Delay25 SDA register whilst the delay of the CLK signal is kept at a constant value. In each point of the scan, the value of the DAC register that regulates the analog voltage used by the ROC is increased for all ROCs and the analog current is watched via the PSProcess. If the current increases, the programming is successful and a valid delay setting is found. It should be mentioned that this method is time consuming as the current reading on the power supply is stable only after about $3 \mathrm{~s}$. The plateau of the correct phase is about $6-8 \mathrm{~ns}$ and a working point in the middle of the plateau is chosen.

\section{TBM and ROC programming and module analog readout}

After an operation point for module programming is found, the digital readback of the modules is tested. If a programming command is received by a module, the TBM sends an acknowledge sequence which includes the TBM address. The TBM addresses take values between 0 and 
31 and are assigned according to a predefined address scheme within a readout group. The address assignment is checked by going through a scan of the RCK delay: programming commands are sent to the modules and the returned addresses are decoded in the pxFEC. An example of a RCK scan is shown in figure 8.5.

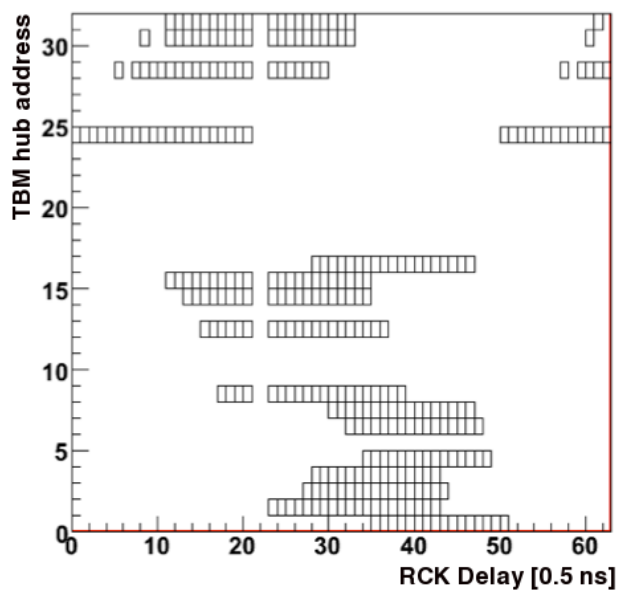

Figure 8.5: Digital readback of 16 modules in a readout group as a function of the RCK delay. The boxes indicated the RCK values at which the programming commands were received and returned correctly by the TBM. The plateau of correct phase is about $10 \mathrm{~ns}$. It is shifted among the modules in a readout group since the signal path depends on the position of the module on the detector mechanics.

The RCK delay range in which the acknowledge of a module is received has a length of about $10 \mathrm{~ns}$ and depends on the module position on the detector mechanics since the phase shift between RCK and RDA signal is due to different cable lengths and the delay added on the endring print. The RCK delay however is set globally for the whole readout group. In order to determine a working point common to most modules in a readout group, a two dimensional scan of RCK delay versus RDA delay is performed. The setting of the RCK and RDA delay does not influence the data taking as the digital readback is only a diagnostic tool. It is chosen such that the readback of a maximum number of modules is received and it is re-adjusted if needed.

Almost all readout groups showed satisfactory results when testing the readback. The most common failures were caused by bad connections either of the module cables at the PCB or between the PCB and the supply tube and could be recovered.

Besides the information about the relative displacement of the RCK delay setting range between the modules in a readout group was used to measure the signal speed in the Kapton signal cable. Since the length of the signal cables and their position on the endring print were known, the signal speed could be calculated. The measured value of $7 \pm 2 \mathrm{~ns} / \mathrm{m}$ is in good agreement with the technical specification of $5.39 \mathrm{~ns} / \mathrm{m}$.

In the last stage of the low level hardware tests the functionality of the modules is verified. The trigger signal is sent to the modules to initialize the readout and the analog signal is examined using the FEDScope. First, the mapping between module addresses and FED channels is reviewed. The readout speed of the TBM can be programmed to either $20 \mathrm{MHz}$ 
or $40 \mathrm{MHz}$. Changing the readout frequency of the TBM is well visible on the FEDScope and thus can be used to identify the FED channels corresponding to a given TBM address. Furthermore, the presence of a correctly digitized analog signal in the FED confirms the functioning of the trigger distribution, the token mechanism and the module readout sequence.

The procedure turned out to be well suited for a fast identification of broken detector modules since the malfunctioning of modules was in most cases due to wire-bonds which have been damaged during the handling. The corresponding failure modes, for instance the prevention of the token passage or the trigger distribution, could be spotted quickly when performing the testing sequence described above.

\subsection{Performance Tests and Calibrations}

More detailed performance tests and calibrations are performed using the CMS pixel online software implemented in the XDAQ framework. The following sequence of test and calibration stages is processed [116]:

1. Repeat the adjustment of the delay settings within the digital communication circuit.

2. Adjust the sampling point (delay and phase setting) in the FED for the digitization of the analog pulse.

3. Adjust the laser bias in the AOHs. The optimal setting is found by increasing the bias current until the separation between the ultra-black and the black level saturates. The $\mathrm{AOH}$ bias calibration is temperature dependent and has to be redone each time the operating temperature of the detector changes.

4. Set the ultra-black signals of the ROCs and TBMs to the correct amplitude [101]. The TBM and ROC ultra-black levels have to be readjusted in case the previous item changes.

5. Tune the offset in the FED to set the black level of the arriving analog signal to a predefined value in the middle of the ADC range. Due to the strong temperature dependence of the AOHs, the position of the black level baseline within the FED ADC range has to be monitored constantly and re-adjusted if the drift is too high.

6. Select a threshold and delay setting for each ROC in such a way that the injected test charge is correctly registered and readout. The amplitude of the injected signal is set by programming the corresponding DAC register $\left(V_{c a l}\right)$.

7. Perform the address level calibration. As discussed earlier, the pixel row and column address is encoded in 6 discrete analog levels which have to be well separated for being correctly decoded by the FED. The position of the six address levels is determined by measuring the levels of all pixels in a ROC and overlaying them in a histogram (figure 8.6). The decoding limits placed in the center between to neighboring peaks are then downloaded to the FED.

8. Run the pixel alive test. In this test, charges above threshold are injected into all pixels in a ROC and the correct response of each pixel is verified.

9. Check the high voltage connection of each module. 


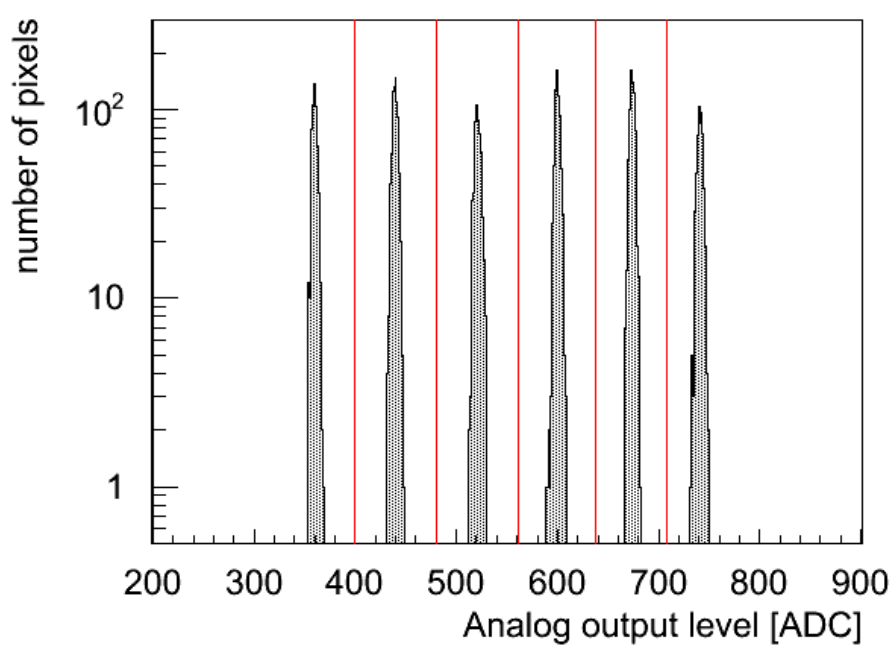

Figure 8.6: Address-levels of all pixels in a ROC as received by the FED. The red lines are the separation limits used for the decoding of the pixel addresses in the FED.

10. Determine the threshold and the noise of each pixel. This is done by measuring the so-called S-Curve which is the pixel response efficiency as a function of the injected charge. An S-Curve measurement recorded during module testing at PSI is shown in figure 8.7. The charge value corresponding to a response efficiency of $50 \%$ defines the threshold, while the noise is proportional to the width of the region where the efficiency changes from $0 \%$ to $100 \%$. The S-Curve is measured for a subset of 81 pixels per ROC which was found to be sufficient to determine the average noise and threshold per ROC. The amplitude of the injected signal has been calibrated during the module tests using test data from X-ray sources of known energies [105]. An average slope of 65.5 electrons per DAC unit and an offset of -114 electrons was determined, the values change by up to $15 \%$ from ROC to ROC.

11. Calibrate the analog pulse height. An exact calibration of the pixel charge measurement is crucial for a precise position resolution since the hit position is interpolated from the charge information of all pixels in a cluster. The calibration is performed by injecting signals with increasing amplitudes to each pixel and measuring the analog pulse height. An example of a measurement of one pixel is shown in figure 8.8. For each pixel about 30 charge values are injected. The pulse height curve is approximately linear below saturation at about 45000 electrons and can be parametrized by the slope (gain) and offset (pedestal) of a linear fit.

The result of these tests and calibrations at the different stages of the BPIX detector construction will be reviewed in the following. 


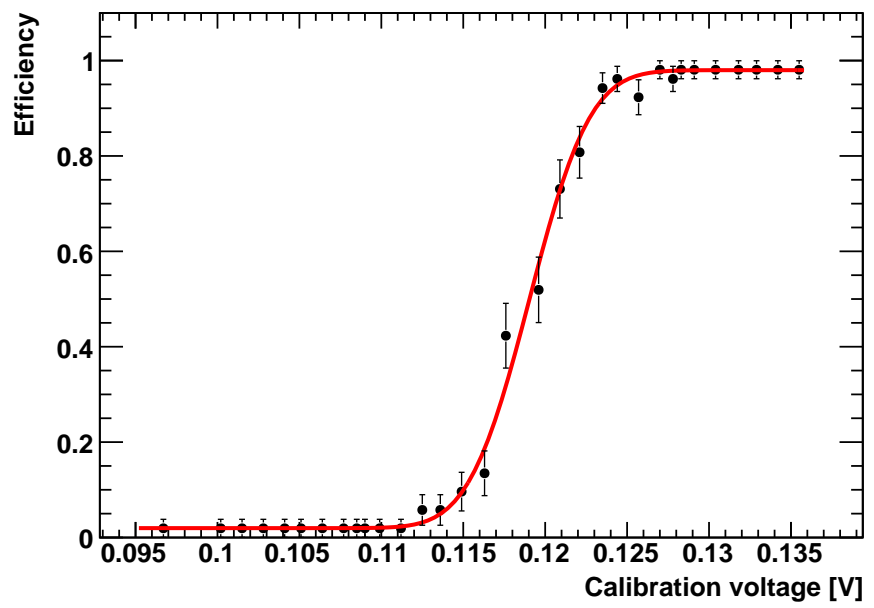

Figure 8.7: Measurement of the pixel response efficiency as a function of the injected charge fitted with an error function (S-Curve). The value corresponding to a response efficiency of $50 \%$ determines the threshold, the noise is proportional to the width of the error function.

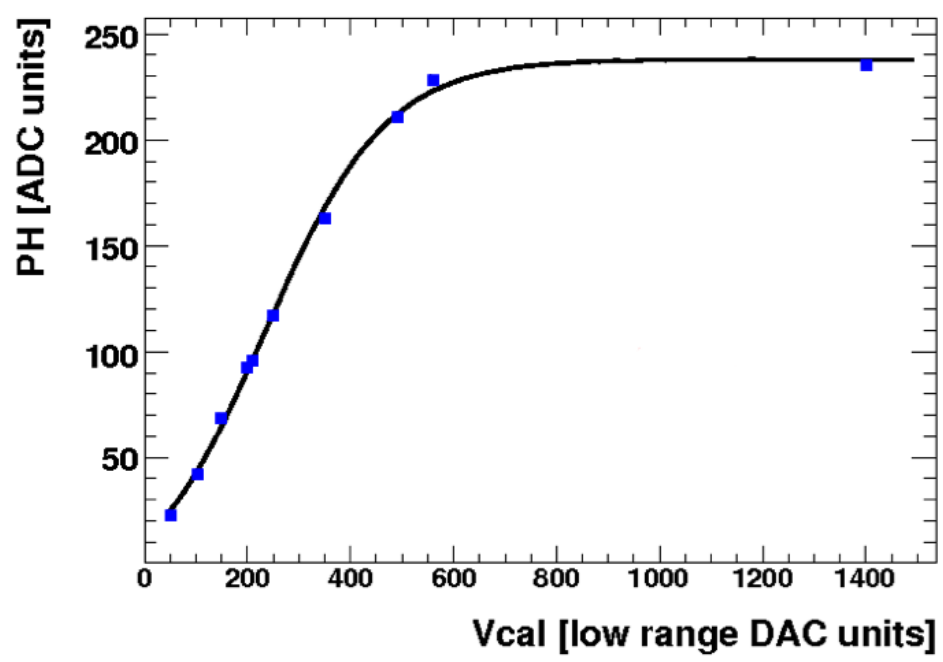

Figure 8.8: Measured pulse height as a function of the amplitude of the injected signal. One $V_{\text {cal }}$ DAC unit corresponds to 65.5 electrons. 


\subsection{Construction}

The integration of the CMS BPIX detector took place between December 2007 and May 2008. The modules were mounted on the support structure at PSI while the assembly of the supply tubes was done at the University of Zürich. The final system was assembled and fully commissioned at PSI. Thereafter, the system was transported to CERN in a fully functional state, without disconnecting any components.

\subsubsection{Module Mounting}

The BPIX modules were built [117] and extensively tested at PSI [105], [118], [119]. The goal of the module tests at PSI was to verify that all pixels function correctly, each ROC can be programmed properly, and all calibrations of a module produce meaningful results. All modules were graded and only the best quality modules were used in the final system. In total, 948 modules have been manufactured and tested. The carbon BPIX detector mechanics was fabricated at the University of Zürich.

We mounted the 768 modules of the BPIX detector manually on the carbon support structure. The three layers of the support structure are divided into half cylinders and consist of 10, 16 and 22 ladders, respectively. The ladders are arranged alternating on the inner and the outer surface of the half cylinders and accommodate eight modules each. The first four modules were connected to the endring prints on the $-z$ side of the detector, the remaining four modules to the prints on the $+z$ side. The signal and power cable of the module had to be adjusted to match a particular module position on the mechanical structure. An elaborate procedure has been established to prepare the modules before mounting:

- Setting the TBM address by removing wire bonds according to a predefined address scheme.

- Cutting the Kapton signal cable to a precision of $0.5 \mathrm{~mm}$ compared to the predetermined length.

- Bending the signal cable with the help of a special bending tool that ensures a well defined bending radius and an exact position of the bending.

- Cutting the power cable with a precision of $\approx 2 \mathrm{~mm}$.

- Soldering a plug to the shortened power cable.

- Attaching the power cable to the signal cable.

In order to protect the sensitive structures of the modules from mechanical stress and touches, the body of the module was stored in a box during all manipulations of the cables. Afterwards, the module was uncased and put on a jig equipped with two small pins that fit through the screw holes in the module base plate to keep the module in place. A dedicated mounting tool had been designed to facilitate the demanding task of lifting the module from the jig and placing it onto the ladder. The mounting tool is a clamp in which the module position is fixed with the aid of a $100 \mu \mathrm{m}$ thin stainless steel sheet taking hold of the module base plate. It has a mechanical guidance to place the module on the ladder and two feedthroughs for the screwdriver. This allowed to screw the modules in a protected way onto 
the ladders. The module cables were fixed at the end of the ladder with a tiny cable clamp and connected to the PCB placed on the detector endflange. The mounting procedure is illustrated in figure 8.9.
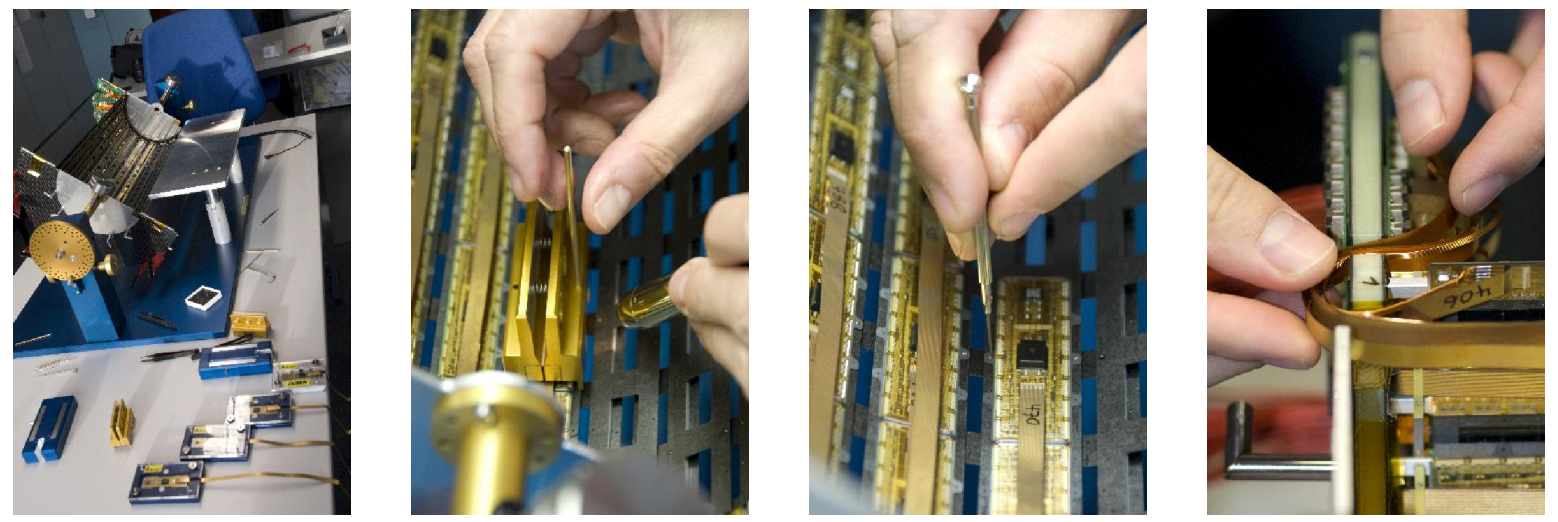

Figure 8.9: Illustration of module mounting procedure: 1. Working place for the detector assembly. 2. Placing the module on the shell. 3. Screwing the module to the shell. 4. Placing cables at the end-flange.

The basic functionality of the modules was verified at different stages of the mounting procedure. This included a measurement of low and high voltage, a scanning of the module TBM addresses and a measurement of the analog readout levels. For the outermost layer these tests could be done sector-wise, once a readout group had been connected to its PCB. The PCB for the sectors of the first two layers however were mounted on the endflange of the third layer. Thus, the modules were tested individually after mounting and retested after the assembly of the three layers. In order to recover a broken module, the three layers had to be taken apart and the module and possibly also neighboring modules had to be disconnected and unscrewed. This was done several times and broken modules were either repaired or replaced.

The procedure proved very efficient. Up to 40 modules could be mounted in a day. Only three out of 768 modules were lost during the assembly, 10 modules were damaged but could be repaired and used in the final system. However, the replacement and repair of a broken module was an extremely delicate operation with a high risk of inducing more damage to the system. In the last iteration of testing at PSI, three modules $(0.35 \%)$ were found to be non-working. Figure 8.10 shows a picture of one half of the fully assembled BPIX detector.

When the BPIX detector was fully assembled a clearance test between the two half shells was performed. The test showed that the cables of the closely approaching half modules had to be slightly rearranged in order to provide enough clearance for the installation of the detector.

\subsubsection{Supply Tube Assembly}

The BPIX supply tube was fabricated at the University of Zürich between November 2007 and April 2008. The supporting elements of the supply tube structure are stainless steel cooling tubes running along the $z$-direction. They are connected to fiberglass stiffener rings and inner and outer aluminum flanges. The gaps in-between are filled with foamed material to guarantee the required rigidity. All power and slow control lines are embedded in the supply 


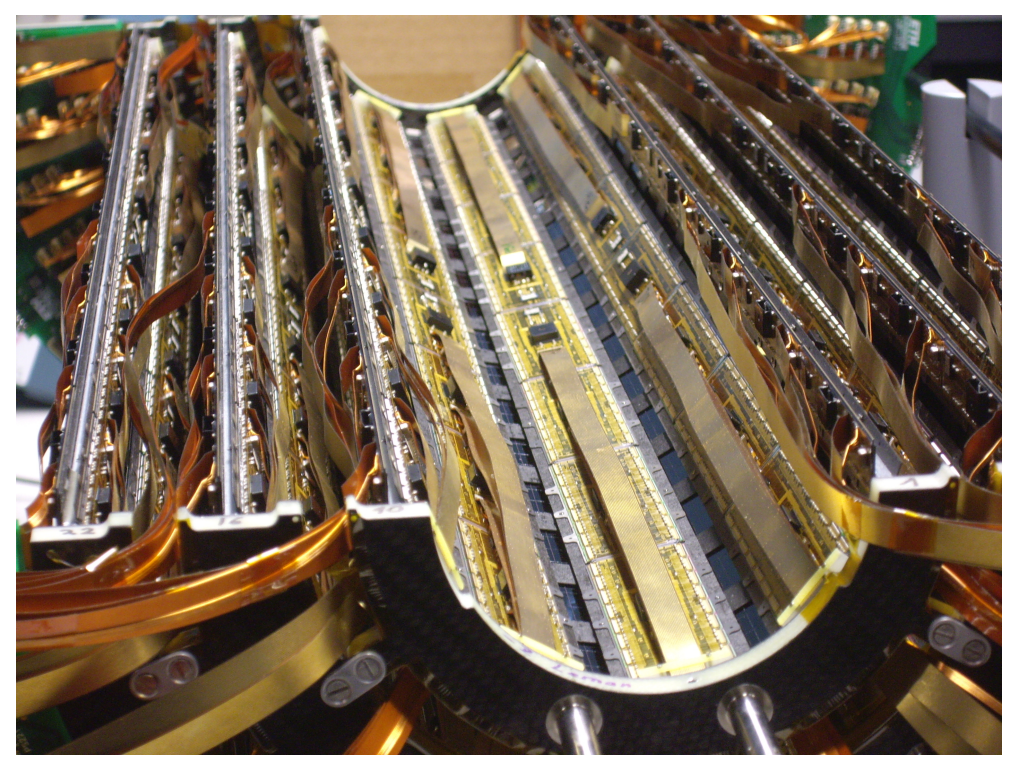

Figure 8.10: One half of the fully assembled BPIX detector.

tube body. The BPIX supply tubes are equipped with a total of 124 temperature sensors and 8 humidity sensors. The temperature sensors are placed on the CCU boards, the AOH boards and on the supply tube cooling lines.

The CCU boards were produced at the University of Zürich and thoroughly tested at PSI using the System12 setup. The proper working of the CCU ring architecture and the redundancy mechanism was checked and the programming of the PLL and the DELAY25 chip was tested. With the first version of the boards problems occurred due to weak soldering joints in the PCB which easily broke when applying mechanical stress, for instance by bending the boards. A redesign of the CCU board solved this problem. Furthermore, the temperature stability of the CCU boards was verified by successfully running them at $-20^{\circ} \mathrm{C}$ and $+55^{\circ} \mathrm{C}$. The boards proved to be reliable in long-term usage as they were operated in System12 for several months without any problems.

The detector readout electronics was integrated on the two halves of the supply tube in February and April, 2008. All components were tested for functionality before and during mounting. A standard PC with a FEC card was used to send the clock and the control commands to the CCUs, the returned digital signals were monitored on an oscilloscope and the output light intensity of the analog optical signals was measured.

In the first step, we mounted the CCU board and the analog and digital motherboards on the supply tube. We then placed the DOHs on the CCU board and connected them via optical fibers to the FEC card to establish communication and perform first functionality tests.

The mounting of the DOHs and AOHs and the inlaying of the optical fibers was done sector-wise wherefore the supply tube was placed on a rotatable mandril (figure 8.11). The main challenge was the arrangement of the 1440 single optical fibers. For logistic reasons and for the purpose of flexibility during the assembly, all AOHs and DOHs are identical and the fibers glued to the boards all have a length of $2 \mathrm{~m}$. The slack management of the fibers turned out to be very difficult as the space on the supply tube is limited and the bending radii of the fibers have to be more than $5 \mathrm{~cm}$ to guarantee maximum light transmission. At the far 
end of the supply tube optical connectors link the single fibers with fiber bundles of 12 fibers each. These bundles form pig tails with a length of about $1 \mathrm{~m}$ which then connect to the first patch panel inside CMS.

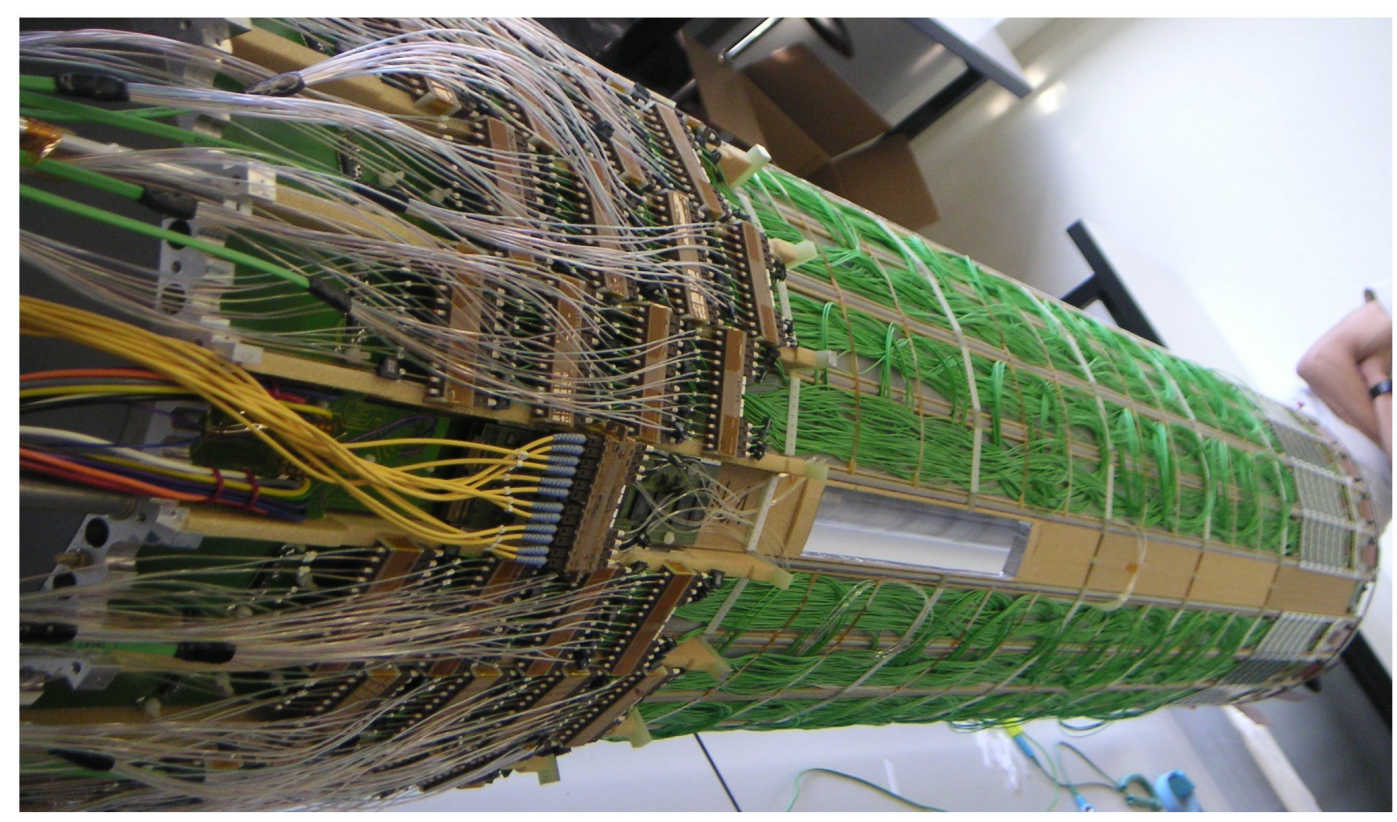

Figure 8.11: One of the four BPIX supply tubes during assembly placed on a rotatable mandril.

We first mounted and tested the DOHs and then continued with the AOHs. A $40 \mathrm{MHz}$ clock signal was injected into every DOH channel and the returned signal was checked with the help of an oscilloscope. Not a single non-working DOH channel was found. The plugging of the AOHs was more tedious as the wire-bond connections between the laser and the board were found to be very fragile. The problem was caused by a protection bar which is placed on top of the lasers and glued to the outer two lasers (see figure 8.12). This glued joint is unfortunately much stronger than the one between the lasers and the board. When bending the boards, the force is thus transferred to the wire-bonds which are likely to break. $15 \mathrm{AOHs}$ (out of 192) had to be replaced during the assembly due to broken wire-bond connections of the laser.

The stability of the analog signal is strongly affected by the undesirable temperature dependence of the AOHs. The level of the analog signal is shifted by 50 ADC counts when the temperature of the $\mathrm{AOH}$ changes by $1^{\circ} \mathrm{C}$ [116]. The FED is able to internally correct for a drift within a temperature range of $\pm 2{ }^{\circ} \mathrm{C}$. Consequently, an active cooling has to be provided to control the temperature of the AOHs within a very narrow range and assure a stable operation of the detector. For this reason, aluminum plates were placed on top of the AOHs and DOHs which connect them thermally to the supply tube cooling lines.

In a final step, the power and control cables for each sector and the central slot were mounted on the supply tube and the fibers were covered with a thin aluminum coated shielding. 


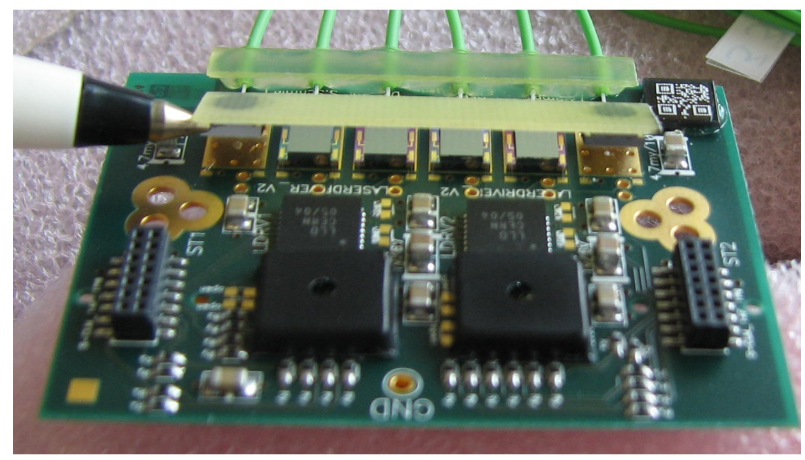

Figure 8.12: Picture of an $\mathrm{AOH}$ where the two outer lasers are disconnected. The protection bar glued to the outer two lasers is well visible.

\subsubsection{Integration of the Complete System}

The detector and the supply tubes were assembled and commissioned at PSI within only two months in May and June 2008.

The detector halves together with the supply tubes were integrated in two $5 \mathrm{~m}$ long transport boxes custom-built for the installation into CMS. Within the transport box the detector is placed on wheels on a rail system which then can be used to slide the pixel detector inside CMS. A picture of the pixel detector with the two supply tubes inside the transport box can be seen in figure 8.13 .

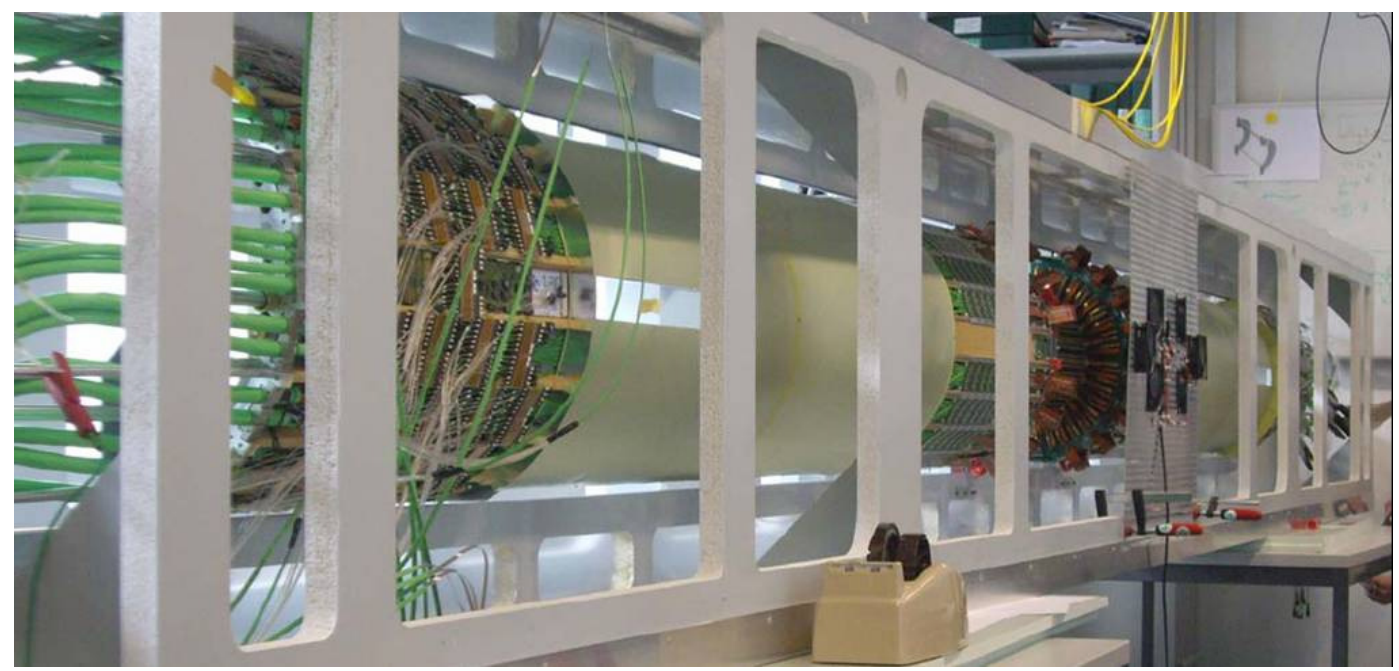

Figure 8.13: One half of the BPIX detector with the supply tubes connected. The detector system is placed on a rail system inside a $5 \mathrm{~m}$ long custom-built transport box.

The connection between the readout sectors of the supply tube and the detector endflange is provided by four Kapton signal cables and a power cable. An aluminum clamp screwed to the detector endflange retains the Kapton cables in their position. The mechanical strength of the connection between the detector and the supply tube is determined by the stiffness of the signal and power cables.

In addition to the signal and power cables, silicon rubber hoses fixed with aluminum clips 
were mounted to connect the aluminum cooling lines of the detector mechanics and of the supply tubes. A leak test of the 10 cooling lines of each half detector was performed by filling the lines and monitoring the amount of coolant. No leaks were detected inside the pixel system.

After the assembly, the complete system was fully commissioned by performing the low level hardware tests followed by detailed functionality tests. The low level hardware tests were carried out using the standalone software and could be completed within less than one day while the detailed performance tests took about 3 hours per sector.

The completely integrated system was disassembled several times for the repair or replacement of broken detector modules. In the end, the number of dead channels was found to be less than $0.4 \%$. The dead channels were due to one module without high voltage connection, two modules with a broken token passage and one module with a bad ROC header. In addition, a sector without digital readback was found and two broken $\mathrm{AOH}$ channels were observed. The former issue does not influence the analog readout and the latter was recovered by rerouting the signal through another channel.

\subsection{Installation into CMS}

The pixel detector was inserted into CMS after the installation and cabling of the silicon strip detector had been completed. The BPIX detector fits into the small volume limited by the outer radius of the beam pipe at $2.9 \mathrm{~cm}$ and the inner radius of the first layer of the strip tracker at about $21 \mathrm{~cm}$. A system with bending rails on top and bottom inside CMS had been designed to insert the pixel detector and the supply tubes along the beam pipe. A clearance of $7-8 \mathrm{~mm}$ to the beam pipe had been calculated in simulations and checked with the help of a design model. The transport box with the pixel detector was placed on an insertion table and the rail system inside the box was joint with the rail system inside CMS using temporary extension rails (see figure 8.14). In this way, the pixel detector could slide out of the transport box into its final position. At the end, the service lines were connected at the so-called patch panel 0 (PP0) to the detector infrastructure.

The detector infrastructure in the CMS cavern had to be ready well in advance of the detector installation. This included the installation and commissioning of the power supply system and the detector safety system. The cables and fibers between the electronic racks and the detector were placed in winter and spring of 2008. The connectivity of all cables and fibers was tested and measurements of the signal transmission quality were performed. The FED and FEC modules located in the CMS service room had already been installed and tested at the end of 2007.

On April 25, 2008 the commissioning system was shipped to CERN and a test installation took place. The system was craned down to the cavern through the main shaft, lifted to the installation table and inserted into CMS. The installation of the commissioning system went smoothly and was finished within less than four hours. The power cables and the optical fibers of the equipped sector were connected at the PP0 and the correct cable lengths were verified. The installation test did not reveal any need for mechanical adjustment before the final installation.

The two halves of the BPIX final detector system were transported to CERN on July 15, 2008. After the transport, we tested the system in the surface hall at P5 and no additional damage was found. The installation of the final system started on July 23. Both halves of 


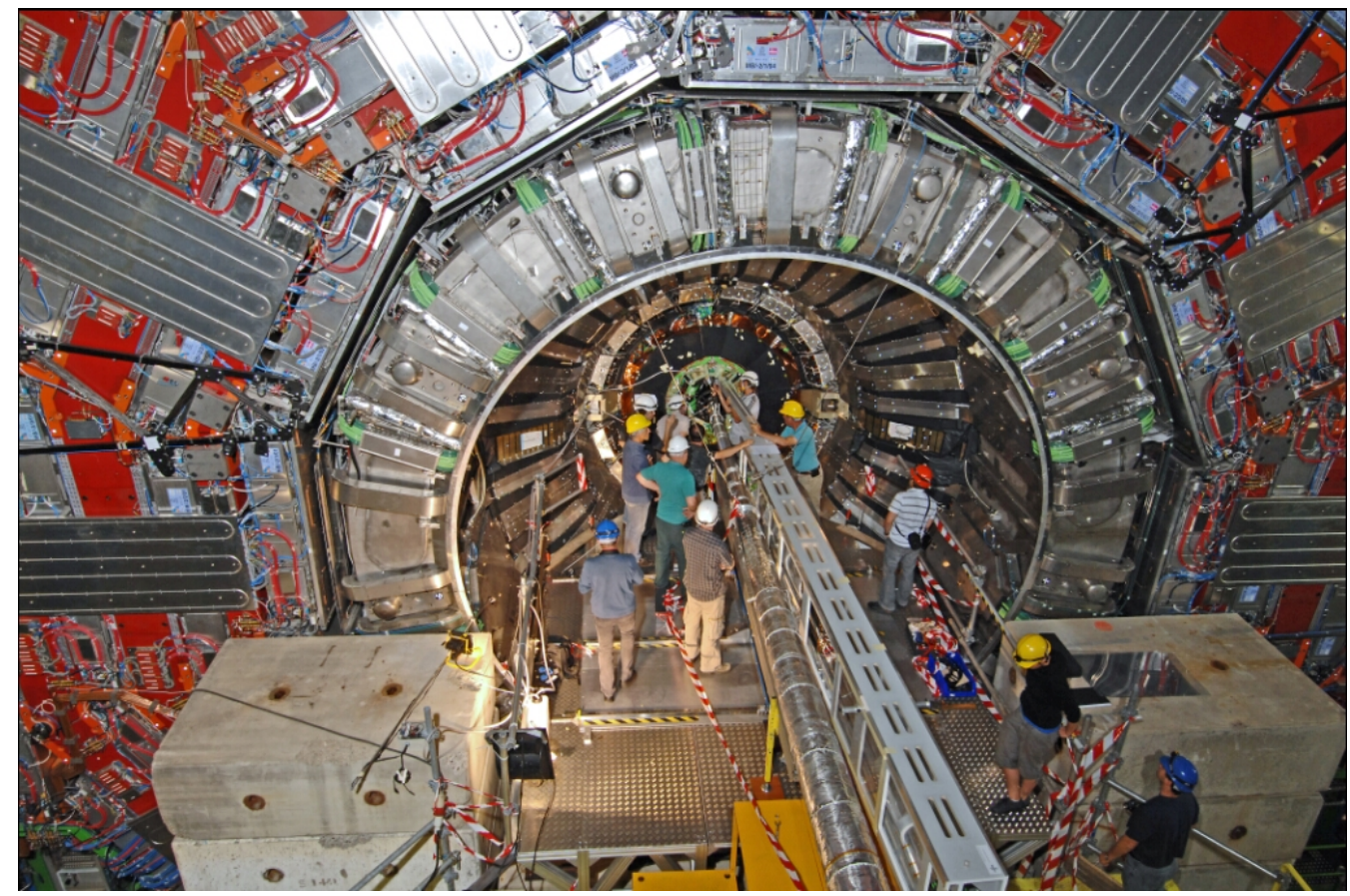

Figure 8.14: Insertion of the BPIX detector inside CMS. In the picture, the BPIX detector is inside the transport box which is placed on the insertion table next to the beam pipe. The rail system inside CMS and the transport box are joint by extension rails.

the detector were lowered into the cavern on the same day. The insertion of the inner shell was completed without any problems and all the connections were made, a total of 40 power and control cables and 18 multi-fiber ribbons. In order to make a fast check-out possible a temporary cooling system was set up. The second half of the BPIX detector was inserted the following day. The first attempt failed due to a collision of the detector end-flanges of the two detector halves. This problem was solved by mechanically modifying the suspension of the insertion wheels to enlarge clearance between the two half shells, and allowed us to finalize the insertion successfully.

All power and control cables and all optical fibers of the BPIX detector were connected by July 24. A picture of the detector in the final position and the connection area PP0 is shown in figure 8.15. The BPIX services had to be disconnected again when the final cooling tubes were joined and when the forward pixel detector was installed.

The cooling lines of the pixel supply tube are connected to the cooling system using stainless steel flexible pipes. In the first running period after the installation the cooling fluid had a temperature of $+17^{\circ} \mathrm{C}$ and the 10 barrel pixel cooling loops did not show any leak.

\subsection{Commissioning and Performance}

The testing of the pixel system started as soon as the pixel services were connected and cooling was available. The low level hardware tests were done first to quickly evaluate the performance of all detector components. On July 29, all sectors of the BPIX detector were working satisfactorily so that the construction of CMS could proceed with the installation 

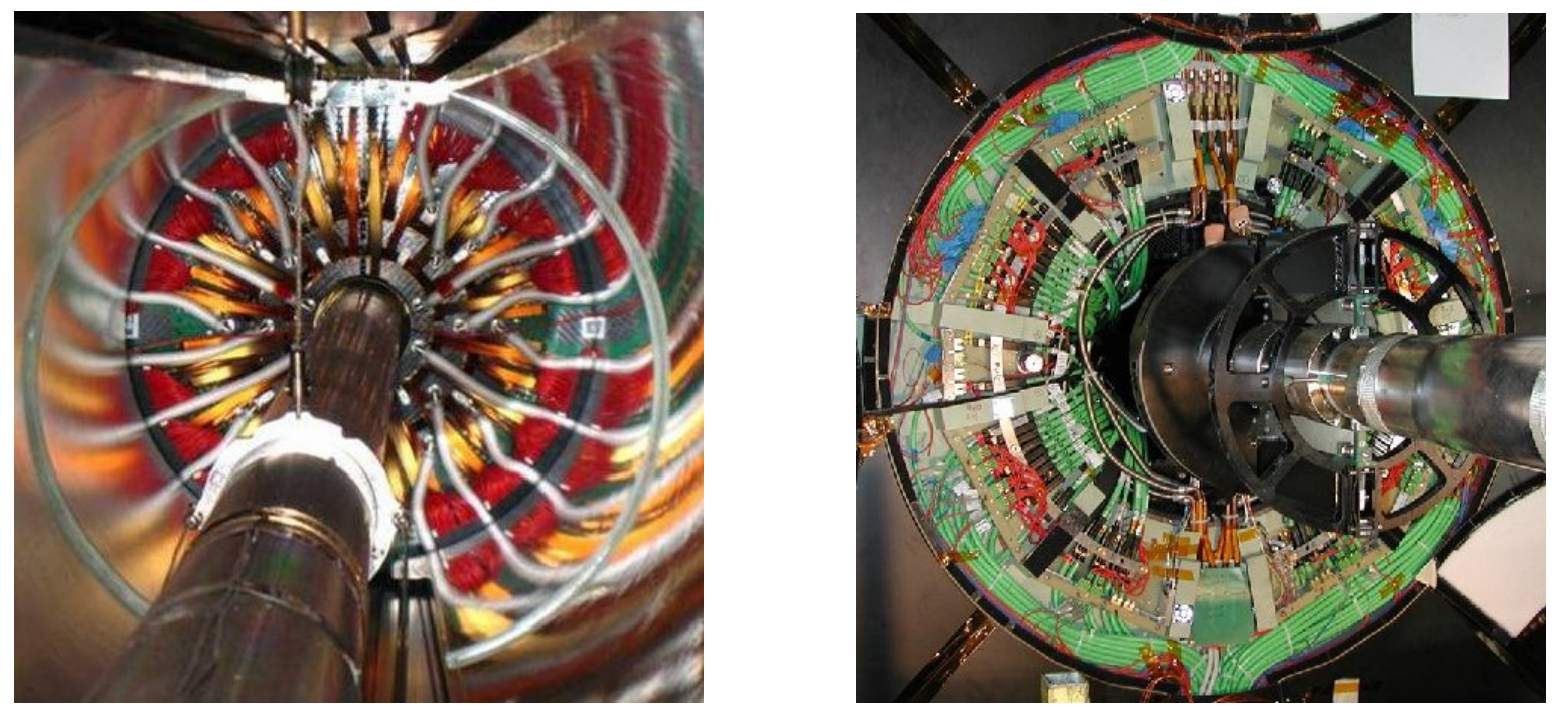

Figure 8.15: View of the BPIX detector in its final position. Left: The picture shows the BPIX detector inside CMS enclosing the central beam pipe. The camera is placed inside the detector supply tube and the detector endflange covered by the cooling lines and signal and power cables can be seen. Right: Picture of the connection area PP0 at the far end of the pixel volume. The connection of power cables (green cables), optical fibers (wrapped in red rubber bands) and cooling lines are visible. In the foreground the beam pipe suspension is shown.

of the forward pixel detector. On August 7, the closing of the CMS detector started and thus access to the pixel detector was no longer possible. A period of extensively testing and calibrating the detector before the first cosmic data taking took place in October and November 2008. The results of the testing and the performance of the BPIX detector are reviewed in the following.

\subsubsection{Performance of the Optical Links}

The optical signals pass four connection points before they are translated into electronic signals in the FED modules in the service room: a single-fiber (MU) connection on the supply tube located at $2 \mathrm{~m}$ distance from the laser, a multi-fiber (ribbon) connection at the pixel detector PP0 at a distance of $3 \mathrm{~m}$, a multi-ribbon connection at the strip tracker PP1 at $7 \mathrm{~m}$ distance and a connection to the FED modules at $63 \mathrm{~m}$ distance. The latter connection at the strip tracker end-flange was not accessible anymore at the time of the BPIX installation.

The digital-optical ring is tested by sending and receiving a $40 \mathrm{MHz}$ clock signal. For the analog-optical lines the scan of the laser bias range was repeated. After three iterations of re-cleaning the PP0 connections, the optical fibers for the transmission of the digital signal showed an excellent performance while 29 out of 96 ribbons of the analog readout contained noisy fibers. These 29 ribbons were investigated with the help of an optical reflectometer (OTDR) and a visual inspection of the connection to the FED. The OTDR measurement did not show any reflection at the PP0 connections which means that an optimum light transmission was provided. However, in 19 cases a reflection peak at a MU connection was spotted. As the MU connectors are located on the supply tube, it was not possible to improve 
the connection by cleaning. In 11 cases this reflection influences either the noise or the slope of the calibration curve for the corresponding channel. Fortunately, further testing indicates that this does not degrade the performance of the analog address level decoding substantially.

In addition, a bad PP1 connection was found. The four fibers passing through that connection could be recovered by using a spare ribbon. An example of the OTDR measurement of a fiber with a reflection at the MU and the PP1 connection is shown in Figure 8.16.

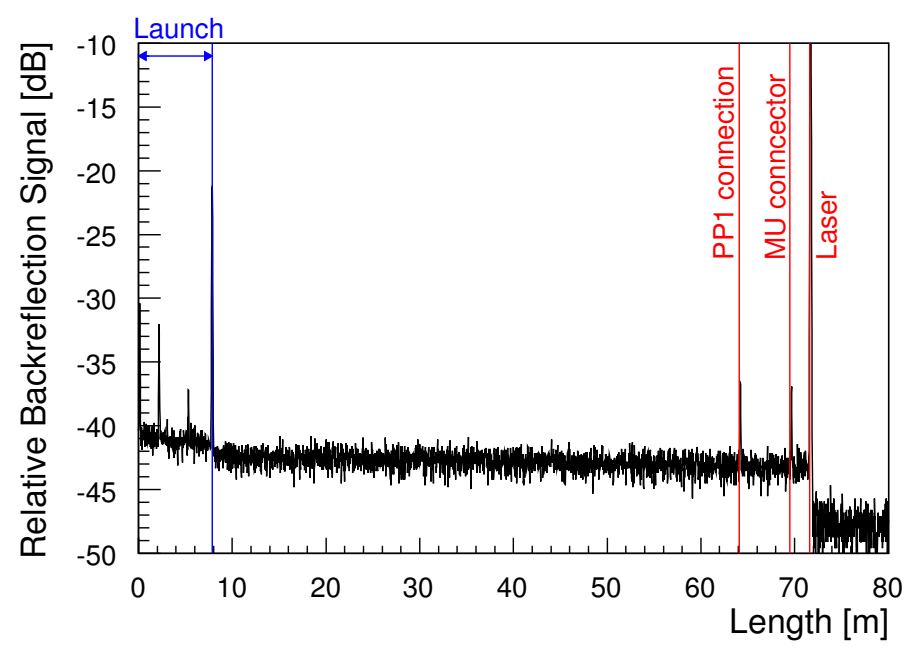

Figure 8.16: OTDR measurement of a fiber with a reflection peak at the MU and the PP1 connection.

The visual inspection of the connection to the FED was performed with a microscope and 19 ribbons with marks on the fibers were spotted. In about $50 \%$ of the cases cleaning was successful and slightly improved the noise behavior. A microscopic view of the FED connection with marks on the fibers can be seen in Figure 8.17.

Not a single optical fiber of the BPIX detector was lost during all operations.
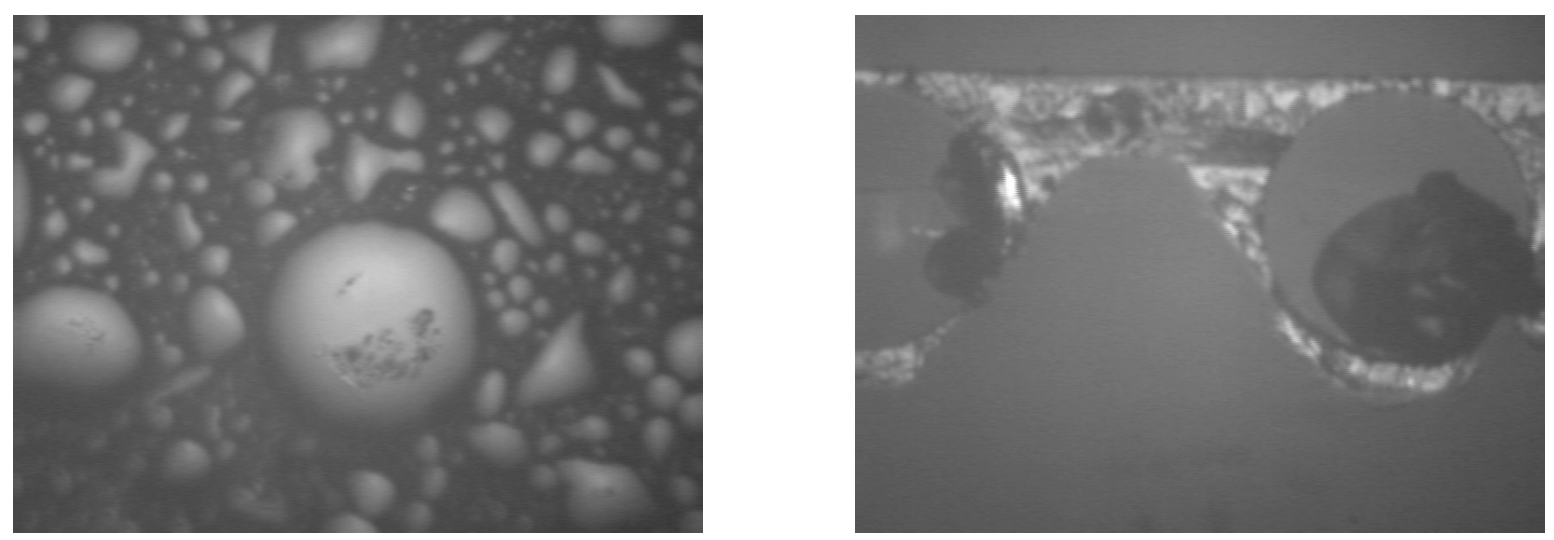

Figure 8.17: Microscope picture of the optical connection at the FED. On the left hand side a single optical fiber is shown, while on the right hand side two optical lines inside the FED connector are visible. All fibers are partially covered by dirt. 


\subsubsection{Detector Module Functionality}

Although the testing after the transport of the BPIX detector has not revealed any damage (in addition to the four modules known to be non-working), after the installation five more broken modules were identified. The failure modes are:

- 3 modules without high-voltage connection,

- 1 module with a bad ROC header,

- 1 module that could not be programmed.

Furthermore, 4 individual ROCs did not produce valid signals. An additional module with a bad TBM header was recovered by rerouting the signal through the other TBM. In total 100 ROCs $(0.87 \%)$ of the BPIX detector could not be operated, $80 \%$ of the failure modes were explained by broken wire bonds or missing high voltage connection, the remaining $20 \%$ were due to modules which did not respond to programming and thus had to be disabled. The number of dead pixels on otherwise functional ROCs is very low $(0.01 \%)$. These failures are caused by faulty bump-bond connections between the ROC and the sensor and have already been observed during module testing.

\subsubsection{Results of the Detector Calibration}

In this section the results of the BPIX detector testing and calibration period in summer and autumn 2008 are summarized. More details can be found in [120]. The sequence of tests introduced in section 8.2 were performed. After determining a working region for each module and adjusting the analog levels in the AOHs, front-end chips and FED channels, an address level calibration for each ROC was run. The quality of the address encoding is evaluated by comparing the width of the address peaks to the separation between to neighboring pixels. The result is shown in figure 8.18. The level separation is considerably larger than the width of the peaks, even in comparison with the broadest peaks. The few channels affected by dirty optical connectors have a smaller level separation, however still large enough for a reliable address decoding. The address level calibration is temperature dependent and has to be repeated every time the operating conditions change.

The threshold values for the BPIX modules were programmed to the target values determined in the module testing. Afterwards the threshold and the noise were measured using the S-Curve method. An average threshold of 3829 electrons was found, well above the noise level at 141 electrons.

The results of the analog pulse height calibration are given in figure 8.19. The gain and pedestals are obtained from a linear fit. The tail in the pedestal distribution is due to misconfiguration of individual ROCs which was recovered at a later stage of detector calibration.

\subsubsection{Results of the Cosmic Run}

The goal of the cosmic data taking with the CMS detector is the commissioning and calibration of the individual subdetectors, the alignment of the tracking detectors and the muon chambers and the testing of the DAQ system. 

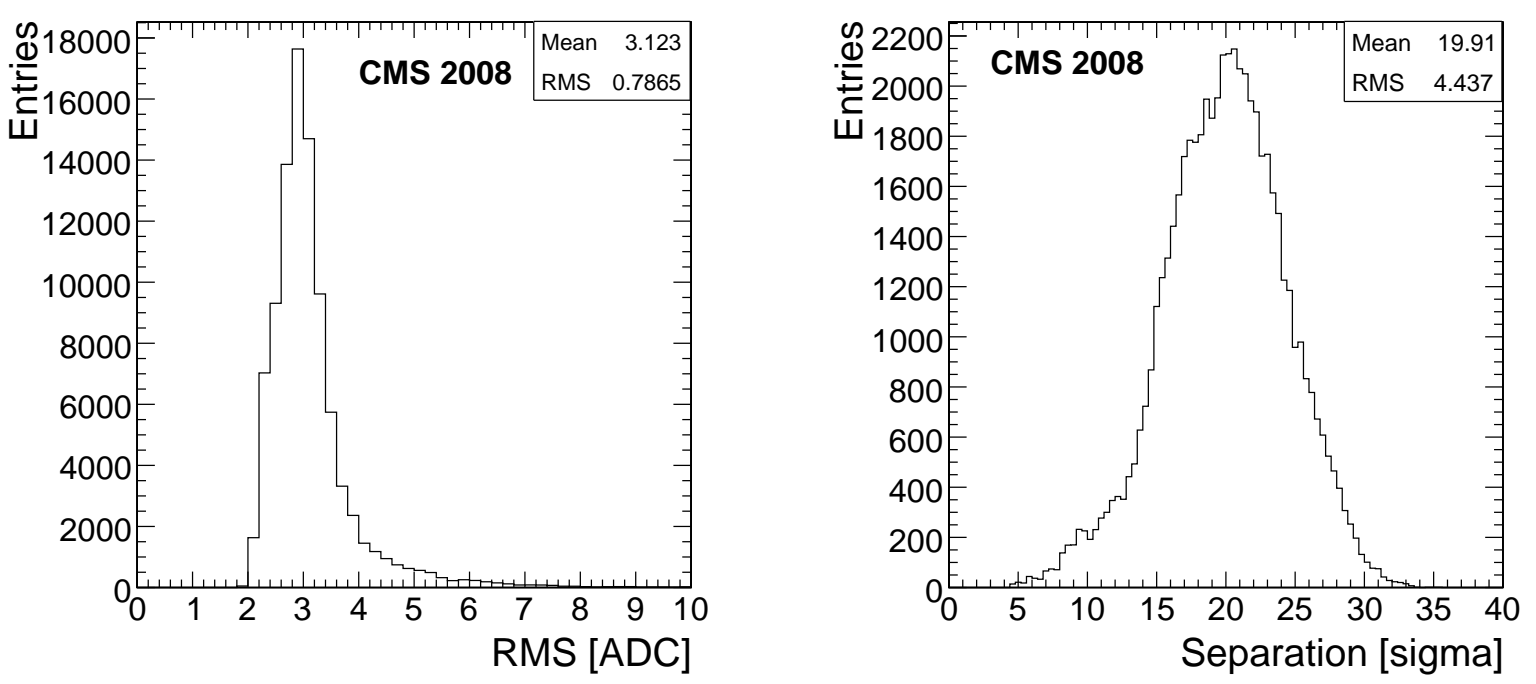

Figure 8.18: Results of the address level calibration [120]. Left: RMS of width of the six address level peaks for all operable ROCs in the detector. Right: Separation between the mean of two neighboring peaks. The separation is given in units of sigma, defined by summing in quadrature the RMS widths for adjacent peaks.

The pixel detector showed a very good performance during cosmic data taking and proved its ability for stable running. The main cause of interruption during the pixel detector operation was problems with the cooling plant or the power supply. Together with some rare failures of the detector control software this amounted to an overall data taking efficiency of $97 \%$.

The CMS experiment recorded about 270 million cosmic-ray triggered events with the solenoid at a field strength of $3.8 \mathrm{~T}$. More than 4 million tracks were reconstructed out of which approximately 85000 did cross the pixel detector volume. An event display showing a cosmic muon traversing the pixel detector is shown in figure 8.20

The results of the alignment of the silicon strip and pixel detectors is detailed in [94]. After performing a track-based alignment, the precision of the detector position with respect to particle trajectories had been derived from the distribution of the median of the cosmic muon track residuals measured in each module. A precision of $3 \mu \mathrm{m}$ in the $r \phi$ direction and $4 \mu \mathrm{m}$ in the $z$ direction has been achieved for the BPIX detector.

In total about 257000 hits were reconstructed in the pixel detector with an average number of 60 hits per ROC. In figure 8.21 the number of track-associated hits in each BPIX ROC is shown. The ROCs that did not have any hits were excluded from the readout for the reasons given in section 8.5.2.

Furthermore, the cosmic data were used to estimate the hit efficiency of the BPIX modules from data. This is done by extrapolating tracks reconstructed in the silicon tracker to the pixel detector and checking the presence of a compatible hit. The efficiency is then defined as the number of tracks with a compatible pixel hit divided by the total number of tracks. For the efficiency measurement only tracks to which one additional hit was associated in both the top and bottom half of the pixel detector were selected. A layer efficiency averaged over the operable modules of $(97.1 \pm 1.4) \%,(97.1 \pm 1.9) \%$ and $(96.4 \pm 2.6) \%$ was measured for the first, second, and third barrel layers, respectively. 

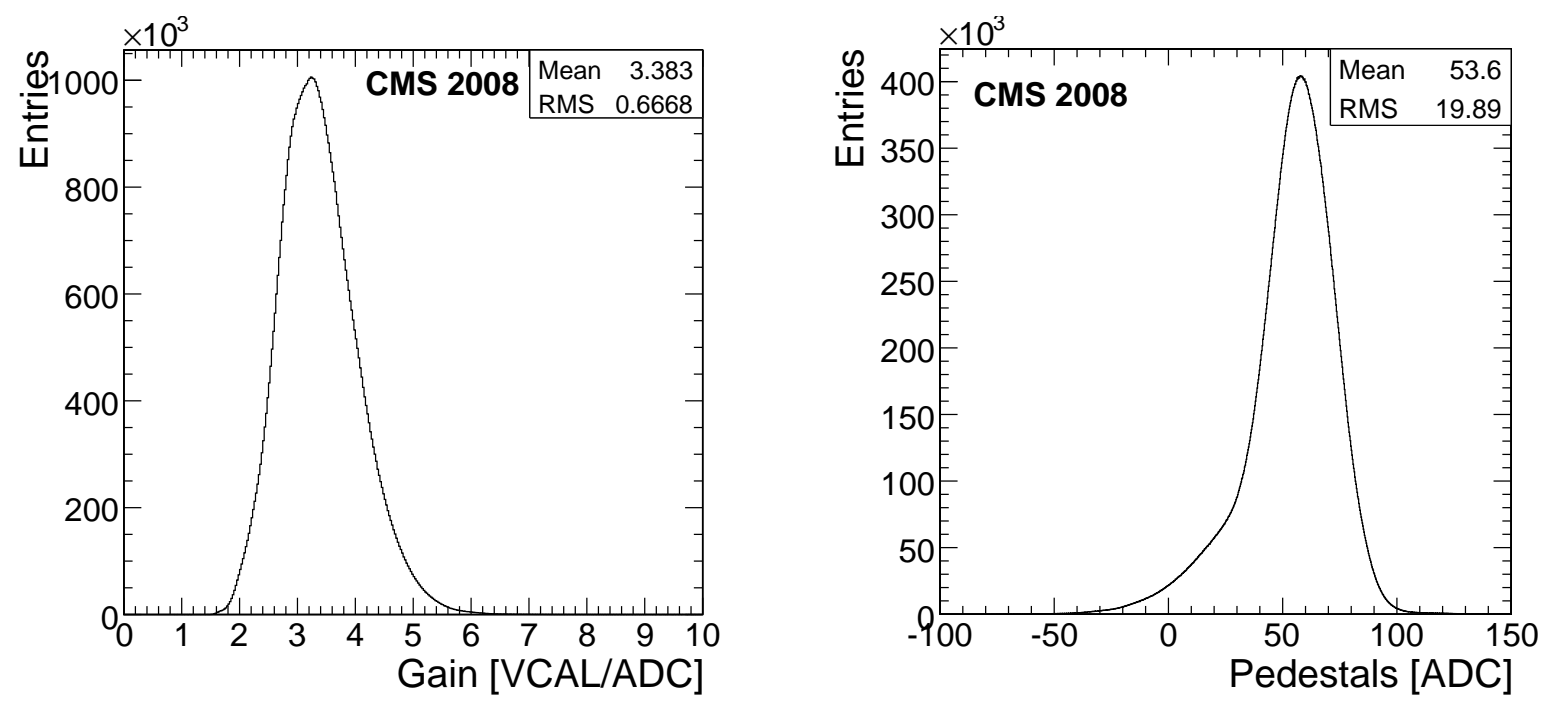

Figure 8.19: Distribution of gain (left) and pedestals (right) for all pixels extracted from the linear fit to the pulse height curve [120].

\subsection{Summary}

The contributions to the construction and commissioning of the CMS pixel barrel detector made during this thesis were presented in this chapter. The construction of the BPIX detector included the mounting of the modules on the mechanical cooling structure, the assembly of the supply tube and the integration of the complete system.

A slice of the CMS control and data acquisition system was established at PSI in order to gain experience in operating the complete system and fully commission the BPIX detector before transporting it to CERN. A sophisticated testing procedure has been developed and implemented in a standalone software framework. This procedure allows to verify the functioning of the main detector components in a short period of time and was therefore of utmost importance for the testing phase after the installation at CERN.

The installation was completed within only a few days and the first test of the BPIX detector revealed an excellent performance with less than $1 \%$ dead channels.

After a period of extensively testing and calibrating the pixel detector at CERN, the first data taking of cosmic data took place in October and November, 2008. During this period the detector was running stable with a high data taking efficiency. 


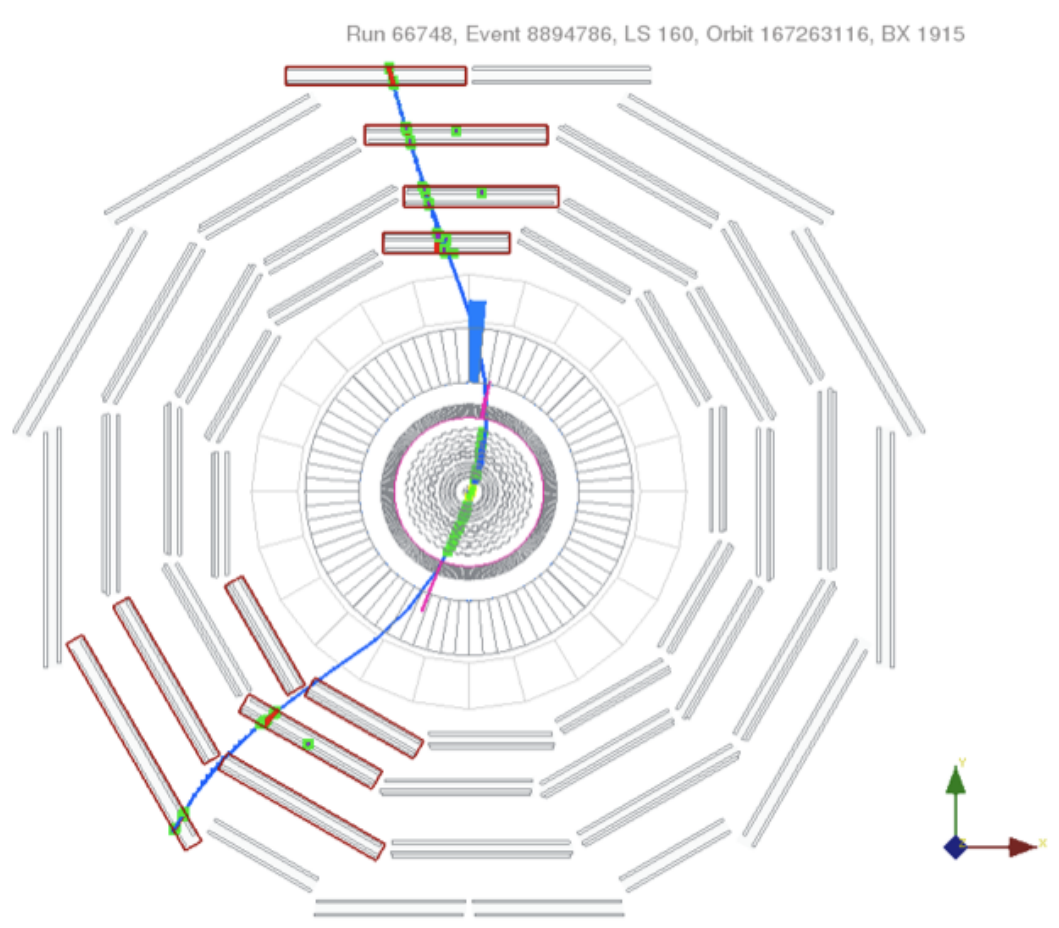

Figure 8.20: CMS event display showing a cosmic muon traversing the pixel detector.
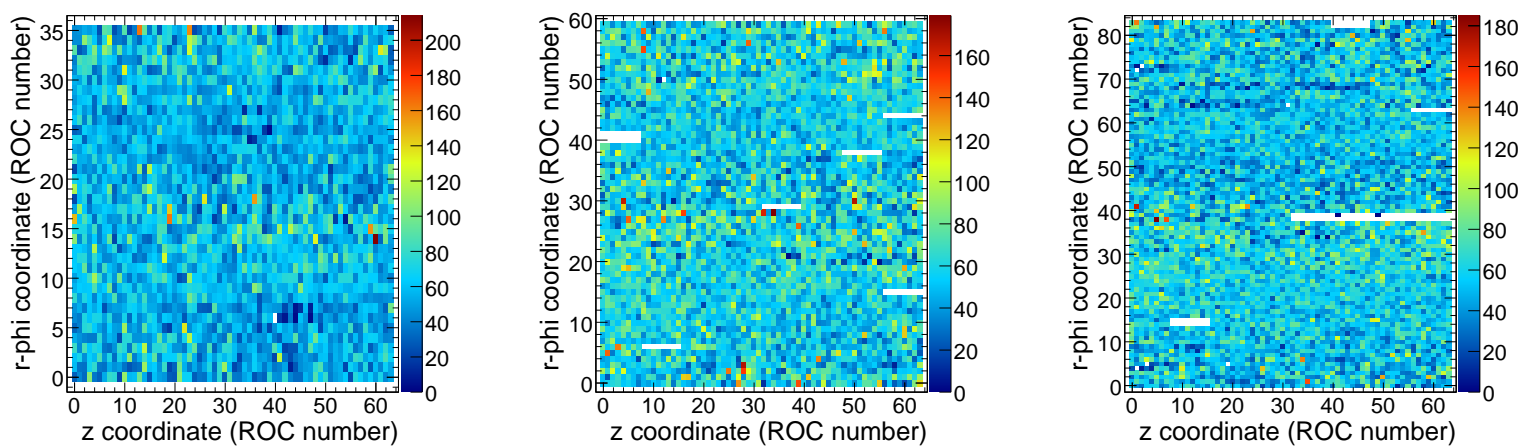

Figure 8.21: Number of hits associated to a track detected in each ROC for the first (left), second (middle) and third (right) barrel layers [120]. Bins in white correspond to readout chips excluded from data taking. 


\section{Chapter 9}

\section{Conclusion and Outlook}

A study of the inclusive $b$-quark production at the CMS experiment has been presented within this work. Thanks to the large $b$-quark production cross section at the LHC, high statistics data samples are available soon after the LHC startup. The measurement of the $b$-quark production cross section with these data is therefore a prime candidate to yield one of the first physics result obtained from proton-proton collisions at a center-of-mass energy of $\sqrt{s}=7 \mathrm{TeV}$.

As a result of this thesis, an analysis strategy has been developed which focusses on the reconstruction of muons originating from semileptonic decays of $b$-quarks. The fraction of signal events in data is determined on a statistical basis by performing a fit to the measured $p_{\perp}^{\text {rel }}$ distribution by means of simulated templates for signal and background events.

The $p_{\perp}^{\text {rel }}$ variable is defined with respect to the fragmentation jet direction reconstructed from charged particles tracks only. With this technique an efficient reconstruction of the jet direction is possible even for very low-energy jets.

The event selection and the performance of the physics object reconstruction has been studied in collision data and simulation and in general a remarkably good agreement is found.

A first measurement of the inclusive $b$-quark production cross-section at a center-of-mass energy of $\sqrt{s}=7 \mathrm{TeV}$ has been presented. The measurement is based on data statistics corresponding to an integrated luminosity of $\mathcal{L}=8.1 \mathrm{nb}^{-1}$. The data has been recorded by the CMS experiment during the first months of data taking in April and May, 2010.

The preliminary result for the total inclusive $b$-quark production cross-section in the visible kinematic range is

$\sigma\left(p p \rightarrow b+X \rightarrow \mu+X^{\prime}, p_{T}^{\mu}>6 \mathrm{GeV},\left|\eta^{\mu}\right|<2.1\right)=\left(1.48 \pm 0.04_{\text {stat }} \pm 0.22_{\text {syst }} \pm 0.16_{\text {lumi }}\right) \mu \mathrm{b}$

Furthermore, a measurement of the differential b-quark production cross-section as a function of muon transverse momentum and pseudorapidity was performed. The measurement constitutes a sensitive probe of the predictions of perturbative QCD at the unprecedented high energy scale provided by LHC. It was compared to the leading order and next-to-leading order MC predictions. The data tends to be higher than the MC@NLO prediction at low transverse momentum and central rapidity.

In the second part of this thesis, the hardware related work has been presented. The integration of the CMS pixel barrel detector has been accomplished within about two years. The availability of a test stand at PSI has proven particularly important for commissioning 
the individual detector components as well as for operating the final detector system. It allowed to transport the detector in a fully functional state to CERN and to install it into CMS within the tight schedule.

A tremendous joint effort in commissioning and calibrating resulted in the successful and stable operation of the CMS pixel detector during cosmic and collision data taking.

The CMS pixel detector has demonstrated excellent performance and is going to play a key role in tracking based physics analyses like the $b$-quark production measurement presented in this work. 
Appendix A

Maximum Likelihood Fits 


\section{A.1 Bins in Muon Transverse Momentum}

\section{A.1.1 Fit Result}
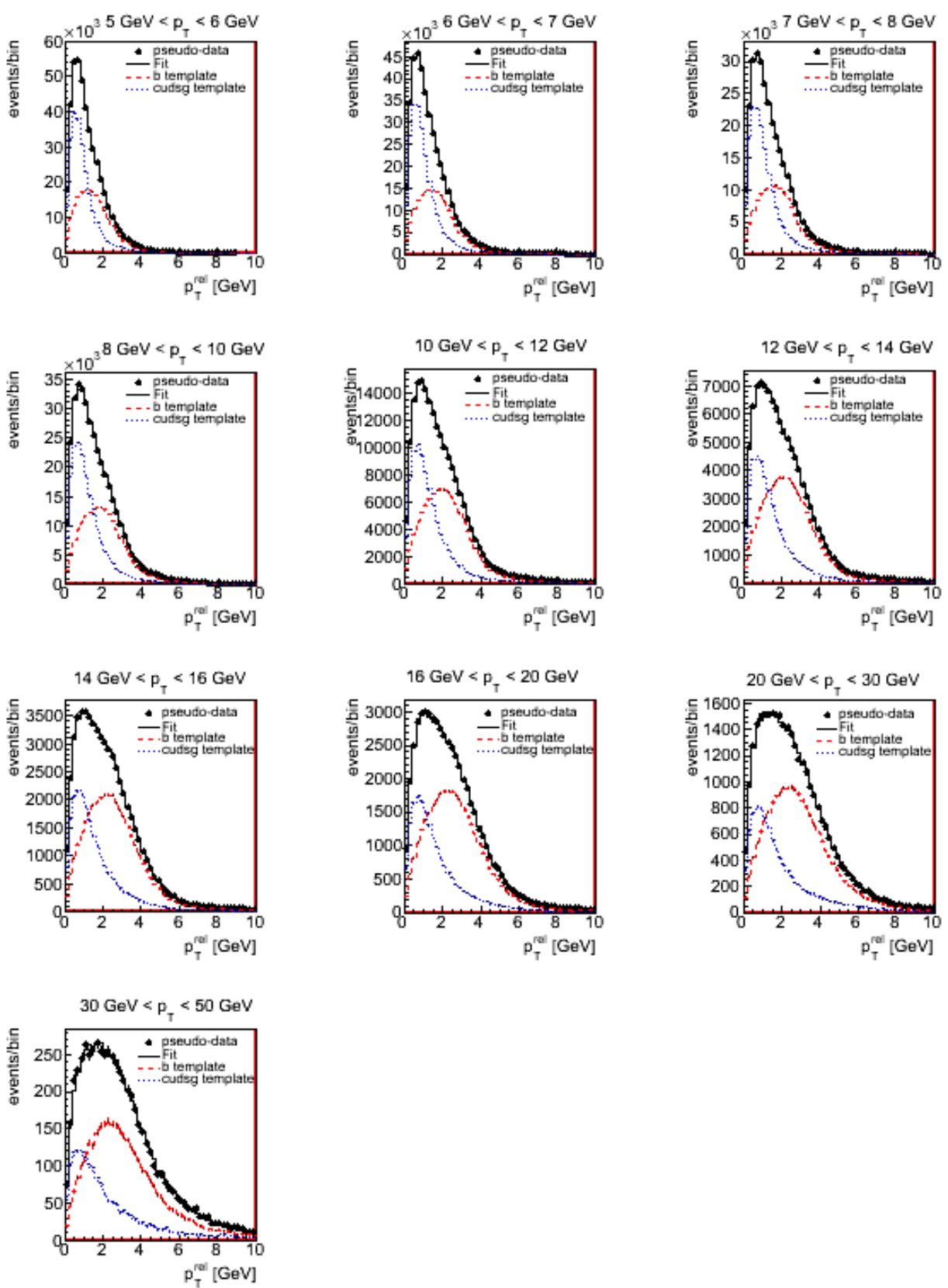

Figure A.1: Fit result obtained by dividing the MC sample into two independent subsamples and using the approximate fitting method. The red dashed and the blue dotted line are the $b$ and cudsg-template, respectively. The black full circles correspond to the data distribution, while the black line is the result of the fitting procedure. The "data" distribution is scaled to an integrated luminosity of $1 \mathrm{pb}^{-1}$. All bins in muon transverse momentum are shown. 


\section{A.1.2 Fit Deviation in the Approximate Method}
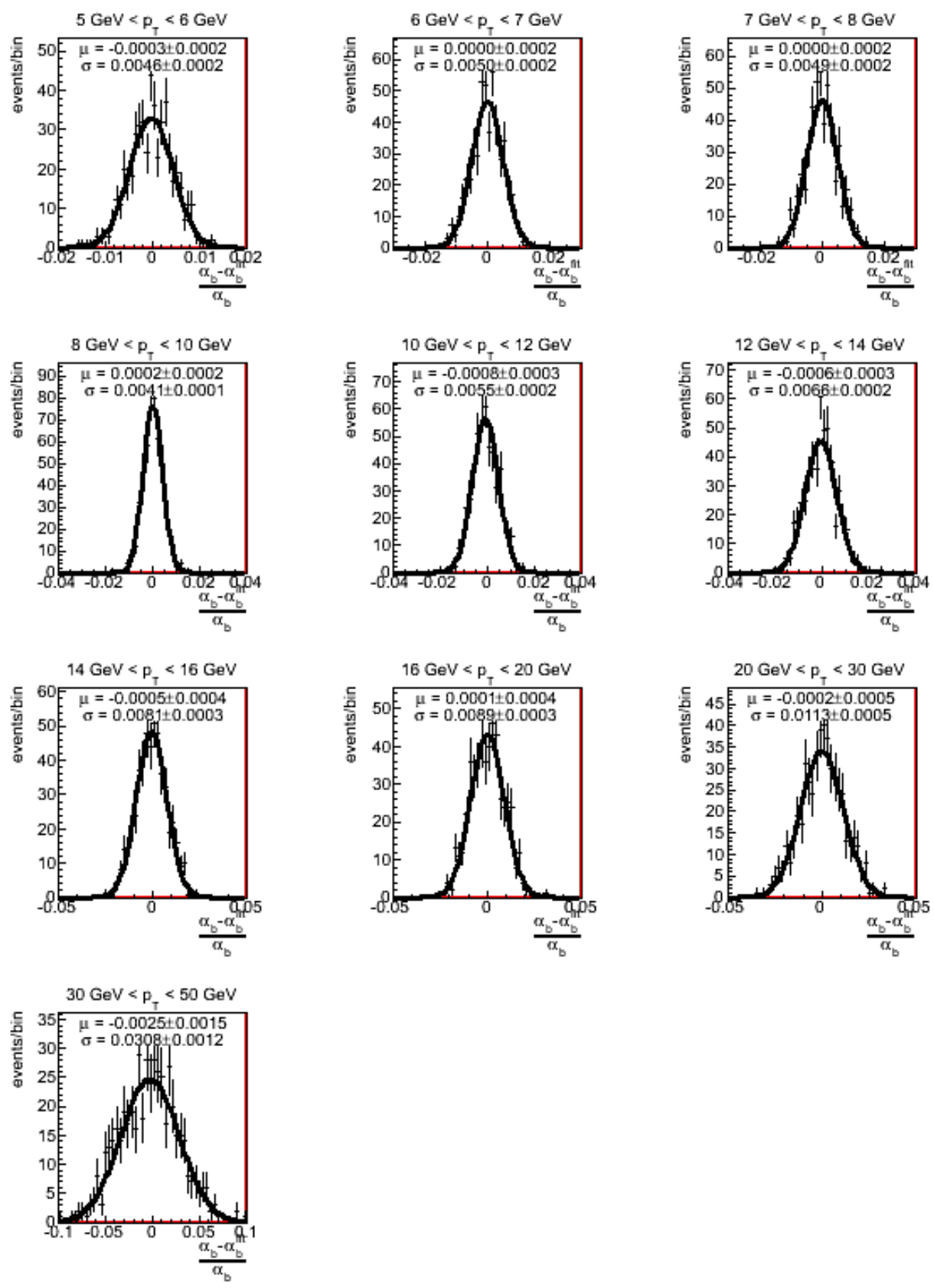

Figure A.2: Deviation of the fitted scale factor $\alpha_{b}^{f i t}$ in $b$-events from the true value $\alpha_{b}$ when using the approximate fitting method. The results are obtained from repeated pseudo experiments with data statistics generated by appropriate random variations corresponding to an integrated luminosity of $1 \mathrm{pb}^{-1}$. All bins in muon transverse momentum are shown. 


\section{A.1.3 Pull Distributions of the Approximate Method}
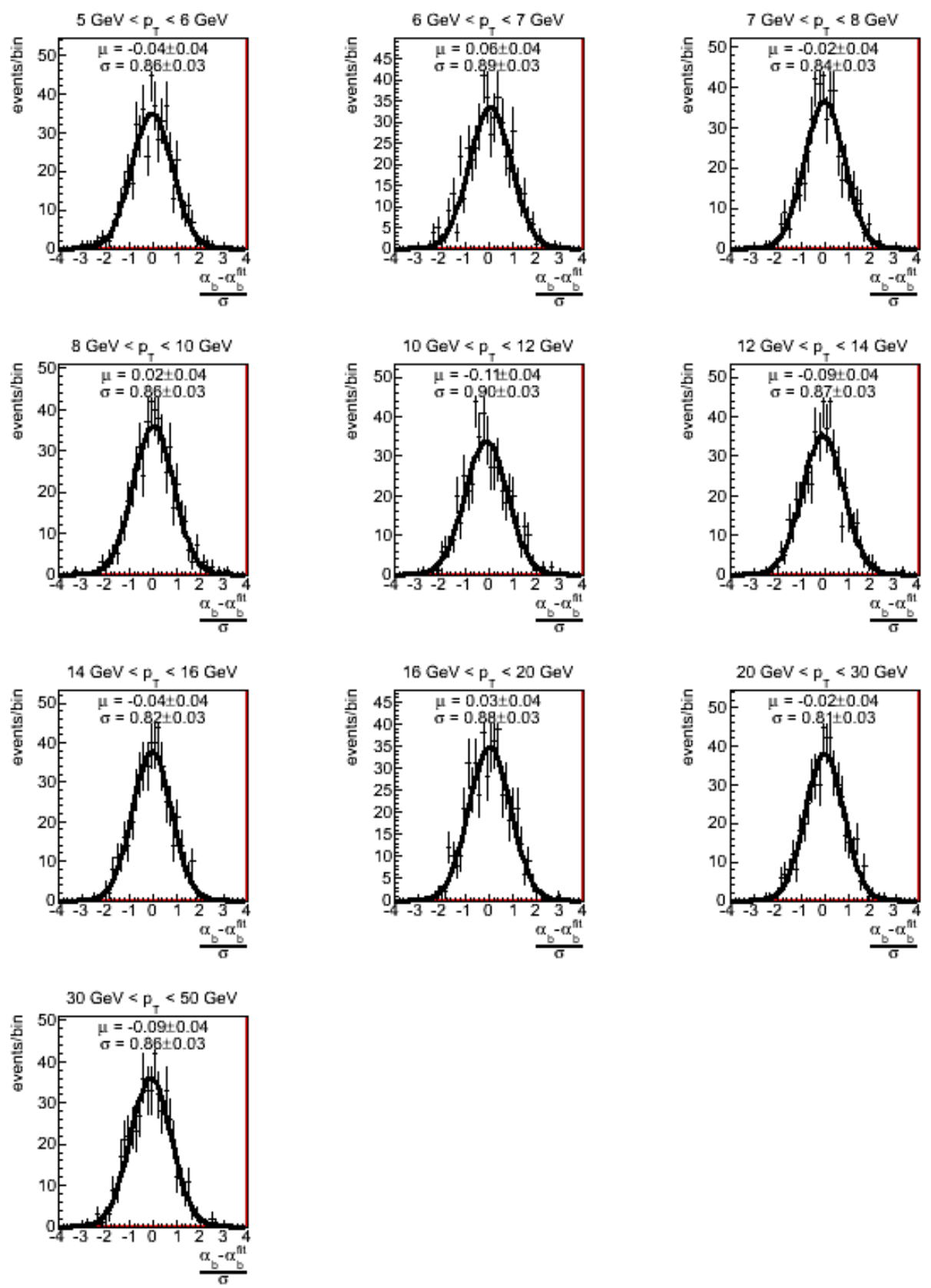

Figure A.3: $b$-fraction pull distributions resulting from the approximate fitting method. The pull distributions are obtained from repeated pseudo experiments with data statistics generated by appropriate random variations corresponding to an integrated luminosity of $1 \mathrm{pb}^{-1}$. All bins in muon transverse momentum are shown. 


\section{A.1.4 Fit Deviation in the Full Treatment}
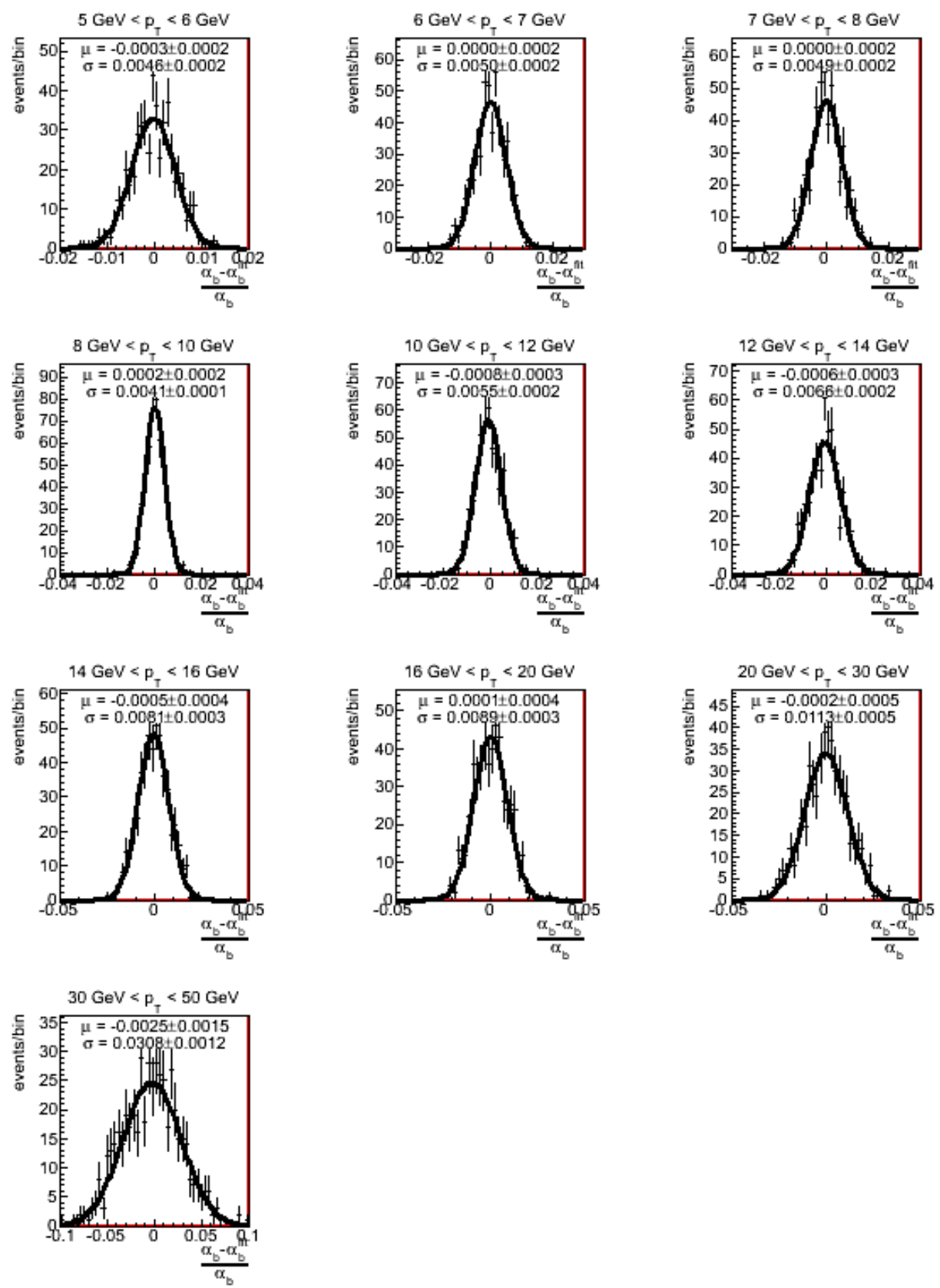

Figure A.4: Deviation of the fitted scale factor $\alpha_{b}^{\text {fit }}$ in $b$-events from the true value $\alpha_{b}$ when using the full treatment in the fitting procedure. The results are obtained from repeated pseudo experiments with data statistics generated by appropriate random variations corresponding to an integrated luminosity of $1 \mathrm{pb}^{-1}$. All bins in muon transverse momentum are shown. 


\section{A.1.5 Pull Distributions of the Full Treatment}
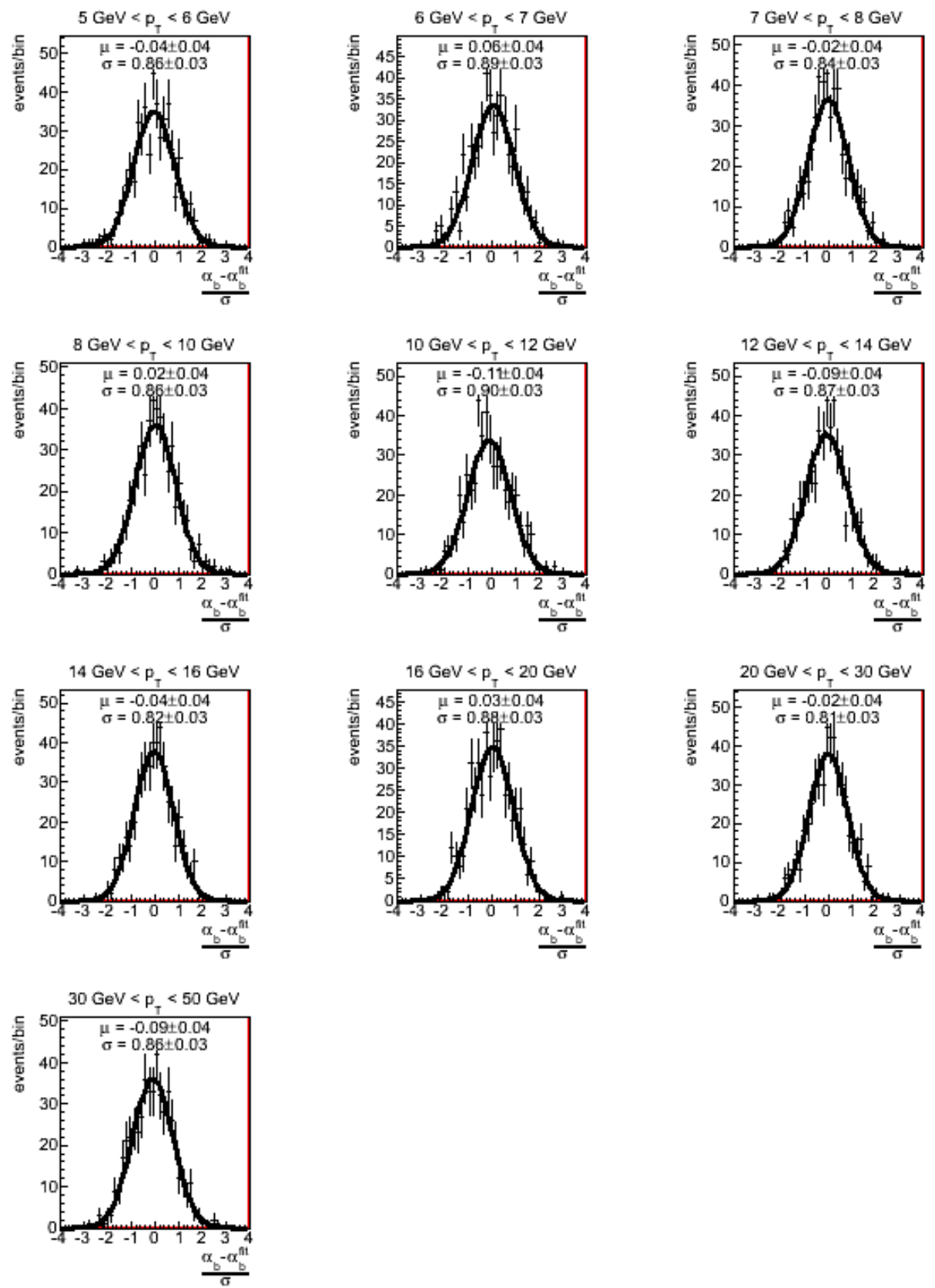

Figure A.5: $b$-fraction pull distributions resulting from the full treatment in the fitting procedure. The pull distributions are obtained from repeated pseudo experiments with data statistics generated by appropriate random variations corresponding to an integrated luminosity of $1 \mathrm{pb}^{-1}$. All bins in muon transverse momentum are shown. 


\section{A.2 Bins in Muon Pseudorapidity}

\section{A.2.1 Fit Result}
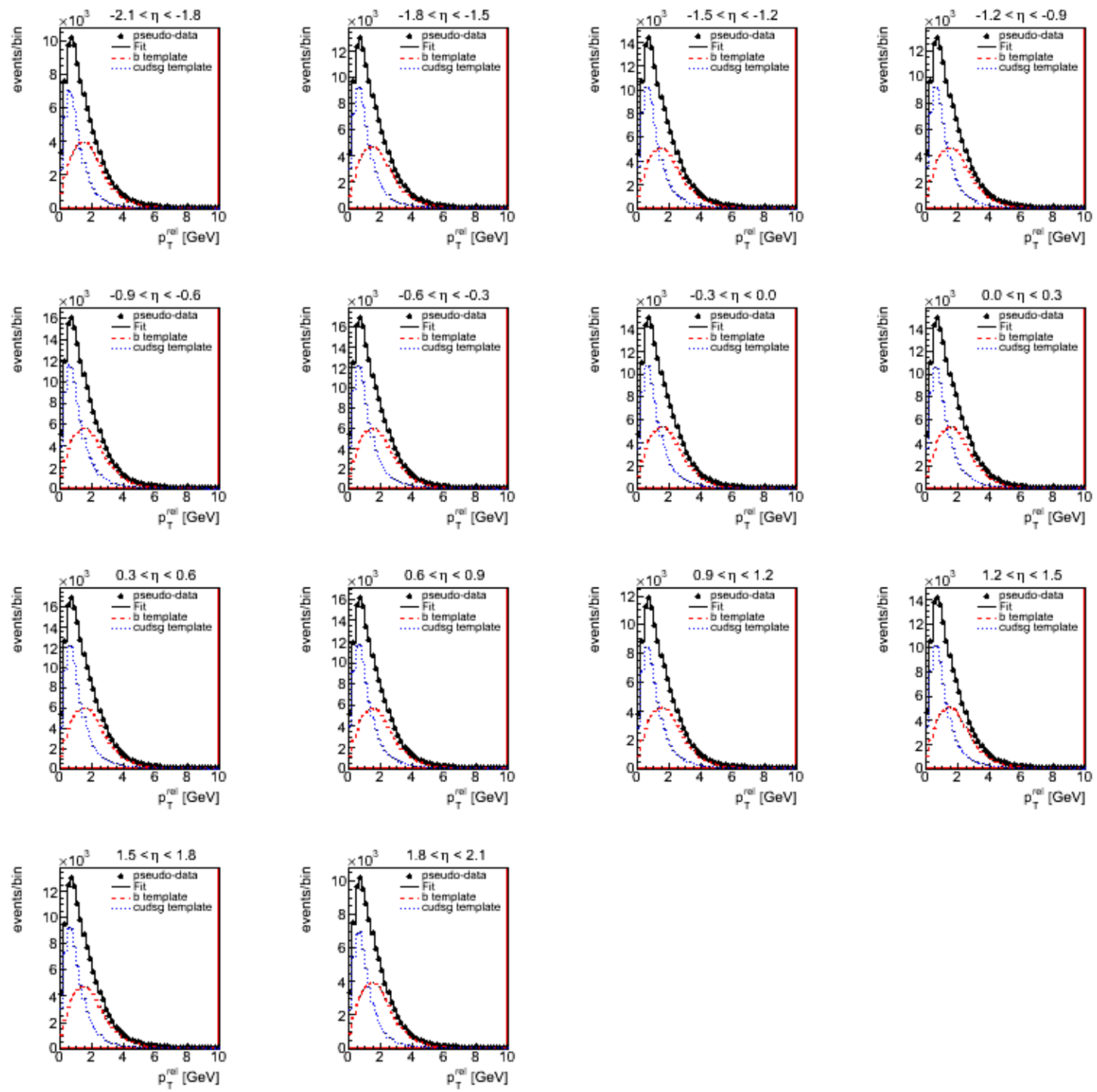

Figure A.6: Fit result obtained by dividing the MC sample into two independent subsamples and using the approximate fitting method. The red dashed and the blue dotted line are the $b$ and cudsg-template, respectively. The black full circles correspond to the data distribution, while the black line is the result of the fitting procedure. The "data" distribution is scaled to an integrated luminosity of $1 \mathrm{pb}^{-1}$. All bins in muon pseudorapidity are shown. 


\section{A.2.2 Fit Deviation in the Approximate Method}
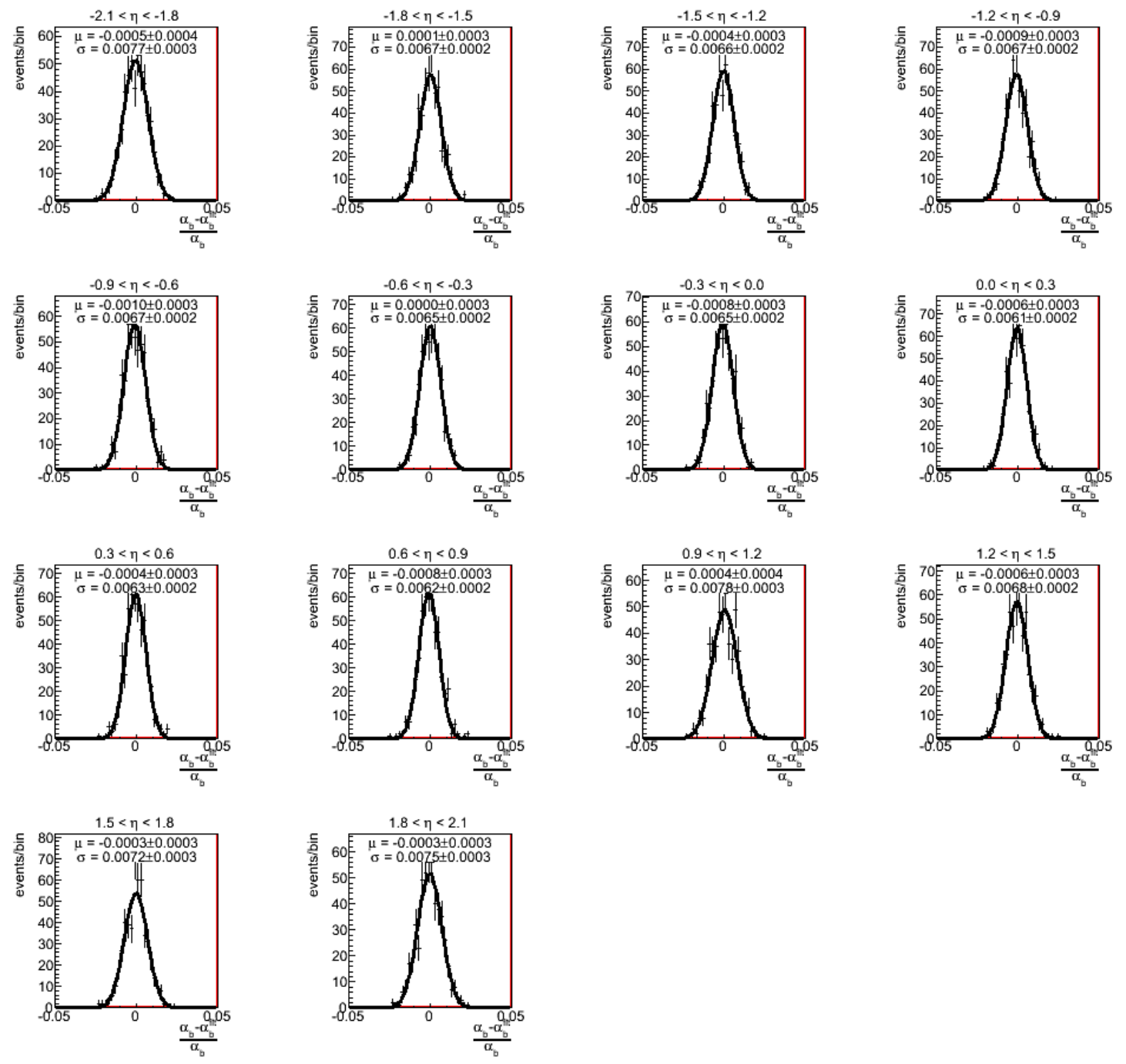

Figure A.7: Deviation of the fitted scale factor $\alpha_{b}^{f i t}$ in $b$-events from the true value $\alpha_{b}$ when using the approximate fitting method. The results are obtained from repeated pseudo experiments with data statistics generated by appropriate random variations corresponding to an integrated luminosity of $1 \mathrm{pb}^{-1}$. All bins in muon pseudorapidity are shown. 


\section{A.2.3 Pull Distributions of the Approximate Method}
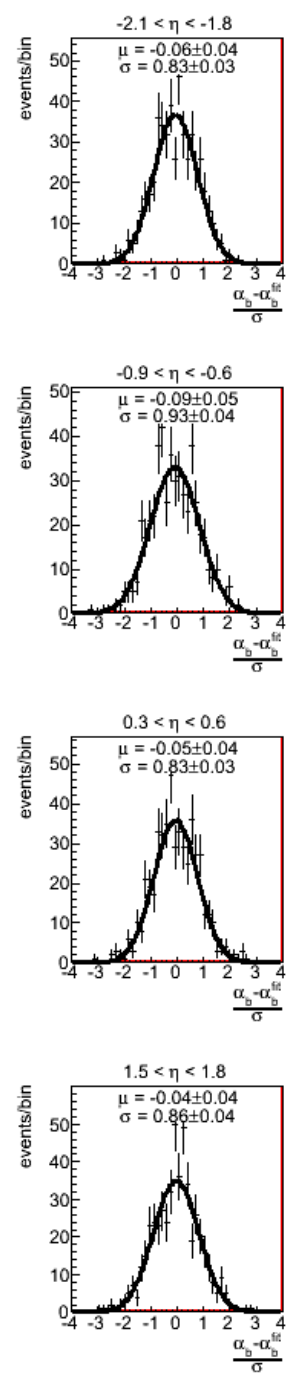
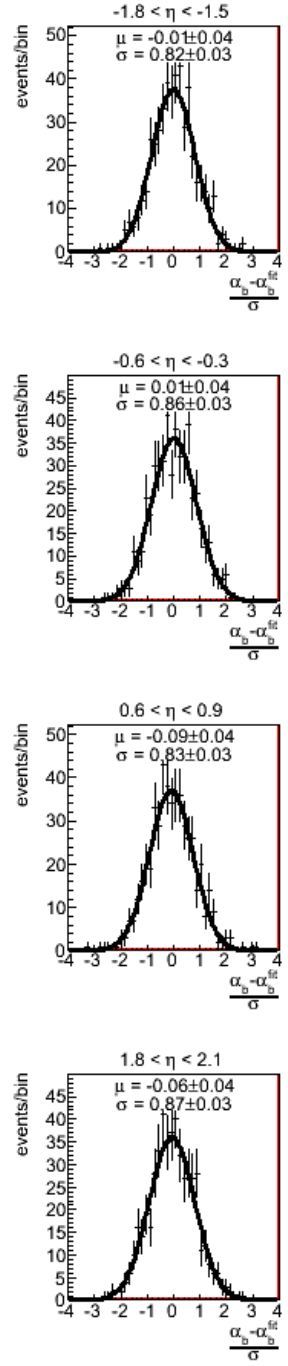
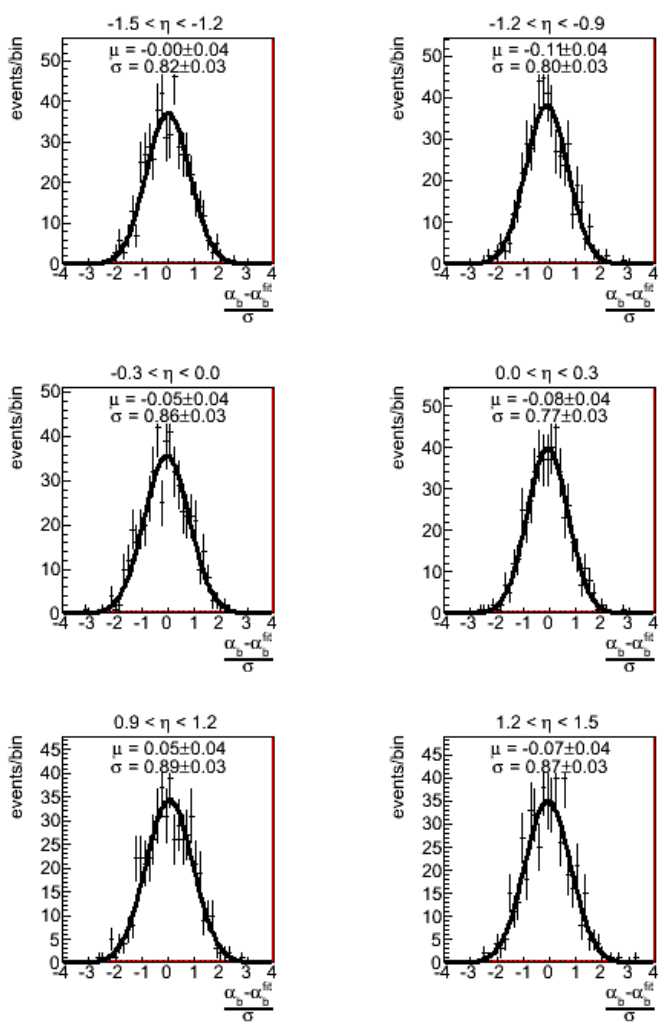

Figure A.8: $b$-fraction pull distributions resulting from the approximate fitting method. The pull distributions are obtained from repeated pseudo experiments with data statistics generated by appropriate random variations corresponding to an integrated luminosity of $1 \mathrm{pb}^{-1}$. All bins in muon pseudorapidity are shown. 


\section{A.2.4 Fit Deviation in the Full Treatment}
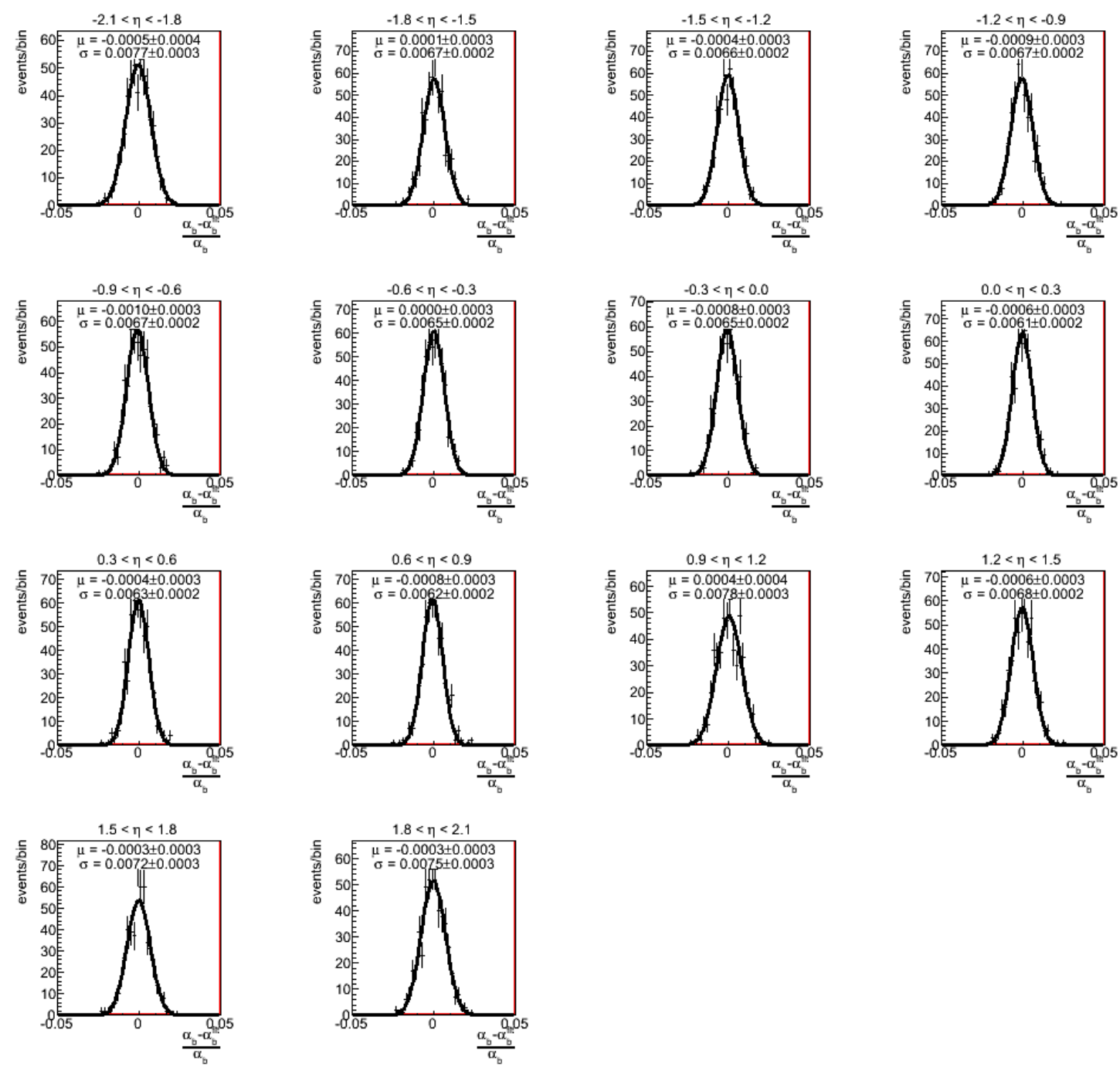

Figure A.9: Deviation of the fitted scale factor $\alpha_{b}^{f i t}$ in $b$-events from the true value $\alpha_{b}$ when using the full treatment in the fitting procedure. The results are obtained from repeated pseudo experiments with data statistics generated by appropriate random variations corresponding to an integrated luminosity of $1 \mathrm{pb}^{-1}$. All bins in muon pseudorapidity are shown. 


\section{A.2.5 Pull Distributions of the Full Treatment}
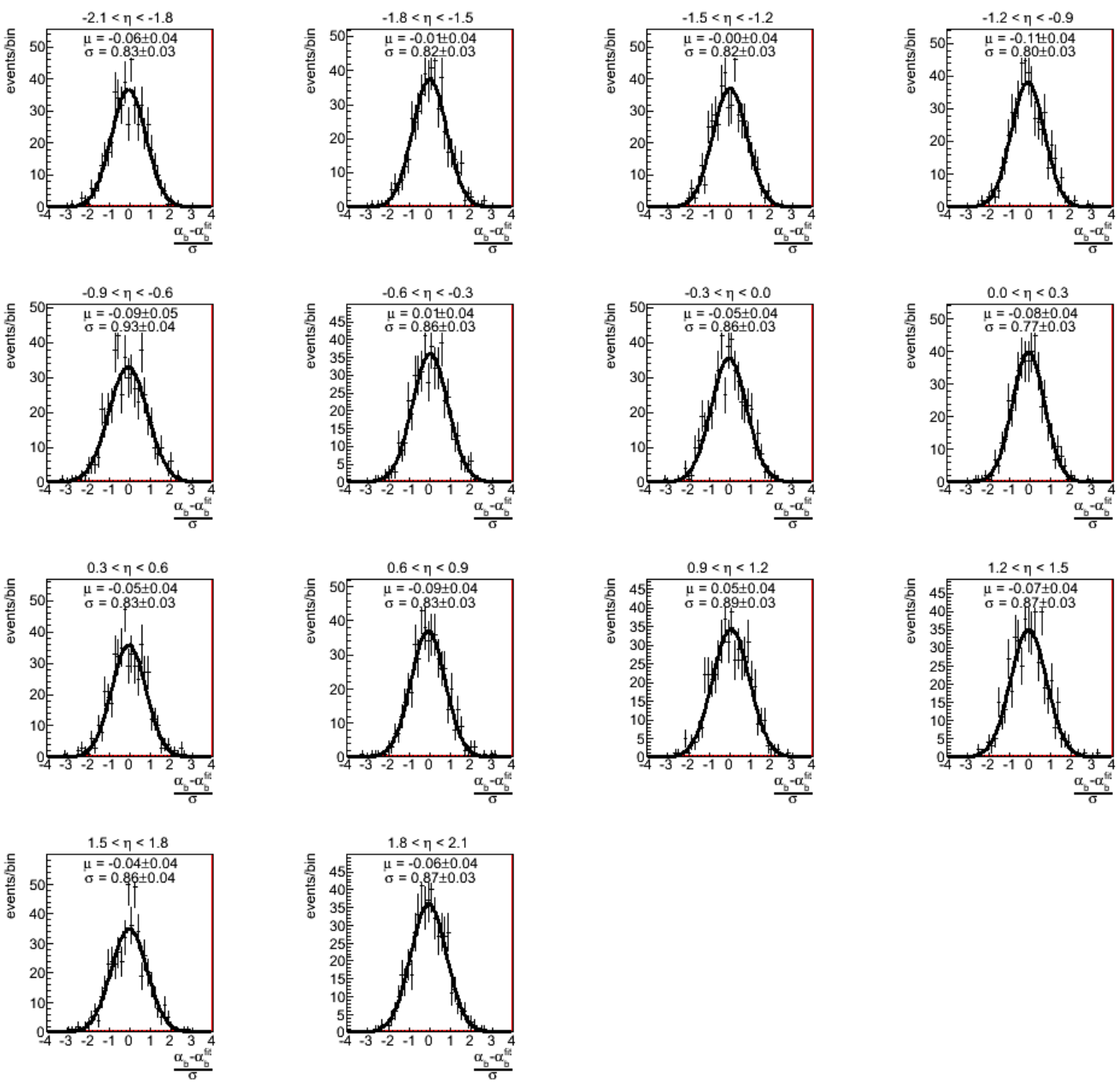

Figure A.10: $b$-fraction pull distributions resulting from the full treatment in the fitting procedure. The pull distributions are obtained from repeated pseudo experiments with data statistics generated by appropriate random variations corresponding to an integrated luminosity of $1 \mathrm{pb}^{-1}$. All bins in muon pseudorapidity are shown. 

Appendix B

Systematic Uncertainties 


\section{B.1 Limited MC Statistics}

Table B.1: Systematic uncertainties due to limited MC and data statistics.

\begin{tabular}{lc}
\hline & relative error of fitted $b$-fraction $\left(\mathcal{L}=1 \mathrm{pb}^{-1}\right)$ \\
\hline$p_{T}^{\mu}$ & \\
\hline $5-6 \mathrm{GeV}$ & $0.5 \%$ \\
$6-7 \mathrm{GeV}$ & $0.5 \%$ \\
$7-8 \mathrm{GeV}$ & $0.5 \%$ \\
$8-10 \mathrm{GeV}$ & $0.5 \%$ \\
$10-12 \mathrm{GeV}$ & $0.6 \%$ \\
$12-14 \mathrm{GeV}$ & $0.6 \%$ \\
$14-16 \mathrm{GeV}$ & $1 \%$ \\
$16-20 \mathrm{GeV}$ & $1 \%$ \\
$20-30 \mathrm{GeV}$ & $1.2 \%$ \\
$30-50 \mathrm{GeV}$ & $3 \%$ \\
\hline$\eta^{\mu}$ & \\
\hline$(-2,-1.8)$ & $0.8 \%$ \\
$(-1.8,-1.5)$ & $0.7 \%$ \\
$(-1.5,-1.2)$ & $0.7 \%$ \\
$(-1.2,-0.9)$ & $0.7 \%$ \\
$(-0.9,-0.6)$ & $0.7 \%$ \\
$(-0.6,-0.3)$ & $0.7 \%$ \\
$(-0.3,0)$ & $0.7 \%$ \\
$(0,0.3)$ & $0.7 \%$ \\
$(0.3,0.6)$ & $0.7 \%$ \\
$(0.6,0.9)$ & $0.7 \%$ \\
$(0.9,1.2)$ & $0.7 \%$ \\
$(1.2,1.5)$ & $0.7 \%$ \\
$(1.5,1.8)$ & $0.7 \%$ \\
$(1.8,2.1)$ & $0.8 \%$ \\
\hline
\end{tabular}


Appendix C

Preliminary Results of First

Collisions at $\sqrt{s}=7 \mathrm{TeV}$ 


\section{C.1 $p_{\perp}^{\text {rel }}$ Distribution in Bins of Muon Transverse Momentum}
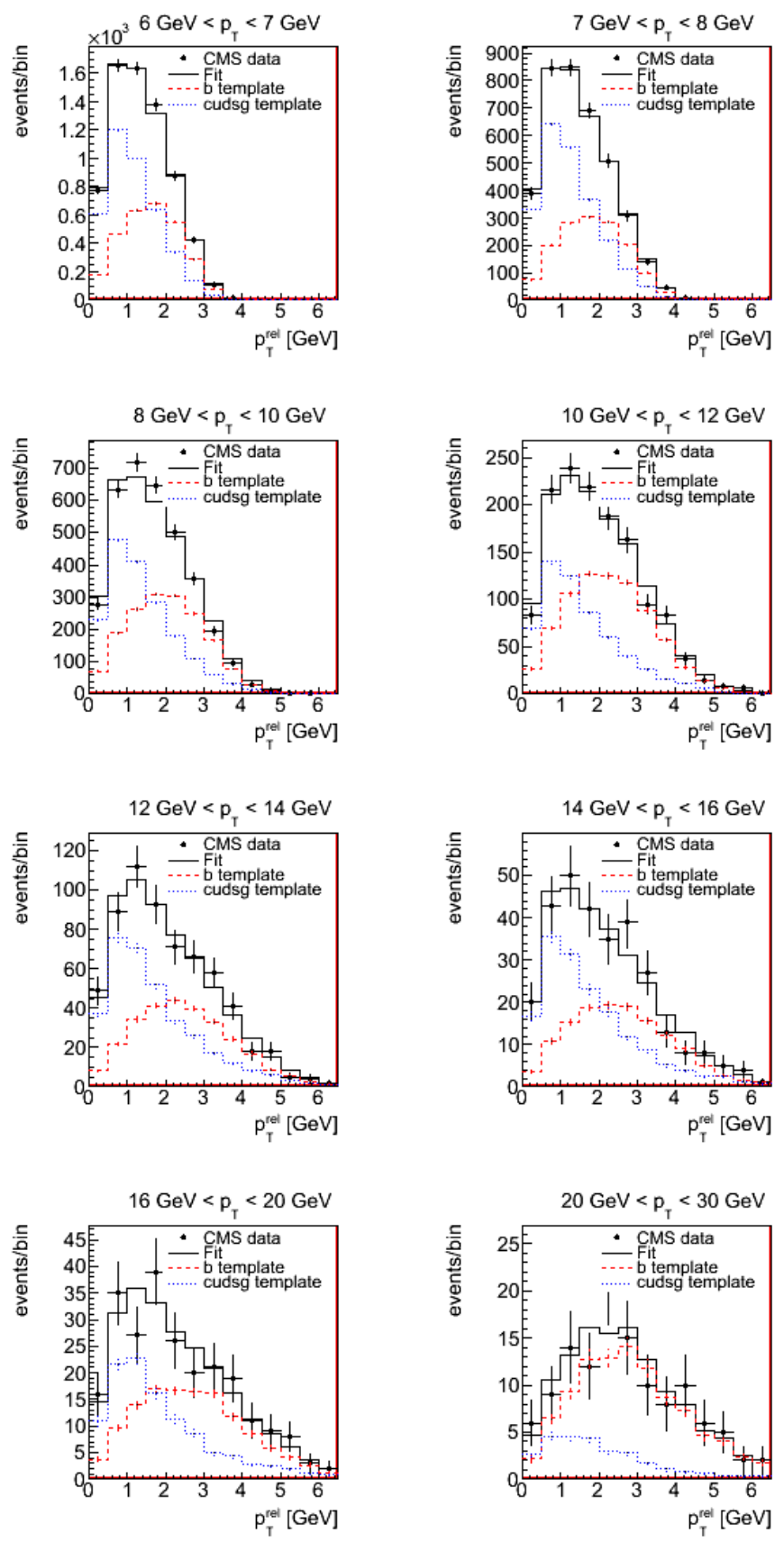

Figure C.1: $p_{\perp}^{\mathrm{rel}}$ distribution measured in data. 8 bins in muon transverse momentum are shown. The result of the maximum likelihood fit and the simulated template distributions are overlaid on the $p_{\perp}^{\mathrm{rel}}$ distribution in data. The red dashed and the blue dotted line are the $b$ - and cudsg-template, respectively. The black full circles correspond to the data distribution, while the black line is the result of the fitting procedure. 


\section{C.2 $p_{\perp}^{\mathrm{rel}}$ Distribution in Bins of Muon Pseudorapidity}
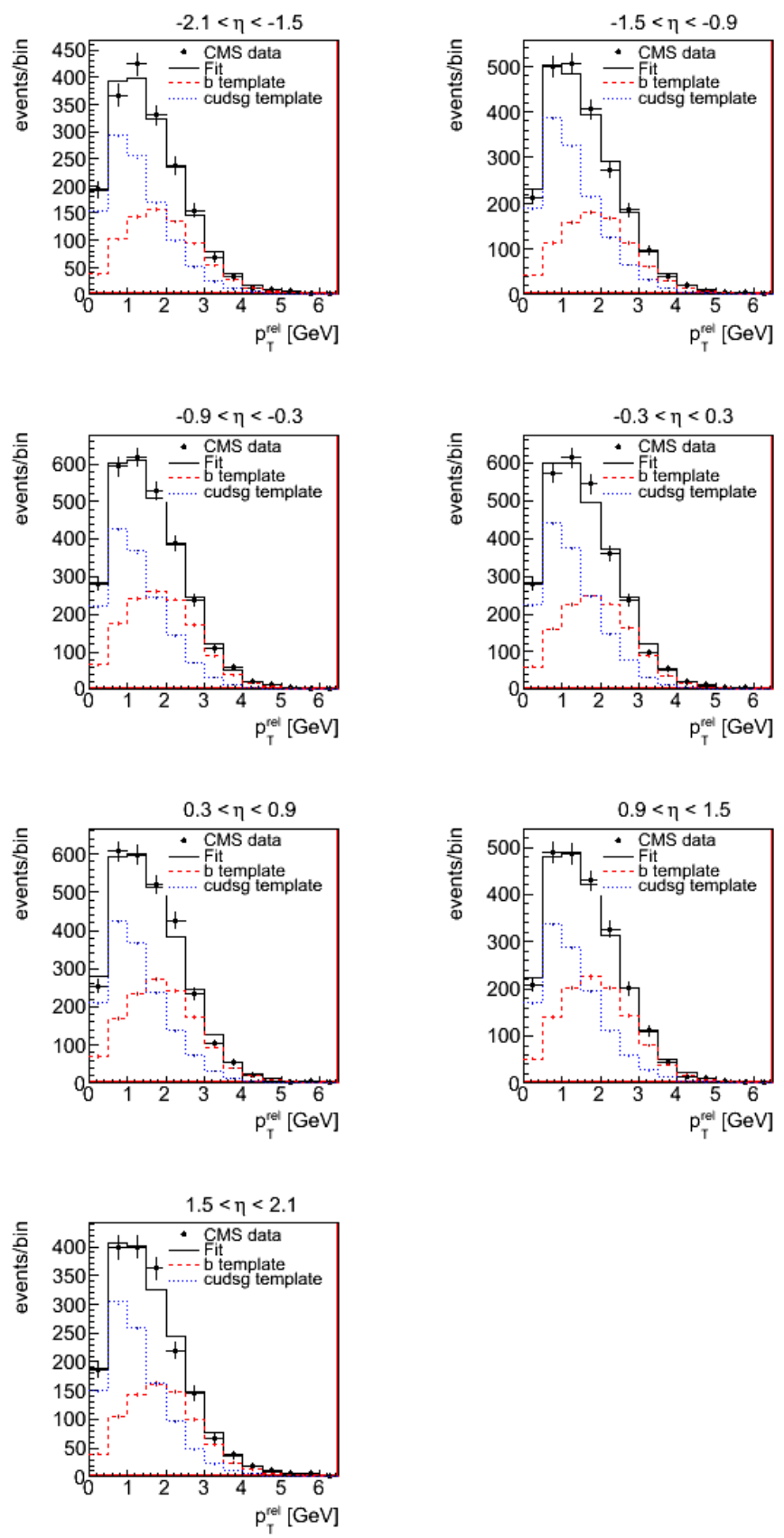

Figure C.2: $p_{\perp}^{\text {rel }}$ distribution measured in data. 7 bins in muon pseudorapidity are shown. The result of the maximum likelihood fit and the simulated template distributions are overlaid on the $p_{\perp}^{\text {rel }}$ distribution in data. The red dashed and the blue dotted line are the $b$ - and cudsg-template, respectively. The black full circles correspond to the data distribution, while the black line is the result of the fitting procedure. 



\section{List of Figures}

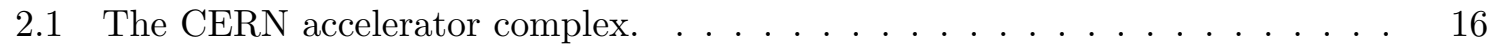

2.2 Schematic view of the CMS detector. . . . . . . . . . . . . . 17

$2.3 r z$-view of the CMS tracking detectors. . . . . . . . . . . . . . . . 19

2.4 Track reconstruction efficiency. . . . . . . . . . . . . . . . . 20

2.5 Material budget of the CMS tracker. . . . . . . . . . . . . . . . . . . . . 21

2.6 Resolution of track parameters. . . . . . . . . . . . . . . . 21

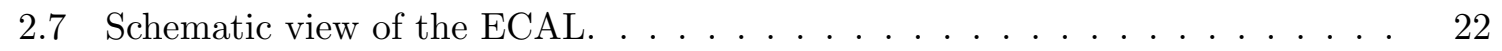

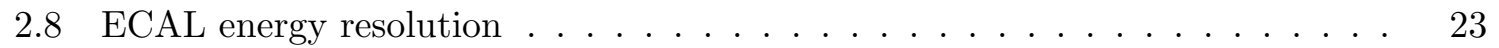

2.9 HCAL tower segmentation. . . . . . . . . . . . . . . 24

2.10 CMS muon system . . . . . . . . . . . . . . . . . 25

2.11 Muon transverse momentum resolution . . . . . . . . . . . . . . 27

2.12 L1 trigger architecture . . . . . . . . . . . . . . . . 28

3.1 Feynman graphs contributing to the $\beta$ function in the one loop approximation. 32

3.2 Summary of measurements of $\alpha_{s} \ldots \ldots \ldots \ldots \ldots \ldots \ldots$

3.3 Scattering process of two hadrons. . . . . . . . . . . . . . . . 34

3.4 Feynman diagrams contributing to the LO splitting functions. . . . . . . . . . 36

3.5 Quark and gluon distribution functions measured at HERA. . . . . . . . . . . 37

3.6 LO Feynman diagrams for heavy-quark pair production. . . . . . . . . . . . 37

3.7 NLO Feynman diagrams for heavy-quark pair production. . . . . . . . . . . 38

3.8 Total $b$ cross-section in pp collisions as a function of the center-of-mass energy. $\quad 38$

3.9 Illustration of the transverse impact parameter. . . . . . . . . . . . . . 41

3.10 Feynman graph of the semileptonic decay of $b$-hadrons. . . . . . . . . . . . 41

3.11 Schematic view of the subsequent steps of a MC event generator. . . . . . . . 43

4.1 Illustration of the $p_{\perp}^{\mathrm{rel}}$ variable. . . . . . . . . . . . . . 46

$4.2 \quad b$ quark transverse momentum and pseudorapidity distribution. . . . . . . . . . 47

4.3 Generated transverse momentum and pseudorapidity distribution of muons produced in the semileptonic decay of $b$ quarks. . . . . . . . . . . . . . . 47

4.4 Single muon trigger efficiency . . . . . . . . . . . . . . . . . 50

4.5 Jet angular resolution. . . . . . . . . . . . . . . . . . . . . . 51

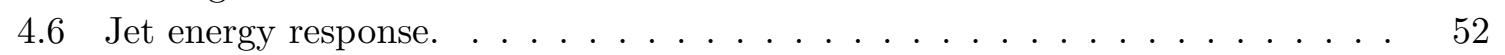

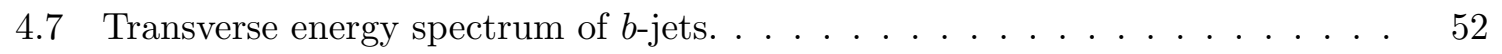

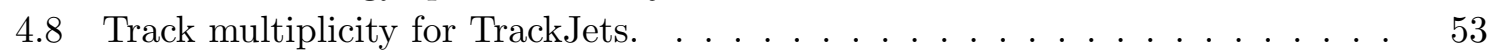

4.9 Jet finding efficiency and fake rate . . . . . . . . . . . . . 54

4.10 Simulated distribution of muon transverse momentum and pseudorapidity. . . 55 
$4.11 \Delta R$ between the reconstructed muon and the TrackJet in simulation. . . . . 55

4.12 Fraction of $b$-events in the inclusive sample obtained from simulation. . . . . 56

$4.13 p_{\perp}^{\text {rel }}$ distribution in simulated events separated by the quark content. . . . . . 56

4.14 Fit result obtained by dividing the MC sample in two independent subsamples. 60

4.15 Deviation of the fitted $b$-fraction from the $\mathrm{MC}$ value. . . . . . . . . . . . 61

4.16 Signed transverse impact parameter significance of the muon. . . . . . . . . . 62

4.17 Simulated muon fake probability for kaons, pions and baryons. . . . . . . . 63

4.18 Fitted invariant mass distribution of $D^{0}$ candidates. . . . . . . . . . . . 64

4.19 Minimum bias track spectrum scaled by the fake muon probability. . . . . . 65

4.20 Purity of the binning. . . . . . . . . . . . . . . 66

4.21 Efficiency of the online and offline event selection in simulation. . . . . . . . . 67

4.22 Jet finding efficiency as a function of the tracking inefficiency and $p_{\perp}^{\text {rel }}$ distribution for different tracking efficiencies. . . . . . . . . . . . . . . 69

4.23 Fitted $b$-fraction when varying the $c$-fraction in the background template. . . 70

4.24 Muon transverse momentum, TrackJet transverse energy and $p_{\perp}^{\text {rel }}$ distribution in FEX, FCR and GS events. . . . . . . . . . . . . . . . . 71

4.25 Differential $b$-quark production cross-section as a function of the muon transverse momentum. . . . . . . . . . . . . . . . . .

4.26 Differential $b$-quark production cross-section as a function of the muon pseudorapidity. . . . . . . . . . . . . . . .

5.1 Muon kinematic distributions in data compared to simulation at $\sqrt{s}=900 \mathrm{GeV}$

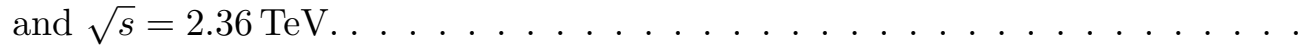

5.2 Track kinematic distributions in data compared to simulation at $\sqrt{s}=900 \mathrm{GeV}$

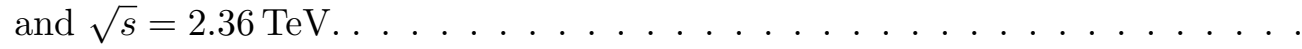

5.3 TrackJet kinematic distributions in data compared to simulation at $\sqrt{s}=$ $900 \mathrm{GeV}$ and $\sqrt{s}=2.36 \mathrm{TeV} \ldots \ldots \ldots \ldots \ldots$

5.4 TrackJet track multiplicity and transverse momentum of the highest transverse momentum track in the TrackJets in data compared to simulation. . . . . . .

5.5 Transverse impact parameter significance of tracks in TrackJets. . . . . . . . .

$5.6 p_{\perp}^{\text {rel }}$ distribution in the collision data at $\sqrt{s}=900 \mathrm{GeV}$ compared to the MC

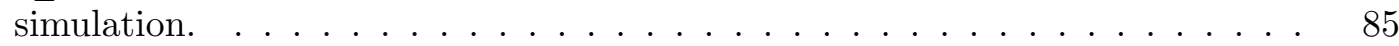

5.7 Muon fake probability as obtained from simulation. . . . . . . . . 85

$5.8 p_{\perp}^{\text {rel }}$ distribution obtained by re-weighting the hadronic track spectrum in data by the simulated muon fake probability. . . . . . . . . . . . . .

6.1 Integrated luminosity recorded by CMS during the first months of data taking

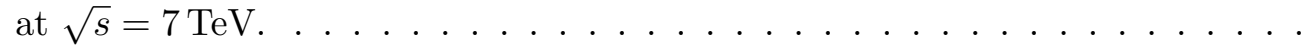

6.2 Single muon trigger efficiency (HLT_Mu3) as measured from data compared to simulation. . . . . . . . . . . . . . . . . . .

6.3 Transverse momentum, pseudorapidity, azimuthal angle and transverse impact parameter distribution of selected reconstructed global muons at $\sqrt{s}=7 \mathrm{TeV}$.

6.4 Transverse energy, pseudorapidity, azimuthal angle and number of tracks of selected TrackJets at $\sqrt{s}=7 \mathrm{TeV} \ldots \ldots \ldots \ldots$

$6.5 p_{\perp}^{\text {rel }}$ distribution in light quark background events obtained from data and sim-

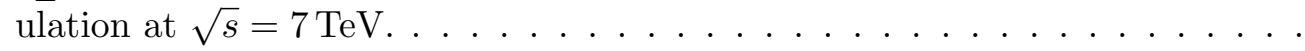


6.6 $p_{\perp}^{\mathrm{rel}}$ distribution in $b$-enriched events in data and MC obtained by a cut on the muon signed transverse impact parameter significance. . . . . . . . . . . . . 94

$6.7 p_{\perp}^{\mathrm{rel}}$ distribution measured in data at $\sqrt{s}=7 \mathrm{TeV} \ldots \ldots \ldots \ldots$

6.8 Measured differential $b$-quark production cross-section $d \sigma / d p_{T}$ as a function of the muon transverse momentum. . . . . . . . . . . . . . . .

6.9 Measured differential $b$-quark production cross-section $d \sigma / d \eta$ as a function of the muon pseudorapidity. . . . . . . . . . . . . . 98

7.1 Mechanical design of the CMS BPIX detector . . . . . . . . . . . . . 106

7.2 The CMS BPIX Module. . . . . . . . . . . . . . . . . . . 107

7.3 The sensor of the BPIX module. . . . . . . . . . . . . . . . . . 108

7.4 The BPIX Readout Chip. . . . . . . . . . . . . . . . . . . . . . . 109

7.5 Schematics of a readout chain consisting of a TBM and a group of ROCs. . . 109

7.6 Overview of the BPIX readout and control system. . . . . . . . . . . . . . 110

7.7 Analog readout of a module. . . . . . . . . . . . . . . . . . . . 111

7.8 Schematic view of one of the four BPIX supply tubes. . . . . . . . . . . . 113

7.9 Layout of the BPIX CCU board. . . . . . . . . . . . . . . . . 113

8.1 BPIX test software architecture . . . . . . . . . . . . . . . 117

8.2 Baseline, slope and noise of an $\mathrm{AOH}$ fiber as function of laser bias. . . . . . . 118

8.3 Combined coding of the LHC clock and L1 trigger signals. . . . . . . . . . . . 119

8.4 Illustration of the BPIX digital circuit. . . . . . . . . . . . . . . . . 119

8.5 Digital readback of modules in a readout group. . . . . . . . . . . . . 120

8.6 Address-levels of all pixels in a ROC. . . . . . . . . . . . . . . . . . . . 122

8.7 S-curve measurement for one pixel. . . . . . . . . . . . . . . . . 123

8.8 Measured pulse height as a function of the amplitude of the injected signal. . 123

8.9 Illustration of module mounting procedure. . . . . . . . . . . . . 125

8.10 One half of the fully assembled BPIX detector. . . . . . . . . . . . 126

8.11 The BPIX supply tube during assembly. . . . . . . . . . . . . . . . . . 127

8.12 Picture of an AOH where the two outer lasers are disconnected. . . . . . . . . 128

8.13 One half of the BPIX detector with supply tubes connected. . . . . . . . . . 128

8.14 Insertion of the BPIX detector into CMS. . . . . . . . . . . . . . 130

8.15 View of the BPIX detector in its final position. . . . . . . . . . . . . . 131

8.16 OTDR measurement of an analog optical fiber. . . . . . . . . . . . . 132

8.17 Microscope picture of the optical connection at the FED. . . . . . . . . . . 132

8.18 Result of the address level calibration . . . . . . . . . . . . . . . 134

8.19 Distribution of gain and pedestals for all pixels. . . . . . . . . . . . 135

8.20 CMS event display showing a cosmic muon traversing the pixel detector. . . . 136

8.21 Number of hits in the BPIX associated to a reconstructed track. . . . . . . . 136

A.1 Fit result obtained by using the approximate fitting method in bins of muon transverse momentum. . . . . . . . . . . . . . . . . . . . 140

A.2 Deviation of the fitted scale factor $\alpha_{b}^{f i t}$ from the true value $\alpha_{b}$ when using the approximate fitting method in bins of muon transverse momentum. . . . . . . 141

A.3 $b$-fraction pull distributions resulting from the approximate fitting method in bins of muon transverse momentum. . . . . . . . . . . . . . . . 142 
A.4 Deviation of the fitted scale factor $\alpha_{b}^{f i t}$ from the true value $\alpha_{b}$ when using the full treatment in the fitting procedure in bins of muon transverse momentum.

A.5 $b$-fraction pull distributions resulting from the full treatment in the fitting procedure in bins of muon transverse momentum. . . . . . . . . . . . . . . . . 144

A.6 Fit result obtained using the approximate fitting method in bins of muon pseudorapidity. . . . . . . . . . . . . . . . . . . . . 145

A.7 Deviation of the fitted scale factor $\alpha_{b}^{\text {fit }}$ from the true value $\alpha_{b}$ when using the approximate fitting method in bins of muon pseudorapidity. . . . . . . . . . 146

A.8 $b$-fraction pull distributions resulting from the approximate fitting method in bins of muon pseudorapidity. . . . . . . . . . . . . . . . . . 147

A.9 Deviation of the fitted scale factor $\alpha_{b}^{\text {fit }}$ from the true value $\alpha_{b}$ when using the full treatment in the fitting procedure in bins of muon pseudorapidity. . . . . 148

A.10 $b$-fraction pull distributions resulting from the full treatment in the fitting procedure in bins of muon pseudorapidity. . . . . . . . . . . . . . . . .

C.1 $p_{\perp}^{\text {rel }}$ distribution measured in data at $\sqrt{s}=7 \mathrm{TeV}$ in 8 bins in muon transverse momentum. . . . . . . . . . . . . . . . . . . . 154

C.2 $p_{\perp}^{\text {rel }}$ distribution measured in data at $\sqrt{s}=7 \mathrm{TeV}$ in 7 bins in muon pseudorapidity . . . . . . . . . . . . . . . . . 155 


\section{List of Tables}

2.1 HCAL tower segmentation. . . . . . . . . . . . . . . . 24

3.1 Peterson parameter for charm and beauty quarks. . . . . . . . . . . . 39

3.2 Properties of $b$-hadrons. . . . . . . . . . . . . . . . . 40

4.1 Event samples used in the analysis. . . . . . . . . . . . . . . . . 48

4.2 Transverse momentum threshold of the single muon trigger. . . . . . . . . . 49

4.3 Expected HLT muon transverse momentum threshold for different luminosities. 49

4.4 Selection criteria for the tracks used in the TrackJet clustering algorithm. . . 53

4.5 PYTHIA and HERWIG prediction for the relative contributions of the three production mechanisms. . . . . . . . . . . . . . . . . . . 72

4.6 Summary of systematic uncertainties . . . . . . . . . . . . . . . 73

4.7 Differential $b$-quark cross-section $d \sigma / d p_{T}$ in $\mathrm{MC}$ at $\sqrt{s}=7 \mathrm{TeV} \ldots \ldots \ldots . . . \quad 74$

4.8 Differential $b$-quark cross-section $d \sigma / d \eta$ in $\mathrm{MC}$ at $\sqrt{s}=7 \mathrm{TeV} . \ldots \ldots \ldots$

5.1 Number of data events passing the selection at $\sqrt{s}=900 \mathrm{GeV}$ and $\sqrt{s}=2.36 \mathrm{TeV} .78$

5.2 Overview of the event simulation at $\sqrt{s}=900 \mathrm{GeV}$ and $\sqrt{s}=2.36 \mathrm{TeV} . \quad \ldots \quad 78$

6.1 Overview of the event simulation at $\sqrt{s}=7 \mathrm{TeV} \ldots \ldots \ldots \ldots$

6.2 Selection criteria for the reconstructed global muons in the offline analysis. . 90

$6.3 b$-fraction determined by the $p_{\perp}^{\text {rel }}$ fit. . . . . . . . . . . . . . 95

6.4 Differential $b$-quark cross-section $d \sigma / d p_{T}$ at $\sqrt{s}=7 \mathrm{TeV} \ldots \ldots \ldots \ldots$

6.5 Differential $b$-quark cross-section $d \sigma / d \eta$ at $\sqrt{s}=7 \mathrm{TeV} \ldots \ldots \ldots \ldots$

B.1 Systematic uncertainties due to limited MC and data statistics. . . . . . . . 152 



\section{Bibliography}

[1] S. Glashow, Partial symmetries of weak interactions, Nucl. Phys., 22 (1961) 579588.

[2] S. Weinberg, A model of leptons, Phys. Rev. Lett., 19 (1967) 12641266.

[3] A. Salam, Elementary Particle Physics: Relativistic Groups and Analyticity (Nobel Symposium No. 8) (ed. Svartholm, N.) 367 (Almqvist and Wiksills, Stockholm, 1968).

[4] UA1 Collaboration, Beauty production at the CERN proton-antiproton collider, Phys. Lett., B186 (1987) 237.

[5] ALEPH Collaboration, Nucl. Instrum. Meth., A346 (1994) 461.

[6] L3 Collaboration, Phys. Rev. Lett., B252 (1990) 703.

[7] U. Langenegger, A Measurement of the Beauty and Charm Production Cross Sections at the ep collider HERA, PhD thesis, ETH Zürich (1998) ETH No. 12676.

[8] DØ Collaboration, Inclusive $\mu$ and b-Quark Production Cross Sections in $p \bar{p}$ Collisions at $\sqrt{s}=1.8 \mathrm{TeV}$, Phys. Rev. Lett., 74 (1995) 3548.

[9] CDF Collaboration, Measurement of the bottom quark production cross section using semileptonic decay electrons in $p \bar{p}$ collisions at $\sqrt{s}=1.8$ TeV, Phys. Rev. Lett., 71 (1993) 500504 .

[10] M. L. Mangano, The saga of bottom production in $p \bar{p}$ collisions, AIP Conf. Proc. 753 (2005) 247-260.

[11] O. E. Bruning et al., LHC design report. Vol. I: The LHC main ring, CERN-2004-003-V-1 (2004).

[12] ATLAS Collaboration, Detector and physics performance technical design report. Volume 1, CERN-LHCC-99-14 (1999).

[13] CMS Collaboration, The Compact Muon Solenoid: Technical proposal, CERN-LHCC94-38 (1994).

[14] CMS Collaboration, The CMS experiment at the CERN LHC, JINST 3 S08004 (2008).

[15] LHCb Collaboration, LHCb technical proposal, CERN-LHCC-98-04 (1998).

[16] ALICE Collaboration, Technical proposal for a large ion collider experiment at the CERN LHC, CERN-LHCC-95-71 (1995). 
[17] W. Adam et al., Track reconstruction in the CMS tracker, CMS NOTE-2006/041 (2006).

[18] P. Billoir, Comput. Phys. Commun., 57 (1989) 390.

[19] CMS Collaboration, CMS High Level Trigger, CERN/LHCC 2007-021 (2007).

[20] D. J. Gross and F. Wilczek, Asymptotically Free Gauge Theories. 1, Phys. Rev., D8 (1973) 3633-3652.

[21] H. D. Politzer, Reliable Perturbative Results for Strong Interactions, Phys. Rev. Lett., 30 (1973) 1346-1349.

[22] D. J. Gross and F. Wilczek, Ultraviolet Behavior of Bon-Abelian Gauge Theories, Phys. Rev. Lett., 30 (1973) 1343-1346.

[23] H. D. Politzer, Asymptotic Freedom: An Approach to Strong Interactions, Phys. Rept., 14 (1974) 129-180.

[24] R. P. Feynman, Quantum Electrodynamics, W. A. Benjamin, Inc., New York (1961).

[25] S. S. Schweber, QED and the men who made it: Dyson, Feynman, Schwinger, and Tomonaga, Princeton, USA: Univ. Pr. (1994) 732 p.

[26] R. K. Ellis, W. J. Stirling and B. R. Webber, QCD and Collider Physics, Cambridge Monographs (2003).

[27] G. Dissertori, I. G. Knowles, M. Schmelling, Quantum Chromodynamics: High Energy Experiments and Theory, Oxford Science Publications (2003).

[28] G. Sterman et al., Handbook of perturbative QCD, Rev. Mod. Phys., 67 (1995) 157-248.

[29] S. Bethke, The 2009 World Average of $\alpha_{s}$, Eur. Phys. J., C64 (2009) 689-703.

[30] R. K. Ellis, H. Georgi, M. Machacek, H. D. Politzer and G. G. Ross, Perturbation Theory and the Parton Model in QCD, Nucl. Phys., B152 (1979) 285.

[31] J. C. Collins, D. E. Soper and G. Sterman, Factorization of Hard Processes in QCD, Adv. Ser. Direct. High Energy Phys., 5 (1988) 1-91.

[32] J. C. Collins and D. E. Soper, The Theorems of Perturbative QCD, Ann. Rev. Nucl. Part. Sci., 37 (1987) 383-409.

[33] J. C. Collins, D. E. Soper and G. Sterman, Nucl. Phys., B263 (1986) 37.

[34] V. N. Gribov and L. N. Lipatov, $e^{+} e^{-}$pair annihilation and deep inelastic ep scattering in perturbation theory, Sov. J. Nucl. Phys., 15 (1972) 675-684.

[35] V. N. Gribov and L. N. Lipatov, Deep inelastic e p scattering in perturbation theory, Sov. J. Nucl. Phys., 15 (1972) 438-450.

[36] G. Altarelli and G. Parisi, Asymptotic Freedom in Parton Language, Nucl. Phys., B126 (1977) 298. 
[37] Y. L. Dokshitzer, Calculation of the Structure Functions for Deep Inelastic Scattering and $e^{+} e^{-}$Annihilation by Perturbation Theory in Quantum Chromodynamics, Sov. Phys. JETP 46 (1977) 641-653.

[38] J. Pumplin, D. R. Stump, J. Huston, H. L. Lai, P. M. Nadolsky and W. K. Tung, New generation of parton distributions with uncertainties from global QCD analysis, JHEP 0207 (2002) 012.

[39] A. D. Martin, R. G. Roberts, W. J. Stirling and R. S. Thorne, MRST2001: Partons and $\alpha_{s}$ from precise deep inelastic scattering and Tevatron jet data, Eur. Phys. J., C23 (2002) 73.

[40] A. D. Martin, W. J. Stirling, R. S. Thorne, G. Watt, Parton distributions for the LHC, Eur. Phys. J., C63 (2009) 189-285.

[41] R. D. Ball et al. [NNPDF Collaboration], A determination of parton distributions with faithful uncertainty estimation, Nucl. Phys., B809 (2009) 1 [Erratum-ibid., B816 (2009) 293].

[42] H1 and ZEUS Collaborations, Combined Measurement and QCD Analysis of the Inclusive ep Scattering Cross Sections at HERA, JHEP 1001 (2010) 109.

[43] M. L. Mangano, Two Lectures on Heavy Quark Production in Hadronic Collisions, CERN-TH/97-328 (1997).

[44] E. Norrbin and T. Sjostrand, Production and hadronization of heavy quarks, Eur. Phys. J., C17 (2000) 137.

[45] P. Nason and C. Oleari, Nucl. Phys., B565 (2000) 245.

[46] C. Peterson, D. Schlatter, I. Schmitt and Peter M. Zerwas, Scaling Violations in Inclusive $e^{+} e^{-}$Annihilation Spectra, Phys. Rev., D27 (1983) 105.

[47] N. Cabibbo, Unitarity Symmetry and Leptonic Decays, Phys. Rev. Lett. 10 (1963) 531533.

[48] M. Kobayashi and T. Maskawa, CP-Violation in the Renormalizable Theory of Weak Interaction, Prog. Theor. Phys. 49 (1973) 652-657.

[49] C. Amsler et al. (Particle Data Group), Phys. Lett., B667 (2008) 1.

[50] H.C. Kästli et al., CMS barrel pixel detector overview, Nucl. Instrum. Meth., A582 (2007) 724 .

[51] R. Weber, Diffractive $\rho^{0}$ photoproduction at HERA, PhD thesis, ETH Zürich (2006) ETH No. 16709.

[52] H. U. Bengtsson and T. Sjöstrand, Comp. Phys. Comm., 46 (1987) 367.

[53] T. Sjöstrand, Comp. Phys. Comm., 82 (1994) 74.

[54] T. Sjöstrand, S. Mrenna, and P. Z. Skands, PYTHIA 6.4 Physics and Manual, JHEP 05 (2006) 026. 
[55] G. Marchesini and B. R. Webber, Nucl. Phys., B310 (1988) 461.

[56] G. Marchesini, B. R. Webber, G. Abbiendi, I. G. Knowles, M. H. Seymour and L. Stanco, Comp. Phys. Comm., 67 (1992) 465.

[57] G. Corcella et al., HERWIG 6.5: an event generator for Hadron Emission Reactions With Interfering Gluons (including supersymmetric processes), JHEP 01 (2001) 010.

[58] S. Frixione and B. R. Webber, Matching NLO QCD computations and parton shower simulations, JHEP 0206 (2002) 029.

[59] S. Frixione, P. Nason and B.R. Webber, Matching NLO QCD and parton showers in heavy flavour production, JHEP 0308 (2003) 007.

[60] ATLAS, CMS, and TOTEM Collaboration, Multiple parton interactions, underlying event and forward physics at LHC, Proceedings of Multiple Parton Interactions at the LHC, $1^{\text {st }}$ Workshop, DESY-PROC-2009-06 (2008).

[61] T. Sjöstrand, Comp. Phys. Comm., 39 (1986) 347.

[62] M. Bengtsson and T. Sjöstrand, Comp. Phys. Comm., 43 (1987) 367.

[63] B. Andersson, G. Gustafson and B. Söderberg, Z. Phys., C20 (1983) 317.

[64] http://www.slac.stanford.edu/ lange/EvtGen/

[65] J. M. Butterworth, J. R. Forshaw and M. H. Seymour, Multiparton interactions in photoproduction at HERA, Z. Phys. C72 (1996) 637646.

[66] DØ Collaboration, The $b \bar{b}$ Production Cross Section and Angular Correlations in $p \bar{p}$ Collisions at $\sqrt{s}=1.8$ TeV, Phys. Lett., B487 (2000) 264.

[67] CDF Collaboration, Measurement of the b-Hadron Production Cross Section Using Decays to $\mu^{-} D^{0} X$ Final States in $p \bar{p}$ Collisions at $\sqrt{s}=1.96$ TeV, Phys. Rev., D79 (2009) 092003.

[68] H1 Collaboration, Measurement of Beauty Production at HERA Using Events with Muons and Jets, Eur. Phys. J., C41 (2005) 453-467.

[69] H1 Collaboration, Measurement of Charm and Beauty Photoproduction at HERA using $D^{*} \mu$ Correlations, Phys. Lett., B621 (2005) 56-71.

[70] ZEUS Collaboration, Measurement of Beauty Photoproduction using Decays into Muons in Dijet Events at HERA, JHEP 0904 (2009) 133.

[71] ZEUS Collaboration, Measurement of charm and beauty production in deep inelastic ep scattering from decays into muons at HERA, Eur. Phys. J., C65 (2010) 65-79.

[72] Geant4 Collaboration, Geant4 - a simulation toolkit, Nucl. Inst. Meth., A506 (2003) 250-303.

[73] M. Cacciari, G. P. Salam and G. Soyez, The anti- $k_{T}$ jet clustering algorithm, JHEP 0804 (2008) 063. 
[74] P. Azzuri et al., Performance of Jet Reconstruction with Charged Tracks only, CMS AN-2008/041 (2008).

[75] P. Schieferdecker et al., Performance of Jet Algorithms in CMS, CMS AN-2008/001 (2008).

[76] CMS Collaboration, Plans for Jet Energy Corrections at CMS, CMS PAS JME-07-002 (2007).

[77] L. Caminada, W.Erdmann, H.-C. Kästli, Study of the inclusive b production cross-section in CMS, CMS AN-2009/160 (2009).

[78] V. Andreev et al., Measurement of open beauty production at LHC with CMS, CMS AN-2006/120 (2006).

[79] R. Barlow, C. Beeston, Comput. Phys. Commun., 77 (1993) 219.

[80] ROOT: an object oriented data analysis framework, http://root.cern.ch.

[81] http://root.cern.ch/root/htmldoc/TFractionFitter.html.

[82] http://wwwasdoc.web.cern.ch/wwwasdoc/minuit/minmain.html.

[83] G. Zech, Comparing Statistical Data to Monte Carlo Simulation - Parameter Fitting and Unfolding, DESY 95-113 (1995).

[84] CDF Collaboration, Measurement of correlated $b \bar{b}$ production in $p \bar{p}$ collisions at $\sqrt{s}=1960$ GeV, Phys. Rev. D77 (2008) 072004 .

[85] D. Bortoletto et al., A Measurement of the Inclusive Production of the $\Upsilon$ Resonance at $\sqrt{s}=10$ TeV with an Integrated Luminosity of $1 \mathrm{pb}^{-1}$, CMS AN-2009/118 (2009).

[86] CMS Collaboration, Measurement of Tracking Efficiency, CMS PAS TRK-10-002 (2010).

[87] L. Caminada, W. Erdmann, V. Zhukov, M. Niegel, D. Troendle, Measurement of the cross section for open-beauty production with muons and jets in pp Collisions at $\sqrt{s}=$ $7 \mathrm{TeV}$, CMS AN-2010/171 (2010).

[88] CMS Collaboration, Measurement of CMS Luminosity, CMS PAS EWK-10-004 (2010).

[89] G. D. Lafferty, T. R. Wyatt, Where to stick your data points: The treatment of measurements within wide bins, Nucl. Instrum. Meth., A355 (1994) 541-547.

[90] A. J. Bell, Design and Construction of the Beam Scintillation Counter for CMS, Master's thesis, University of Canterbury, New Zealand (2008).

[91] T. Aumeyr, Beam Phase and Intensity Monitoring for the Compact Muon Solenoid Experiment, Master's thesis, Vienna University of Technology, Austria (2008).

[92] CMS Collaboration, Tracking and Vertexing Results from First Collisons, CMS PAS TRK-10-001 (2010).

[93] P. Bartalini and L. Fano, Multiple Parton Interactions at the LHC, DESY-PROC-2009-06 (2009). 
[94] CMS Collaboration, Alignment of the CMS Silicon Tracker during Commissioning with Cosmic Rays, JINST, 5 (2010) T03009.

[95] CMS Collaboration, Measurement of the Underlying Event Activity in Proton-Proton Collisions at $900 \mathrm{GeV}$, CMS PAS QCD-10-001 (2010).

[96] CMS Collaboration, Open beauty production cross section with muons in pp collisions at $\sqrt{s}=7 \mathrm{Te}$, CMS PAS BPH-10-007 (2010).

[97] L. Caminada, Measurement of the inclusive $b$ production cross section in pp collisions at $\sqrt{s}=7 T e V, 35^{\text {th }}$ International Conference On High Energy Physics (ICHEP 2010).

[98] CMS Collaboration, Tracking and Primary Vertex Results in First $7 \mathrm{TeV}$ Collisions, CMS PAS TRK-10-005 (2010).

[99] M. Cacciari, S. Frixione, M. L. Mangano et al., QCD analysis of first b cross-section data at $1.96 \mathrm{TeV}$, JHEP 07 (2004) 033.

[100] Y. Allkofer et al., Design and performance of the silicon sensors for the CMS barrel pixel detector, Nucl. Instrum. Meth., A584 (2008) 25.

[101] H.C. Kästli et al., Design and performance of the CMS pixel detector readout chip, Nucl. Instrum. Meth. A565 (2006) 188.

[102] E. Bartz, The token bit manager chip for the CMS pixel readout, Proceeding of the Ninth Workshop on Electronics for LHC Experiments, CERN 2003-006, CERN/LHCC/2003-055 (2003).

[103] D. Kotlinski et al., The control and readout systems of the CMS pixel barrel detector, Nucl. Instrum. Meth., A565 (2006) 73.

[104] Analog Optohybrids, Technical Specifications, http://aoh.hephy.at.

[105] P. Trüb, CMS pixel module qualification and Monte-Carlo study of $H \rightarrow \tau^{+} \tau^{-} \rightarrow l^{+} l^{-} E_{T}$, PhD Thesis, ETH Zürich (2008) ETH No. 17985.

[106] M. Pernicka et al., The CMS Pixel FED, Proceedings of the Topical Workshop on Electronics for Particle Physics, Prague, Czech Republic, pp. 487-491 (2007).

[107] J. Troska et al., IEEE Trans. Nucl. Sci. NS-40 (4) (2003) 1067.

[108] P. Placidi et al., CMS Tracker PLL Reference Manual, CERN Document Server.

[109] H. Correia et al., Delay25 - A 4 channel 1/2 ns programmable delay line, CERN$\mathrm{EP} / \mathrm{MIC}(2000) 14$.

[110] C. Paillard et al., Proceedings of the Eighth Workshop on Electronics for LHC Experiments, CERN 2002-003, CERN/LHCC/2002-034 (2002).

[111] K.A. Gill et al., Proceedings of the Eleventh Workshop on Electronics for LHC Experiments (LECC 2005), CERN-2005-011, CERN-LHCC-2005-038 (2005).

[112] L. Caminada, A. Starodumov, Building and commissioning of the CMS pixel barrel detector, JINST 4 (2009) P03017. 
[113] S. Sechrest, An introductory 4.4.bsd interprocess communication tutorial, Computer Science Research Group, University of California, Berkeley, USA (1993).

[114] Python Programming Language, http://www.python.org.

[115] J. Gutleber and L. Orsini, Software Architecture for Processing Clusters Based on $\mathrm{I}_{2} \mathrm{O}$, Cluster Computing, 5 (2002) 55.

[116] D. Kotlinski, Status of the CMS Pixel detector, JINST 4 (2009) P03019.

[117] S. König et al., Building CMS pixel barrel detector modules, Nucl. Instrum. Meth. A582 (2007) 776.

[118] Christina Eggel, CMS Pixel Module Qualification and Search for $B_{s}^{0} \rightarrow \mu^{+} \mu^{-}, \mathrm{PhD}$ Thesis, ETH Zürich (2009) ETH No. 18232.

[119] A. Starodumov et al., Qualification procedures of the CMS pixel barrel modules, Nucl. Instrum. Meth. A565 (2006) 67.

[120] CMS Collaboration, Commissioning and Performance of the CMS Pixel Tracker with Cosmic Ray Muons, JINST 5 (2010) T03007. 



\section{Acknowledgement}

It was a great pleasure and a unique experience to work in a large international collaboration like CMS. This work would not have been possible without the help of many people and I am grateful to everybody who contributed to its successful completion. However, there are some people I would like to especially mention.

I wish to express my sincere thank to Felicitas Pauss for giving me the opportunity to do my thesis under her supervision. I very much appreciated her competent advise, her enthusiasm and her continuos support.

I am grateful to Ralph Eichler for making it possible to carry out my PhD work within the CMS groups at ETH and PSI. I am pleased that Ralph Eichler readily accepted to be a co-examiner for this thesis and I would like to thank him for his valuable comments.

Special thanks go to Roland Horisberger for introducing me to the CMS pixel community and sharing his great expertise. Working in his group was a pleasant and challenging activity. I am happy that Roland Horisberger acted as a co-examiner for this work.

I enjoyed the supervision of Christoph Grab and I would like to thank him for his advise throughout all parts of this thesis. His suggestions improved this work considerably.

I am indebted to Wolfram Erdmann for his outstanding support with regard to every aspect of my work. He was always there when a question occurred and I could profit a lot from his expert knowledge. His excellent guidance truly enriched my research work.

I would like to thank my colleagues of the Institute of Particle Physics at ETH, the CMS Pixel Group at PSI and the CMS Collaboration. I was very lucky to meet talented, inspiring and interesting people.

Finally, I would like to sincerely thank my parents, my sister and Christian for their incessant support. Their patience, their advice and encouragement are invaluable for me. 



\section{Curriculum Vitae}

\section{Personal Data}

Name

Lea Michaela Caminada

Date of Birth

December 30, 1981

Place of Birth

Nationality

Zürich (ZH)

Citizen of

Swiss

Vrin (GR) and Zürich (ZH)

\section{Education}

$2006-2010$

Doctoral studies at ETH Zürich and PSI

Research at the CMS experiment at the CERN LHC

2006

Diploma in Physics at ETH Zürich summa cum laude

Diploma Thesis at the H1 experiment at DESY in Hamburg in the group of Prof. R. Eichler

Title: Implementation of a Trigger for the Decay $b \rightarrow e+X$ on the Third Trigger Level at the H1 experiment

2003-2004

2001-2006

2001

Exchange year at the Strathclyde University in Glasgow

Studies of Physics at ETH Zürich

Eidgenössische Matura Typus B

Gymnasium in Zürich-Oerlikon

1994-2001

Primary School in Zürich-Höngg 